\title{
Strong Partial Clones and the Complexity of Constraint Satisfaction Problems
}

Limitations and Applications

\author{
Victor Lagerkvist
}

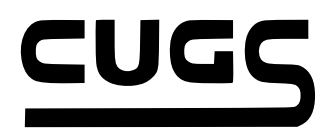

Linköping University

Department of Computer and Information Science

Division for Software and Systems

SE-581 83 Linköping, Sweden 
(C) Victor Lagerkvist, 2016

ISBN 978-91-7685-856-1

ISSN 0345-7524

URL http://urn.kb.se/resolve?urn=urn:nbn:se:liu:diva-122827/

Typeset using $\mathrm{HT}_{\mathrm{E}} \mathrm{X}$

Printed by LiU-Tryck, Linköping 2015 


\begin{abstract}
In this thesis we study the worst-case time complexity of the constraint satisfaction problem parameterized by a constraint language $(\operatorname{CSP}(S))$, which is the problem of determining whether a conjunctive formula over $S$ has a model. To study the complexity of $\operatorname{CSP}(S)$ we borrow methods from universal algebra. In particular, we consider algebras of partial functions, called strong partial clones. This algebraic approach allows us to obtain a more nuanced view of the complexity $\operatorname{CSP}(S)$ than possible with algebras of total functions, clones.

The results of this thesis is split into two main parts. In the first part we investigate properties of strong partial clones, beginning with a classification of weak bases for all Boolean relational clones. Weak bases are constraint languages where the corresponding strong partial clones in a certain sense are extraordinarily large, and they provide a rich amount of information regarding the complexity of the corresponding CSP problems. We then proceed by classifying the Boolean relational clones according to whether it is possible to represent every relation by a conjunctive, logical formula over the weak base without needing more than a polynomial number of existentially quantified variables. A relational clone satisfying this condition is called polynomially closed and we show that this property has a close relationship with the concept of few subpowers. Using this classification we prove that a strong partial clone is of infinite order if (1) the total functions in the strong partial clone are essentially unary and (2) the corresponding constraint language is finite. Despite this, we prove that these strong partial clones can be succinctly represented with finite sets of partial functions, bounded bases, by considering stronger notions of closure than functional composition.

In the second part of this thesis we apply the theory developed in the first part. We begin by studying the complexity of $\operatorname{CSP}(S)$ where $S$ is a Boolean constraint language, the generalised satisfiability problem $(\mathrm{SAT}(S))$. Using weak bases we prove that there exists a relation $R_{1 / 3}^{\neq \neq \neq}$ such that $\operatorname{SAT}\left(\left\{R_{1 / 3}^{\neq \neq \neq}\right\}\right)$is the easiest NP-complete $\operatorname{SAT}(S)$ problem. We rule out the possibility that $\operatorname{SAT}\left(\left\{R_{1 / 3}^{\neq \neq \neq}\right\}\right)$is solvable in subexponential time unless a well-known complexity theoretical conjecture, the exponential-time hypothesis, (ETH) is false. We then proceed to study the computational complexity of two optimisation variants of the SAT $(S)$ problem: the maximum ones problem over a Boolean constraint language $S$ (MAX-ONES $(S)$ ) and the valued constraint satisfaction problem over a set of Boolean cost functions $\Delta(\operatorname{VCSP}(\Delta))$. For MAX-ONES $(S)$ we use partial clone theory and prove that $\operatorname{MAX}-\mathrm{ONES}\left(\left\{R_{1 / 3}^{\neq \neq}\right\}\right)$is the easiest NPcomplete $\operatorname{MAX}-\mathrm{OnES}(S)$ problem. These algebraic techniques do not work for $\operatorname{VCSP}(\Delta)$, however, where we instead use multimorphisms to prove that MAX-CUT is the easiest NP-complete Boolean $\operatorname{VCSP}(\Delta)$ problem. Similar to the case of $\operatorname{SAT}(S)$ we then rule out the possibility of subexponential algorithms for these problems, unless the ETH is false.

This work has been supported by the Theoretical Computer Science Laboratory, Department of Computer and Information Science, Linköping University, and in part by CUGS (the National Graduate School in Computer Science, Sweden).
\end{abstract}





\section{Populärvetenskaplig sammanfattning}

I den här avhandlingen studerar vi komplexitet hos villkorsproblem. För att kunna redogöra innebörden i dessa begrepp måste vi börja på en högre nivå och förankra det forskningsområde som avhandlingen berör. Datavetenskap är ett enormt forskningsområde som innefattar i stort sett allt mellan algoritmer, programmering, realtidssystem, interaktion mellan datorer och människor, och artificiell intelligens. Teoretisk datalogi (på engelska "theoretical computer science") kan beskrivas som en delmängd av datavetenskap där vi istället för att studera konkreta datorer istället studerar modeller av hur datorer kan fungera. Alltså är de objekt vi studerar inte fysiska fenomen, utan matematiska koncept. En direkt konsekvens av detta är att teoretisk datalogi sällan innefattar studier, observationer eller experiment, som annars är centrala aspekter i de flesta vetenskaper. Därför är teoretisk datalogi närmare besläktat med matematik och logik än exempelvis fysik eller biologi.

Precis som i många andra vetenskaper arbetar vi dock med olika taxonomier och metoder för att gruppera och kategorisera olika typer av objekt och företeelser. Ett centralt koncept inom teoretisk datalogi är beräkningsproblem (på engelska "computational problem") och komplexiteten hos sådana. Beräkningsproblem kan exempelvis ha följande form.

1. Givet två tal $x$ och $y$, beräkna summan av dessa.

2. Givet två städer i en karta, beräkna den kortaste rutten mellan dem.

3. Givet en karta med $n$ städer och ett värde $k$, existerar det en rutt som besöker alla $n$ städer som inte överskrider längden $k$ ?

Alltså har vi en instans, exempelvis två nummer eller en karta, och en fråga som vi vill ha ett svar till. Det bör inte komma som någon överraskn- 
ing för läsaren att vissa beräkningsproblem i någon mening är svårare än andra. Att beräkna summan av två tal klarar till och med den enklaste miniräknaren av, medan det är betydligt svårare att hitta den kortaste rutten mellan två städer. Men hur ska man då mäta hur svårt, hur komplext, ett beräkningsproblem är? Det vanligaste, och det som vi begränsar oss till i denna avhandling, är hur mycket tid som problemet kräver för att kunna lösas. Med tid menar vi inte fysisk tid som sekunder eller minuter, utan hur många operationer som krävs i en underliggande datormodell. Detta är måhända ett inte helt intuitivt användande av begreppet "tid", men det tillåter oss att studera komplexitet hos beräkningsproblem utan att begränsa oss till fysiska resurser hos en specifik dator. En fördel med detta är att sådana analyser åldras med värdighet: till exempel så är femtio år gamla tidskomplexitetsanalyser fortfarande relevanta, vilket inte skulle vara fallet om dessa analyser istället utfördes med avseende på exekveringstiden hos en specifik dator.

Vi har nu en grundläggande förståelse för vad ett beräkningsproblem är och vad som avses med komplexitet. En naturlig fråga att ställa sig är, givet ett beräkningsproblem, om dess komplexitet är tillräckligt låg för att det i praktiken ska gå att lösa problemet på ett sådant sätt att komplexiteten inte skjuter i höjden. Sådana problem sägs vara hanterbara (på engelska "tractable"). Nästa naturliga fråga att ställa sig är vilka beräkningsproblem som är hanterbara. Att beräkna summan av två tal är självfallet ett hanterbart problem. Likaså existerar det effektiva metoder för att beräkna den kortaste vägen mellan två städer. Däremot känner man inte till någon effektiv metod för att undersöka huruvida det, givet $n$ städer och ett värde $k$, existerar en rutt som besöker alla städer som inte överskrider längden $k$, det så kallade handelsresandeproblemet (på engelska "the travelling salesman problem"). Däremot, som läsaren uppmanas testa, är det inte särskilt svårt att verifiera om en lösning till handelsresandeproblemet är korrekt eller ej, eftersom man i det fallet bara kan gå igenom rutten stad för stad, addera alla avstånd, och i slutändan se efter om den resulterande längden är kortare än k. Beräkningsproblem som man inte vet om de är hanterbara eller ej, men där det i likhet med handelsresandeproblemet alltid är möjligt att effektivt verifiera om en given lösning är korrekt, kallas för NP-fullständiga problem.

Man kan nu ställa sig frågan om det rent generellt sett är svårare att verifiera en lösning till ett beräkningsproblem än att beräkna en lösning från grunden. Denna fråga kallas i litteraturen för $\mathrm{P} \stackrel{?}{=}$ NP och är det viktigaste olösta problemet i teoretisk datalogi. $\mathrm{Om} \mathrm{P}=\mathrm{NP}$ så innebär det att det alltid är lika enkelt att beräkna en lösning till ett problem som det är att verifiera huruvida en lösning är korrekt. En praktisk konsekvens av detta är att en stor mängd med problem som man tidigare inte trott vara hanterbara i sådana fall skulle visa sig ha effektiva algoritmer. Exempelvis så skulle många befintliga krypteringsmetoder bli oanvändbara eftersom de bygger på antagandet att det inte är hanterbart att faktorisera stora heltal. I och med dessa stora praktiska konsekvenser, och det faktum att mer än 50 års forskning inte har resulterat i effektiva algoritmer för NP-fullständiga problem, anses 
det troligare att $\mathrm{P} \neq \mathrm{NP}$. Många av resulteten i denna avhandling bygger på antagandet att $\mathrm{P} \neq \mathrm{NP}$ och skulle således bli överflödiga om det mot förmodan skulle visa sig att $\mathrm{P}=\mathrm{NP}$.

Avhandlingens syfte är att studera komplexiteten för en klass av NPfullständiga beräkningsproblem som kallas för villkorsproblem (på engelska "constraint satisfaction problems"). En instans av ett villkorsproblem består av en mängd variabler och en mängd villkor som dessa variabler måste uppfylla. Frågan som ska besvaras är huruvida det är möjligt att tilldela värden till dessa variabler på ett sådant vis att alla villkor är uppfyllda. Det är alltså ett väldigt generellt beräkningsproblem, och genom att välja olika typer av villkor kan man formulera en stor mängd beräkningsproblem som villkorsproblem. Vi är intresserade av att jämföra komplexiteten för de villkorsproblem som är NP-fullständiga och därför tros vara extremt svåra att lösa. I avhandlingens första bidrag studerar vi algebraiska metoder som är användbara för att studera komplexiteten hos villkorsproblem. Dessa algebror kallas för starka partiella kloner (på engelska "strong partial clones"och skiljer sig drastiskt från de algebror som använts för att studera villkorsproblem tidigare. Den algebraiska teorin tillämpas sedan i avhandlingens andra del där vi bland annat identifierar det lättaste NP-fullständiga villkorsproblemet. Trots att detta problem är det lättaste NP-fullständiga villkorsproblemet bevisar vi att dess komplexitet på ett naturligt vis kan relateras till komplexiteten för andra välstuderade villkorsproblem. Vi upprepar sedan detta konststycke för två beräkningsproblem som är nära besläktade med villkorsproblem.

Vad är då syftet med att identifiera sådana typer av problem? I någon mening kan man säga att det lättaste NP-fullständiga villkorsproblemet skapar en stark avgränsning för hur lätta NP-fullständiga problem det är möjligt att konstruera. Utan detta resultat skulle man exempelvis kunna tro att en rimlig ansats för att bevisa att $\mathrm{P}=\mathrm{NP}$ skulle vara att skapa ett NP-fullständigt villkorsproblem med så låg komplexitet att det blir hanterbart. Vårt resultat visar att en sådan metod omöjligen kan fungera. Därför kan liknande studier ge en fingervisning om vad som skiljer NP-fullständiga problem, oavsett hur låg komplexitet de har, mot hanterbara problem. 



\section{Acknowledgements}

Writing this thesis has been a wild ride. And by wild ride, I mean that the writing process has consisted of late, nocturnal activities coupled with copious amounts of coffee, a lifestyle that I do not recommend anyone to try for extended periods of time. In retrospect, it is fair to say that the amount of work required to write this monograph slightly exceeded my original expectations.

There are several persons who deserve to be acknowledged. First of all, I want to thank the past and present members of the laboratory of theoretical computer science at Linköping University for providing a unique working environment. In particular, I want to thank Gustav Nordh for supervising my master's thesis, which served as a gateway to heavier sciences, and Peter Jonsson for excellent supervision and support during my time as a PhD student. Christer Bäckstrom, my secondary supervisor, also deserves acknowledgement for giving extensive feedback on the introductory chapter of this thesis, and for pointing out a variety of misconceptions of the English language. It is clearly too say, that any remainder errours are entirely my own falt.

I also want to extend my gratitude towards my co-authors that have not been mentioned so far: Johannes Schmidt, Hannes Uppman, Magnus Wahlström, and Bruno Zanuttini. Without your help and input this thesis would most likely not have obtained its current shape.

Last, I want to thank my friends and family for their continuous support and encouragement, and Maria, whose love brightens up even the bleakest day of the week. 



\section{List of Papers and Articles}

Parts of this thesis have previously been published in the following refereed publications:

- V. Lagerkvist, M. Wahlström and B. Zanuttini (2015). “Bounded Bases of Strong Partial Clones". In: Proceedings of the 45th International Symposium on Multiple-Valued Logic (ISMVL-2015), pages 189-194.

- P. Jonsson, V. Lagerkvist, J. Schmidt and H. Uppman (2014). “Relating the Time Complexity of Optimization Problems in Light of the Exponential-Time Hypothesis". In: Proceedings of the 39th International Symposium on Mathematical Foundations of Computer Science (MFCS2014), pages $408-419$.

- V. Lagerkvist and M. Wahlström (2014). "Polynomially Closed CoClones". In: Proceedings of the 44th International Symposium on MultipleValued Logic (ISMVL-2014), pages 85-90.

- V. Lagerkvist. "Weak Bases of Boolean co-clones" (2014). In Information Processing Letters 114(9), pages 462-468.

- P. Jonsson, V. Lagerkvist, G. Nordh and B. Zanuttini (2013). "Complexity of SAT Problems, Clone Theory and the Exponential Time Hypothesis". In: Proceedings of the 24th Annual ACM-SIAM Symposium on Discrete Algorithms (SODA-2013), pages 1264-1277. 


\section{Contents}

$\begin{array}{lll}\text { Abstract } & \text { iii }\end{array}$

Populärvetenskaplig sammanfattning v v

\begin{tabular}{|l|l}
\hline Acknowledgements & ix
\end{tabular}

List of Papers and Articles $\quad$ xi

Contents xii

\begin{tabular}{ll}
\hline Introduction & 1
\end{tabular}

$\mathbf{1}$ Introduction 3

1.1 Computational Complexity Theory . . . . . . . . . . . . . . 5

1.1.1 Computational Problems . . . . . . . . . . 5

$1.1 .2 \quad$ Asymptotic Complexity . . . . . . . . . . . . . . . . . . 8

1.1.3 Complexity Classes . . . . . . . . . . . . . . . . 8

1.2 Constraint Satisfaction Problems . . . . . . . . . . . . . . . . . 12

1.3 Universal Algebra and Clone Theory . . . . . . . . . . . . . . . . . . . . . 14

1.3.1 Examples of Algebras . . . . . . . . . . . . . . 14

1.3 .2 The Story of Clones . . . . . . . . . . . . . . . . . . 15

1.3.3 Clones and Constraint Satisfaction Problems . . . . . . 16

1.4 Main Contributions and Thesis Outline . . . . . . . . . . . . . 18

2 Preliminaries 23

2.1 Functions, Relations and Constraint Languages. . . . . . . . . 23 
2.2 The Constraint Satisfaction Problem . . . . . . . . . . . . . . 24

2.3 Clone Theory . . . . . . . . . . . . . . . . . . . . . 25

2.3 .1 Clones . . . . . . . . . . . . . . . . . . . . . . . 25

$2.3 .2 \quad$ Co-Clones . . . . . . . . . . . . . . . . . 27

2.3.3 The Galois Connection between Clones and Co-Clones 33

2.4 Partial Clone Theory . . . . . . . . . . . . . . . . . . . 36

2.4 .1 Clones of Partial Functions . . . . . . . . . . . 36

2.4 .2 Weak Partial Co-Clones . . . . . . . . . . . . . . 37

2.4.3 The Galois Connection between Strong Partial Clones and Weak Partial Co-Clones . . . . . . . . . . . . . . 38

II Properties of Strong Partial Clones 41

$3 \quad$ Weak Bases of Boolean Co-Clones 43

3.1 Introduction . . . . . . . . . . . . . . . . . . . . . 43

3.2 Preliminaries . . . . . . . . . . . . . . . . . . . 44

3.3 Minimal Weak Bases of Boolean Co-Clones . . . . . . . . . . 46

3.3 .1 The Finite Cases . . . . . . . . . . . . . . . . . . . 46

3.3 .2 The Infinite Cases . . . . . . . . . . . . . . . . . . 54

3.4 Summary and Conclusions $\ldots \ldots \ldots \ldots \ldots$

\begin{tabular}{|ll|}
\hline 4 & Polynomially Closed Co-Clones and Strong Partial Clones of In- \\
\hline & finite Order \\
\hline
\end{tabular}

4.1 Introduction . . . . . . . . . . . . . . . . . . . . . . . 57

4.2 Preliminaries . . . . . . . . . . . . . . . . . . . . . . 59

$4.3 \quad$ Polynomially Closed Co-Clones of Finite Order. . . . . . . . . 59

4.4 Polynomially Closed Co-Clones of Infinite Order. . . . . . . . 65

4.5 Superpolynomially Closed Co-Clones . . . . . . . . . . . . 65

4.6 Strong Partial Clones of Finite and Infinite Order . . . . . . . . . 69

4.6 .1 Strong Partial Clones of Finite Order . . . . . . . . . . 70

4.6 .2 Strong Partial Clones of Infinite Order . . . . . . . . . 72

4.7 Summary and Conclusions $\ldots \ldots \ldots \ldots \ldots$

5 Bounded Bases of Strong Partial Clones 77

5.1 Introduction $\ldots \ldots \ldots \ldots \ldots \ldots \ldots \ldots \ldots$

5.2 Preliminaries . . . . . . . . . . . . . . . . . . . . . 79

5.3 Bounded Bases . . . . . . . . . . . . . . . . . . . . . . 79

5.4 Closure of Bounded Bases . . . . . . . . . . . . . . . . 83

5.5 Determining Bounded Bases of Boolean Constraint Languages 85

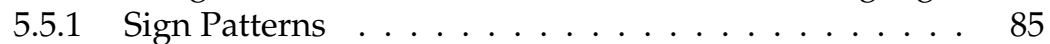

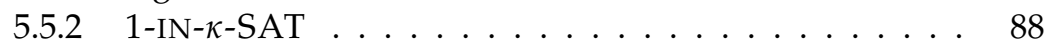

$5.5 .3 \quad \kappa$-SAT $\ldots \ldots \ldots \ldots \ldots$

5.6 Summary and Conclusions . . . . . . . . . . . . . 91 
6 Size-Preserving Reductions and the Exponential-Time Hypothesis 95

6.1 Problems Under Consideration . . . . . . . . . . . . . . . 95

6.2 Subexponential Time, Size-Preserving Reductions and the Exponential-Time Hypothesis $\ldots \ldots \ldots$. . . . . . . . . 96

\begin{tabular}{|lll}
7 & The Boolean Satisfiability Problem & 99
\end{tabular}

7.1 Introduction $\ldots \ldots \ldots \ldots \ldots \ldots \ldots$

7.2 Preliminaries . . . . . . . . . . . . . . . . . . . . . . 102

7.3 Partial Polymorphisms and CV-Reductions . . . . . . . . 103

7.4 The Easiest NP-complete SAT $(S)$ Problem . . . . . . . . . . . 105

7.5 Complexity Bounds for SAT $\left(\left\{R_{1 / 3}^{\neq \neq \neq}\right\}\right)$and Related Problems. 107

\begin{tabular}{ll|lll}
\hline 7.6 & Weak Partial Co-Clones Covering BR & $\ldots \ldots \ldots \ldots$ \\
\hline
\end{tabular}

7.6.1 Weak Partial Co-Clones below $\left\langle\Gamma_{1 / 3}\right\rangle_{\nexists} \ldots \ldots \ldots$. . . . 111

7.6.2 Weak Partial Co-Clones above $\left\langle\Gamma_{1 / 3}\right\rangle_{7} \mid \ldots \ldots . . . . .114$

7.7 LV-Reductions and Bounded-Degree Instances . . . . . . . 116

7.8 The Exponential-Time Hypothesis $\ldots \ldots \ldots$

$7.8 .1 \quad$ Two Notions of Sparsification . . . . . . . . . . . . . . . 121

\begin{tabular}{ll|lll}
7.8 .2 & Sparsification within $\mathcal{H}$ & $\ldots$ & $\ldots$
\end{tabular}

7.8.3 SAT $(\cdot)$ and the Exponential-Time Hypothesis . . . . . 127

7.9 Summary and Conclusions $\ldots \ldots \ldots \ldots$

8 Boolean Optimisation Problems 133

8.1 Introduction $\ldots \ldots \ldots \ldots \ldots \ldots \ldots \ldots$

8.2 Preliminaries . . . . . . . . . . . . . . . . . . . . . . . 135

8.3 The Easiest NP-complete MAX-ONES and VCSP Problems . . 136

8.3 .1 The MAX-ONES Problem $\ldots \ldots \ldots$. . . . . . . . 137

8.3 .2 The VCSP Problem. . . . . . . . . . . . . . . . . . . . 140

8.3 .3 The Broader Picture . . . . . . . . . . . . . . . . 141

8.4 Subexponential Time and the Exponential-Time Hypothesis . 142

8.5 Summary and Conclusions $\ldots \ldots \ldots \ldots \ldots$

\begin{tabular}{ll}
\hline IV Conclusion & 149
\end{tabular}

$9 \quad$ Final Thoughts 151

9.1 When are Strong Partial Clones of Infinite Order? . . . . . . . 152

9.2 Covering Results for Strong Partial Clones $\ldots . . . . . . . .152$

9.3 Easy Problems and Subexponential Time . . . . . . . . . . 152

$\begin{array}{ll}\text { Bibliography } & 155\end{array}$ 


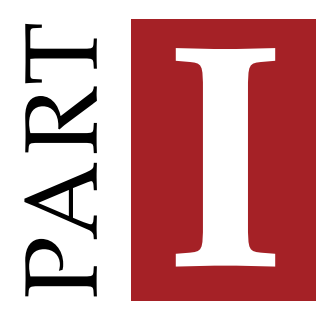

Introduction 



\section{Introduction}

- Apply you at the study during that you are young.

English as she is spoke

In this thesis we study the computational complexity of constraint satisfaction problems using techniques from partial clone theory. The purpose of this introduction is to give an intuition of the meaning behind the previous sentence, which, to any reader unfamiliar with this particular branch of theoretical computer science, is probably as nonsensical and whimsical as the introductory quote of this chapter. Let us start from the beginning with an example illustrating some of these concepts. The reader with a background in computer science can safely skip this introductory example.

Example 1.1. What do we mean by computational complexity? Assume that you are given a deck of shuffled cards and want to sort them in ascending order depending on the colour and suit of each card. For example, we could say that spades should be placed before hearts, that hearts should be placed before diamonds which in turn should be placed before clubs, and that the cards in each suit should be ordered according to their values. How would you do this? A rather natural way to approach this problem is to remove the first card from the shuffled deck and place it in a new deck. Then, for each remaining card in the shuffled deck, insert it in the correct position in the new deck. When the original deck of cards is empty, the second deck will contain the cards in sorted order. This systematic procedure is an example 
of an algorithm for the problem of sorting a deck of cards. How efficient is this algorithm, i.e., how long does it take to sort a deck of cards? To measure this we could e.g. implement this sorting procedure on a computer by representing the deck of card by a list of numbers from 1 to 52 . Such a program would compute the correct answer almost immediately. Does this mean that we have implemented an efficient algorithm? Not really. What if we instead want to sort a billion decks of cards? Then we would most likely not obtain an answer within a reasonable amount of time. Could this not be due to the fact that we run the program on a computer which is too slow? But, even if we increase the speed of the computer and manage to get an answer, this does not really tell us anything about the efficiency of the algorithm itself. To give a satisfactory answer to this question we have to go back to the drawing board and reason about the algorithm in more abstract terms. Assume that some operations, for example a comparison between two numbers, can be executed in constant time. Then, in the worst possible case, how many operations would we need to sort a list of numbers of arbitrary size? Let us denote the amount of numbers that we want to sort by $n$. First, we need to go through the list of numbers, and since the list in total contains $n$ numbers, this amounts to $n$ iterations. For each such iteration, we take the current number (corresponding to the first card in the deck) and insert it into the correct position in a second list of numbers (corresponding to the second deck of cards), which is initially empty. Now observe that in the worst possible case, this requires roughly $n$ operations, e.g., if only one number is remaining and we have to insert that number last in the sorted list. In total we therefore require roughly $n \cdot n=n^{2}$ operations in the worst case. While this is a quite coarse analysis, it at least gives a ballpark figure regarding the complexity of the sorting algorithm. For example, we see that for large values of $n$, say $n=1000000$, our algorithm is not going to perform very well. There are in fact much better sorting algorithms in the literature with a running time of roughly $n \cdot \log (n)$ operations in the worst case.

As evident from Example 1.1, given a problem and an algorithm for this problem, we are not particularly interested in how long a particular implementation takes to execute on a specific computer, but rather in the asymptotic behaviour of the complexity of the algorithm. In this thesis we are interested in studying the asymptotic complexity of certain computational problems that are believed to be intractable to solve. These notions will be defined in greater detail in Section 1.1. but, for the moment, let us merely state that certain kinds of problems are believed to require exponential time to solve. Exponential functions in general grow extremely fast. For example, if $f(n)=2^{n}$, then $f(300)$ is several orders of magnitudes larger than the estimated number of atoms in the observable universe. Despite this, many intractable problems are solvable much faster than $2^{n}$ time, and it is safe to say that we currently do not have a solid understanding of why, even among the intractable problems, some appear to be much easier to solve than others. We study this question in the context of a class of problems referred 
to as constraint satisfaction problems. The exact nature of these problems and their relevance is explained in Section 1.2, but for the moment we have to be contempt with the fact that it is a large and well-studied class of tractable and intractable problems with a variety of practical applications. The main question that we are interested in is whether we can give some kind of explanation of why some of these intractable problems appear to be so much easier to solve than others. We are therefore interested in comparing the time complexity of intractable problems, and obtaining properties of when it is possible to use an algorithm for one problem to solve another problem equally fast. Our main tool for accomplishing this is a branch of mathematics called universal algebra, and, in particular, a sub-branch of universal algebra that deals with algebras of partial functions over finite sets. Using such algebras, we are able to obtain a nuanced view of the time complexity of many intractable constraint satisfaction problems.

The remainder of this introduction, where we describe our results in greater detail, is split into four parts: in Section 1.1 we give a brief overview of complexity theory, in Section 1.2 we get acquainted with constraint satisfaction problems, in Section 1.3 we introduce some algebraic techniques used in this thesis, and, at last, in Section 1.4 we summarise our results and give an outline of the structure of this thesis.

\subsection{Computational Complexity Theory}

In this section we provide a bird's-eye view on the area of theoretical computer science referred to as computational complexity theory. The notation and terminology in this section essentially follows the introductory textbook by Papadimitriou [Pap94].

\subsubsection{Computational Problems}

We begin by giving a few examples of computational problems. Let us briefly return to the problem of sorting a list of numbers. This problem can be formally defined as the following computational problem.

\footnotetext{
SORTING

INSTANCE: A sequence $x_{1}, \ldots, x_{n}$ of natural numbers.

OвJестіVE: Find a permutation $\pi:\{1, \ldots, n\} \rightarrow\{1, \ldots, n\}$ such that $x_{\pi(i)} \leq x_{\pi(i+1)}$ for each $i \in\{1, \ldots, n-1\}$
}

This is a typical example of how one defines a computational problem in theoretical computer science. First, we specify exactly what constitutes an instance of the problem. Second, we specify the objective, the goal, of the problem. Note that the definition of the problem says absolutely nothing about how one should proceed to construct an algorithm for the problem; it merely states what should be computed, not how it should be computed. 
Clearly, there exist many different algorithms that produce the same answer, even if they may differ in complexity. For example, the sorting algorithm that we considered in Example 1.1 is called insertion sort in the literature, and as remarked, requires roughly $n^{2}$ operations in the worst case. Other sorting algorithms, such as merge sort, require roughly $n \cdot \log (n)$ operations in the worst case.

The problem of sorting a sequence of numbers is an example of a function problem since we are asked to compute a concrete permutation. Let us consider another example of a function problem. In the TRAVELLING SALESMAN PROBLEM (TSP) one is given $n$ numbers $1, \ldots, n$, representing cities, and for each pair $i, j$ of cities, a natural number $d_{i, j}$ which represents the distance between the two cities $i$ and $j$. The objective is then to find a tour of all cities such that the total distance is minimised.

THE TRAVELLING SALESMAN PROBLEM (TSP)

INSTANCE: A sequence $1, \ldots, n$ of natural numbers and a natural number, a distance, $d_{i, j}$ between every pair $i, j$ in this sequence.

OвJестіVE: Find a permutation $\pi:\{1, \ldots, n\} \rightarrow\{1, \ldots, n\}$ such that $\sum_{i=1}^{n} d_{\pi(i), \pi(i+1)}$ is minimal.

In this problem definition we tacitly assume that $\pi(n+1)=\pi(1)$. What is the complexity of the TSP problem? First observe that we can solve this problem in a brute force way by simply calculating all possible permutations over $n$ and take the one that minimises the sum of the distances. Such an algorithm would run in roughly $1 \cdot 2 \cdot \ldots \cdot(n-1) \cdot n=n$ ! time, which is clearly infeasible even for quite moderate values of $n$. Can we, similarly to the problem of sorting a sequence of numbers, devise a nice algorithm that solves the TSP problem in $n^{k}$ time for some reasonably small value of $k$ ? If this is not possible, can we at least find some $k \geq 1$ such that we can solve the problem in $n^{k}$ time, i.e., in polynomial time? Currently, no such algorithm is known, and it is widely believed that such algorithms are impossible to achieve. We describe this in greater detail in the next section.

Sometimes one is contempt with whether there exists a solution or not. Such problems are called decision problems and they will be the main focus of this thesis. Note that decision problems can be seen as function problems where the output is always 'yes' or 'no'. We could for example recast the TSP problem as a decision problem by instead asking whether there exists a tour which is smaller than or equal to a given value.

THE DECISION VERSION OF TSP (D-TSP)

INSTANCE: A natural number $k$, sequence $1, \ldots, n$ of natural numbers and a natural number, a distance, $d_{i, j}$ between every pair $i, j$ in this sequence. 
QUESTION: Does there exist a permutation $\pi:\{1, \ldots, n\} \rightarrow\{1, \ldots, n\}$ such that $\sum_{i=1}^{n} d_{\pi(i), \pi(i+1)} \leq k$ ?

We now introduce a few classical decision problems from the realm of propositional logic. If $V$ is a set of variables and $f$ a function from $V$ to $\{0,1\}$, then we define the function $h_{f}$ as $h_{f}(x)=f(x)$ and $h_{f}(\neg x)=1-f(x)$ for any $x \in V$.

\section{$k$-SAT}

INSTANCE: A set of variables $V$ and a set clauses of the form $\left(\ell_{1} \vee \ldots \vee\right.$ $\ell_{k}$ ), where $\ell_{i}=\neg x$ or $\ell_{i}=x$ for some $x \in V$.

QUESTION: Does there exist a function $f: V \rightarrow\{0,1\}$ such that $h_{f}\left(\ell_{1}\right)+$ $\ldots+h_{f}\left(\ell_{k}\right) \geq 1$ for each clause $\left(\ell_{1} \vee \ldots \vee \ell_{k}\right)$ ?

Polynomial-time algorithms are known for 1-SAT and 2-SAT. However, similar to the case of D-TSP, no polynomial-time algorithms have been discovered when $k \geq 3$. A related problem to $k$-SAT is the 1 -IN- $k$-SAT problem. The difference between the two problems is that we in the latter case require that exactly one literal is true in each clause.

\section{$1-I N-k-S A T$}

INSTANCE: A set of variables $V$ and a set clauses of the form $\left(\ell_{1} \vee \ldots \vee\right.$ $\ell_{k}$ ), where $\ell_{i}=\neg x$ or $\ell_{i}=x$ for some $x \in V$.

QuESTION: Does there exist a function $f: V \rightarrow\{0,1\}$ such that $h_{f}\left(\ell_{1}\right)+$ $\ldots+h_{f}\left(\ell_{k}\right)=1$ for each clause $\left(\ell_{1} \vee \ldots \vee \ell_{k}\right)$ ?

Again, both 1-IN-1-SAT and 1-IN-2-SAT are solvable in polynomial time, but the same is not believed to be true for $1-\mathrm{IN}-k$-SAT for any $k \geq 3$. Interestingly, this phenomena persists even if we restrict the problem to instances consisting only of positive clauses.

MONOTONE 1-IN-k-SAT

INSTANCE: A set of variables $V$ and a set clauses of the form $\left(x_{1} \vee \ldots \vee\right.$ $\left.x_{k}\right)$, where $x_{1}, \ldots, x_{k} \in V$.

Question: Does there exist a function $f: V \rightarrow\{0,1\}$ such that $f\left(x_{1}\right)+$ $\ldots+f\left(x_{k}\right)=1$ for each clause $\left(x_{1} \vee \ldots \vee x_{k}\right)$ ?

We have now seen quite a few examples of decision problems. However, all definitions in this section share a common problem: we have described their instances but so far have not mentioned how these should be represented. This depends on the underlying computational model, which we 
discuss in the next section. In many cases, the exact representation is not of great importance, but there are exceptions, as the following example highlights.

Example 1.2. How should an instance of D-TSP be represented? Let $n$ denote the number of cities, let $k$ denote the largest allowed distance, and let $d_{1,2} \ldots, d_{1, n} \ldots, d_{n, 1}, \ldots, d_{n, n-1}$ be the distances. A possible representation for this instance is then the sequence $\left(n, k, d_{1,2} \ldots, d_{1, n} \ldots, d_{n, 1}, \ldots, d_{n, n-1}\right)$. There is one glaring omission with this idea. How should the numbers in this sequence be represented? In general it is desirable to come up with a representation over a finite alphabet, for example by representing numbers as strings over $\{0,1\}$. This suggest that we have essentially two choices for representing a number: a unary representation or a binary representation. For example, the number 6 could be represented as the string 111111 or as the string 110 . Which representation is better? Since any number $n$ can be represented by at most $\log (n)$ bits, the unary representation in fact requires exponentially more space than the binary representation. If we let $[n]_{b}$ denote the binary representation of a number $n$ then a reasonable representation of a D-TSP instance is a sequence $\left([n]_{b},[k]_{b},\left[d_{1,2}\right]_{b} \ldots,\left[d_{1, n}\right]_{b} \ldots,\left[d_{n, 1}\right]_{b}, \ldots,\left[d_{n, n-1}\right]_{b}\right)$.

\subsubsection{Asymptotic Complexity}

Assume that we have a computational problem and have constructed a nice algorithm for solving this problem, and we now want to see how many operations this algorithm requires in the worst case. It is then convenient to ignore constant factors and terms in such expressions, since, asymptotically, they do not affect the complexity. Let $\mathbb{N}$ denote the set of natural numbers, i.e., $\mathbb{N}=\{0,1,2, \ldots\}$. Given two unary functions $f: \mathbb{N} \rightarrow \mathbb{N}$ and $g: \mathbb{N} \rightarrow \mathbb{N}$ we say that $g \in O(f)$ if there exists $n_{0} \in \mathbb{N}$ and $c \in \mathbb{N}$ such that $g(n) \leq c \cdot f(n)$ for all $n \geq n_{0}$. Intuitively, if $g \in O(f)$, then $g$ does not grow asymptotically faster than $f$. Similarly, we write $g \in \Omega(f)$ if $f \in O(g)$, which means that $f$ does not grow faster than $g$. Last, $g \in \Theta(f)$ means that $g \in O(f)$ and $g \in \Omega(f)$. The eagle-eyed reader might notice two problems with these notions. First, if one e.g. uses the number of bits required to represent an instance in order to measure the complexity, then $f$ and $g$ have to be defined as partial functions, since it could be the case that $n \in \mathbb{N}$ but that there does not exist any instance of the problem whose representation requires exactly $n$ bits. Second, we sometimes use functions such as $\log (n)$ whose range is not equal to $\mathbb{N}$. If such a function $f$ appear in the above context then $f(n)$ is treated as a shorthand for $\max (\lceil f(n)\rceil, 0)$.

\subsubsection{Complexity Classes}

A complexity class is a set of problems. We could for example define a complexity class by letting it consist of all decision problems. In the literature this 
class is sometimes denoted ALL. Unfortunately, we do not really gain any insight by using the class ALL since it trivially includes all decision problems, and it is therefore common to consider complexity classes consisting of problems sharing certain properties. Frequently studied properties are time and space, which in this context means the number of operations or the amount of memory required to solve a problem. In this thesis we concentrate on the former, but before we can define the complexity classes that will play a central role, we need to make a detour and explain our underlying model of computation.

\section{Turing Machines}

Computing devices are certainly nothing new. Examples from ancient history include the Antikythera mechanism used for predicting astronomical observations, and abacuses, counting frames for performing arithmetical operations. These devices were constructed with a few very limited tasks in mind and do not fit the notion of universal computation that we normally associate with modern computers. The design of the first general purpose computer is normally attributed to Charles Babbage, who in 1837 described the so-called analytical engine. What made this computer different from the Antikythera mechanism and other primitive computing devices? This question was not answered until almost a hundred years later in Alan Turing's seminal paper On computable numbers with an application to the Entscheidungsproblem. What Alan Turing realised was that a seemingly extremely simple computer was sufficient to realise all imaginable forms of computation. A similar, and in fact equivalent, notion of universal computability was discovered independently by Alonzo Church.

For our purposes we see a Turing machine as a simple computer, equipped with a tape, that can be stretched arbitrarily long (so that we do not run out of space in the middle of a computation), and a head. Each cell in the tape can hold a symbol from a predetermined finite language. The program of the Turing machine consists of instructions of the form: if the machine is in a state and reads a symbol in the current cell, then write a new symbol in this cell and either (1) move the head to the left, (2) do not move the head, or (3) move the head to the right. These instructions have to be precise enough so that the Turing machine, for any possible state and symbol, always knows what the next step is. The Turing machine then patiently reads a single cell off the tape as input, writes a symbol on the cell, and moves the head according to its program. The machine halts if it ends up in either of the three special states YES, NO, or HALT. A Turing machine is said to decide a decision problem if, for every instance of the problem which is written on the tape in a suitable encoding, it correctly ends up in the YES state if and only if the instance is a yes-instance. 


\section{The Complexity Class P}

We let $\mathrm{P}$ be the class of decision probles decidable by a Turing machine using at most polynomially many steps (with respect to the size of the input). Problems in P are sometimes said to be tractable. Hence, complexity is measured with respect to the number of operations, and not with respect to CPU speed, which is not a meaningful concept for Turing machines. For example, $\mathrm{P}$ contains the problem 2-SAT considered in the previous section, but it is currently not known whether D-TSP or $k$-SAT for $k \geq 3$ is included in P or not. When proving that a particular problem resides in $\mathrm{P}$ we usually do not explicitly construct a Turing machine that decides this problem in polynomial time. Instead, we provide a polynomial-time algorithm in an informal language which is precise enough to be converted into a Turing machine without any ambiguity.

\section{The Complexity Class NP}

We now want to define a complexity class where the problems D-TSP and $k$-SAT are included. For this we need the concept of a nondeterministic Turing machine. We will omit the exact details, since they are not central to our study and can be found in any introductory textbook on computational complexity, see e.g. Chapter 2 in Papadimitriou [Pap94]. A nondeterministic Turing machine is defined in essentially the same way as an ordinary Turing machine. The exception is that, when the nondeterministic Turing machine enters a particular state and reads a symbol, it can end up in a multitude of new states; some of which might lead to accepting computations, and some of which might lead to utter gibberish. The nondeterministic Turing machine is said to decide a decision problem if for every yes-instance of the problem, there is some nondeterministic choice which leads to the YES state, and for every no-instance of the problem, every possible nondeterministic choice ends up in the NO state. Hence, a nondeterministic Turing machine might at a particular state have many different choices, but this does not matter as long as a single one of these ends up being a correct choice. We let NP denote the set of decision problems decidable by a nondeterministic Turing machine using at most polynomially many steps. An equivalent way of defining the class NP is to define it as the set of decision problems where certificates, i.e. proofs, to yes-instances are verifiable in polynomial time. For example, take an instance of the D-TSP problem and let $\pi$ be a permutation on $\{1, \ldots, n\}$. We can calculate the sum $\sum_{i=1}^{n} d_{\pi(i), \pi(i+1)}$ in $O(n)$ time and check whether this result is smaller than or equal to $k$. Hence, to solve this problem in nondeterministic polynomial time we can simply

1. write an arbitrary string of symbols (where the length is bounded by the length of the input),

2. check whether the resulting string is an encoding of a permutation $\pi$ on $\{1, \ldots n\}$, and 
3. answer yes if the permutation $\pi$ is a valid answer and otherwise no.

The above algorithm showcases the strength of nondeterministic computation since the Turing machine in this case in essence only has to 'guess' an answer and check whether it is valid (in polynomial time). Similarly, one can prove that $k$-SAT is solvable in nondeterministic polynomial time. Hence, both D-TSP and $k$-SAT are included in NP. The question of whether every problem decidable in nondeterministic polynomial time is also decidable in polynomial time is referred to as the $\mathrm{P} \stackrel{?}{=} \mathrm{NP}$ question and it is the most important open question in theoretical computer science. It is interesting to note that, despite the apparent difficulty of resolving this conjecture, there exists hundreds of claimed proofs of both $\mathrm{P}=\mathrm{NP}$ and $\mathrm{P} \neq \mathrm{NP}$ [Woe].

The reader might now ask why we went through all this trouble and defined the complexity class NP which, in constrast to $\mathrm{P}$, does not correspond to a realistic model of computation. Why not, for example, instead define a complexity class consisting of all problems decidable by a Turing machine using exponential time? Such a complexity class does in fact exist. In the literature it is called EXPTIME. It includes both D-TSP and $k$-SAT, and it is known that $\mathrm{P} \subset$ EXPTIME (note the proper inclusion). However, this still does not imply that $k$-SAT $\notin \mathrm{P}$. But there is a more fundamental reason why we believe that the complexity class NP captures the essence of D-TSP and $k$-SAT better than the class EXPTIME. It is known that both $k$-SAT and D-TSP are not just ordinary members of NP, but that they are as difficult to solve as any other problem in NP.

Before we can explain why this is the case we need a notion of mapping one problem to another problem. A polynomial time many-one reduction between two problems $X$ and $Y$ is a function from the instances of $X$ to the instances of $Y$ such that, for each instance $x$ of $X, f(x)$ is a yes-instance of $Y$ if and only if $x$ is a yes-instance of $X$. A problem $X$ is said to be NP-hard if every problem in NP is polynomial-time many-one reducible to $X$. The problem $X$ is in addition said to be NP-complete if it is also included in NP. Both D-TSP and $k$-SAT, $k \geq 3$, are known to be NP-complete. What does this mean in practice? Assume that we stumble upon an algorithm that solves 3-SAT in polynomial time. Then, since 3-SAT is NP-complete, we can solve D-TSP in polynomial time, too, by the following algorithm.

1. Let $f$ be a polynomial-time many-one reduction from D-TSP to 3-SAT.

2. Let $I$ be an instance of D-TSP.

3. Compute $f(I)$ and answer yes if $f(I)$ is a yes-instance of 3-SAT and no if $f(I)$ is a no-instance of 3-SAT.

In fact, we can solve every problem in NP in polynomial time by reusing our algorithm for 3-SAT. Hence, finding a polynomial-time algorithm for any NP-complete problem implies that we also have polynomial-time algorithms for all NP-complete problems, which implies that $\mathrm{P}=\mathrm{NP}$. Note, 


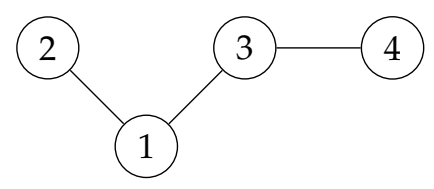

Figure 1.1: A simple connected graph.

however, that even if we could solve 3-SAT in polynomial time then this would not imply that we could solve every problem in EXPTIME in polynomial time. This suggests that the slightly non-intuitive complexity class NP is a much better fit for problems such as $k$-SAT and D-TSP than EXPTIME.

\subsection{Constraint Satisfaction Problems}

The constraint satisfaction problem (CSP) is the problem of determining whether a set of constraints has a solution. What is meant by a constraint in this context? Assume that we have system of linear equations over the rational numbers $\mathbb{Q}$ of the form

$$
\left\{\begin{aligned}
2 x+y+3 z & =10 \\
x+y+z & =6 \\
x+3 y+2 z & =13
\end{aligned}\right.
$$

Each linear equation constrains the variables $x, y$, and $z$ in the sense that the possible values for each variable is reduced. Hence, a constraint takes a collection of variables ranging over some set of values, a domain, and constrain the possible values that the variables can have in a solution. Given a collection of such constraints over some variables we are then interested in whether it is possible to assign values to the variables from the domain, in such a way that no constraint, e.g., $2 x+y+3 z=10$, is violated. In this thesis we are only concerned with variables ranging over a finite domain, and we therefore always assume that the domain is a finite set of natural numbers. Moreover, we are not only interested in arithmetical constraints appearing in linear equations, but in constraints that can be expressed by arbitrary relations over the domain. In a philosophical sense, a relation represents a property that holds between some given entities, for example the relation father that holds between a father and his children. In a mathematical sense a relation with $k$ arguments is defined as a subset of the $k$-ary cartesian product of the domain $D$. This means that the unary relations are subsets of $\{(x) \mid x \in D\}$, binary relations subsets of $\{(x, y) \mid x, y \in D\}$, and so on.

Let us take a concrete example to illustrate these concepts. Assume that we have a set 1,2,3,4 of nodes, as in Figure 1.1. with the interpretation that the nodes represent cities, and that two cities have an edge between them if they are directly connected with a road. We can model this by using a binary relation connected with the interpretation that connected $(x, y)$ is true if the two cities $x$ and $y$ have a connection between them. The 
graph in Figure 1.1 could then be represented by the relation connected $=$ $\{(1,2),(2,1)(1,3),(3,1),(3,4),(4,3)\}$. Assume then that we are given the two constraints connected $(x, y)$, connected $(x, z)$. For which values of $x, y$ and $z$ will these two constraints be satisfied? Two possible answers are when $x=1, y=2, z=1$, and when $x=1, y=3, z=4$. On the other hand, if $x=1, y=2$, and $z=3$, then the constraints are not satisfied since there is no connection from 2 to 3 .

More generally, we define a constraint over a set of variables $V$ and a $k$-ary relation $R$ to be an expression of the form $R\left(x_{1}, \ldots, x_{k}\right)$ where $x_{1}, \ldots, x_{k} \in V$. We now have everything in place in order to define the constraint satisfaction problem over a finite domain $D$.

\section{THE CONSTRAINT SATISFACTION PROBLEM OVER $D$}

INSTANCE: A set of variables $V$ and a set of constraints over $D$ of the form $R\left(x_{1}, \ldots, x_{k}\right)$, where $x_{1}, \ldots, x_{k} \in V$ and $R$ is a $k$-ary relation over $D$. QUESTION: Does there exist a function $f: V \rightarrow D$ such that $\left(f\left(x_{1}\right), \ldots, f\left(x_{k}\right)\right) \in R$ for each constraint $R\left(x_{1}, \ldots, x_{k}\right)$ ?

We say that an instance is satisfiable if such a function exists and unsatisfiable otherwise. This is the most complicated decision problem that we have encountered so far in this thesis. Hence, let us pause for a moment and try to explain how this definition fulfils the intuition outlined in the earlier examples. An instance consists of a set of variables ranging over the domain $D$, and a set of constraints restricting the values of the variables. The goal is then to check whether it is possible to assign values to the variables in such a way that each constraint is satisfied. If we briefly return to the example in Figure 1.1 then the set of constraints $\{$ connected $(x, y)$, connected $(x, z)\}$ is a CSP problem over the variables $\{x, y, z\}$ over the domain $\{1,2,3,4\}$. As remarked, this instance is satisfiable, since if we let $f(x)=1, f(y)=3$ and $f(z)=4$, then $(f(x), f(y))=$ $(1,3) \in$ connected and $(f(y), f(z))=(3,4) \in$ connected. On the other hand, the instance $\{$ connected $(x, y)$, connected $(x, z)$, connected $(x, x)\}$, is unsatisfiable since the constraint connected $(x, x)$ can never be satisfied.

Given the very general formulation of the CSP problem it should not come as a surprise that it is NP-complete. We can in fact prove this in a very straightforward manner by exploiting the fact that the MONOTONE 1-IN-3-SAT problem from Section 1.1.1 is NP-complete.

Theorem 1.3. CSP is NP-complete for every finite domain larger than one.

Proof. First, note that the finite-domain CSP problem is included in NP since we, given an assignment of variables, can go through every constraint and check whether it is satisfied by the assignment, which takes polynomial time with respect to the size of the instance. Second, note that we only have to prove that the CSP problem over the Boolean domain is NP-hard, 
since each such problem can be seen as a CSP problem over a larger domain where the extra values are not used. Consider the relation $R_{1 / 3}=$ $\{(0,0,1),(0,1,0),(1,0,0)\}$ over $\{0,1\}$ and note that a constraint $R_{1 / 3}(x, y, z)$ is true if and only if exactly one of the variables is equal to 1 . We can now give a polynomial-time many-one reduction from MONOTONE 1-IN-3-SAT to CSP as follows: given an instance of MONOTONE 1-IN-3-SAT replace every clause $(x \vee y \vee z)$ with the constraint $R_{1 / 3}(x, y, z)$. It is then trivial to see that the resulting instance is satisfiable if and only if the original instance is satisfiable.

An active area of research is to find tractable subclasses of the CSP problem. A common restriction, which is our main interest in this thesis, is to only allow constraints from a particular set of relations, a constraint language. This parameterized version of the CSP problem will be formally defined in Section 2.2 in Chapter2 It is known that, for constraint languages defined over Boolean or ternary domains, such problems are always either in P or are NPcomplete [Sch78; Bul06]. Why is this interesting? Perhaps contrary to intution, it is known that there exists problems in NP not solvable in polynomial time but which are not NP-hard (assuming $\mathrm{P} \neq \mathrm{NP}$ ) [Lad75]. Such problems are said to be NP-intermediate. Hence, a priori, we cannot take dichotomy theorems that separates tractable problems from NP-complete problems for granted.

\subsection{Universal Algebra and Clone Theory}

In this section we explain the algebraic method that we use to study the complexity of constraint satisfaction problems. All concepts will be defined formally in Chapter 2. here we concentrate on explaining the intuition behind them.

\subsubsection{Examples of Algebras}

First of all, what is an algebra? This is not a clear-cut concept. In the most general sense, the word algebra is used to denote a mathematical structure consisting of symbols and rules for manipulating those symbols. We begin with a few examples of such structures that are usually referred to as algebras.

Example 1.4. In elementary algebra the vocabulary consists of numbers, for example all natural numbers or all rational numbers, and variables, typically denoted $x, y, z$. One can then formulate various arithmetical laws such as $x+y=y+x$ (addition is associative) and $x(y+z)=x y+x z$ (distributivity), or constraints such as $2 x=y$.

Even though the example above is rather trivial and informal, we have at least obtained some intuition of what constitutes an algebra: we have a domain such as all natural numbers in mind, we have operations such as 
addition and multiplication, and when formulating sentences in the algebra we often use symbols as placeholders for concrete values in the domain. Let us consider two more examples of algebras.

Example 1.5. A magma consists of a set $D$ and a binary operator $\cdot: D \times D \rightarrow$ $D$. A semigroup is a magma where the binary operator - is associative, i.e., $(x \cdot y) \cdot z=x \cdot(y \cdot z)$ for all $x, y, z \in D$. A monoid is a semigroup with an identity element $i \in D$ satisfying $i \cdot x=x \cdot i=x$ for all $x \in D$.

Example 1.6. A lattice consists of a set $D$ and two binary operators $\sqcup: D \times$ $D \rightarrow D$ and $\sqcap: D \times D \rightarrow D$ satisfying the following axioms for all $x, y, z \in$ $D$ [BS81].

1. $x \sqcup y=y \sqcup x$ and $x \sqcap y=y \sqcap x$ (commutative laws),

2. $x \sqcup(y \sqcup z)=(x \sqcup y) \sqcup z$ and $x \sqcap(y \sqcap z)=(x \sqcap y) \sqcap z$ (associative laws),

3. $x \sqcup x=x$ and $x \sqcap x=x$ (idempotent laws),

4. $x=x \sqcup(x \sqcap y)$ and $x=x \sqcap(x \sqcup y)$ (absorption laws).

One example of a lattice is the two operators $\wedge$ and $\vee$ on the Boolean domain $\{0,1\}$. It is for example not so difficult to verify that the commutative laws hold since $x \vee y=y \vee x$ and $x \wedge y=y \wedge x$ for any $x, y \in\{0,1\}$.

The examples above are certainly more complicated than Example 1.4 and might appear a tad abstract to any reader unfamiliar with such definitions. The takeaway message is that the algebras considered are not concrete algebras since neither the domain nor the operators are specified. Instead, they are collections of algebras satisfying certain conditions on the operators, e.g. that they are associative. The branch of mathematics studying properties of algebras with axioms similar to those in Example 1.5 and Example 1.6 is called abstract algebra, and some well-studied algebraic structures besides those already mentioned are groups, rings, and fields. We will not delve deeper into the mysteries of abstract algebra since it is not central for our purposes, but instead turn our attention towards universal algebra.

\subsubsection{The Story of Clones}

In this thesis we need algebras where no particular restrictions are imposed on the set of operations. This branch of mathematics is called universal algebra |BS81], and it generalises abstract algebra in the sense that we do not e.g. require that operators are associative and binary, that the domain contains an identity element, and so on. More specifically, we will consider functions defined on finite subsets of the natural numbers. A clone is a set of such functions with the property that, whenever we take some functions in the clone and compose them in various ways, then the resulting function is also included in the clone. By composing, we mean that one is allowed to combine several simpler functions in order to create a new function. This is similar to 
digital circuit theory where one can construct new logical gates by connecting the output of one or more gates to the input to another gate, or how one can use the result of a function as input to another function in a programming language. This closure property is the only restriction that we impose; otherwise a clone can consist of any functions that we can imagine. Most likely, this definition of a clone is a thorough disappointment to the reader with a vivid imagination, since it does not bear any resemblance with how the term 'clone' is used in more natural contexts. To the best of the author's knowledge, the term clone was first used in this mathematical context by Cohn [Coh81]. Other terms that have been used for similar mathematical objects are function algebras and iterative Post algebras.

Example 1.7. Let $D$ be a finite set of natural numbers. Then the set consisting of all functions over $D$ is a clone since it is trivially closed under composition of functions. Similarly, one can verify that the set of all functions satisfying $f(x, \ldots, x)=x$ for all $x \in D$ is a clone, the set of all idempotent functions.

There are many beautiful results in the literature regarding clones. Two of the most famous ones are Rosenberg's classification of all maximal clones [Ros70] and his classification of minimal clones [Ros86]. For more examples of clones and a discussion of open questions we refer the reader to Kerkhoff et al. [KPS].

\subsubsection{Clones and Constraint Satisfaction Problems}

Why are clones interesting at all in the context of theoretical computer science and complexity theory? In the end of the 1960s the American mathematician David Geiger published an article titled Closed Systems of Functions and Predicates [Gei68]. Little did he know that, across the pacific ocean, four Soviet mathematicians had independently discovered almost the same thing [BKKR69a: BKKR69b]. So what was the fuss all about? To understand this we first need the concept of a Galois connection. In the words of Marcel Erné [DEW13],

'Galois connections provide the order- or structure-preserving passage between two worlds of our imagination.'

This gives us the intuition that a Galois connection somehow relates two different classes of objects, but, of course, we have to be a bit more precise than this. Assume that we have two sets $A$ and $B$ which are both equipped with a way of ordering their respective elements. Let us denote the ordering over the set $A$ by $\leq_{A}$ and the ordering over the set $B$ as $\leq_{B}$. Then, a Galois connection consists of two mappings $f: A \rightarrow B$ and $g: B \rightarrow A$ which tell us how to get from the set $A$ to the set $B$, and vice versa. But $f$ and $g$ are not just any mappings, they have to respect the two orderings $\leq_{A}$ and $\leq_{B}$ in such a way that, if $a \in A$ and $b \in B$ then $f(a) \leq_{B} b$ if and only if $a \leq_{A} g(b)$. The two mappings $g$ and $f$ are said to be the dual upper adjunction and dual 


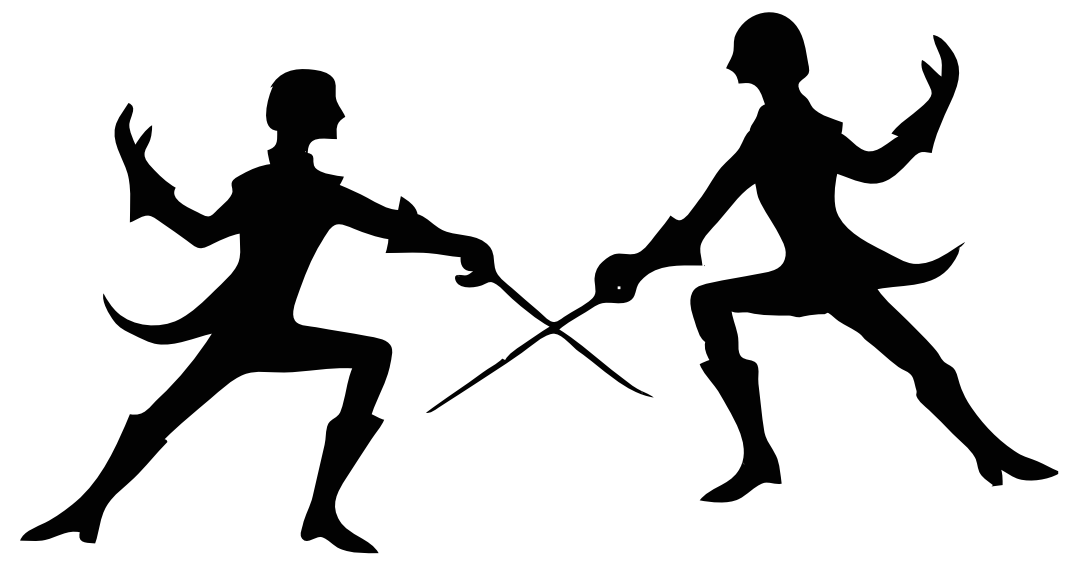

Figure 1.2: A visualisation of a Galois connection consisting of two dual adjunctions.

lower adjunction, respectively. See Figure 1.2 for a visualisation of the concept of a Galois connection consisting of two dual adjunctions. An interesting property of a Galois connection is the following observation: assume that $A$ has a maximal element $\max _{A}$ and that $B$ have a minimal element $\min _{B}$. Then a Galois connection between $A$ and $B$ will map $\max _{A}$ to $\min _{B}$ and $\min _{B}$ to $\max _{A}$. Hence, a 'large' element in $A$ will be mapped to a 'small' element in $B$. See Figure 1.3 for a visualisation of this mapping 1 . Why is this useful? Assume that we want to study properties of the elements in $A$ and that these properties are more complicated to study for 'large' elements in $A$. Then we can exploit a Galois connection between $A$ and $B$, and instead of studying the 'large' elements in $A$, we study these properties with respect to the 'small' elements in $B$, which is sometimes considerably simpler.

In the context of clones, Geiger [Gei68] and, independently, Bodnarchuk et al. [BKKR69a; BKKR69b], discovered that there exists a Galois connection between clones and sets of relations satisfying certain closure properties, $\mathrm{CO}$ clones. This implies that we can study properties of clones by studying their relational representations, and study relations by their functional representations. Since constraint satisfaction problems are defined in terms of relations, the Galois connection between clones and co-clones suggest that it is possible to study properties of constraint satisfaction problems by studying properties of clones. This rather simple observation was in fact not realised until the end of the 1990s by Jeavons et al. [JCG97], and it was the inception of the so-called algebraic approach for studying the computational complexity of constraint satisfaction problems. More precisely, Jeavons et al. proved that the complexity of a constraint satisfaction problem, whether it is in $\mathrm{P}$ or is $\mathrm{NP}$-complete, in many cases can be determined by the absence or presence

\footnotetext{
${ }^{1}$ Both Figure 1.2 and Figure 1.3 were kindly provided by Marcel Erné|DEW13. Ern85].
} 


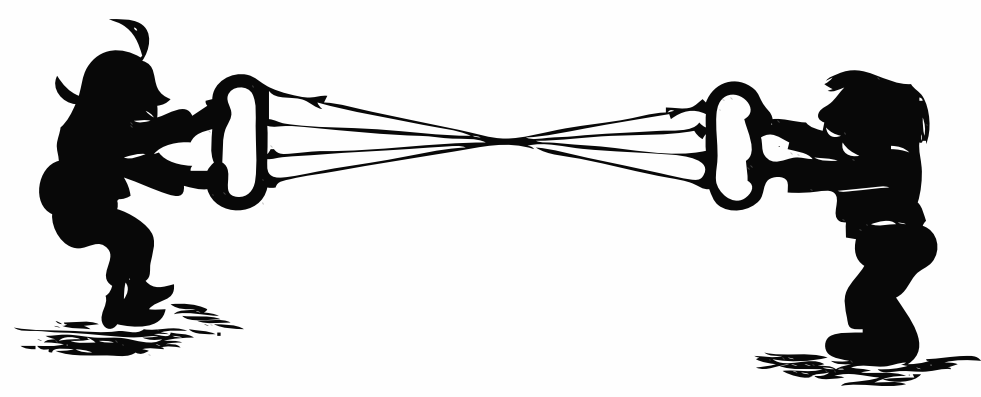

Figure 1.3: A visualisation of the mappings between elements in a Galois connection between two partially ordered sets.

of certain functions in the clone corresponding to the constraint satisfaction problem. Sometimes, due to the inverse nature of a Galois connection, such results are much easier to prove using properties of functions instead of using properties of relations.

However, as already remarked, merely stating that a problem is NPcomplete does not offer any insight into the complexity of the problem, except that it is not likely to be solvable in polynomial time. To study the complexity of NP-complete problems in greater detail we are in need of something more refined than the Galois connection between clones and co-clones. In this thesis we consider clones consisting of partial functions. As a simple example of a partial function, consider the division operator $\frac{x}{y}$ where $x \in \mathbb{N}$ and $y \in \mathbb{P}$, i.e., $x$ is a natural number and $y$ is a natural number distinct from 0 . Hence, $\frac{x}{0}$ is undefined for every $x \in \mathbb{N}$, since division is not a meaningful operation when the denominator is 0 . By using partial functions instead of total functions, one obtains a strong partial clone. There is a Galois connection between such strong partial clones and sets of relations satisfying certain closure properties, proved already in the article by Geiger [Gei68], and later developed by Romov |Rom81]. All these concepts will be clearly defined in Chapter 2. For the moment, the gist of this approach is that we can study the computational complexity of NP-complete CSP problems in a more nuanced way by considering algebras of partial functions (strong partial clones) instead of algebras of total functions (clones).

\subsection{Main Contributions and Thesis Outline}

We can now explain the title and topic of this thesis in a more precise way. Our main focus is constraint satisfaction problems and related problems, and we are interested in studying their computational complexity. To study this we use algebras of partial functions called strong partial clones, which al- 
lows us to give a very nuanced view of the worst-case time complexity of constraint satisfaction problems. So how does the thesis subtitle 'limitations and applications' fit into all of this?

The first technical contribution of this thesis, Part II is an investigation of properties of strong partial clones. Here, we first apply a technique from Schnoor and Schnoor [SS08] for obtaining relations with many interesting properties, so-called weak bases, that will play a central role in this thesis. We then proceed to show that strong partial clones are extremely complicated structures in the context of constraint satisfaction problems. In essence, we prove that for almost all constraint languages that we are interested in for practical purposes, the corresponding strong partial clones cannot be generated by any finite set of partial functions. In plain English, this means that our algebras are much more complicated than what one could possibly expect. This is a rather negative result, hence the term 'limitations' in the title of this thesis. On the bright side, we then change our minds and prove that it is possible to describe all such strong partial clones with finite sets of partial functions. We do this, not by bending the laws of logic, but by changing the standard method of functional composition in an algebra to a stronger notion.

To fulfil the promise of 'applications' in the title of this thesis, we in Part III switch gear and concentrate on studying the worst-case time complexity of constraint satisfaction problems. As hinted in the previous section, strong partial clones are indeed useful for this. For the CSP problem we prove the following: assume that you have two NP-complete CSP problems $A$ and $B$ and that you have a concrete algorithm for $B$ that runs in time $O\left(c^{n}\right)$ for some constant $c$ (where $n$ denotes the number of variables). Then, if the two strong partial clones corresponding to the relations used in $A$ and $B$ satisfy certain conditions, you can solve the CSP problem $A$ using the algorithm for the CSP problem $B$ and obtain the exact same $O\left(c^{n}\right)$ running time. Hence, for any conceivable algorithm that we can construct for problem $B$, we can directly reuse it and solve problem $A$ equally fast, just by comparing the algebras corresponding to the problems. However, recall from the previous paragraph that we in Part II of this thesis prove that such strong partial clones in general are extremely complicated. This suggests that this result alone is not sufficient to give us any new insight into the worst-case time complexity of CSP problems. However, what a weak base gives us is a CSP problem with the property that you can solve the problem using any algorithm for any NP-complete CSP problem, and obtaining the exact same worst-case running time. We use this fact to find an NP-complete Boolean CSP problem with the lowest possible worst-case time complexity. With the help of this problem we then relate the complexity of Boolean CSP problems to the exponential-time hypothesis, which is a well-known complexity conjecture [IPZ01]. Loosely, this conjecture states that there is sharp limit on how much it is possible to improve exponential-time algorithms for 3-SAT. We prove that this conjecture holds if and only if such sharp limits occur for all NP-complete Boolean CSP problems. Are such results limited to CSP or is it 
possible to obtain analogous results for similar problems? We show that this is indeed possible for two optimisation variants of the Boolean CSP problem, the maximum ones problem (MAX-ONES) and the valued constraint satisfaction problem (VCSP).

We now describe each part and chapter of this thesis in greater detail. In some cases we allow ourselves to use notation that has not yet been defined.

\section{Part I}

Apart from this introduction, this part of the thesis contains the preliminaries chapter where we formally define all concepts discussed so far. The most central terms that will be defined are clones, strong partial clones, co-clones, weak partial co-clones, polymorphisms, partial polymorphisms, Galois connections, and the parameterized $\operatorname{CSP}(S)$ problem. The reader who is already familiar with these concepts is still advised to consult Section 2.1. where some simplifying notation is introduced, and Section 2.3.2. where an important class of constraint languages is defined.

\section{Part II}

In this part of the thesis we study properties of strong partial clones. These results are in many cases of independent algebraic interest. We begin in Chapter 3 where we use results from Schnoor and Schnoor [SS08] in order to obtain simple weak bases for all Boolean co-clones. Given a clone C, a weak base is in this context a relational description of the largest element in the set of strong partial clones where the total component equals $C$. In Chapter 4 we study polynomially closed co-clones. Intuitively, this means that a polynomial number of variables is sufficient to define every relation in the co-clone via logical definitions consisting of existential quantification, conjunction and equality constraints. For Boolean co-clones of finite order this notion exactly coincides with the question of whether the corresponding algebra has few subpowers [BMMVW06]. Interestingly, for Boolean co-clones of infinite order, the opposite is true: they are polynomially closed even though the corresponding algebras have many subpowers [BMMVW06]. Using this classification we then prove the following theorem: let $S$ be a finite constraint language such that its corresponding clone can be generated by a finite set of unary functions - then the strong partial clone corresponding to $S$ is always of infinite order. This is a strong negative result since it implies that we cannot obtain finite bases of a significant number of the strong partial clones that are of interest when studying NP-complete CSP problems. Despite this, we in Chapter 5 develop a novel technique for representing such strong partial clones of infinite order by a finite set of partial functions. This might seem contradictory, but we accomplish this by introducing a stronger closure operator than functional composition. Hence, even though the strong partial clones of interest in this thesis are of infinite order it is still possible to describe them using finite sets of partial functions. 


\section{Part III}

In Part III we apply the theory studied and developed in Part II We are interested in studying constraint satisfaction problems with respect to polynomial-time many-one reductions that do not change the structure of instances in a dramatic way. More specifically, we in Chapter 6 introduce two kinds of polynomial-time many-one reductions: constant variable reductions and linear variable reductions. In the former, only a constant number of new variables are allowed to be introduced, while a linear amount is allowed in the latter case. Constant variable reductions allow us to study the complexity of CSP up to $O\left(c^{n}\right)$ time complexity, where $n$ denotes the number of variables in a given instance, while linear variable reductions allow us to study the complexity of a problem up to subexponential complexity. In Chapter 7 we then study the complexity of the Boolean $\operatorname{CSP}(S)$ problem $(\operatorname{SAT}(S)$ ) with respect to constant variable reductions and linear variable reductions. Given two finite constraint languages we first prove that if $\operatorname{pPol}(S) \subseteq \operatorname{pPol}\left(S^{\prime}\right)$, where $\mathrm{pPol}(S)$ (respectively $\mathrm{pPol}\left(S^{\prime}\right)$ ) is the strong partial clone corresponding to $S$ (respectively $S^{\prime}$ ), then there exists a constant variable reduction from $\operatorname{CSP}\left(S^{\prime}\right)$ to $\operatorname{CSP}(S)$. This proof is not complicated, but when combined with the concept of weak bases from Part II it almost immediately gives rise to a rather unexpected result: there exists a Boolean relation $R_{1 / 3}^{\neq \neq \neq}$such that (1) $\operatorname{SAT}\left(\left\{R_{1 / 3}^{\neq \neq \neq}\right\}\right)$is NP-complete and (2) there does not exist any NP-complete $\mathrm{SAT}(S)$ problem with strictly lower worst-case time complexity. We refer to this problem as the easiest NP-complete SAT problem. Moreover, the relation $R_{1 / 3}^{\neq \neq \neq}$is in fact quite simple since it bears a close resemblance to the MONOTONE 1-IN-3-SAT problem. We then use this result in combination with linear variable reductions to relate the problem $\operatorname{SAT}\left(\left\{R_{1 / 3}^{\neq \neq \neq}\right\}\right)$to the exponential-time hypothesis, and prove that $\operatorname{SAT}\left(\left\{R_{1 / 3}^{\neq \neq \neq}\right\}\right)$is solvable in subexponential time if and only if the exponential-time hypothesis is false. This furthermore implies that if there exists a single constraint language $S$ such that SAT $(S)$ is NP-complete and solvable in subexponential time, then the exponential-time hypothesis is false.

In Chapter 8 we continue studying the worst-case time complexity of computational problems, but in the context of the two optimisation problems maximum ones over a finite Boolean constraint language $S$ (MAX-ONES(S)) and the valued constraint satisfaction problem over a finite set of finite-valued cost functions $\Delta(\operatorname{VCSP}(\Delta))$. For the MAX-ONES problem we use essentially the same algebraic techniques that were developed for the CSP problem, and prove that the relation $R_{1 / 3}^{\neq \neq \neq}$resulting in the easiest SAT problem also results in the easiest MAX-ONES problem. The VCSP problem, however, does not follow the standard Galois connection between clones and co-clones, which also means that our approach based on partial clone theory breaks down. Despite this, we show that it is possible to obtain analogous results and give a simple, binary cost function $f$ such that $\operatorname{VCSP}(\{f\})$ results in the easiest NP-complete Boolean VCSP problem. We are then again able to relate the complexity of these problems vis-à-vis the exponential-time hypothesis, and 
for instance prove that if there exists a single constraint language $S$ or a set of cost functions $\Delta$ such that $\operatorname{MAX}-\mathrm{ONES}(S)$ or $\operatorname{VCSP}(\Delta)$ are NP-complete and solvable in subexponential time, then the exponential-time hypothesis is false.

\section{Part IV}

In the concluding part of this thesis we give a brief summary and consider open directions for future research.

\section{Suggested Reading Order}

The two main technical contributions, Part II and Part III, can be read almost independently. The reader with a main interest in computer science is advised to first read Chapter 3 , where the concept of weak bases is introduced, and then read Chapter 7 and Chapter 8 where this is applied to study the computational complexity of constraint satisfaction problems. The reader with a more algebraic interest might instead find Chapter 4 and Chapter 5 to be a more natural continuation. 


\section{Preliminaries}

- These apricots and these make me and to come water in my mouth.

English as she is spoke

In this chapter we introduce the algebraic techniques that will be developed in Part II and applied in Part III of this thesis. We begin in Section 2.1 and Section 2.2 by defining the basic building blocks, functions, relations, and the constraint satisfaction problem. In Section 2.3 and Section 2.4 we then see how these notions can be used to define algebras that will turn out to have many interesting applications from both a practical and theoretical point of view.

\subsection{Functions, Relations and Constraint Languages}

In this thesis we are mainly concerned with functions and relations over a finite domain $D$. We assume that $D \subseteq \mathbb{N}$, although in principle any finite set of values can be used as a domain, and let $\mathrm{OP}_{D}$ denote the set of all (finitary) functions over $D$. We typically use the words operation and function interchangeably in this thesis. Given a function $f: D^{n} \mapsto D, n \in \mathbb{P}$, over $D$ we let $\operatorname{ar}(f)=n$. If $x=\left(x_{1}, \ldots, x_{n}\right) \in D^{n}$ we typically write $f(x)$ to denote the application of $f$ to the arguments $x_{1}, \ldots, x_{n}$.

Similarly, we let $\operatorname{Rel}_{D}$ denote the set of all (finitary) relations over a finite domain $D$. Given a relation $R \subseteq D^{\kappa}, \kappa \in \mathbb{P}$, over a domain $D$ we let $\operatorname{ar}(R)=$ 
$\kappa$. As a convention, we use the Latin symbols $n$ and $m$ to denote arities of functions, and the Greek symbols $\kappa$ and $\lambda$ to denote arities of relations. For a $\kappa$-ary tuple $t$ and $1 \leq i \leq \kappa$, we write $t[i]$ for the $i$ th element of $t$. For $\kappa \geq 1$ and $c \in\{0,1\}$, we write $\vec{c}^{\kappa}$ for the tuple $(c, \ldots, c)$ ( $\kappa$ times), i.e., for a $\kappa$-ary sequence of 0 's or 1 's. If $\kappa$ is clear from the context we simply write $\vec{c}$. A set of relations $S$ is known as a constraint language, or simply language.

It is sometimes convenient to treat relations as predicates and define them in terms of logical formulas over some given constraint language $S$. If $S$ is a constraint language then we use the notation $R\left(x_{1}, \ldots, x_{\kappa}\right) \equiv \phi\left(x_{1}, \ldots, x_{\kappa}\right)$, where $\phi\left(x_{1}, \ldots, x_{k}\right)$ is a first-order formula over $S$ with the free variables $x_{1}, \ldots, x_{\kappa}$, to denote the relation $R=\left\{\left(f\left(x_{1}\right), \ldots, f\left(x_{\kappa}\right)\right) \mid f\right.$ is a model of $\left.\phi\left(x_{1}, \ldots, x_{\kappa}\right)\right\}$.

Example 2.1. Let $R_{1 / 3}=\{(0,0,1),(0,1,0),(1,0,0)\}$. Then the relation $R_{\mathrm{NAE}}=\{(0,0,1),(0,1,0),(1,0,0),(0,1,1),(1,0,1),(1,1,0)\}$ can be defined as $R_{\mathrm{NAE}}\left(x_{1}, x_{2}, x_{3}\right) \equiv \exists x, y \cdot R_{1 / 3}(x, x, y) \wedge\left(R_{1 / 3}\left(x_{1}, x_{2}, x\right) \vee R_{1 / 3}\left(x_{1}, x_{2}, x\right) \vee\right.$ $\left.R_{1 / 3}\left(x_{2}, x_{3}, x\right)\right)$.

When explicitly defining relations, we sometimes use a matrix representation, where the rows of the matrix corresponds to the tuples in the relation. For example, the relation $R_{\mathrm{NAE}}$ in Example 2.1 can be written as

$$
R_{\mathrm{NAE}}=\left(\begin{array}{lll}
0 & 0 & 1 \\
0 & 1 & 0 \\
1 & 0 & 0 \\
0 & 1 & 1 \\
1 & 0 & 1 \\
1 & 1 & 0
\end{array}\right)
$$

Note that the relative order of the columns in the matrix representation does not matter since this only corresponds to a different order of the elements within the tuples.

\subsection{The Constraint Satisfaction Problem}

Let $S$ be a constraint language over a finite domain $D$. The constraint satisfaction problem over $S(\operatorname{CSP}(S))$ is defined as follows.

INSTANCE: A set $V$ of variables and a set $C$ of constraint applications $R\left(v_{1}, \ldots, v_{k}\right)$ where $R \in S, k=\operatorname{ar}(R)$, and $v_{1}, \ldots, v_{k} \in V$.

QUESTION: Is there a total function $f: V \rightarrow D$ such that $\left(f\left(v_{1}\right), \ldots, f\left(v_{k}\right)\right) \in R$ for each constraint $R\left(v_{1}, \ldots, v_{k}\right)$ in $C$ ?

The CSP problem over $S$ can therefore be viewed as the problem of, given a conjunctive formula over $S$, determine whether there exists at least one model of this formula. Hence, whenever convenient, we sometimes treat an 
instance $(V, C)$ of $\operatorname{CSP}(S)$ as a conjunctive formula $\phi$ over $V$ and $S$. In the case where $S$ is a Boolean constraint language we typically denote $\operatorname{CSP}(S)$ as SAT $(S)$. To exemplify, let $R_{\mathrm{NAE}}$ and $R_{1 / 3}$ be defined as in Example 2.1 from Section 2.1. Then the well known NP-complete problem NOT-ALL-EQUAL 3-SAT can be expressed as $\operatorname{SAT}\left(\left\{R_{\mathrm{NAE}}\right\}\right)$. Similarly, $\operatorname{SAT}\left(\left\{R_{1 / 3}\right\}\right)$ can be seen as an alternative formulation of MONOTONE 1-IN-3-SAT.

\subsection{Clone Theory}

Let $F$ be a set of functions over a finite domain. Which other functions can be defined by using functions from F? From Boolean circuit theory we e.g. know that a set of binary NAND gates can be used to implement any other Boolean circuit. In this context one uses the output from one gate and feeds it as input to another gate, in order to describe more advanced circuits. There is a similar notion of composition for functions which allows us to describe complicated functions in terms of simpler functions, which forms the basis of clone theory.

\subsubsection{Clones}

If $f$ is an $n$-ary function over a domain $D$ and $g_{1}, \ldots, g_{n}$ are $m$-ary functions over $D$, then the composition of $f$ and $g_{1}, \ldots, g_{n}$, denoted $f \circ\left(g_{1}, \ldots, g_{n}\right)$, is the $m$-ary function defined by

$$
\left(f \circ\left(g_{1}, \ldots, g_{n}\right)\right)\left(x_{1}, \ldots, x_{m}\right)=f\left(g_{1}\left(x_{1}, \ldots, x_{m}\right), \ldots, g_{n}\left(x_{1}, \ldots, x_{m}\right)\right)
$$

for all $x_{1}, \ldots, x_{m} \in D$.

Example 2.2. Let $f(0)=1, f(1)=0$ and let $g(0,0)=g(0,1)=g(1,0)=$ $0, g(1,1)=1$. Then we see that the binary NAND function can be expressed as $f \circ(g)=f\left(g\left(x_{1}, x_{2}\right)\right)$.

The astute reader will notice that this definition of composition does not allow us to permute the arguments of a function. For instance, given the binary Boolean implication function $i(0,0)=i(0,1)=i(1,1)=1, i(1,0)=0$, then by the above definition of composition we cannot define the function $i^{\prime}(0,0)=i^{\prime}(1,0)=i^{\prime}(1,1)=1, i^{\prime}(0,1)=0$. One way to accomplish this without changing the notion of functional composition is to introduce a set of auxiliary functions whose sole purpose is to return exactly one of its arguments.

Definition 2.3. Let $D$ be a domain. An $n$-ary projection function over $D$ is a function which for some $i \in\{1, \ldots, n\}$ satisfies $\pi_{i}^{n}\left(x_{1}, \ldots, x_{i-1}, x_{i}, x_{i+1}, \ldots, x_{n}\right)=x_{i}$ for all $x_{1}, \ldots, x_{n} \in D$.

Using projection functions it is then easy to see that

$$
i^{\prime}\left(x_{1}, x_{2}\right)=i \circ\left(\pi_{2}^{2}, \pi_{1}^{2}\right)=i\left(\pi_{2}^{2}\left(x_{1}, x_{2}\right), \pi_{1}^{2}\left(x_{1}, x_{2}\right)\right)
$$


since $\pi_{2}^{2}\left(x_{1}, x_{2}\right)=x_{2}$ and $\pi_{1}^{2}\left(x_{1}, x_{2}\right)=x_{1}$ for all $x_{1}, x_{2} \in \mathbb{B}$. We let $\Pi_{D}$ denote the set of all projection functions over a domain $D$. We typically do not explicitly state the domain of a projection function $\pi_{i}^{n}$ since this will always be clear from the context. With this machinery in hand we are now ready to define the concept of clones.

Definition 2.4. Let $D$ be a domain. A set of finitary functions $F$ over $D$ is a clone if $(1) f \circ\left(g_{1}, \ldots, g_{\operatorname{ar}(f)}\right) \in F$ for all $f, g_{1}, \ldots, g_{\operatorname{ar}(f)} \in F$ ( $F$ is closed under functional composition) and (2) $\Pi_{D} \subseteq F$ ( $F$ contains all projection functions).

If $F$ is a set of functions over $D$ then we let $[F]$ denote the smallest clone over $D$ containing $F$. We say that $F$ is a base of $[F]$. A clone is said to be of finite order if it admits a finite base, and is said to be of infinite order otherwise. The operator [.] is a closure operator in the usual mathematical sense, i.e. $F \subseteq[F]$, if $F \subseteq G$ then $[F] \subseteq[G]$, and $[[F]]=[F]$. It is well known that the set of all clones over $D$ form a lattice structure when ordered by set inclusion, where the meet-operator $\sqcap$ is defined as $X \sqcap Y=X \cap Y$ and the join-operator $\sqcup$ as $X \sqcup Y=[X \cup Y]$.

It is readily seen that $\Pi_{D}$ is a clone for every domain $D$ since the composition of two or more projection functions results in a projection function. Moreover, since clones always contain all projection functions, $\Pi_{D}$ is also the smallest clone in the clone lattice over $D$. A natural question to ask is if it is possible to describe the clone lattice over $D$ for any finite domain $D$. Clearly, if $D=\{a\}$ (the domain consists of only one element), then there exists only one clone $\left[\left\{\pi_{1}^{1}\right\}\right]$, where $\pi_{1}^{1}$ is the unary projection function $\pi_{1}^{1}(a)=a$. For $|D|=2$ Post [Pos41] gave a complete classification and proved that this lattice is of countably infinite cardinality. For every $|D| \geq 3$ the lattice is however of uncountably infinite cardinality [YM59].

We close this section by describing Post's result in more detail. When defining Boolean functions we typically use the standard Boolean connectives in infix notation, e.g. $x_{1} \wedge x_{2}$ denotes the binary function $\wedge(0,0)=$ $\wedge(0,1)=\wedge(1,0)=0, \wedge(1,1)=1$, and $\bar{x}=\neg x=1-x$. We occasionally denote the unary complement function $\bar{x}$ by neg $(x)$, i.e. neg $(x)=\bar{x}$. The reader should note that a definition of the form neg $(x)=\bar{x}$ should be read as a shorthand for the slightly more cumbersome statement neg $(x)=\bar{x}$ for all $x \in \mathbb{B}$. Hence, a 'variable' in this context is simply a shorthand for an arbitrary value in the domain. We now introduce some classes of Boolean functions which are necessary in the subsequent treatment, using the notation employed by Reith and Wagner [RW99|, and further propagated in Böhler et al. [BCRV03].

- A Boolean function $f$ is a-reproducing, $a \in\{0,1\}$, if $f(\vec{a})=a$.

- An $n$-ary Boolean function $f$ is monotonic if $f(a) \leq f(b)$ for all $a, b \in$ $\{0,1\}^{n}$ such that $a \leq b$ (componentwise).

- An $n$-ary Boolean function $f$ is self-dual if $f\left(a_{1}, \ldots, a_{n}\right)=$ $\operatorname{dual}(f)\left(a_{1}, \ldots, a_{n}\right)$, where $\operatorname{dual}(f)\left(a_{1}, \ldots, a_{n}\right)=1-f\left(\overline{a_{1}}, \ldots, \overline{a_{n}}\right)$. 
- An $n$-ary Boolean function $f$ is affine if there exists $c_{0}, \ldots, c_{n} \in\{0,1\}$ such that $f\left(a_{1}, \ldots, a_{n}\right)=c_{0} \oplus\left(c_{1} \wedge a_{1}\right) \oplus \ldots \oplus\left(c_{n} \wedge a_{n}\right)$.

- A set $T \subseteq\{0,1\}^{n}$ is $a$-separating, $a \in\{0,1\}$, if there exists $i \in\{1, \ldots, n\}$ such that $a_{i}=a$ for every $\left(a_{1}, \ldots, a_{i}, \ldots, a_{n}\right) \in T$.

- An $n$-ary Boolean function $f$ is $a$-separating, $a \in\{0,1\}$, if $f^{-1}(a)$ is $a$ separating, and it is a-separating of level $k$, if every $T \subseteq f^{-1}(a),|T|=k$, is $a$-separating.

See Table 2.1 for a complete list of all Boolean clones accompanied by a brief description and a base. In this table the constants 0 and 1 are used as shorthands for the unary constant functions $f_{0}(x)=0$ and $f_{1}(x)=1$. The clone consisting of all Boolean functions, $\mathrm{OP}_{\mathbb{B}}$, is denoted by BF. See Figure 2.1 for a visualisation of Post's lattice of Boolean clones.

\subsubsection{Co-Clones}

In Section 2.3.1 we studied closure operators for functions. There is also a similar closure operator for relations which allows us to define an infinite set of relations in terms of a base set of relations. Given a set of relations $S$ we are interested in determining the relations that can be expressed by a certain class of restricted first-order formulas over $S$. Let $\mathrm{Eq}_{D}=\{(x, x) \mid x \in D\}$ denote the equality relation over $D$.

Definition 2.5. Let $S$ be a constraint language over $D$. A $\kappa$-ary relation $R$ has a primitive positive definition (p.p. definition) in $S$ if

$$
R\left(x_{1}, \ldots, x_{\kappa}\right) \equiv \exists y_{1}, \ldots, y_{\mathcal{K}^{\prime}} \cdot R_{1}\left(\mathbf{x}_{\mathbf{1}}\right) \wedge \ldots \wedge R_{m}\left(\mathbf{x}_{\mathbf{m}}\right),
$$

where each $R_{i} \in S \cup\left\{\mathrm{Eq}_{D}\right\}$ and each $\mathbf{x}_{\mathbf{i}}$ is an $\operatorname{ar}\left(R_{i}\right)$-ary tuple of variables over $x_{1}, \ldots, x_{\kappa}, y_{1}, \ldots, y_{\kappa^{\prime}}$.

In other words we allow logical conjunction, existential quantification and equality constraints in these definitions. The reason why p.p. definitions, which are restricted first-order formulas, are preferred over unrestricted firstorder formulas will be made clear in the forthcoming sections, but the reader who anxiously awaits the resolution of this mystery is encouraged to take a quick peek at Theorems 2.11 and 2.13 in Section 2.3.3 
Table 2.1: List of all Boolean clones with definitions and bases, where $h_{n}\left(x_{1}, \ldots, x_{n+1}\right)=\bigvee_{i=1}^{n+1} x_{1} \cdots x_{i-1} x_{i+1} \cdots x_{n+1}$.

\begin{tabular}{|c|c|c|}
\hline Clone & Definition & Base \\
\hline $\mathrm{BF}$ & All Boolean functions & $\{x \wedge y, \neg x\}$ \\
\hline $\mathrm{R}_{0}$ & $\{f \mid f$ is 0 -reproducing $\}$ & $\{x \wedge y, x \oplus y\}$ \\
\hline $\mathrm{R}_{1}$ & $\{f \mid f$ is 1-reproducing $\}$ & $\{x \vee y, x \oplus y \oplus 1\}$ \\
\hline $\mathrm{R}_{2}$ & $\mathrm{R}_{0} \cap \mathrm{R}_{1}$ & $\{x \vee y, x \wedge(y \oplus z \oplus 1)\}$ \\
\hline M & $\{f \mid f$ is monotonic $\}$ & $\{x \vee y, x \wedge y, 0,1\}$ \\
\hline$M_{1}$ & $M \cap R_{1}$ & $\{x \vee y, x \wedge y, 1\}$ \\
\hline$M_{0}$ & $M \cap R_{0}$ & $\{x \vee y, x \wedge y, 0\}$ \\
\hline$M_{2}$ & $M \cap R_{2}$ & $\{x \vee y, x \wedge y\}$ \\
\hline $\mathrm{S}_{0}^{n}$ & $\{f \mid f$ is 0-separating of degree $n\}$ & $\left\{x \rightarrow y\right.$, dual $\left.\left(h_{n}\right)\right\}$ \\
\hline $\mathrm{S}_{0}$ & $\{f \mid f$ is 0 -separating $\}$ & $\{x \rightarrow y\}$ \\
\hline $\mathrm{S}_{1}^{n}$ & $\{f \mid f$ is 1-separating of degree $n\}$ & $\left\{x \wedge \neg y, h_{n}\right\}$ \\
\hline$S_{1}$ & $\{f \mid f$ is 1-separating $\}$ & $\{x \wedge \neg y\}$ \\
\hline $\mathrm{S}_{02}^{n}$ & $\begin{array}{l}S_{0}^{n} \cap R_{2} \\
S_{0} \cap R_{2}\end{array}$ & $\begin{array}{l}\left\{x \vee(y \wedge \neg z), \text { dual }\left(h_{n}\right)\right\} \\
\{x \vee(y \wedge \neg z\}\end{array}$ \\
\hline $\mathrm{S}_{01}^{n}$ & $\mathrm{~S}_{0}^{n} \cap \mathrm{M}$ & $\left\{\operatorname{dual}\left(h_{n}\right), 1\right\}$ \\
\hline $\mathrm{S}_{01}$ & $\mathrm{~S}_{0} \cap \mathrm{M}$ & $\{x \vee(y \wedge z), 1\}$ \\
\hline $\mathrm{S}_{00}^{n}$ & $\mathrm{~S}_{0}^{n} \cap \mathrm{R}_{2} \cap \mathrm{M}$ & $\left\{x \vee(y \wedge z), \operatorname{dual}\left(h_{n}\right)\right\}$ \\
\hline $\mathrm{S}_{00}$ & $\mathrm{~S}_{0} \cap \mathrm{R}_{2} \cap \mathrm{M}$ & $\{x \vee(y \wedge z)\}$ \\
\hline$S_{12}^{n}$ & $\mathrm{~S}_{1}^{n} \cap \mathrm{R}_{2}$ & $\left\{x \wedge(y \vee \neg z), h_{n}\right\}$ \\
\hline $\mathrm{S}_{12}$ & $\mathrm{~S}_{1} \cap \mathrm{R}_{2}$ & $\{x \wedge(y \vee \neg z)\}$ \\
\hline $\mathrm{S}_{11}^{n}$ & $\mathrm{~S}_{1}^{n} \cap \mathrm{M}$ & $\left\{h_{n}, 0\right\}$ \\
\hline$S_{11}^{1+}$ & $\mathrm{S}_{1}^{1} \cap \mathrm{M}$ & $\{x \wedge(y \vee z), 0\}$ \\
\hline$S_{10}^{n}$ & $\mathrm{~S}_{1}^{n} \cap \mathrm{R}_{2} \cap \mathrm{M}$ & $\left\{x \wedge(y \vee z), h_{n}\right\}$ \\
\hline $\mathrm{S}_{10}^{10}$ & $S_{1}^{1} \cap R_{2} \cap M$ & $\{x \wedge(y \vee z)\}$ \\
\hline D & $\{f \mid f$ is self-dual $\}$ & $\{(x \wedge \neg y) \vee(x \wedge \neg z) \vee(\neg y \wedge \neg z)\}$ \\
\hline $\mathrm{D}_{1}$ & $\mathrm{D} \cap \mathrm{R}_{2}$ & $\{(x \wedge y) \vee(x \wedge \neg z) \vee(y \wedge \neg z)\}$ \\
\hline $\mathrm{D}_{2}$ & $D \cap M$ & $\left\{h_{2}\right\}$ \\
\hline L & $\{f \mid f$ is affine $\}$ & $\{x \oplus y, 1\}$ \\
\hline $\mathrm{L}_{0}$ & $\mathrm{~L} \cap \mathrm{R}_{0}$ & $\{x \oplus y\}$ \\
\hline $\mathrm{L}_{1}$ & $L \cap R_{1}$ & $\{x \oplus y \oplus 1\}$ \\
\hline $\mathrm{L}_{2}$ & $L \cap R_{2}$ & $\{x \oplus y \oplus z\}$ \\
\hline $\mathrm{L}_{3}$ & $\mathrm{~L} \cap \mathrm{D}$ & $\{x \oplus y \oplus z \oplus 1\}$ \\
\hline $\mathrm{v}$ & $\{f \mid f$ is a disjunction or constants $\}$ & $\{x \vee y, 0,1\}$ \\
\hline $\mathrm{V}_{0}$ & $\mathrm{~V} \cap \mathrm{R}_{0}$ & $\{x \vee y, 0\}$ \\
\hline $\mathrm{V}_{1}$ & $V \cap R_{1}$ & $\{x \vee y, 1\}$ \\
\hline$v_{2}$ & $\mathrm{~V} \cap \mathrm{R}_{2}$ & $\{x \vee y\}$ \\
\hline $\mathrm{E}$ & $\{f \mid f$ is a conjunction or constants $\}$ & $\{x \wedge y, 0,1\}$ \\
\hline $\mathrm{E}_{0}$ & $\mathrm{E} \cap \mathrm{R}_{0}$ & $\{x \wedge y, 0\}$ \\
\hline $\mathrm{E}_{1}$ & $E \cap R_{1}$ & $\{x \wedge y, 1\}$ \\
\hline $\mathrm{E}_{2}$ & $E \cap R_{2}$ & $\{x \wedge y\}$ \\
\hline $\mathrm{N}$ & $\{f \mid f$ depends on at most one variable $\}$ & $\{\neg x, 0,1\}$ \\
\hline $\mathrm{N}_{2}$ & $\mathrm{~N} \cap \mathrm{R}_{2}$ & $\{\neg x\}$ \\
\hline 1 & $\{f \mid f$ is a projection or a constant $\}$ & $\{0,1\}$ \\
\hline $\mathrm{I}_{0}$ & $I \cap R_{0}$ & $\{0\}$ \\
\hline $\mathrm{I}_{1}$ & $I \cap R_{1}$ & $\{1\}$ \\
\hline $\mathrm{I}_{2}$ & $I \cap R_{2}$ & $\left\{\pi_{1}^{1}\right\}$ \\
\hline
\end{tabular}




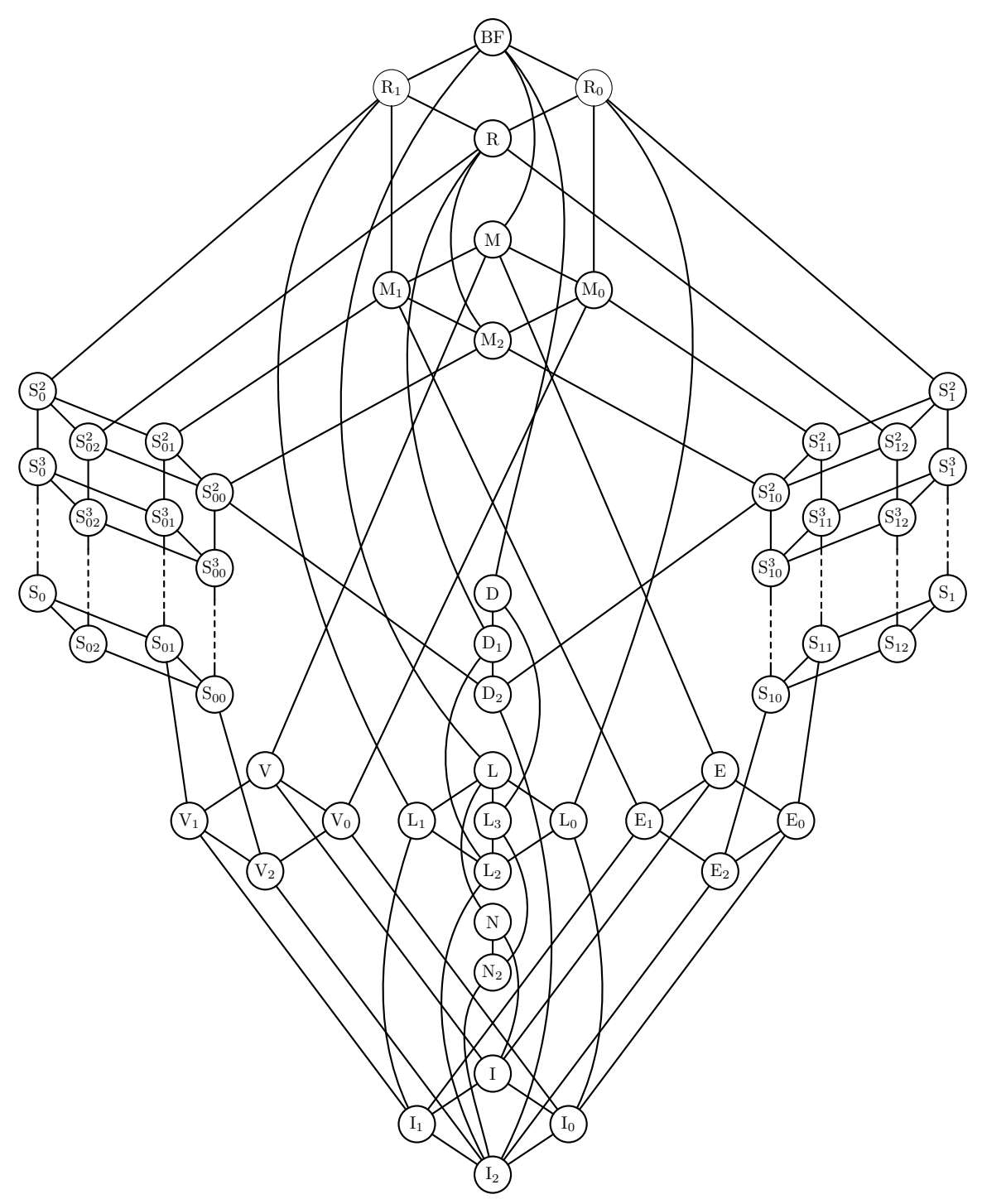

Figure 2.1: The lattice of Boolean clones. 
Example 2.6. Let us return to the relations $R_{\mathrm{NAE}}$ and $R_{1 / 3}$ from Example 2.1 in Section 2.1. We provided the definition $R_{\mathrm{NAE}}\left(x_{1}, x_{2}, x_{3}\right) \equiv \exists x, y \cdot R_{1 / 3}(x, x, y) \wedge$ $\left(R_{1 / 3}\left(x_{1}, x_{2}, x\right) \vee R_{1 / 3}\left(x_{1}, x_{2}, x\right) \vee R_{1 / 3}\left(x_{2}, x_{3}, x\right)\right)$. It is easy to see that this definition is not a p.p. definition since disjunction is used. To see that it is possible to p.p. define $R_{\mathrm{NAE}}$ using $R_{1 / 3}$ we first p.p. define the two auxiliary relations $R_{1}=\{(0,1),(1,0)\}$ and $R_{2}\left(x_{1}, x_{2}, x_{3}\right) \equiv\left(\overline{x_{1}} \vee \overline{x_{2}} \vee \overline{x_{3}}\right)$ as follows:

$$
R_{1}\left(x_{1}, x_{2}\right) \equiv \exists y_{1}, y_{2} \cdot R_{1 / 3}\left(y_{1}, y_{1}, y_{2}\right) \wedge R_{1 / 3}\left(x_{1}, x_{2}, y_{1}\right),
$$

and

$$
\begin{aligned}
R_{2}\left(x_{1}, x_{2}, x_{3}\right) \equiv \exists y_{1}, \ldots, y_{6} \cdot R_{1 / 3}\left(x_{1}, y_{1}, y_{2}\right) & \wedge R_{1 / 3}\left(x_{2}, y_{3}, y_{4}\right) \wedge \\
R_{1 / 3}\left(x_{3}, y_{5}, y_{6}\right) & \wedge R_{1 / 3}\left(y_{1}, y_{3}, y_{5}\right) .
\end{aligned}
$$

And we then see that

$$
\begin{aligned}
R_{\mathrm{NAE}}\left(x_{1}, x_{2}, x_{3}\right) \equiv \exists y_{1}, y_{2}, y_{3} \cdot R_{2}\left(x_{1}, x_{2}, x_{3}\right) \wedge R_{2}\left(y_{1}, y_{2}, y_{3}\right) \wedge \\
R_{1}\left(x_{1}, y_{1}\right) \wedge R_{1}\left(x_{2}, y_{2}\right) \wedge R_{1}\left(x_{3}, y_{3}\right) .
\end{aligned}
$$

What about the other direction? Can $R_{\mathrm{NAE}}$ p.p. define $R_{1 / 3}$ ? Intuitively, it seems feasible since $R_{\mathrm{NAE}}$ might at a first glance appear to be a more complicated and expressive relation than $R_{1 / 3}$. We claim that this is not possible and will give an explicit proof of this in Example 2.12 in Section 2.3.3. The reader who is sceptical to this claim is invited to try to p.p. define $R_{1 / 3}$ using $R_{\mathrm{NAE}}$, but is advised to not spend too much time on this Sisyphean task.

Hence, similar to functional composition in Section 2.3.1. p.p. definitions is our method to obtain more complicated relations from a base set of relations, and we now have everything we need to define the relational analogue of a clone.

Definition 2.7. Let $D$ be a finite domain. A set of relations $S$ over $D$ is a co-clone if (1) $\varnothing \in S$ and (2) $R \in S$ for every $R$ that is p.p. definable over $S$.

Co-clones are also known as relational clones. The reason why we need condition (1) in the above definition is explained in detail in Börner [BÖ8]. If $S$ is a constraint language we similarly to the case of clones let $\langle S\rangle$ denote the smallest co-clone over $D$ containing $S$, and say that $S$ is a base of $\langle S\rangle$. A co-clone is said to be of finite order if it has a finite base and is said to be of infinite order otherwise. It is again easy to verify that $\langle\cdot\rangle$ is indeed a closure operator, and, perhaps not surprisingly, that the co-clones over $D$ with the operators $X \sqcap Y=X \cap Y$ and $X \sqcup Y=\langle X \cup Y\rangle$ forms a lattice. See Figure 2.2 for a visualisation of the Boolean co-clone lattice. In this figure and in the sequel we let $\mathrm{BR}=\operatorname{Rel}_{\mathbb{B}}$ denote the largest Boolean co-clone consisting of all Boolean relations. Co-clones of the form $\left\langle S_{1}\right\rangle \subset\left\langle S_{2}\right\rangle \subset\left\langle S_{3}\right\rangle \subset \ldots$, are sometimes called chains. As evident in Figure 2.2 there are exactly eight chains in the Boolean co-clone lattice. The Argus-eyed reader will probably observe that this lattice is in fact just the Boolean clone lattice turned upside down, and furthermore that the name of each co-clone is of the form IC, 
where $C$ is a clone. This is not due to lack of imagination on the authors part, but because there is a deeper relationship between clones and co-clones, which we investigate in the forthcoming section. Bases for Boolean co-clones were first investigated by Böhler et al. [BSRV05]. We do not include this list since we in the later sections are interested in bases fulfilling some additional properties. We close this section by introducing some simplifying notation for some of the most prominent relations and constraint languages that will be studied in the forthcoming chapters.

- $\mathrm{Neq}_{D}=\{(x, y) \mid x, y \in D, x \neq y\}$,

- if $a_{1}, \ldots, a_{k} \in D$ and $R$ is a $\kappa$-ary relation over $D$ then $R^{a_{1} \ldots a_{k}}=R \times$ $\left\{\left(a_{1}, \ldots, a_{k}\right)\right\}$ is the $(\kappa+k)$-ary relation obtained by augmenting $R$ with the $k$ constant arguments $a_{1}, \ldots, a_{k}$,

- if $R$ is a $\kappa$-ary relation over $D$ then the relation $R^{l \neq}$ (sometimes written $\left.R^{\neq \cdots \neq}\right), l \in\{1, \ldots, \kappa\}$, is the $(\kappa+l)$-ary relation $R^{l \neq}\left(x_{1}, \ldots, x_{\kappa}, x_{\kappa+1}, \ldots, x_{\kappa+l}\right) \equiv R\left(x_{1}, \ldots, x_{\kappa}\right) \wedge \operatorname{Neq}_{D}\left(x_{1}, x_{\kappa+1}\right) \wedge \ldots \wedge$ $\mathrm{Neq}_{D}\left(x_{\kappa}, x_{\kappa+l}\right)$,

- $\mathrm{F}=\{(0)\}$,

- $\mathrm{T}=\{(1)\}$,

- $\mathrm{OR}^{\kappa}\left(x_{1}, \ldots, x_{\kappa}\right) \equiv x_{1} \vee \ldots \vee x_{\kappa}$,

- $\operatorname{NAND}^{\kappa}\left(x_{1}, \ldots, x_{\kappa}\right) \equiv \overline{x_{1}} \vee \ldots \vee \overline{x_{\kappa}}$,

- $\kappa-\mathrm{EVEN}=\left\{\left(x_{1}, \ldots, x_{\kappa}\right) \in \mathbb{B}^{\kappa} \mid \sum_{i=1}^{\kappa} x_{i}\right.$ is even $\}$,

- $\kappa-\mathrm{ODD}=\left\{\left(x_{1}, \ldots, x_{\kappa}\right) \in \mathbb{B}^{\kappa} \mid \sum_{i=1}^{\kappa} x_{i}\right.$ is odd $\}$,

- $\Gamma_{\mathrm{SAT}}^{\kappa}=\left\{\mathbb{B}^{\kappa} \backslash\{t\} \mid t \in \mathbb{B}^{\kappa}\right\}$,

- $\Gamma_{\mathrm{SAT}}=\bigcup_{\mathcal{K}=1}^{\infty} \Gamma_{\mathrm{SAT}}^{\kappa}$,

- $\Gamma_{\mathrm{NAE}}^{\kappa}=\left\{\mathbb{B}^{\kappa} \backslash\{t, \bar{t}\} \mid t \in \mathbb{B}^{\kappa}\right\}$,

- $R_{1 / \kappa}=\left\{\left(x_{1}, \ldots, x_{\kappa}\right) \in \mathbb{B}^{\kappa} \mid \sum_{i=1}^{\kappa} x_{i}=1\right\}$,

We typically write $\mathrm{Neq}$ and $\mathrm{Eq}$ instead of $\mathrm{Neq}_{D}$ and $\mathrm{Eq}_{D}$ since the domain will always be clear from the context. With the help of these relations and the results in Böhler [BSRV05] we e.g. have that

- $\langle\{\mathrm{Eq}\}\rangle=\mathrm{IBF}$,

- $\langle\{F\}\rangle=\mathrm{IR}_{0}$,

- $\langle\{\mathrm{T}\}\rangle=\mathrm{IR}_{1}$,

- $\langle\{\mathrm{Neq}\}\rangle=\mathrm{ID}$, 


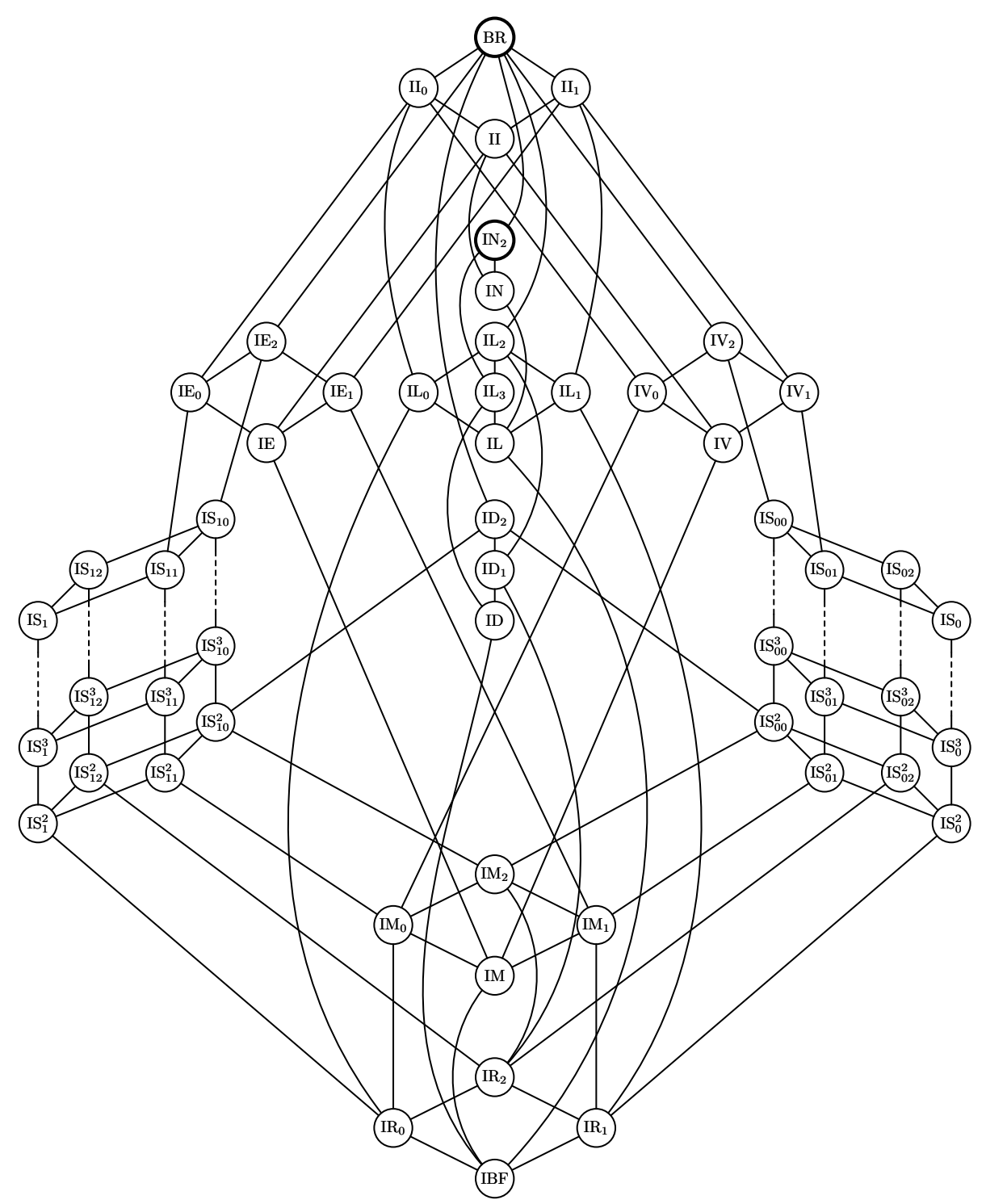

Figure 2.2: The lattice of Boolean co-clones. The co-clones where the SAT $(\cdot)$ problem is NP-hard are drawn in thick black. 
- $\langle\{4-\mathrm{EVEN}\}\rangle=\mathrm{IL}$,

- $\left\langle\left\{\mathrm{OR}^{\kappa}\right\}\right\rangle=\mathrm{IS}_{0}^{\kappa}$ for each $\kappa \geq 2$,

- $\left\langle\left\{\mathrm{NAND}^{\kappa}\right\}\right\rangle=\mathrm{IS}_{1}^{\kappa}$ for each $\kappa \geq 2$,

- $\left\langle\Gamma_{\mathrm{NAE}}^{\kappa}\right\rangle=\mathrm{IN}_{2}$ for each $\kappa \geq 3$, and

- $\left\langle\Gamma_{\text {SAT }}^{\kappa}\right\rangle=\left\langle\left\{R_{1 / \kappa}\right\}\right\rangle=$ BR for each $\kappa \geq 3$.

In certain constraint satisfaction problems it can be convenient to allow complemented arguments. For example, assume that we want to express an $R_{1 / 3}$-constraint where the first variable is complemented. Then it is more convenient to write $R_{1 / 3}\left(\overline{x_{1}}, x_{2}, x_{3}\right)$ rather than $R\left(x_{1}, x_{2}, x_{3}\right)$, where $R=\{(0,0,0),(1,0,1),(1,1,0)\}$. We can model this more formally as follows: let the sign pattern of a constraint $R\left(\ell_{1}, \ldots, \ell_{\kappa}\right)$ be the tuple $\left(s_{1}, \ldots, s_{\kappa}\right)$, where $s_{i}=+$ if $\ell_{i}$ is positive, and $s_{i}=-$ if $\ell_{i}$ is negated. For each sign pattern $\left(s_{1}, \ldots, s_{\kappa}\right)$ we can then associate a relation $R^{\left(s_{1}, \ldots, s_{K}\right)}$ by defining

$$
R^{\left(s_{1}, \ldots, s_{\kappa}\right)}=\left\{\left(x_{1}^{s_{1}}, \ldots, x_{\kappa}^{s_{\kappa}}\right) \mid\left(x_{1}, \ldots, x_{\kappa}\right) \in R\right\},
$$

where $x_{i}^{+}=x_{i}$ and $x_{i}^{-}=\overline{x_{i}}$. For example, $R_{1 / 3}^{(+,-,-)}$denotes the relation $\{(0,1,0),(0,0,1),(1,1,1)\}$ and $R_{1 / 3}^{(-,-,-)}$the relation $\{(1,1,0),(1,0,1),(0,1,1)\}$.

\subsubsection{The Galois Connection between Clones and Co-Clones}

Let us briefly return to the relations $R_{\mathrm{NAE}}$ and $R_{1 / 3}$ from Example 2.1 in Section 2.1. It is not difficult to see that $R_{\mathrm{NAE}}$ is in fact just $R_{1 / 3}$ but with three additional tuples which are the complement of the three first. Hence, if $\left(a_{1}, a_{2}, a_{3}\right) \in R_{\mathrm{NAE}}$ then $\left(\overline{a_{1}}, \overline{a_{2}}, \overline{a_{3}}\right) \in R_{\mathrm{NAE}}$. Another way of viewing this is that if we apply the unary complement function $\bar{x}$ componentwise, to any tuple in $R_{\mathrm{NAE}}$, then the resulting tuple is also included in $R_{\mathrm{NAE}}$. Clearly, this property does not hold for $R_{1 / 3}$, which suggests that there is a difference between the two relations that can be explained by properties of certain functions.

More generally, any $n$-ary function over $D$ can be applied to a sequence of tuples over $D$ as follows: let $t_{1}, \ldots, t_{n}$ be a sequence of $\kappa$-ary tuples over $D$. The $\kappa$-tuple $f\left(t_{1}, \ldots, t_{n}\right)$ is defined as:

$$
\begin{aligned}
f\left(t_{1}, \ldots, t_{n}\right)= & \left(f\left(t_{1}[1], \ldots, t_{n}[1]\right),\right. \\
& \vdots \\
& \left.f\left(t_{1}[\kappa], \ldots, t_{n}[\kappa]\right)\right),
\end{aligned}
$$

Hence, $f$ is applied componentwise to the tuples $t_{1}, \ldots, t_{n}$. We are now ready to define the concept of polymorphisms, which formally captures the earlier intuition that some functions are closely related to the structure of relations. 
Definition 2.8. Let $S$ be a constraint language over $D$ and let $R \in S$. If $f$ is an $n$-ary function over $D$ such that $f\left(t_{1}, \ldots, t_{n}\right) \in R$ for all $t_{1}, \ldots, t_{n} \in R$, then $R$ is said to be closed (or invariant) under $f$. If all relations in $S$ are closed under $f$ then $S$ is said to be closed under $f$. A function $f$ such that $S$ is closed under $f$ is called a polymorphism of $S$. The set of all polymorphisms over $D$ of $S$ is denoted by $\operatorname{Pol}_{D}(S)$. Given a set of functions $F$, the set of all relations over $D$ that are invariant under all functions in $F$ is denoted by $\operatorname{Inv}_{D}(F)$.

If the domain is clear from the context then we usually write $\operatorname{Pol}(S)$ and $\operatorname{Inv}(F)$ instead of $\operatorname{Pol}_{D}(S)$ and $\operatorname{Inv}_{D}(F)$. To save a few additional keystrokes we sporadically write IC instead of $\operatorname{Inv}(C)$, and implicitly assume that $C$ is a clone. With these notions we can now say that neg $\in \operatorname{Pol}\left(\left\{R_{\mathrm{NAE}}\right\}\right)$ and that neg $\notin \operatorname{Pol}\left(\left\{R_{1 / 3}\right\}\right)$. Hence, neg is a polymorphism of $R_{\mathrm{NAE}}$ but does not preserve $R_{1 / 3}$.

Example 2.9. The ternary majority function $f$ over the Boolean domain is the (unique) function satisfying $f(a, a, b)=f(a, b, a)=f(b, a, a)=a$ for all $a, b \in\{0,1\}$. Let $\mathrm{R}=\{(0,0,1),(1,0,0),(0,1,1),(1,0,1)\}$. It is then easy to verify that $f\left(t_{1}, t_{2}, t_{3}\right) \in R$ for every triple of tuples, $t_{1}, t_{2}, t_{3} \in R$. For example, if $t_{1}=(0,0,1), t_{2}=(0,1,1)$ and $t_{3}=(1,0,1)$, then

$$
\begin{aligned}
f\left(t_{1}, t_{2}, t_{3}\right) & =\left(f\left(t_{1}[1], t_{2}[1], t_{3}[1]\right), f\left(t_{1}[2], t_{2}[2], t_{3}[2]\right), f\left(t_{1}[3], t_{2}[3], t_{3}[3]\right)\right) \\
& =(f(0,0,1), f(0,1,0), f(1,1,1)) \\
& =(0,0,1) \in R .
\end{aligned}
$$

We conclude that $R$ is invariant under $f$ or, equivalently, that $f$ is a polymorphism of $R$. In contrast, if $g$ is the ternary affine function over the Boolean domain, defined by $g(x, y, z)=x \oplus y \oplus z$, then

$$
\begin{aligned}
g\left(t_{1}, t_{2}, t_{3}\right) & =\left(g\left(t_{1}[1], t_{2}[1], t_{3}[1]\right), g\left(t_{1}[2], t_{2}[2], t_{3}[2]\right), g\left(t_{1}[3], t_{2}[3], t_{3}[3]\right)\right) \\
& =(g(0,0,1), g(0,1,0), g(1,1,1)) \\
& =(1,1,1) \notin R .
\end{aligned}
$$

Hence, $g$ is not a polymorphism of $R$.

What can be said of the structure of $\operatorname{Pol}(S)$ ? First, observe that $\Pi_{D} \subseteq$ $\operatorname{Pol}_{D}(S)$ since a projection function trivially preserves all relations. Second, note that if $f, g_{1}, \ldots, g_{m} \in \operatorname{Pol}(S)$ then the composition of $f$ and $g_{1}, \ldots, g_{m}$ will be included in $\operatorname{Pol}(S)$. Hence, sets of the form $\operatorname{Pol}(S)$ are in fact nothing more than clones in disguise, defined in terms of relations instead of functions. Similarly, one can show that if $S^{\prime} \subseteq \operatorname{Inv}(F)$ and if $R$ is p.p. definable over $S^{\prime}$, then $R \in \operatorname{Inv}(F)$, which shows that sets of the form $\operatorname{Inv}(F)$ are coclones defined in terms of functions. We thus obtain the following theorem.

Theorem 2.10. [BKKR69a: BKKR69b: Gei68] Let $S$ be a constraint language over a finite domain $D$ and let $F$ be a set of functions over $D$. Then 
- $\langle S\rangle=\operatorname{Inv}(\operatorname{Pol}(S))$,

- $[F]=\operatorname{Pol}(\operatorname{Inv}(F))$.

Now we are finally in a position to explicate the relationship between clones and co-clones with the help of the $\operatorname{Inv}(\cdot)$ and $\operatorname{Pol}(\cdot)$ operators.

Theorem 2.11. BKKR69a: BKKR69b: Gei68] Let $S$ and $S^{\prime}$ be constraint languages over a finite domain. Then $S \subseteq\left\langle S^{\prime}\right\rangle$ if and only if $\operatorname{Pol}\left(S^{\prime}\right) \subseteq \operatorname{Pol}(S)$.

This inverse relationship is in general known as a Galois connection. The theorem is very useful since we now have two ways to approach a problem, as the following example highlights.

Example 2.12. Recall that we in Example 2.6 proved that $\left\langle\left\{R_{\mathrm{NAE}}\right\}\right\rangle \subseteq$ $\left\langle\left\{R_{1 / 3}\right\}\right\rangle$ by providing a quite complicated p.p. definition of $R_{\mathrm{NAE}}$ over $R_{1 / 3}$. There is now a much simpler way to prove this by exploiting the Galois connection. First, with the help of Post's lattice one can easily verify that $\operatorname{Pol}\left(\left\{R_{\mathrm{NAE}}\right\}\right)=[\{n e g\}]=\mathrm{N}_{2}$ and that $\operatorname{Pol}\left(\left\{R_{1 / 3}\right\}\right)=\left[\left\{\pi_{1}^{1}\right\}\right]=\mathrm{I}_{2}$. This implies that $\operatorname{Pol}\left(\left\{R_{1 / 3}\right\}\right) \subset \operatorname{Pol}\left(\left\{R_{\mathrm{NAE}}\right\}\right)$ and according to the Galois connection that $\left\langle\left\{R_{\mathrm{NAE}}\right\}\right\rangle \subset\left\langle\left\{R_{1 / 3}\right\}\right\rangle$. On the other hand, since $\left\langle\left\{R_{\mathrm{NAE}}\right\}\right\rangle \subset\left\langle\left\{R_{1 / 3}\right\}\right\rangle$, it follows that $R_{1 / 3} \notin\left\langle\left\{R_{\mathrm{NAE}}\right\}\right\rangle$. Hence, in this example, we could immediately verify that $R_{1 / 3}$ can p.p. define $R_{\mathrm{NAE}}$ simply by comparing the polymorphisms of the two constraint languages. Similarly, we could also prove that $R_{\mathrm{NAE}}$ cannot p.p. define $R_{1 / 3}$; a fact that is not obvious at all when working on the relational side with p.p. definitions.

The following result forms the basis of the algebraic approach for analysing the complexity of $\operatorname{SAT}(\cdot)$ and $\operatorname{CSP}(\cdot)$. It states that the complexity of $\operatorname{CSP}(S)$ is determined, up to polynomial-time reductions, by the polymorphisms of S.

Theorem 2.13 (Jeavons [Jea98]). Let $S$ and $S^{\prime}$ be finite constraint languages over a finite domain $D$. If $\operatorname{Pol}\left(S^{\prime}\right) \subseteq \operatorname{Pol}(S)$, then $\operatorname{CSP}(S)$ is polynomial-time many-one reducible to $\operatorname{CSP}\left(S^{\prime}\right)$.

Using the Galois connection in Theorem 2.11, it is not difficult to see that Theorem 2.13 holds, since we can replace every constraint in an instance of $\operatorname{CSP}(S)$ by its p.p. definition over $S^{\prime}$, identify all variables occurring in equality constraints, and remove all existential quantifiers. This can be done in polynomial time since both languages are finite. Nevertheless, this is a very powerful theorem, and it is worth noting that Schaefer's complexity classification of SAT $(S)$ [Sch78] follows more or less directly from this result together with Post's lattice of clones. In our terminology Schaefer's theorem can now be restated as: $\operatorname{SAT}(S)$ is NP-complete if and only if $\operatorname{Pol}(S)=\operatorname{Pol}\left(\left\{R_{1 / 3}\right\}\right)=\mathrm{I}_{2}$ or $\operatorname{Pol}(S)=\operatorname{Pol}\left(\left\{R_{\mathrm{NAE}}\right\}\right)=\mathrm{N}_{2}$. Hence, out of the countably infinite number of Boolean clones, only two of these correspond to NP-complete SAT $(\cdot)$ problems. 


\subsection{Partial Clone Theory}

So far we have studied two closure operators for functions and relations and showed that there is a powerful connection between them. Clone theory has had remarkable success in providing complexity classifications for problems parameterized by constraint languages, cf. the survey by Creignou et al. |CV08|. Unfortunately, we cannot use Theorem 2.13 to reason about the worst-case time complexity of NP-complete $\operatorname{CSP}(\cdot)$ problems, since even if $\operatorname{Pol}(S)=\operatorname{Pol}\left(S^{\prime}\right)$, the two problems $\operatorname{CSP}(S)$ and $\operatorname{CSP}\left(S^{\prime}\right)$ might differ wildly in worst-case time complexity. To see this, note that $\operatorname{Pol}\left(\Gamma_{\mathrm{SAT}}\right)=$ $\operatorname{Pol}\left(\left\{R_{1 / 3}\right\}\right)=\mathrm{I}_{2}$, even though $\operatorname{SAT}\left(\left\{R_{1 / 3}\right\}\right)$ is known to be solvable in $O\left(1.0984^{|V|}\right)$ time, while $\operatorname{SAT}\left(\Gamma_{\mathrm{SAT}}\right)$ is not known to be solvable strictly faster than $O\left(2^{|V|}\right)$ time. In other words, the lattice of clones is not fine grained enough to compare the worst-case time complexity of NP-complete $\operatorname{CSP}(\cdot)$ problems, and constraint languages that apparently have very different computational properties can still have the same polymorphisms. Hence, we want to find an alternative notion of polymorphisms, so that languages such as $\Gamma_{\mathrm{SAT}}$ and $\left\{R_{1 / 3}\right\}$ correspond to different clones. One way to get a more refined framework is to consider partial functions in Definition 2.8. which we investigate in the forthcoming sections.

\subsubsection{Clones of Partial Functions}

An $n$-ary partial function $f$ on a finite domain $D$ is a map $f: X \rightarrow D$ where $X \subseteq D^{n}$. In other words, $f$ is a function that is allowed to be undefined for some sequences of arguments. For a partial function $f: X \rightarrow D$, $X \subseteq D^{n}$, we let domain $(f)=X$ and $\operatorname{ar}(f)=n$. Note that we do not require that domain $(f)$ is a proper subset of $D^{\operatorname{ar}(f)}$. A partial function $g$ is said to be a subfunction of a partial function $f$ if domain $(g) \subseteq \operatorname{domain}(f)$ and $g\left(x_{1}, \ldots, x_{n}\right)=f\left(x_{1}, \ldots x_{n}\right)$ for all $\left(x_{1}, \ldots, x_{n}\right) \in \operatorname{domain}(g)$. We also say that $g$ is $f$ restricted to $X$. Finally, a set of partial functions $F$ is said to be strong if for every partial function $f \in F$ and every subfunction $g$ of $f$, $g$ is also included in F. A partial projection function is a subfunction of a total projection function, and we let $\Pi_{D}^{p}$ denote the set of all partial projection functions over the domain $D$.

Compositon of partial functions can be defined in an analogous manner to composition of total functions. If $f$ is an $n$-ary partial function over $D$ and $g_{1}, \ldots, g_{n}$ are $m$-ary partial functions over $D$, then the composition of $f$ and $g_{1}, \ldots, g_{n}$, denoted $f \circ\left(g_{1}, \ldots, g_{n}\right)$, is the $m$-ary partial function defined by

$$
\left(f \circ\left(g_{1}, \ldots, g_{n}\right)\right)(\mathbf{x})=f\left(g_{1}(\mathbf{x}), \ldots, g_{n}(\mathbf{x})\right)
$$

and

$$
\begin{aligned}
\operatorname{domain}\left(f \circ\left(g_{1}, \ldots, g_{n}\right)\right)= & \left\{\mathbf{x} \in \bigcap_{i=1}^{n} \operatorname{domain}\left(g_{i}\right) \mid\right. \\
& \left.\left(g_{1}(\mathbf{x}), \ldots, g_{n}(\mathbf{x})\right) \in \operatorname{domain}(f)\right\},
\end{aligned}
$$


where $\mathbf{x}=\left(x_{1}, \ldots, x_{m}\right)$. Even though the latter part of this definition might appear rather complicated, it only states that the composed function is defined if all involved function applications are defined. We are now ready to define the concept of a strong partial clone.

Definition 2.14. Let $D$ be a finite domain. A set of partial functions $F$ over $D$ is a strong partial clone if $(1) f \circ\left(g_{1}, \ldots, g_{\operatorname{ar}(f)}\right) \in F$ for all $f, g_{1}, \ldots, g_{\operatorname{ar}(f)} \in F(F$ is closed under composition of partial functions) and (2) $\Pi_{D}^{p} \subseteq F$ (F contains all partial projection functions).

Given a set $F$ of partial functions over $D$ we let $[F]_{S}$ denote the smallest strong partial clone over $D$ containing $F$. Similar to the case of clones the set $F$ is said to be a base of $[F]_{S}$, and a strong partial clone is of finite order if it has a finite base and is said to be of infinite order otherwise. It is not difficult to see that the condition $\Pi_{D}^{p} \subseteq F$ implies that if $f \in F$ then $F$ also contains all subfunctions of $F$, hence the term strong. We remark that if the second condition in Definition 2.14 is changed to $\Pi_{D} \subseteq F$ then one obtains a partial clone instead of a strong partial clone, i.e., a composition-closed set of partial functions, but which does not necessarily include all subfunctions. However, in this thesis we will only be concerned with strong partial clones, since they as evident in Section 2.4.2, admit simple relational descriptions.

The reader might now ask why we do not include a picture akin to Figure 2.1 to show the inclusion structure of the strong partial Boolean clones. There is a simple reason for this: the lattice of strong partial clones is uncountably infinite even for the Boolean domain [AV94]. Hence, the subtle step from total to partial functions is not so simple that one might believe at a first glance. Of course, a priori, the cardinality of a mathematical structure does not really say anything about how useful it is, and we remark that uncountably infinite lattices have proven to be remarkably useful when studying the complexity of infinite-domain constraint satisfaction problems |Bod12|.

\subsubsection{Weak Partial Co-Clones}

It might not be very obvious why strong partial clones are at all interesting from a complexity theoretical point of view. The relational point of view, which we investigate in this section, will hopefully make this clearer.

Definition 2.15. Let $S$ be a constraint language over $D$. A $\kappa$-ary relation $R$ has a quantifier-free primitive positive definition (q.f.p.p. definition) in $S$ if

$$
R\left(x_{1}, \ldots, x_{\kappa}\right) \equiv R_{1}\left(\mathbf{x}_{\mathbf{1}}\right) \wedge \ldots \wedge R_{m}\left(\mathbf{x}_{\mathbf{m}}\right),
$$

where each $R_{i} \in S \cup\{\mathrm{Eq}\}$ and each $\mathbf{x}_{\mathbf{i}}$ is an $\operatorname{ar}\left(R_{i}\right)$-ary tuple of variables over $x_{1}, \ldots, x_{\kappa}$.

As the name suggests, the only difference compared to p.p. definitions is that we do not allow existential quantification. 
Definition 2.16. Let $D$ be a domain. A set of finitary relations $S$ over $D$ is a weak partial co-clone if (1) $\varnothing \in S$ and (2) $R \in S$ for every $R$ that is q.f.p.p. definable over $S$.

The term weak system and partial co-clone have also been used for these sets |BHP91; Lau06|. Similar to the case of co-clones we let $\langle S\rangle_{\exists}$ denote the smallest weak partial co-clone containing $S$. The set $S$ is again called a base of $\langle S\rangle_{\exists}$, and a weak partial co-clone is said to be of finite order if it has a finite base and is of infinite order otherwise.

Example 2.17. Let us consider the expressive power of $R_{1 / 3}$ compared to $\Gamma_{\text {SAT }}^{3}$ with respect to q.f.p.p. definitions, i.e., does it hold that $\langle\{R\}\rangle_{\nexists}=\left\langle\Gamma_{\mathrm{SAT}}^{3}\right\rangle_{\nexists \prime}$, that $\langle\{R\}\rangle_{\exists} \subset\left\langle\Gamma_{\mathrm{SAT}}^{3}\right\rangle_{\nexists}$, or that $\left\langle\Gamma_{\text {SAT }}^{3}\right\rangle_{\exists} \subset\left\langle\left\{R_{1 / 3}\right\}\right\rangle_{\exists}$ ? It is not so difficult to see that $\Gamma_{\mathrm{SAT}}^{3}$ is a rather expressive language since we can q.f.p.p. define any ternary relation $R$ by representing each $t \in \mathbb{B}^{3} \backslash R$ by a 3-clause. In the case of $R_{1 / 3}$ note that

$$
\mathbb{B}^{3} \backslash R_{1 / 3}=\{(0,0,0),(0,1,1),(1,0,1),(1,1,0),(1,1,1)\},
$$

which implies that

$$
\begin{aligned}
R_{1 / 3}\left(x_{1}, x_{2}, x_{3}\right) \equiv & \left(x_{1} \vee x_{2} \vee x_{3}\right) \wedge\left(x_{1} \vee \overline{x_{2}} \vee \overline{x_{3}}\right) \wedge\left(\overline{x_{1}} \vee x_{2} \vee \overline{x_{3}}\right) \wedge \\
& \left(\overline{x_{1}} \vee \overline{x_{2}} \vee x_{3}\right) \wedge\left(\overline{x_{1}} \vee \overline{x_{2}} \vee \overline{x_{3}}\right),
\end{aligned}
$$

from which it follows that $R_{1 / 3} \in\left\langle\Gamma_{\mathrm{SAT}}^{3}\right\rangle_{\nexists \prime}$, and hence that $\left\langle\left\{R_{1 / 3}\right\}\right\rangle_{\exists} \subseteq\left\langle\Gamma_{\mathrm{SAT}}^{3}\right\rangle_{\nexists}$. Could it also be the case that $\Gamma_{\text {SAT }}^{3} \subseteq\left\langle\left\{R_{1 / 3}\right\}\right\rangle_{\nexists}$ ? This mystery will be resolved in Example 2.22 in Section 2.4.3

\subsubsection{The Galois Connection between Strong Partial Clones and Weak Partial Co-Clones}

We now want to obtain a corresponding Galois connection similar to that of Theorem 2.11 between strong partial clones and weak partial co-clones. Hence, we need a new notion of polymorphisms.

Definition 2.18. Let $S$ be a constraint language over $D$ and let $R \in S$. If $f$ is an $n$-ary partial function over $D$ such that for all $t_{1}, \ldots, t_{n}$ either (1) $f\left(t_{1}, \ldots, t_{n}\right) \in R$ or (2) $f\left(t_{1}, \ldots, t_{n}\right)$ is undefined, then $R$ is said to be closed (or invariant) under $f$. If all relations in $S$ are closed under $f$ then $S$ is said to be closed under $f$. A partial function $f$ such that $S$ is closed under $f$ is called a partial polymorphism of $S$. The set of all partial polymorphisms over $D$ of $S$ is denoted by $\mathrm{pPol}_{D}(S)$. Given a set of partial functions $F$, the set of all relations over $D$ that invariant under all functions in $F$ is denoted by $\operatorname{Inv}_{D}(F)$.

Example 2.19. Consider again the relation $R$ and the affine function $g$ from Example 2.9 and let $p$ be the partial function defined as $p(x, y, z)=g(x, y, z)$ except that it is undefined for $(1,1,0),(1,0,1),(0,1,1)$ and $(1,1,1)$. Now it can be verified that $p$ is a partial polymorphism of $R$. 
As usual, we write $\operatorname{Inv}(F)$ and $\mathrm{pPol}(S)$ instead of $\operatorname{Inv}_{D}(F)$ and $\mathrm{pPol}_{D}(S)$ whenever the domain is clear from the context. It is again not so difficult to verify that sets of the form $\mathrm{pPol}(S)$ are strong partial clones while sets of the form $\operatorname{Inv}(F)$, where $F$ is a set of partial functions, are weak partial co-clones.

Theorem 2.20. Gei68: Rom81] Let $S$ be a constraint language over a finite domain $D$ and let $F$ be a set of partial functions over $D$. Then

- $\langle S\rangle_{\exists}=\operatorname{Inv}(\operatorname{pPol}(S))$,

- $[F]_{s}=\operatorname{pPol}(\operatorname{Inv}(F))$.

We then have the following Galois connection between strong partial clones and weak partial co-clones, first proved by Geiger[Gei68] and later studied in greater detail by Romov |Rom81|.

Theorem 2.21. Gei68, Rom81 Let $S$ and $S^{\prime}$ be constraint languages. Then $S \subseteq$ $\left\langle S^{\prime}\right\rangle_{\exists}$ if and only if $\mathrm{pPol}\left(S^{\prime}\right) \subseteq \operatorname{pPol}(S)$.

It might not be very obvious why the Galois connection in Theorem 2.21 actually holds. Recall that we started with defining the concept of a strong partial clone. Note first that a strong partial clone is richer than a clone in the sense that $\mathrm{pPol}(S) \supseteq \operatorname{Pol}(S)$ always holds. Similarly, a weak partial co-clone is a simpler mathematical object than a co-clone in the sense that $\langle S\rangle_{\exists} \subseteq\langle S\rangle$ also always holds. The intuition is then, due to the inverse nature in a Galois connection, that a complicated set of functions has to correspond to a simple set of relations. Hence, we had to find a closure operator based on logical formulas strictly weaker than p.p. definitions, which we did by eliminating the usage of existential quantifiers.

Example 2.22. Let us return to $\left\langle\left\{R_{1 / 3}\right\}\right\rangle_{\exists}$ and $\left\langle\Gamma_{\text {SAT }}^{3}\right\rangle_{\exists}$ in Example 2.17 We left the question of whether $\Gamma_{\text {SAT }}^{3} \subseteq\left\langle\left\{R_{1 / 3}\right\}\right\rangle_{\exists}$ dangling in the air. Using the Galois connection in Theorem 2.21 we are now in a position to prove that $\Gamma_{\text {SAT }}^{3} \not \subset$

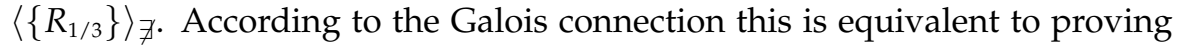
that $\mathrm{pPol}\left(\Gamma_{\mathrm{SAT}}^{3}\right) \subset \operatorname{pPol}\left(\left\{R_{1 / 3}\right\}\right)$, i.e., that there exists a partial polymorphism of $R_{1 / 3}$ that does not preserve $\Gamma_{\mathrm{SAT}}^{3}$. Define the binary partial function $f$ as $f(0,1)=f(1,0)=f(1,1)$ and undefined for $(0,0)$. Then it is not so difficult to verify that $f \in \operatorname{pPol}\left(\left\{R_{1 / 3}\right\}\right)$ since, for any two tuples $t_{1}, t_{2} \in R_{1 / 3}, f\left(t_{1}, t_{2}\right)$ is undefined. For example, $f((0,0,1),(0,1,0))=(f(0,0), f(0,1), f(1,0))$ is undefined since $f(0,0)$ is undefined.

Now, to prove that $f \notin \operatorname{pPol}\left(\Gamma_{\mathrm{SAT}}^{3}\right)$ we have to find a relation $R \in \Gamma_{\mathrm{SAT}}^{3}$ and $t_{1}, t_{2} \in R$ such that $f\left(t_{1}, t_{2}\right) \notin R$. If we e.g. choose $R\left(x_{1}, x_{2}, x_{3}\right) \equiv\left(x_{1} \vee\right.$ $\left.x_{2} \vee x_{3}\right)$ and the two tuples $(0,1,1),(1,1,1) \in R$ then $f((0,1,1),(1,1,1))=$ $(f(0,1), f(1,1), f(1,1))=(0,0,0) \notin R$. Hence, $f \notin \operatorname{pPol}\left(\Gamma_{\mathrm{SAT}}^{3}\right)$, from which it follows that $\operatorname{pPol}\left(\Gamma_{\text {SAT }}^{3}\right) \subset \operatorname{pPol}\left(\left\{R_{1 / 3}\right\}\right)$ and, due to the Galois connection, that $\left\langle\left\{R_{1 / 3}\right\}\right\rangle_{\nexists} \subset\left\langle\Gamma_{\text {SAT }}^{3}\right\rangle_{\nexists}$.

The example above illustrates that we have succeeded in our goal of finding a more fine-grained clone lattice, since $\operatorname{Pol}\left(\left\{R_{1 / 3}\right\}\right)=\operatorname{Pol}\left(\Gamma_{\mathrm{SAT}}^{3}\right)$. 
Moreover, it is interesting to note that the complexity of $\operatorname{SAT}\left(\left\{R_{1 / 3}\right\}\right)$ and $\operatorname{SAT}\left(\Gamma_{\mathrm{SAT}}^{3}\right)$ seems to differ in practice [Her14; Wah07], which suggests that the partial polymorphisms of a constraint language $S$ have a relationship with the complexity of SAT $(S)$. We return to this question in Part III of this thesis where we study the complexity of $\mathrm{SAT}(\cdot)$ and several related optimisation problems, using the theory developed in Part II 


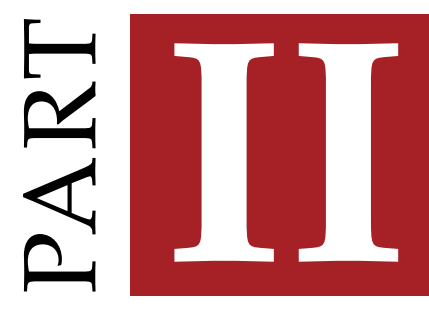

Properties of Strong Partial Clones 



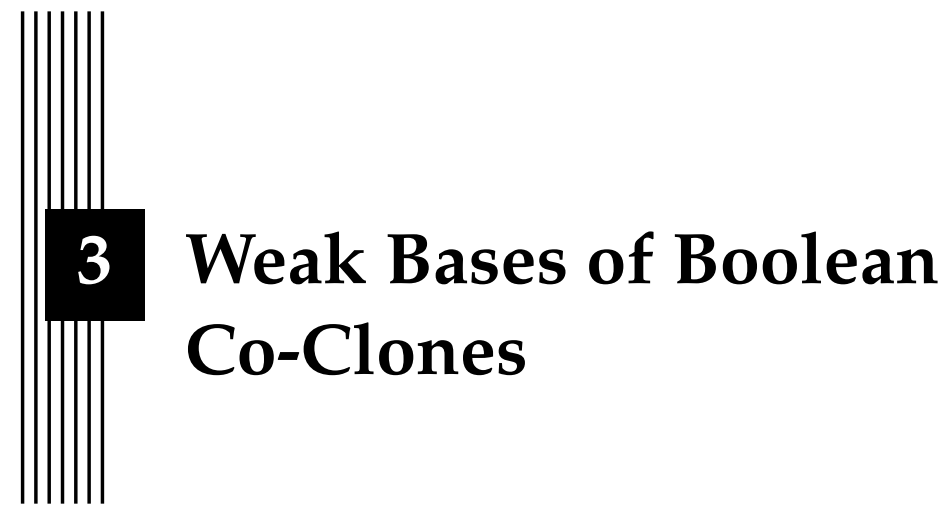

Diseases.

The megrime. The whitlow. The rheumatisme. The vomitory.

English as she is spoke

In this chapter we investigate constraint languages known as weak bases [SS08|. Intuitively, a weak base of a co-clone is a base that have a larger number of partial polymorphisms than any other base of the co-clone. As we will see throughout this thesis, weak bases have many interesting properties, which makes them highly usable from both a theoretical and practical view point.

\subsection{Introduction}

As mentioned in Chapter 2, the lattice of strong partial clones is of uncountable infinite cardinality even for the Boolean domain. Hence, even though Example 2.22 suggests that there is a relationship between the partial polymorphisms of a finite constraint language $S$ and the complexity of the corresponding $\operatorname{CSP}(S)$ problem, this observation is of little use if we have no understanding of the strong partial clone lattice. Let us instead consider a slightly more manageable strategy, where we restrict our focus to certain parts of this lattice. To take a concrete example, take the relation $R_{1 / 3}=$ $\{(0,0,1),(0,1,0),(1,0,0)\}$ from Chapter 2. and recall that $\operatorname{CSP}\left(\left\{R_{1 / 3}\right\}\right)$ is $\mathrm{NP}$-complete. Is it possible to characterise all strong partial clones $\mathrm{pPol}(S)$ 
such that $\operatorname{Pol}(S)=\operatorname{Pol}\left(\left\{R_{1 / 3}\right\}\right)$ ? Or is it at least possible to say anything about the strong partial clones $\operatorname{pPol}(S)$ such that $\operatorname{Pol}(S)=\operatorname{Pol}\left(\left\{R_{1 / 3}\right\}\right)$ and $\operatorname{pPol}(S) \supset \operatorname{pPol}\left(\left\{R_{1 / 3}\right\}\right)$ ? In the context of constraint satisfaction problem, these questions were first raised by Schnoor and Schnoor [SS08], who proved that for any clone $\mathrm{C}$ of finite order, the set of all strong partial clones $\mathrm{pPol}(S)$ such that $\operatorname{Pol}(S)=\mathrm{C}$, has a uniquely determined largest element. Relational descriptions of these largest elements are known as weak bases [SS08]. In this chapter we build upon this result and provide weak bases for all Boolean coclones. The main theorem is proved in Section 3.3.1 and Section 3.3.2 where we provide weak bases of Boolean co-clones of finite and infinite order, respectively. Our results differ from Schnoor and Schnoor [SS08; Sch08] in three important aspects. First, each weak base that we determine can in a natural sense be considered to be minimal. Second, we present alternative proofs where Schnoor's and Schnoor's procedure results in relations which are exponentially larger than the bases given by Böhler et al. [BSRV05] and Creignou et al.|CKZ08|. Third, we extend the notion of a weak base of a co-clone $\operatorname{Inv}(\mathrm{C})$ to also cover the case where $\operatorname{Inv}(\mathrm{C})$ is of infinite order.

\subsection{Preliminaries}

We first define the concept of an interval of strong partial clones corresponding to a total clone. Intuitively, given a clone $C$, this is the set of all strong partial clones whose total component equals $\mathrm{C}$.

Definition 3.1. Let $C$ be a clone over a finite domain $D$. The interval of $C$, $\operatorname{Int}_{[\cdot]_{s}}(\mathrm{C})$, is the set $\operatorname{Int}_{[\cdot]_{s}}(\mathrm{C})=\left\{\operatorname{pPol}(S) \mid S \subseteq \operatorname{Rel}_{D}, \operatorname{Pol}(S)=\mathrm{C}\right\}$.

Given a Boolean clone $\mathrm{C}$ it is now natural to try to determine the cardinality of $\operatorname{Int}_{[\cdot]_{s}}(\mathrm{C})$. Since the lattice of Boolean strong partial clones is of uncountably infinite cardinality, and the lattice of Boolean clones is of countably infinite cardinality, it follows that the cardinality of $\operatorname{Int}_{[\cdot]_{s}}(\mathrm{C})$ in general can be equal to the continuum. More surprising is the fact that for Boolean clones intervals of this form are always either finite or uncountably infinite [Sch15]. In particular, assuming $\mathrm{P} \neq \mathrm{NP}$, $\operatorname{Int}_{\cdot \cdot]_{s}}(\operatorname{Pol}(S))$ is of uncountably infinite cardinality whenever CSP $(S)$ is NP-complete. Hence, we cannot hope to completely describe these intervals even for the restricted case when $\operatorname{Pol}(S) \subseteq \mathrm{N}_{2}$. Nevertheless, we can provide some insight into the structures of these intervals by using the concept of a weak base.

Definition 3.2. Let $C$ be a clone. A constraint language $S$ is a weak base of

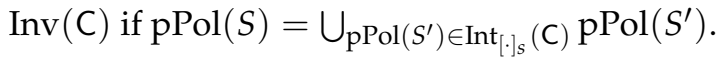

Although perhaps not very obvious from the definition, a weak base $S$ is also a base of $\operatorname{Inv}(\mathrm{C})$ since $\operatorname{pPol}(S) \in \operatorname{Int}_{[\cdot]_{S}}(\mathrm{C})[\overline{\mathrm{SS} 08} \mid$. Now, observe that since $\mathrm{pPol}(S)$ is defined to be the union of all strong partial clones in $\operatorname{Int}_{[\cdot]_{s}}(\mathrm{C}), \operatorname{pPol}(S)$ is also the largest element in $\operatorname{Int}_{[\cdot]_{s}}(\mathrm{C})$, which implies that $\operatorname{pPol}(S) \supseteq \operatorname{pPol}\left(S^{\prime}\right)$ for any $\operatorname{pPol}\left(S^{\prime}\right) \in \operatorname{Int}_{[\cdot]_{s}}(\mathrm{C})$. Put otherwise, a weak base 
$S$ is a base of $\operatorname{Inv}(\mathrm{C})$ with the largest possible number of partial polymorphisms. Hence, we obtain the following theorem.

Theorem 3.3 ([SS08]). Let $S$ be a weak base of a co-clone $\operatorname{Inv}(\mathrm{C})$, and let $S^{\prime}$ be an arbitrary base of $\operatorname{Inv}(\mathrm{C})$. Then

1. $S \subseteq\left\langle S^{\prime}\right\rangle_{\exists}$, and

2. $\operatorname{pPol}(S) \supseteq \operatorname{pPol}\left(S^{\prime}\right)$.

We now need a few additional definitions in order to explain Schnoor's and Schnoor's procedure for obtaining weak bases [SS08]. Given a natural number $\mu$ and a finite domain $D$ the $|D|^{\mu}$-ary relation $U_{D}^{\mu}$ is the relation which contains all natural numbers from 0 to $|D|^{\mu}-1$ as columns in the matrix representation. Thus, $U_{D}^{\mu}$ consists of the tuples $t_{1}, \ldots, t_{\mu}$ where for $1 \leq i \leq|D|^{\mu}$, the tuple $\left(t_{1}[i], \ldots, t_{\mu}[i]\right)$ is a representation of the natural number $i-1$. Since we in this chapter are only concerned with Boolean relations, we omit the domain $D$ in the subscript and simply write $U^{\mu}$ instead of $U_{\mathrm{B}}^{\mu}$.

Example 3.4. Consider the three relations

$$
U^{1}=\left(\begin{array}{ll}
0 & 1
\end{array}\right), U^{2}=\left(\begin{array}{llll}
0 & 0 & 1 & 1 \\
0 & 1 & 0 & 1
\end{array}\right), U^{3}=\left(\begin{array}{llllllll}
0 & 0 & 0 & 0 & 1 & 1 & 1 & 1 \\
0 & 0 & 1 & 1 & 0 & 0 & 1 & 1 \\
0 & 1 & 0 & 1 & 0 & 1 & 0 & 1
\end{array}\right)
$$

For any set of functions $F$ and relation $R$ we define $F(R)$ to be the relation

$$
\bigcap_{R^{\prime} \in \operatorname{Inv}(F), R \subseteq R^{\prime}} R^{\prime},
$$

i.e., the smallest extension of $R$ preserved by $F$. It is not difficult to see that if $[F]=\mathrm{C}$ then $F(R)=\mathrm{C}(R)[\mathrm{SS} 08]$. We say that a co-clone $\operatorname{Inv}(\mathrm{C})$ has coresize $S$ if there exist relations $R, R^{\prime}$ such that $\operatorname{Pol}(\{R\})=\mathrm{C}, R=\mathrm{C}\left(R^{\prime}\right)$, and $s=\left|R^{\prime}\right|$. The relation $R^{\prime}$ is in this case said to be a C-core of $R \mid$ SS08|. Minimal core-sizes for all Boolean co-clones of finite order have been identified by Schnoor [Sch08]. We are now ready to state Schnoor's and Schnoor's [SS08] main result, which effectively gives a weak base for any co-clone with a finite core-size.

Theorem 3.5 ([SS08]). Let $\mathrm{C}$ be a clone and let s be a core-size of $\operatorname{Inv}(\mathrm{C})$. Then the relation $\mathrm{C}\left(U^{s}\right)$ is a weak base of $\operatorname{Inv}(\mathrm{C})$.

A disadvantage of this theorem is that relations of the form $C\left(U^{s}\right)$ have exponential arity with respect to the core-size. We therefore introduce another measurement of minimality which ensures that a given relation is indeed minimal, in the sense that it does not contain any superfluous columns and that there is no subset of the relation which is still a weak base. A $\kappa$ ary relation $R$ is said to be irredundant if there are no duplicate columns in the matrix representation, and it is said to have a fictitious argument if 
there exists an $1 \leq i \leq \kappa$ such that $\left(a_{1}, \ldots, a_{i}, \ldots, a_{\kappa}\right) \in R$ if and only if $\left(a_{1}, \ldots, \overline{a_{i}}, \ldots, a_{\kappa}\right) \in R$.

Definition 3.6. A relation $R$ is minimal if it is (1) irredundant, (2) contains no fictitious arguments and (3) if there is no $R^{\prime} \subset R$ such that $\langle\{R\}\rangle=\left\langle\left\{R^{\prime}\right\}\right\rangle$.

Similarly, we say that a constraint language $S$ is minimal if every $R \in S$ is minimal. Clearly, our notion of minimality is not the only possible choice, but it is a useful definition since minimal weak bases have the property that they can be implemented without the use of explicit equality constraints. If we let $\langle\cdot\rangle_{\nexists \neq}$ denote the closure of q.f.p.p. definitions without equality, we therefore get the following theorem.

Theorem 3.7 ([SS08]). Let $\mathrm{C}$ be a clone and let $S$ be a minimal weak base of $\operatorname{Inv}(\mathrm{C})$. Then, for any base $S^{\prime}$ of $\operatorname{Inv}(\mathrm{C})$, it holds that $S \subseteq\left\langle S^{\prime}\right\rangle_{\nexists \neq}$.

Hence, minimal weak bases give the largest possible expressibility results and are applicable for problems such as counting $\operatorname{CSP}(\cdot)$, where equality constraints in an instance may increase the number of solutions exponentially [SS08].

\subsection{Minimal Weak Bases of Boolean Co-Clones}

In this section we proceed by giving minimal weak bases for all Boolean co-clones. The proofs are divided into Section 3.3.1. where we study Boolean co-clones of finite order, and Section 3.3.2. where we study Boolean co-clones of infinite order. The results are presented in Table 3.1. Each entry in the table consists of a co-clone, its minimal core-size, and a minimal weak base. The reader is advised to consult Section 2.3.2 for definitions of the involved relations. Variables are named $x_{1}, \ldots, x_{n}$ or $x$ except when they occur as arguments to $\mathrm{F}$ or $\mathrm{T}$ in which case they are named $c_{0}$ and $c_{1}$, respectively, to indicate that they are constants.

\subsubsection{The Finite Cases}

For the co-clones $I_{2}, I M, I D, I D_{1}$, IL, IL $\mathrm{IL}_{0}, \mathrm{IL}_{1}, \mathrm{IL}_{2}, \mathrm{IL}_{3}, I \mathrm{IV}, \mathrm{IV}_{0}, I \mathrm{IE}, \mathrm{IE}_{1}, \mathrm{IN}$, $\mathrm{IN}_{2}, \mathrm{II}, \mathrm{II}_{0}, \mathrm{II}_{1}$ and $\mathrm{BR}$, the result follows immediately from Theorem 3.5 , the minimal core-sizes for each co-clone of finite order [Sch08], and a suitable rearrangement of arguments. To make the thesis more self-contained, we give an example of how a minimal core-size of a co-clone can be determined and how it is used to compute a weak base.

Lemma 3.8. The bases for $\mathrm{IR}_{2}, \mathrm{IM}, \mathrm{ID}, \mathrm{ID}_{1}, \mathrm{IL}, \mathrm{IL}_{0}, \mathrm{IL}_{1}, \mathrm{IL}_{2}, \mathrm{IL}_{3}, \mathrm{IV}, \mathrm{IV} \mathrm{V}_{0}, \mathrm{IE}, \mathrm{IE}_{1}$, $\mathrm{IN}, \mathrm{IN}_{2}, \mathrm{II}, \mathrm{II}_{0}, \mathrm{II}_{1}$ and BR in Table 3.1 are minimal weak bases.

Proof. Let us consider $\mathrm{IN}_{2}$. Recall that $\mathrm{N}_{2}=[\{\neg x\}]$ and that $\left\langle\left\{R_{\mathrm{NAE}}\right\}\right\rangle=\mathrm{IN}_{2}$. Since $R_{\mathrm{NAE}}=\mathrm{N}_{2}\left(R_{1 / 3}\right)=\{n e g\}\left(R_{1 / 3}\right)$ and $\left|R_{1 / 3}\right|=3$ it follows that 3 is a core-size of $\mathrm{IN}_{2}$. Now, in order for 3 to also be a minimal core-size, we only 
have to verify that 1 and 2 are not core-sizes of $I N_{2}$. In the first case we see that $\mathrm{N}_{2}\left(U^{1}\right)=\langle\{(0,1),(1,0)\}\rangle=\mathrm{ID}$, and similarly, for the second case we also get that $N_{2}\left(U^{2}\right)=\langle\{(0,0,1,1),(0,1,0,1),(1,1,0,0),(1,0,1,0)\}\rangle=$ ID. We therefore get the weak base

$$
\mathrm{N}_{2}\left(U^{3}\right)=\left(\begin{array}{llllllll}
0 & 0 & 0 & 0 & 1 & 1 & 1 & 1 \\
0 & 0 & 1 & 1 & 0 & 0 & 1 & 1 \\
0 & 1 & 0 & 1 & 0 & 1 & 0 & 1 \\
1 & 1 & 1 & 1 & 0 & 0 & 0 & 0 \\
1 & 1 & 0 & 0 & 1 & 1 & 0 & 0 \\
1 & 0 & 1 & 0 & 1 & 0 & 1 & 0
\end{array}\right)
$$

by first calculating $U^{3}$ and then closing the resulting relation under unary complement. Let $R_{\mathrm{IN}_{2}}\left(x_{1}, x_{2}, x_{3}, x_{4}, x_{5}, x_{6}, x_{7}, x_{8}\right) \equiv$ 4 -EVEN ${ }^{4 \neq}\left(x_{1}, x_{2}, x_{3}, x_{4}, x_{5}, x_{6}, x_{7}, x_{8}\right) \wedge x_{1} x_{4} \leftrightarrow x_{2} x_{3}$ be the relation for $I_{2}$ in Table 3.1. It is then easily verified that

$$
R_{\mathrm{IN}_{2}}\left(x_{1}, x_{2}, x_{3}, x_{4}, x_{5}, x_{6}, x_{7}, x_{8}\right) \equiv \mathrm{N}_{2}\left(U^{3}\right)\left(x_{1}, x_{2}, x_{3}, x_{4}, x_{8}, x_{7}, x_{6}, x_{5}\right) .
$$

To see that $R_{\mathrm{IN}_{2}}$ is minimal in the sense of Definition 3.6. first observe that it is irredundant and does not contain any fictitious arguments. Second, observe there cannot exist an $R \subset R_{\mathrm{IN}_{2}}$ such that $\langle\{R\}\rangle=\left\langle\left\{R_{\mathrm{IN}_{2}}\right\}\right\rangle=\mathrm{IN}_{2}$, since this would imply that the core-size of $I \mathrm{~N}_{2}$ is smaller than 3 .

In general, the problem of checking whether a $\kappa$-ary relation $R$ is a base of a Boolean co-clone can be checked in time $O\left(\kappa^{2}|R|\right)$ using the algorithm in Creignou et al. $|\mathrm{CKZ08}|$, and through exhaustive search, i.e. by repeatedly removing redundant columns and tuples, one can verify that the bases are also minimal. This has been done by a computer program which is available at an online repository [Lag14].

For the remaining Boolean co-clones of finite order the proof is divided into two parts. First, we prove that the weak base for every co-clone IC $\in$ $\left\{\mathrm{IM}_{0}, \mathrm{IM}_{1}, \mathrm{IM}_{2}, \mathrm{ID}_{2}, \mathrm{IV}_{1}, \mathrm{IV}_{2}, \mathrm{IE}_{0}, \mathrm{IE}_{2}\right\}$, can be obtained by collapsing columns from $C\left(U^{s}\right)$, where $s$ is the minimal core-size of IC. Second, we prove that for every $\kappa \geq 2$ there exists simple weak bases for the co-clones $I \mathrm{I}_{0}^{\mathcal{K}}, \mathrm{IS}_{02}^{\mathcal{K}}, \mathrm{IS}_{01}^{\mathcal{K}}$, $I S_{00}^{\mathcal{K}}$ and their duals $I S_{1}^{\kappa}, I S_{12}^{\mathcal{K}}, I S_{11}^{\mathcal{K}}, I S_{10}^{\mathcal{K}}$. To make the proofs more concise we introduce some admissible operations on relations which preserve the weak base property. Let $R$ be a $\kappa$-ary relation. Each following rule of the form $R \stackrel{*}{\hookrightarrow} R^{\prime}$ implies that $\left\langle R^{\prime}\right\rangle_{\exists} \subseteq\langle R\rangle_{\exists}$.

- $R \stackrel{(i=j)}{\longrightarrow} R^{\prime}, 1 \leq i<j \leq \kappa$,

(Identify argument $i$ with argument $j$ ),

- $R \stackrel{\pi\left(i_{1}, \ldots, i_{\kappa}\right)}{\longrightarrow} R^{\prime}$, where $\pi$ is the permutation $\pi(j)=i_{j}, 1 \leq j \leq \kappa, 1 \leq$ $i_{j} \leq \kappa$, (Swap arguments according to $\pi$ ), 
Table 3.1: Minimal weak bases for all Boolean co-clones.

\begin{tabular}{|c|c|c|}
\hline Co-clone & Core-size & Weak base \\
\hline IBF & 1 & $\left\{\mathrm{Eq}\left(x_{1}, x_{2}\right)\right\}$ \\
\hline $\mathrm{IR}_{0}$ & 1 & $\left\{\mathrm{~F}\left(c_{0}\right)\right\}$ \\
\hline $\mathrm{IR}_{1}$ & 1 & $\left\{\mathrm{~T}\left(c_{1}\right)\right\}$ \\
\hline $\mathrm{IR}_{2}$ & 1 & $\left\{\mathrm{~F}\left(c_{0}\right) \wedge \mathrm{T}\left(c_{1}\right)\right\}$ \\
\hline IM & 1 & $\left\{\left(x_{1} \rightarrow x_{2}\right)\right\}$ \\
\hline $\mathrm{IM}_{0}$ & 2 & $\left\{\left(x_{1} \rightarrow x_{2}\right) \wedge \mathrm{F}\left(c_{0}\right)\right\}$ \\
\hline $\mathrm{IM}_{1}$ & 2 & $\left\{\left(x_{1} \rightarrow x_{2}\right) \wedge \mathrm{T}\left(c_{1}\right)\right\}$ \\
\hline $\mathrm{IM}_{2}$ & 3 & $\left\{\left(x_{1} \rightarrow x_{2}\right) \wedge \mathrm{F}\left(c_{0}\right) \wedge \mathrm{T}\left(c_{1}\right)\right\}$ \\
\hline $\mathrm{IS}_{0, \kappa}^{\kappa}, 2$ & $\kappa$ & $\left\{\mathrm{OR}^{\kappa}\left(x_{1}, \ldots, x_{\kappa}\right) \wedge \mathrm{T}\left(c_{1}\right)\right\}$ \\
\hline $\mathrm{IS}_{0}-$ & $\infty$ & $\left\{\mathrm{OR}^{\kappa}\left(x_{1}, \ldots, x_{\kappa}\right) \wedge \mathrm{T}\left(c_{1}\right) \mid \kappa \geq 2\right\}$ \\
\hline $\mathrm{IS}_{02}^{\kappa}, \kappa \geq 2$ & $\kappa$ & $\left\{\mathrm{OR}^{\kappa}\left(x_{1}, \ldots, x_{\kappa}\right) \wedge \mathrm{F}\left(c_{0}\right) \wedge \mathrm{T}\left(c_{1}\right)\right\}$ \\
\hline $\mathrm{IS}_{02}-\mathrm{C}$ & $\infty$ & $\left\{\mathrm{OR}^{\kappa}\left(x_{1}, \ldots, x_{\kappa}\right) \wedge \mathrm{F}\left(c_{0}\right) \wedge \mathrm{T}\left(c_{1}\right) \mid \kappa \geq 2\right\}$ \\
\hline $\mathrm{IS}_{01}^{\kappa}, \kappa>2$ & $\kappa$ & $\left\{\mathrm{OR}^{\kappa}\left(x_{1}, \ldots, x_{\kappa}\right) \wedge\left(x \rightarrow x_{1} \cdots x_{\kappa}\right) \wedge \mathrm{T}\left(c_{1}\right)\right\}$ \\
\hline $\mathrm{IS}_{01}$ & $\infty$ & $\left\{\mathrm{OR}^{\kappa}\left(x_{1}, \ldots, x_{\kappa}\right) \wedge\left(x \rightarrow x_{1} \cdots x_{\kappa}\right) \wedge \mathrm{T}\left(c_{1}\right) \mid \kappa \geq 2\right\}$ \\
\hline $\mathrm{IS}_{00, \kappa}^{\kappa}, \kappa 2$ & $\max (3, \kappa)$ & $\left\{\mathrm{OR}^{\kappa}\left(x_{1}, \ldots, x_{\kappa}\right) \wedge\left(x \rightarrow x_{1} \cdots x_{\kappa}\right) \wedge \mathrm{F}\left(c_{0}\right) \wedge \mathrm{T}\left(c_{1}\right)\right\}$ \\
\hline $\mathrm{IS}_{00}-$ & $\infty$ & $\left\{\mathrm{OR}^{\kappa}\left(x_{1}, \ldots, x_{\kappa}\right) \wedge\left(x \rightarrow x_{1} \cdots x_{\kappa}\right) \wedge \mathrm{F}\left(c_{0}\right) \wedge \mathrm{T}\left(c_{1}\right) \mid \kappa \geq 2\right\}$ \\
\hline $\mathrm{IS}_{1}^{\kappa}, \kappa \geq 2$ & $\kappa$ & $\left\{\operatorname{NAND}^{\kappa}\left(x_{1}, \ldots, x_{\kappa}\right) \wedge \mathrm{F}\left(c_{0}\right)\right\}$ \\
\hline $\mathrm{IS}_{1}-$ & $\infty$ & $\left\{\operatorname{NAND}^{\kappa}\left(x_{1}, \ldots, x_{\kappa}\right) \wedge \mathrm{F}\left(c_{0}\right) \mid \kappa \geq 2\right\}$ \\
\hline $\mathrm{IS}_{12}^{\kappa}, \kappa \geq 2$ & $\kappa$ & $\left\{\operatorname{NAND}^{\kappa}\left(x_{1}, \ldots, x_{\kappa}\right) \wedge \mathrm{F}\left(c_{0}\right) \wedge \mathrm{T}\left(c_{1}\right)\right\}$ \\
\hline $\mathrm{IS}_{12}$ & $\infty$ & $\left\{\operatorname{NAND}^{\kappa}\left(x_{1}, \ldots, x_{\kappa}\right) \wedge \mathrm{F}\left(c_{0}\right) \wedge \mathrm{T}\left(c_{1}\right) \mid \kappa \geq 2\right\}$ \\
\hline $\mathrm{IS}_{11, \kappa}^{\kappa}, \kappa \geq 2$ & $\kappa$ & $\left\{\operatorname{NAND}^{\kappa}\left(x_{1}, \ldots, x_{\kappa}\right) \wedge\left(x_{1} \rightarrow x\right) \wedge \ldots \wedge\left(x_{\kappa} \rightarrow x\right) \wedge \mathrm{F}\left(c_{0}\right)\right\}$ \\
\hline $\mathrm{IS}_{11}-\mathrm{-}$ & $\infty$ & $\left\{\operatorname{NAND}^{\kappa}\left(x_{1}, \ldots, x_{\kappa}\right) \wedge\left(x_{1} \rightarrow x\right) \wedge \ldots \wedge\left(x_{\kappa} \rightarrow x\right) \wedge \mathrm{F}\left(c_{0}\right) \mid \kappa>2\right\}$ \\
\hline $\mathrm{IS}_{10}^{\kappa}, \kappa \geq 2$ & $\max (3, \kappa)$ & $\left\{\operatorname{NAND}^{\kappa}\left(x_{1}, \ldots, x_{\kappa}\right) \wedge\left(x_{1} \rightarrow x\right) \wedge \ldots \wedge\left(x_{\kappa} \rightarrow x\right) \wedge \mathrm{F}\left(c_{0}\right) \wedge \mathrm{T}\left(c_{1}\right)\right\}$ \\
\hline $\mathrm{IS}_{10}$ & $\infty$ & $\left\{\operatorname{NAND}^{\kappa}\left(x_{1}, \ldots, x_{\kappa}\right) \wedge\left(x_{1} \rightarrow x\right) \wedge \ldots \wedge\left(x_{\kappa} \rightarrow x\right) \wedge \mathrm{F}\left(c_{0}\right) \wedge \mathrm{T}\left(c_{1}\right) \mid \kappa \geq 2\right\}$ \\
\hline ID & 1 & $\left\{\operatorname{Neq}\left(x_{1}, x_{2}\right)\right\}$ \\
\hline$I_{1}$ & 2 & $\left\{\operatorname{Neq}\left(x_{1}, x_{2}\right) \wedge \mathrm{F}\left(c_{0}\right) \wedge \mathrm{T}\left(c_{1}\right)\right\}$ \\
\hline$I_{2}$ & 3 & $\left\{\mathrm{OR}^{2}\left(x_{1}, x_{2}\right) \wedge \operatorname{Neq}\left(x_{1}, x_{3}\right) \wedge \operatorname{Neq}\left(x_{2}, x_{3}\right) \wedge \mathrm{F}\left(c_{0}\right) \wedge \mathrm{T}\left(c_{1}\right)\right\}$ \\
\hline IL & 2 & $\left\{4-\operatorname{EVEN}\left(x_{1}, x_{2}, x_{3}, x_{4}\right)\right\}$ \\
\hline $\mathrm{IL}_{0}$ & 2 & $\left\{3-\operatorname{EVEN}\left(x_{1}, x_{2}, x_{3}\right) \wedge \mathrm{F}\left(c_{0}\right)\right\}$ \\
\hline $\mathrm{IL}_{1}$ & 2 & $\left\{3-\mathrm{ODD}\left(x_{1}, x_{2}, x_{3}\right) \wedge \mathrm{T}\left(c_{1}\right)\right\}$ \\
\hline $\mathrm{IL}_{2}$ & 3 & $\left\{3-\operatorname{EVEN}^{3 \neq}\left(x_{1}, \ldots, x_{6}\right) \wedge \mathrm{F}\left(c_{0}\right) \wedge \mathrm{T}\left(c_{1}\right)\right\}$ \\
\hline $\mathrm{IL}_{3}$ & 3 & $\left\{4-\operatorname{EVEN}^{4 \neq}\left(x_{1}, \ldots, x_{8}\right)\right\}$ \\
\hline IV & 2 & $\left\{\left(\overline{x_{1}} \leftrightarrow \overline{x_{2}} \overline{x_{3}}\right) \wedge\left(\overline{x_{2}} \vee \overline{x_{3}} \rightarrow \overline{x_{4}}\right)\right\}$ \\
\hline $\mathrm{IV}_{0}$ & 2 & $\left\{\left(\overline{x_{1}} \leftrightarrow \overline{x_{2}} \overline{x_{3}}\right) \wedge \mathrm{F}\left(c_{0}\right)\right\}$ \\
\hline $\mathrm{IV}_{1}$ & 3 & $\left\{\left(\overline{x_{1}} \leftrightarrow \overline{x_{2}} \overline{x_{3}}\right) \wedge\left(\overline{x_{2}} \vee \overline{x_{3}} \rightarrow \overline{x_{4}}\right) \wedge \mathrm{T}\left(c_{1}\right)\right\}$ \\
\hline $\mathrm{IV}_{2}$ & 3 & $\left\{\left(\overline{x_{1}} \leftrightarrow \overline{x_{2}} \overline{x_{3}}\right) \wedge \mathrm{F}\left(c_{0}\right) \wedge \mathrm{T}\left(c_{1}\right)\right\}$ \\
\hline IE & 2 & $\left\{\left(x_{1} \leftrightarrow x_{2} x_{3}\right) \wedge\left(x_{2} \vee x_{3} \rightarrow x_{4}\right)\right\}$ \\
\hline $\mathrm{IE}_{0}$ & 3 & $\left\{\left(x_{1} \leftrightarrow x_{2} x_{3}\right) \wedge\left(x_{2} \vee x_{3} \rightarrow x_{4}\right) \wedge \mathrm{F}\left(c_{0}\right)\right\}$ \\
\hline $\mathrm{IE}_{1}$ & 2 & $\left\{\left(x_{1} \leftrightarrow x_{2} x_{3}\right) \wedge \mathrm{T}\left(c_{1}\right)\right\}$ \\
\hline $\mathrm{IE}_{2}$ & 3 & $\left\{\left(x_{1} \leftrightarrow x_{2} x_{3}\right) \wedge \mathrm{F}\left(c_{0}\right) \wedge \mathrm{T}\left(c_{1}\right)\right\}$ \\
\hline IN & 2 & $\left\{4-\operatorname{EVEN}\left(x_{1}, x_{2}, x_{3}, x_{4}\right) \wedge x_{1} x_{4} \leftrightarrow x_{2} x_{3}\right\}$ \\
\hline $\mathrm{IN}_{2}$ & 3 & $\left\{4-\operatorname{EVEN}^{4 \neq}\left(x_{1}, \ldots, x_{8}\right) \wedge x_{1} x_{4} \leftrightarrow x_{2} x_{3}\right\}$ \\
\hline 11 & 2 & $\left\{\left(x_{1} \leftrightarrow x_{2} x_{3}\right) \wedge\left(\overline{x_{4}} \leftrightarrow \overline{x_{2}} \overline{x_{3}}\right)\right\}$ \\
\hline $\mathrm{II}_{0}$ & 2 & $\left\{\left(\overline{x_{1}} \vee \overline{x_{2}}\right) \wedge\left(\overline{x_{1}} \overline{x_{2}} \leftrightarrow \overline{x_{3}}\right) \wedge \mathrm{F}\left(c_{0}\right)\right\}$ \\
\hline $\mathrm{II}_{1}$ & 2 & $\left\{\left(x_{1} \vee x_{2}\right) \wedge\left(x_{1} x_{2} \leftrightarrow x_{3}\right) \wedge \mathrm{T}\left(c_{1}\right)\right\}$ \\
\hline $\mathrm{BR}$ & 3 & $\left\{R_{1 / 3}^{\neq \neq \neq}\left(x_{1}, \ldots, x_{6}\right) \wedge \mathrm{F}\left(c_{0}\right) \wedge \mathrm{T}\left(c_{1}\right)\right\}$ \\
\hline
\end{tabular}




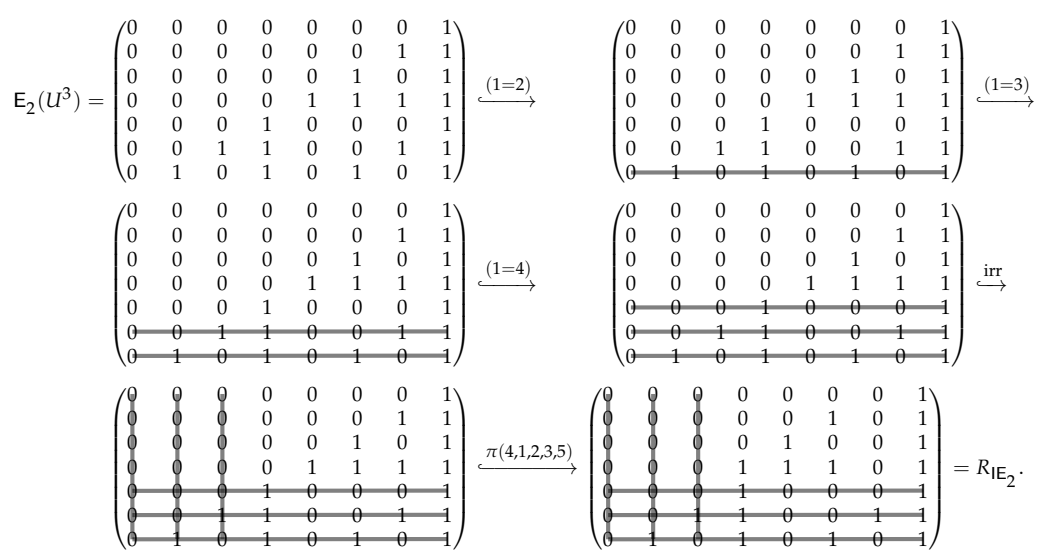

Figure 3.1: Reduction sequence for $R_{\mathrm{IE}_{2}}$. A black line indicates that the column/row has been removed from the relation.

- $R \stackrel{\text { irr }}{\longrightarrow} R^{\prime}$,

( $R^{\prime}$ is irredundant).

Lemma 3.9. Let IC be a co-clone, $R$ a $\kappa$-ary weak base of IC, and let $R^{\prime}$ be a relation such that $R \stackrel{*}{\hookrightarrow} R^{\prime}$ for some rule $\stackrel{*}{\hookrightarrow}$. If $R^{\prime}$ is a base of IC then it is also a weak base of IC.

Proof. We prove that $\langle\{R\}\rangle_{\nexists \exists}=\left\langle\left\{R^{\prime}\right\}\right\rangle_{\nexists}$ which implies that $R^{\prime}$ is a weak base of IC. The first inclusion $\langle R\rangle_{\exists} \subseteq\left\langle R^{\prime}\right\rangle_{\exists}$ is obvious since $R$ is a weak base by assumption. To prove that $\left\langle\left\{R^{\prime}\right\}\right\rangle_{\nexists} \subseteq\langle\{R\}\rangle_{\exists}$ we show that $R^{\prime} \in\langle\{R\}\rangle_{\nexists}$ by giving a q.f.p.p. implementation of $R^{\prime}$ with $R$. There are three cases to consider. Either

$$
R \stackrel{(i=j)}{\longrightarrow} R^{\prime}, 1 \leq i<j \leq \kappa,
$$

in which case $R^{\prime}$ is the $\kappa$-ary relation defined as

$$
R^{\prime}\left(x_{1}, \ldots, x_{i}, \ldots, x_{j}, \ldots, x_{\kappa}\right) \equiv \operatorname{Eq}\left(x_{i}, x_{j}\right) \wedge R\left(x_{1}, \ldots, x_{i}, \ldots, x_{j}, \ldots, x_{\kappa}\right),
$$

or

$$
R \stackrel{\pi\left(i_{1}, \ldots, i_{\kappa}\right)}{\longrightarrow} R^{\prime} \text { for some permutation } \pi,
$$

in which case

$$
R^{\prime}\left(x_{1}, \ldots, x_{\kappa}\right) \equiv R\left(x_{\pi(1)}, \ldots, x_{\pi(\kappa)}\right) .
$$

The case when $R^{\prime}$ is irredundant follows easily since it can be obtained from $R$ by identifying all duplicate arguments. 
Lemma 3.10. The bases for $\mathrm{IM}_{0}, \mathrm{IM}_{1}, \mathrm{IM}_{2}, \mathrm{ID}_{2}, \mathrm{IV}_{1}, \mathrm{IV}_{2}, \mathrm{IE}_{0}$ and $\mathrm{IE}_{2}$ in Table 3.1 are minimal weak bases.

Proof. We consider each case in turn. For every co-clone IC we write $R_{\mathrm{IC}}$ for the weak base from Table 3.1. We begin with the derivation for $R_{\mathrm{IE}_{2}}$. From Table 3.1 we see that the core-size of $\mathrm{IE}_{2}$ is 3 , which means that we need to begin with the relation $U^{3}=\{(0,0,0,0,1,1,1,1)$, $(0,0,1,1,0,0,1,1),(0,1,0,1,0,1,0,1)\}$. The base of $E_{2}$ is according to Table 2.1 in Chapter 2 the binary function $f\left(x_{1}, x_{2}\right)=x_{1} \wedge x_{2}$. When calculating $\mathrm{E}_{2}\left(U^{3}\right)$ we thus need to close $U^{3}$ under $f$, which we do by repeatedly applying $f$ to the tuples of $U^{3}$ until no new tuples can be obtained, which means that the resulting relation is closed under $f$. In the case of $\mathrm{E}_{2}\left(U^{3}\right)$ we obtain four new tuples and get the relation $\mathrm{E}_{2}\left(U^{3}\right)=U^{3} U$ $\{(0,0,0,0,1,1,1,1),(0,0,0,1,0,0,0,1),(0,0,1,1,0,0,1,1),(0,1,0,1,0,1,0,1)\}$. By identifying arguments, removing redundant arguments and permutating arguments it is then possible to derive $R_{\mathrm{IE}_{2}}$. The sequence is visualised in Figure 3.3.1.

The reductions for the remaining co-clones are included below, where $R$, $R^{\prime}, R^{\prime \prime}, R^{\prime \prime \prime}$, and $R^{\prime \prime \prime \prime}$ denotes intermediate relations.

$$
\begin{aligned}
& \mathrm{R}_{0}\left(U^{1}\right) \stackrel{(1=2)}{\longrightarrow} R \stackrel{\text { irr }}{\longrightarrow} R_{\mathrm{IR}_{0}} \text {. } \\
& \mathrm{R}_{1}\left(U^{1}\right) \stackrel{(1=2)}{\longrightarrow} R \stackrel{\text { irr }}{\hookrightarrow} R_{\mathrm{IR}_{1}} \text {. } \\
& \mathrm{M}_{0}\left(U^{2}\right) \stackrel{(1=2)}{\longrightarrow} R \stackrel{\mathrm{irr}}{\longleftrightarrow} R^{\prime} \stackrel{\pi(3,1,2)}{\longrightarrow} R_{\mathrm{IM}_{0}} \text {. } \\
& \mathrm{M}_{1}\left(U^{2}\right) \stackrel{(1=2)}{\longrightarrow} R \stackrel{\text { irr }}{\longleftrightarrow} R_{\mathrm{IM}_{1}} \text {. } \\
& \mathrm{M}_{2}\left(U^{3}\right) \stackrel{(1=2)}{\longrightarrow} R \stackrel{(2=3)}{\longrightarrow} R^{\prime} \stackrel{(3=4)}{\longrightarrow} R^{\prime \prime} \stackrel{(5=6)}{\longrightarrow} R^{\prime \prime \prime} \stackrel{\text { irr }}{\longrightarrow} R^{\prime \prime \prime \prime \prime} \stackrel{\pi(3,1,2,4)}{\longrightarrow} R_{\mathrm{IM}_{2}} \text {. } \\
& \mathrm{D}_{2}\left(U^{3}\right) \stackrel{(1=2)}{\longrightarrow} R \stackrel{\mathrm{irr}}{\longleftrightarrow} R^{\prime} \stackrel{\pi(5,4,1,3,2,6)}{\longrightarrow} R_{\mathrm{ID}_{2}} \text {. } \\
& \mathrm{V}_{1}\left(U^{3}\right) \stackrel{(4=8)}{\longrightarrow} R \stackrel{(2=6)}{\longrightarrow} R^{\prime} \stackrel{(6=8)}{\longrightarrow} R^{\prime \prime} \stackrel{\operatorname{irr}}{\longrightarrow} R^{\prime \prime \prime} \stackrel{\pi(4,2,3,1,5)}{\longrightarrow} R_{\mathrm{IV}_{1}} \text {. } \\
& \mathrm{V}_{2}\left(U^{3}\right) \stackrel{(4=8)}{\longrightarrow} R \stackrel{(2=6)}{\longrightarrow} R^{\prime} \stackrel{(2=8)}{\longrightarrow} R^{\prime \prime} \stackrel{\text { irr }}{\longrightarrow} R^{\prime \prime \prime} \stackrel{\pi(4,2,3,1,5)}{\longrightarrow} R_{\mathrm{IV}_{2}} \text {. } \\
& \mathrm{E}_{0}\left(U^{3}\right) \stackrel{(1=2)}{\longrightarrow} R \stackrel{(2=3)}{\longrightarrow} R^{\prime} \stackrel{(3=4)}{\longrightarrow} R^{\prime \prime} \stackrel{\text { irr }}{\longrightarrow} R^{\prime \prime \prime} \stackrel{\pi(5,1,2,3,4)}{\longrightarrow} R_{\mathrm{IE}_{0}} .
\end{aligned}
$$

It is not hard to see that every relation $R_{\mathrm{IC}}$ is a base of IC. As in the previous cases the minimality of each weak base can be verified through exhaustive search. As an example, again consider

$$
R_{\mathrm{IE}_{2}}=\left(\begin{array}{ccccc}
0 & 0 & 0 & 0 & 1 \\
0 & 0 & 1 & 0 & 1 \\
0 & 1 & 0 & 0 & 1 \\
1 & 1 & 1 & 0 & 1
\end{array}\right)
$$

First note that $R_{\mathrm{IE}_{2}}$ has no redundant or fictitious arguments. As for subset minimality removing three rows results in a relation in $I R_{2}$ while removing two rows from $R_{\mathrm{IE}_{2}}$ results in a relation in $\mathrm{ID}_{1}$. Removing the first row results in a relation which generates $B R$ and is therefore no longer included in $I E_{2}$, 
removing the second or third row gives a relation in $\mathrm{IM}_{2}$, and removing the fourth row gives a relation in $I S_{10}^{2}$. These properties can efficiently be tested using the algorithm in Creignou et al. [CKZ08]. Hence, there is no relation $R^{\prime} \subset R_{\mathrm{IE}_{2}}$ such that $\left\langle\left\{R^{\prime}\right\}\right\rangle=\mathrm{IE}_{2}$ from which it follows that $R_{\mathrm{IE}_{2}}$ is a minimal weak base.

We now turn our attention towards the eight infinite chains of co-clones in Figure 2.2. In the sequel we sometimes represent relations by formulas in conjunctive normal form. If $\mathbf{x}=x_{1}, \ldots, x_{\kappa}$ we use $\varphi(\mathbf{x})$ to denote a formula with $\kappa$ free variables, and the formula $\varphi$ is then interpreted as defining a $\kappa$ ary relation where the tuples coincide with the satisfying assignments of $\varphi$. If $\varphi=C_{1} \wedge \ldots \wedge C_{\mu}$ is a formula with $\mu$ clauses we say that $C_{i}$ is a prime implicate of $\varphi$ if $\varphi$ does not entail any proper subclause of $C_{i}$. A formula $\varphi$ is said to be prime if all of its clauses are prime implicates. Obviously, any finite Boolean relation is representable by a prime formula. If $R$ is a $\kappa$-ary Boolean relation we can therefore prove that $R \in\langle S\rangle_{\exists}$ by showing that $R\left(x_{1}, \ldots, x_{\mathcal{K}}\right)$ can be expressed as a conjunction $\varphi_{1}\left(\mathbf{y}_{\mathbf{1}}\right) \wedge \ldots \wedge \varphi_{\mu}\left(\mathbf{y}_{\mu}\right)$, where each $\mathbf{y}_{\mathbf{i}}$ is a tuple of variables over $x_{1}, \ldots, x_{\kappa}$, and each $\varphi_{i}$ is a prime formula representation of a relation in $S$. This is advantageous since relations in $\mathrm{IS}_{0}^{\mathcal{K}}, \mathrm{IS}_{02}^{\mathcal{K}}, \mathrm{IS}_{01}^{\mathcal{K}}, \mathrm{IS}_{00}^{\mathcal{K}}, \mathrm{IS}_{1}^{\mathcal{K}}$, $\mathrm{IS}_{12}^{\kappa}, \mathrm{IS}_{11}^{\kappa}$ and $\mathrm{IS}_{10}^{\kappa}$ are representable by prime implicative hitting set-bounded (IHSB) formulas [CKZ08]. We let $\mathrm{IHSB}_{+}^{\kappa}$ be the set of formulas of the form $\left(x_{1} \vee \ldots \vee x_{\lambda}\right), 1 \leq \lambda \leq \kappa,\left(\neg x_{1}\right),\left(\neg x_{1} \vee x_{2}\right)$, and dually for IHSB IH $_{-}$.

Lemma 3.11. For each $\kappa \geq 2$ the relations for $\mathrm{IS}_{0}^{\kappa}, \mathrm{IS}_{02}^{\kappa}, \mathrm{IS}_{01}^{\kappa}$, and $\mathrm{IS}_{00}^{\kappa}$ in Table 3.1 are minimal weak bases.

Proof. To avoid repetition, we only present the full proof for $\mathrm{IS}_{00}^{\kappa}$. For $\mathrm{IS}_{0}^{\kappa}$, $\mathrm{IS}_{02}^{\kappa}$, and $\mathrm{IS}_{01}^{\kappa}$, the proofs are entirely analogous, but with a slightly different case analysis.

Let $R_{\mathrm{IS}}^{\kappa}$ denote the relation for $\mathrm{IS}_{00}^{\kappa}$ from Table 3.1, i.e., $R_{\mathrm{IS}_{00}^{\kappa}}\left(x_{1}, \ldots, x_{\kappa}, c_{0}, c_{1}\right) \equiv \mathrm{OR}^{\kappa}\left(x_{1}, \ldots, x_{\kappa}\right) \wedge\left(x \rightarrow x_{1} \cdots x_{\kappa}\right) \wedge \mathrm{F}\left(c_{0}\right) \wedge \mathrm{T}\left(c_{1}\right)$. Let $S$ be a constraint language such that $\langle S\rangle=\mathrm{IS}_{00}^{\kappa}$. We make two observations before the proof. First, since $I S_{00}^{\kappa}$ is of finite order we may assume that $S$ is finite, since otherwise there exists a finite $S^{\prime} \subseteq S$ such that $\left\langle S^{\prime}\right\rangle=\mid \mathrm{I}_{00}^{\kappa}$. Second, since $S$ is finite the relation $R$ defined to be the Cartesian product of all relations in $S$ satisfies $\langle\{R\}\rangle_{\exists} \subseteq\langle S\rangle_{\exists}$. Hence, if $R_{\mathrm{IS}_{00}^{\kappa}} \in\langle\{R\}\rangle_{\exists}$ then $R_{\mathrm{IS}_{00}^{\kappa}} \in\langle S\rangle_{\nexists \exists}$. In the rest of this proof, let $\lambda>\kappa$ denote the arity of $R$.

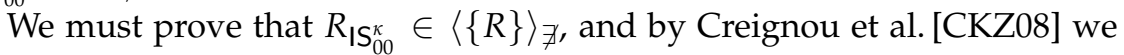
know that $R$ can be expressed as an $\operatorname{IHSB}_{+}^{\kappa}$ formula $\varphi\left(y_{1}, \ldots, y_{\lambda}\right)$. The strategy is therefore to prove that $R_{\mathrm{IS}_{00}^{\kappa}}$ can be expressed as a conjunction of $\varphi$ formulas without introducing any existentially quantified variables.

We first implement $\mathrm{F}\left(c_{0}\right)$ with $\varphi\left(y_{1}, \ldots, y_{\lambda}\right)$ by identifying every variable $y_{j}$ occurring in a negative clause $\left(\neg y_{j}\right)$ to $c_{0}$. There must exist at least one negative unary clause in $\varphi$ since otherwise $\langle\{R\}\rangle=\mathrm{IS}_{01}^{\mathcal{K}}$. Then, for any implicative clause $\left(\neg y_{j} \vee c_{0}\right)$ which also entails $\left(\neg c_{0} \vee y_{j}\right)$ we identify $y_{j}$ with $c_{0}$. For any remaining clause we identify all unbound variables with $c_{1}$. Since 
there must exist at least one positive clause this correctly implements $\mathrm{T}\left(c_{1}\right)$. Let $\varphi_{\mathrm{F}, \mathrm{T}}\left(c_{0}, c_{1}\right)$ denote the resulting formula.

To implement $\mathrm{OR}^{\kappa}\left(x_{1}, \ldots, x_{\kappa}\right)$, we first note that $\varphi$ must contain a prime implicate of the form $\left(y_{j_{1}} \vee \ldots \vee y_{j_{\kappa}}\right)$. We therefore identify $y_{j_{1}}, \ldots, y_{j_{\kappa}}$ with $x_{1}, \ldots, x_{\kappa}$. Let the resulting formula be $\varphi^{\prime}$. Note that $\varphi^{\prime}$ might still contain variables distinct from $x_{1}, \ldots, x_{\kappa}$. In the subsequent proof we use $x_{i}$ and $x_{i^{\prime}}$ to denote variables in $x_{1}, \ldots, x_{\kappa}$ and $y_{j}, y_{j^{\prime}}$ to denote variables in $\varphi^{\prime}$ distinct from $x_{1}, \ldots, x_{\kappa}$. Hence, we need to replace each $y_{i}$ still occurring in $\varphi^{\prime}$ with some $x_{i}, c_{0}, c_{1}$, or $x$. For every implicative clause $C$ there are then three cases to consider.

1. $\mathrm{C}=\left(\neg x_{i} \vee x_{i^{\prime}}\right)$,

2. $C=\left(\neg x_{i} \vee y_{j}\right)$,

3. $\mathrm{C}=\left(\neg y_{j} \vee x_{i}\right)$.

The first case is impossible since $\left(x_{1} \vee \ldots \vee x_{\kappa}\right)$ was assumed to be a prime implicate. This also implies that the clauses $\left(\neg x_{i} \vee y_{j}\right)$ and $\left(\neg y_{j} \vee x_{i^{\prime}}\right)$ cannot occur simultaneously in the formula. In the second case we identify $y_{j}$ with $c_{1}$, and to handle the third case we identify $y_{j}$ with $c_{0}$. If both the second and third case occur simultaneously we identify $y_{j}$ with $x_{i}$. For any remaining clause we identify each unbound $y_{j}$ with either $c_{0}$ or $c_{1}$. Thus, the resulting formula $\varphi_{\mathrm{OR}}\left(x_{1}, \ldots, x_{\kappa}, c_{0}, c_{1}\right)$ implements $\mathrm{OR}\left(x_{1}, \ldots, x_{\kappa}\right)$.

In order to implement $\left(x \rightarrow x_{1} \cdots x_{\kappa}\right) \equiv\left(\neg x \vee\left(x_{1} \wedge \ldots \wedge x_{\kappa}\right)\right)$ we need to ensure that $\neg x \vee x_{i}$ for all $1 \leq i \leq \kappa$. Since $\langle\{R\}\rangle=\mathrm{IS}_{00}^{\mathcal{K}}$ the prime formula representation $\varphi\left(y_{1}, \ldots, y_{\lambda}\right)$ of $R$ must contain a prime implicate of the form $\left(\neg y_{j_{1}} \vee y_{j_{2}}\right)$, where $\varphi$ does not entail $\left(\neg y_{j_{2}} \vee y_{j_{1}}\right)$. To implement $\left(\neg x \vee x_{i}\right)$ we therefore identify $y_{j_{1}}$ with $x$ and $y_{j_{2}}$ with $x_{i}$. In the subsequent formula there are then four implicative cases to consider:

1. $C=\left(\neg x \vee y_{j}\right)$,

2. $C=\left(\neg y_{j} \vee x\right)$,

3. $C=\left(\neg x_{i} \vee y_{j}\right)$,

4. $C=\left(\neg y_{j} \vee x_{i}\right)$,

where $y_{j}$ denotes a variable distinct from $x_{i}$ and $x$. In the first case we identify $y_{j}$ with $x_{i}$, if the second case occurs we identify $y_{j}$ with $c_{0}$, and if both the first and second case occur simultaneously we identify $y_{j}$ with $x$. In the third case we identify $y_{j}$ with $c_{1}$, in the fourth case we identify $y_{j}$ with $x$, and if both cases occur we identify $y_{j}$ with $x_{i}$. For any remaining positive clause we identify each unbound variable to $c_{1}$, and for any remaining negative unary clause $\left(\neg y_{j}\right)$ we identify $y_{j}$ with $c_{0}$. In case there still exists an implicative clause $\left(\neg y_{j} \vee y_{j^{\prime}}\right)$ we identify $y_{j}$ with $c_{0}$ and $y_{j^{\prime}}$ with $c_{1}$. Let the resulting formula be $\varphi_{\rightarrow}\left(x, x_{i}, c_{0}, c_{1}\right)$. If we repeat the procedure for all $1 \leq i \leq \kappa$ we see that $\varphi_{\rightarrow}\left(x, x_{1}, c_{0}, c_{1}\right) \wedge \ldots \wedge \varphi_{\rightarrow}\left(x, x_{\kappa}, c_{0}, c_{1}\right)$ 
implements $\left(\neg x \vee\left(x_{1} \wedge \ldots \wedge x_{\kappa}\right)\right)$. Put together, the formula $\varphi_{\mathrm{F}, \mathrm{T}}\left(c_{0}, c_{1}\right) \wedge$ $\varphi_{\mathrm{OR}}\left(x_{1}, \ldots, x_{\kappa}, c_{0}, c_{1}\right) \wedge \varphi_{\rightarrow}\left(x, x_{1}, c_{0}, c_{1}\right) \wedge \ldots \wedge \varphi_{\rightarrow}\left(x, x_{\kappa}, c_{0}, c_{1}\right)$ correctly implements $R_{\mathrm{IS}_{00}^{\kappa}}$, and furthermore only contains variables from $x_{1}, \ldots, x_{\kappa}, x$, $c_{0}, c_{1}$. One can also prove that $R_{\mathrm{IS}}^{\kappa}$ is a base of $\mathrm{IS}_{00}^{\kappa}$ by giving an explicit p.p. definition of the base given by Böhler et al.[BSRV05]. As for minimality, first note that $R_{\mathrm{IS}_{00}^{\kappa}}$ does not contain any redundant or fictitious arguments and a case study similar to that of the preceding proof shows that removing any number of tuples from $R_{\mathrm{IS}_{00}^{\kappa}}$ results in a relation which either (1) does not contain a $\kappa$-ary prime implicate $\left(x_{1} \vee \ldots \vee x_{\kappa}\right)$ or (2) does not contain an implicative prime clause or (3) can no longer be expressed as an $\mathrm{IHSB}_{+}^{\kappa}$ formula.

For the co-clones $\mathrm{IS}_{1}^{\kappa}, \mathrm{IS}_{12}^{\kappa}, \mathrm{IS}_{11}^{\kappa}$, and $\mathrm{IS}_{10}^{\kappa}$ we use a similar argument, but instead use the fact that the relations in these cases can be represented as restricted $\mathrm{IHSB}_{-}^{\kappa}$ formulas.

Lemma 3.12. For each $\kappa \geq 2$ the relations for $\mathrm{IS}_{1}^{\kappa}, \mathrm{IS}_{12}^{\kappa}, \mathrm{IS}_{11}^{\kappa}$, and $\mathrm{IS}_{10}^{\kappa}$, in Table 3.1 are minimal weak bases.

Proof. We give a proof for $\mathrm{IS}_{11}^{\mathcal{K}}$ since this is the case which differs the most from Lemma 3.11. Let $R_{\mathrm{IS}_{11}^{\kappa}}\left(x_{1}, \ldots, x_{\kappa}, x, c_{0}\right) \equiv \operatorname{NAND}^{\kappa}\left(x_{1}, \ldots, x_{\kappa}\right) \wedge\left(x_{1} \rightarrow\right.$ $x) \wedge \ldots \wedge\left(x_{\kappa} \rightarrow x\right) \wedge \mathrm{F}\left(c_{0}\right)$ denote the relation for $\mathrm{IS}_{11}^{\kappa}$ from Table 3.1. Let $S$ be a base of $I S_{11}^{\kappa}$. Similar to the case of Lemma 3.11. we may without loss of generality assume that $S=\{R\}$ for a $\lambda$-ary relation $R$, where $\lambda>\kappa$. We exploit the fact that $R$ can be expressed as a prime IHSB $_{-}^{\kappa}$ formula $\varphi\left(y_{1}, \ldots, y_{\lambda}\right)$ where each clause is of the form (1) $\left(\neg y_{j_{1}} \vee \ldots \vee \neg y_{j_{k}}\right)$ or (2) $\left(\neg y_{j_{1}} \vee y_{j_{2}}\right)$ |CKZ08|. We must therefore prove that $R_{\mathrm{IS}} \mathrm{S}_{11}\left(x_{1}, \ldots, x_{\kappa}, x, c_{0}\right) \equiv$ $\varphi_{1} \wedge \ldots \wedge \varphi_{\mu}$ where each $\varphi_{i}$ is an $\mathrm{IHSB}_{-}^{\kappa}$ representation of $R$ over the variables $x_{1}, \ldots, x_{\kappa}, x, c_{0}$. In the following three cases, let $\varphi\left(y_{1}, \ldots, y_{\lambda}\right)$ be an $\mathrm{IHSB}_{-}^{\kappa}$ representation of $R$.

First, we show how to implement $\mathrm{F}\left(c_{0}\right)$. Since $\langle\{R\}\rangle=\mathrm{IS}_{11}^{\kappa}, \varphi$ must contain a unary clause of the form $\left(\neg y_{j}\right)$. Hence, identify every variable in $\varphi$ with $c_{0}$, and let $\varphi_{\mathrm{F}}\left(c_{0}\right)$ denote the resulting formula. It is easy to see that $\varphi_{\mathrm{F}}\left(c_{0}\right)$ is satisfiable if and only if $c_{0}$ is assigned the value 0 .

Second, we have to implement $\operatorname{NAND}^{\kappa}\left(x_{1}, \ldots, x_{\kappa}\right)$ using $\varphi\left(y_{1}, \ldots, y_{\lambda}\right)$. We begin by identifying every variable $y_{j}$ such that $\varphi$ entails $\neg y_{j}$ with $c_{0}$. Let $\varphi^{\prime}$ denote the resulting formula. Using a similar argument as in Lemma 3.11 we then see that $\varphi^{\prime}$ must contain a prime implicate of the form $\left(\neg y_{j_{1}} \vee \ldots \vee\right.$ $\neg y_{j_{\kappa}}$ ). Hence, we identify $x_{1}, \ldots, x_{\kappa}$ with $y_{j_{1}}, \ldots, y_{j_{\kappa}}$ and let $\varphi^{\prime \prime}$ denote the resulting formula. We must now ensure that any remaining variable $y_{j}$ in $\varphi^{\prime \prime}$ which is distinct from $x_{1}, \ldots, x_{\kappa}$ or $c_{0}$, is identified to some $x_{i}, c_{0}$ or $x$. For each implicative clause $C$ containing variables $x_{i}, x_{i}$ from $x_{1}, \ldots, x_{\kappa}$, there are now three cases to consider.

1. $C=\left(\neg x_{i} \vee x_{i^{\prime}}\right)$,

2. $C=\left(\neg x_{i} \vee y_{j}\right)$, 


\section{3. $C=\left(\neg y_{j} \vee x_{i}\right)$,}

where $y_{j}$ denotes a variable distinct from $x_{1}, \ldots, x_{k}$. The first case cannot occur since this implies that $\left(\neg x_{1} \vee \ldots \vee \neg x_{\kappa}\right)$ is not a prime implicate. If both the second and third case occur simultaneously we identify $y_{j}$ with $x_{i}$. If only the second case occurs we identify $y_{j}$ with $x$, and if only the third case occurs we identify $y_{j}$ with $c_{0}$. We then identify any remaining variable $y_{j}$ with $c_{0}$, and let $\varphi_{\mathrm{NAND}}\left(x_{1}, \ldots, x_{\kappa}, x, c_{0}\right)$ denote the resulting formula. Note that $\varphi_{\mathrm{NAND}}\left(x_{1}, \ldots, x_{\kappa}, x, c_{0}\right)$ might enforce constraints of the form $\left(x_{i} \rightarrow x\right)$ for $x_{i} \in\left\{x_{1}, \ldots, x_{\kappa}\right\}$.

Third, implementing $\left(x_{i} \rightarrow x\right)$ for every $x_{i} \in\left\{x_{1}, \ldots, x_{\kappa}\right\}$ is similar to the strategy in Lemma 3.11. since $\langle\{R\}\rangle=\mathrm{IS}_{11}^{\kappa}$ the prime formula representation $\varphi\left(y_{1}, \ldots, y_{\lambda}\right)$ of $R$ must contain a clause of the form $\left(\neg y_{j_{1}} \vee y_{j_{2}}\right)$, where $\varphi$ does not entail $\left(\neg y_{j_{2}} \vee y_{j_{1}}\right)$. To implement $\left(\neg x_{i} \vee x\right)$ we therefore identify $y_{j_{1}}$ with $x_{i}$ and $y_{j_{2}}$ with $x$. In the subsequent formula there are four implicative cases to consider:

1. $C=\left(\neg x \vee y_{j}\right)$,

2. $C=\left(\neg y_{j} \vee x\right)$,

3. $C=\left(\neg x_{i} \vee y_{j}\right)$,

4. $C=\left(\neg y_{j} \vee x_{i}\right)$,

where $y_{j}$ denotes a variable distinct from $x_{i}$ and $x$. If both the first and second case occurs simultaneously we identify $y_{j}$ with $x$. If only the first case occurs we identify $y_{j}$ with $c_{0}$, and if only the second case occurs we identify $y_{j}$ with $x_{i}$. If the third and four case occurs simultaneously we identify $y_{j}$ with $x_{i}$. If only the third case occurs we identify $y_{j}$ with $x$, and if only the fourth case occurs we identify $y_{j}$ with $c_{0}$. We then identify any remaining variable to $c_{0}$. Let $\varphi_{\rightarrow}\left(x, x_{i}, c_{0}\right)$ denote the resulting formula. Put together, we see that $R_{\mathrm{IS}}^{\kappa}\left(x_{1}, \ldots, x_{\kappa}, x, c_{0}\right) \equiv \varphi_{\mathrm{F}}\left(c_{0}\right) \wedge \varphi_{\mathrm{NAND}}\left(x_{1}, \ldots, x_{\kappa}, x, c_{0}\right) \wedge \varphi_{\rightarrow}\left(x, x_{1}, c_{0}\right) \wedge$ $\ldots \wedge \varphi_{\rightarrow}\left(x, x_{\kappa}, c_{0}\right)$. Again, it is not difficult to see that $R_{\mathrm{IS}} \mathrm{S}_{11}\left(x_{1}, \ldots, x_{\kappa}, x, c_{0}\right)$ is a base of $\mathrm{IS}_{11}^{\mathcal{K}}$, and that it is minimal in the sense of Definition 3.6. This concludes the proof.

\subsubsection{The Infinite Cases}

From the preceding section we now have a complete list of minimal weak bases for all Boolean co-clones of finite order. One might ask if similar results are not possible to obtain for the eight Boolean co-clones of infinite order. To prove such a result we cannot use Theorem 3.5 since a co-clone of infinite order by definition does not admit a finite core-size. In this section we instead consider a method to obtain a weak base of a co-clone of infinite order, based on determining the weak bases of a certain set of co-clones of finite order. We then leverage this result to provide weak bases for all Boolean co-clones of infinite order. 
Theorem 3.13. Let IC be a co-clone of infinite order over a finite domain and let $I C_{1}, I C_{2}, \ldots$ be an infinite chain of co-clones of finite order such that IC $=\bigcup_{i=1}^{\infty} I C_{i}$. Let $R_{\mathrm{IC}}$ denote the weak base of each $\mathrm{IC}_{i}$. Assume that $R_{\mathrm{IC}} \in\left\langle\left\{R_{\mathrm{IC}}{ }_{i+1}\right\}\right\rangle_{\exists}$ for each $i \geq 1$. Then the weak base of IC is the language $S_{\mathrm{IC}}=\left\{R_{\mathrm{IC}} \mid i \geq 1\right\}$.

Proof. First observe that each $\mathrm{IC}_{i}$ does indeed have a finite weak base since by assumption they are of finite order. To prove that $S_{\mathrm{IC}}$ is a weak base of IC we must prove that it is a base of IC and that $S_{\mathrm{IC}} \subseteq\langle S\rangle_{\exists}$ for each base $S$ of IC. It is easy to see that $S_{\mathrm{IC}}$ is a base of IC since $\mathrm{IC}_{i}=\left\langle\left\{R_{\mathrm{IC}}\right\}\right\rangle \subseteq\left\langle S_{\mathrm{IC}}\right\rangle$ for every $i \geq 1$. Let $S$ be a constraint language such that $\langle S\rangle=$ IC. Observe that $S$ must be infinite, and that there for every $R \in S$ exists some $i$ such that $R \in \mathrm{IC}_{i}$, since $\langle S\rangle=\mathrm{IC}=\bigcup_{i=1}^{\infty} \mathrm{IC}_{i}$.

We must prove that $\left\langle S_{I C}\right\rangle_{\nexists} \subseteq\langle S\rangle_{\exists}$. Let $R \in S_{I C}$ be a $\kappa$-ary relation. Then there exists a $\lambda$ such that $R$ is the weak base of $I C_{\lambda}$. We prove that there exists $S^{\prime} \subseteq S$ such that $\left\langle S^{\prime}\right\rangle=I C_{\lambda^{\prime}}$ for some $\lambda^{\prime} \geq \lambda$, since this implies that $R \in$ $\left\langle S^{\prime}\right\rangle_{\exists} \subseteq\langle S\rangle_{\exists}$, by the original assumption. Assume towards contradiction that no such set $S^{\prime}$ exists. But this implies that there exists some $\lambda^{\prime \prime}<\lambda$ such that $\langle S\rangle=I C_{\lambda^{\prime \prime}}$, which is clearly impossible since IC $\mathrm{I}_{\lambda^{\prime \prime}}$ is of finite order. Hence, there exists $S^{\prime} \subseteq S$ such that $R \in\left\langle S^{\prime}\right\rangle_{\exists}$. This in turn implies that $\left\langle S_{I C}\right\rangle_{\nexists} \subseteq\left\langle S^{\prime}\right\rangle_{\nexists}$, and that $S_{I C}$ is a weak base of IC.

We remark that since $R_{\mathrm{IC}} \in\left\langle\left\{R_{\mathrm{I}} C_{i+1}\right\}\right\rangle_{\exists}$ for every $i \geq 1$ we can in fact remove any finite number of relations from the weak base $S_{\mathrm{IC}}$ and still obtain a weak base of IC. Hence, even though $S_{\mathrm{IC}}$ will be minimal in the sense of Definition 3.6 whenever each $R_{\mathrm{IC}}$ is minimal, we see that this is not the only possible definition of minimality for infinite constraint languages.

According to Theorem 3.13 all that is needed to obtain weak bases for the eight co-clones of infinite order in the Boolean co-clone lattice, is to show that the condition $R_{\mathrm{IC}} \in\left\langle R_{\mathrm{IC}_{i+1}}\right\rangle_{\exists}$ is satisfied for every co-clone in $\left\{\mathrm{IS}_{0}^{i}, \mathrm{IS}_{02}^{i}, \mathrm{IS}_{01}^{i}, \mathrm{IS}_{00}^{i}, \mathrm{IS}_{1}^{i}, \mathrm{IS}_{12}^{i}, \mathrm{IS}_{11}^{i}, \mathrm{IS}_{10}^{i}\right\}$. We only consider the case $\mathrm{IS}_{00}^{\mathcal{K}}$ since the remaining proofs are entirely analogous. Hence, we need to show that the weak base of $1 \mathrm{~S}_{00}^{\mathcal{K}}$ can q.f.p.p. define the weak base of $\mathrm{IS}_{00}^{\mathcal{K}-1}$ for each $\kappa \geq 3$. For $\kappa \geq 2$ let $R_{\mathrm{IS}_{00}^{\kappa}}\left(x_{1}, \ldots, x_{\kappa}, x, c_{0}, c_{1}\right) \equiv \mathrm{OR}^{\kappa}\left(x_{1}, \ldots, x_{\kappa}\right) \wedge(x \rightarrow$ $\left.x_{1} \cdots x_{\kappa}\right) \wedge \mathrm{F}\left(c_{0}\right) \wedge \mathrm{T}\left(c_{1}\right)$. Then we can q.f.p.p. define $R_{\mathrm{IS}_{00}^{\kappa}}$ with $R_{\mathrm{IS}_{00}^{\kappa+1}}$ using the definition

$$
R_{\mathrm{IS}_{00}^{\kappa}}\left(x_{1}, \ldots, x_{\kappa}, x, c_{0}, c_{1}\right) \equiv R_{\mathrm{IS}_{00}^{\kappa+1}}\left(x_{1}, \ldots, x_{\kappa}, x_{\kappa}, x, c_{0}, c_{1}\right) .
$$

Hence, we obtain the following corollary.

Corollary 3.14. The following statements hold.

- $\left\{\mathrm{OR}^{\kappa}\left(x_{1}, \ldots, x_{\kappa}\right) \wedge \mathrm{T}\left(c_{1}\right) \mid \kappa \geq 2\right\}$ is a minimal weak base of $\mathrm{IS}_{0}$,

- $\left\{\mathrm{OR}^{\kappa}\left(x_{1}, \ldots, x_{\kappa}\right) \wedge \mathrm{F}\left(c_{0}\right) \wedge \mathrm{T}\left(c_{1}\right) \mid \kappa \geq 2\right\}$ is a minimal weak base of $\mathrm{IS}_{02}$,

- $\left\{\mathrm{OR}^{\kappa}\left(x_{1}, \ldots, x_{\kappa}\right) \wedge\left(x \rightarrow x_{1} \cdots x_{\kappa}\right) \wedge \mathrm{T}\left(c_{1}\right) \mid \kappa \geq 2\right\}$ is a minimal weak base of $\mathrm{IS}_{01}$, 
- $\left\{\mathrm{OR}^{\kappa}\left(x_{1}, \ldots, x_{\kappa}\right) \wedge\left(x \rightarrow x_{1} \cdots x_{\kappa}\right) \wedge \mathrm{F}\left(c_{0}\right) \wedge \mathrm{T}\left(c_{1}\right) \mid \kappa \geq 2\right\}$ is a minimal weak base of $\mathrm{IS}_{00}$,

- $\left\{\operatorname{NAND}^{\kappa}\left(x_{1}, \ldots, x_{\kappa}\right) \wedge \mathrm{F}\left(c_{0}\right) \mid \kappa \geq 2\right\}$ is a minimal weak base of $\mathrm{IS}_{1}$,

- $\left\{\mathrm{NAND}^{\kappa}\left(x_{1}, \ldots, x_{\kappa}\right) \wedge \mathrm{F}\left(c_{0}\right) \wedge \mathrm{T}\left(c_{1}\right) \mid \kappa \geq 2\right\}$ is a minimal weak base of $\mathrm{IS}_{12}$

- $\left\{\operatorname{NAND}^{\kappa}\left(x_{1}, \ldots, x_{\kappa}\right) \wedge\left(x_{1} \rightarrow x\right) \wedge \ldots \wedge\left(x_{\kappa} \rightarrow x\right) \wedge \mathrm{F}\left(c_{0}\right) \mid \kappa \geq 2\right\}$ is a minimal weak base of $\mathrm{IS}_{11}$,

- $\left\{\mathrm{NAND}^{\kappa}\left(x_{1}, \ldots, x_{\kappa}\right) \wedge\left(x_{1} \rightarrow x\right) \wedge \ldots \wedge\left(x_{\kappa} \rightarrow x\right) \wedge \mathrm{F}\left(c_{0}\right) \wedge \mathrm{T}\left(c_{1}\right) \mid \kappa \geq\right.$ $2\}$ is a minimal weak base of $\mathrm{IS}_{10}$.

Combining Lemma 3.8. Lemma 3.10. Lemma 3.11. Lemma 3.12 and Corollary 3.14 , we have thus proved the main result of this chapter.

Theorem 3.15. The relations in Table 3.1 are minimal weak bases.

\subsection{Summary and Conclusions}

We have used Schnoor's and Schnoor's technique for obtaining weak bases [SS08] and applied it in the Boolean domain. Our hope is that the relatively straightforward definitions in Table 3.1 are more accessible than manually needing to calculate relations of the form $\mathrm{C}\left(U^{c}\right)$ (which are also not guaranteed to be minimal). In particular, our weak bases for the eight infinite chains in the Boolean co-clone lattice are much simpler than the relations obtained via Schnoor's and Schnoor's procedure. In the process, we have also extended Schnoor's and Schnoor's technique for obtaining weak bases to co-clones of infinite order, and provided weak bases for the eight Boolean co-clones of infinite order. 


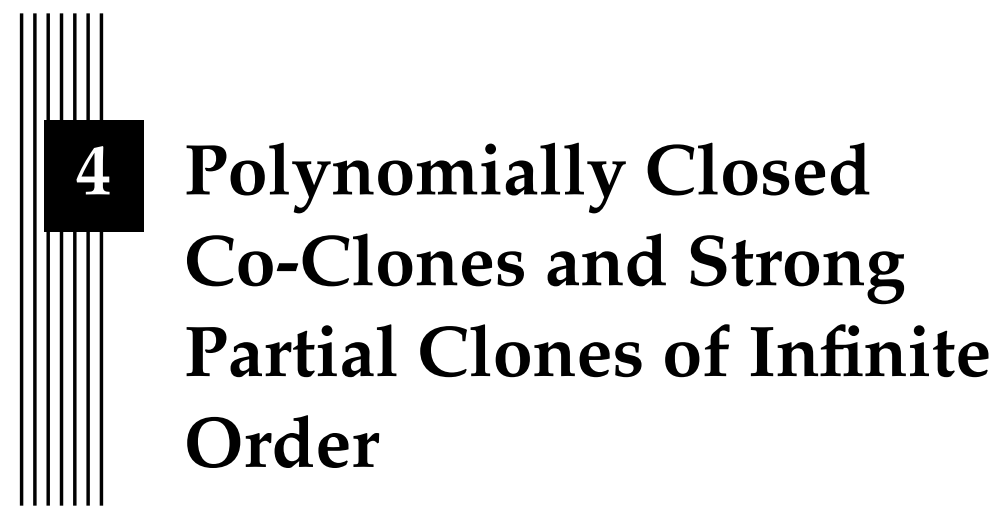

- Will you and take a walk with me? You hear the bird's gurgling?

- Which pleasure! which charm!

English as she is spoke

In this chapter we will apply the laborous work in Chapter 3 to study properties of co-clones. Roughly, the question we will study is the following: given a co-clone and a weak base of this co-clone, can we obtain an upper bound on the number of existentially quantified variables required to implement any relation in the co-clone? Using this question as a starting point we then proceed to show that many of the strong partial clones of interest in this thesis are of infinite order, and cannot be generated by a finite set of partial functions.

\subsection{Introduction}

Given a weak base $R_{\mathrm{IC}}$ of a co-clone $\operatorname{Inv}(\mathrm{C})$, how many existentially quantified variables are required to implement all other relations in the co-clone? Clearly, unless the interval of all strong partial clones corresponding to $\mathrm{C}$ consists of just one element, we know that at least one existentially quantified variable is required. More generally, we are interested in which $\kappa$-ary relations that can be p.p. defined with $1,2, \ldots, p(\kappa)$ existentially quantified variables, for some reasonably slowly growing function $p$. In the sequel we assume that $p$ is a polynomial function. If $p(\kappa)$ variables are sufficient to im- 
plement every $\kappa$-ary relation $R$ in a co-clone then we say that the co-clone is polynomially closed. We remark that if if $p(\kappa) \leq 2$ then the resulting set of definable relations over some language $S$ is similar to the closure operator considered in Nordh and Zanuttini [NZ09].

The first contribution of this chapter is a complete classification of the polynomially closed Boolean co-clones (in Section 4.3). Our proofs are based on comparing the least expressive language in the co-clone with the most expressive language in the co-clone, in order to obtain an upper bound of $p$. The least expressive language in a co-clone in this context is nothing else than a weak base of the co-clone, as studied in the previous chapter. The most expressive language in a co-clone is called a plain base, and were introduces by Creignou et al. |CKZ08|. We first give a general result and provide a sufficient condition for a co-clone over any finite domain to be polynomially closed: a co-clone $\operatorname{Inv}(\mathrm{C})$ is polynomially closed if $\mathrm{C}$ contains a k-ary near-unanimity function for some $k \geq 3$. We then complete this classification for the Boolean domain and in addition prove that a Boolean co-clone $\operatorname{Inv}(\mathrm{C})$ is polynomially closed if the functions in $\mathrm{C}$ can be represented by affine functions, or if $\operatorname{Inv}(C)$ is of infinite order. To handle the last case we make use of the weak bases of infinite order provided by Corollary 3.14 from the previous chapter. In Section 4.5 we then proceed with the problem of determining whether a co-clone is superpolynomially closed. We first prove that if the number of $\kappa$-ary relations in a co-clone is sufficiently large, then, for any finite base of the co-clone, there exists relations which cannot be p.p. defined using a polynomial number of existentially quantified variables. By using a result of Berman et al. [BMMVW06] we then obtain a sufficient condition for verifying whether a co-clone over any finite domain is superpolynomially closed. We remark that for the Boolean domain, a co-clone of finite order is polynomially closed if and only if the corresponding clone contains a $k$-edge function for some $k \geq 2$, or, equivalently, if the clone has few subpowers [BMMVW06]. Interestingly, this does not hold for co-clones of infinite order, which suggests a quantitative difference between our notion and that of Berman et al.

The second contribution of this chapter (in Section 4.6) is an investigation of the structure of the partial polymorphisms of finite constraint languages corresponding to superpolynomially closed co-clones. Consider e.g. the relation $R_{1 / 3}=\{(0,0,1),(0,1,0),(1,0,0)\}$ from Chapter 2 and recall that $\left\langle\left\{R_{1 / 3}\right\}\right\rangle=\mathrm{BR}$. Since this relation only consists of three tuples and have a very simple structure, one might believe that describing its partial polymorphisms is a rather straightforward task. Would it e.g. be possible to find a finite base of $\operatorname{pPol}\left(\left\{R_{1 / 3}\right\}\right)$ ? We prove that such a finite base cannot exist (irregardless of any complexity theoretical assumptions) since BR according to the results in Section 4.5 is superpolynomially closed. In fact, we prove something stronger: let $S$ be a finite constraint language over an arbitrary finite domain. If the co-clone of $S$ is superpolynomially closed, and if the polymorphims of $S$ are essentially unary, then the set of partial polymorphisms of $S$ is of infinite order. This result can be seen as a continuation of the research by Haddad and Börner [BH98] who gave a condition for checking 
whether a strong partial clone is infinitely generated, but, as will be evident in Chapter 7, our result also has many practical consequences for the applicability of partial clone theory to the study of the computational complexity of NP-complete CSP $(\cdot)$ problems.

\subsection{Preliminaries}

Recall from Chapter 3 that $\operatorname{Int}_{[\cdot]_{s}}(\mathrm{C})$ denotes the set of all strong partial clones whose total component equals the clone $C$, and that a weak base $S_{w}$ of $\operatorname{Inv}(\mathrm{C})$ is a base with the property that $\operatorname{pPol}\left(S_{w}\right)$ is the largest element of $\operatorname{Int}_{[\cdot]_{s}}(\mathrm{C})$. Clearly, the smallest element in $\operatorname{Int}_{[\cdot]_{s}}(C)$ is simply the strong partial clone obtained by closing $C$ under subfunctions. From the relational point of view, this is equivalent to the fact that $\langle\operatorname{Inv}(C)\rangle_{\exists}=\operatorname{Inv}(C)$. More generally, given a co-clone $\operatorname{Inv}(\mathrm{C})$ we call a constraint language $S_{\mathrm{pl}}$ such that $\left\langle S_{\mathrm{pl}}\right\rangle_{\nexists}=\operatorname{Inv}(\mathrm{C})$ a plain base of $\operatorname{Inv}(\mathrm{C})$. The problem of finding more succinct plain bases for Boolean co-clones was considered in Creignou et al. |CKZ08|. These plain bases are summarised in Table 4.1. where the relation $\operatorname{Compl}_{\lambda, \kappa}$ is defined as

$$
\begin{aligned}
\operatorname{Compl}_{\lambda, \kappa} & \left(x_{1}, \ldots, x_{\lambda+\kappa}\right) \equiv \\
& \left(x_{1} \vee \ldots \vee x_{\kappa} \vee \overline{x_{\kappa+1}} \vee \ldots \vee \overline{x_{\kappa+\lambda}}\right) \wedge \\
& \left(\overline{x_{1}} \vee \ldots \vee \overline{x_{\kappa}} \vee x_{\kappa+1} \vee \ldots \vee x_{\kappa+\lambda}\right) .
\end{aligned}
$$

Observe that the plain bases of $\mathrm{IN}_{2}$ and BR in Table 4.1 are equivalent to $\bigcup_{\kappa=1}^{\infty} \Gamma_{\mathrm{NAE}}^{\kappa}$ and $\bigcup_{\kappa=1}^{\infty} \Gamma_{\mathrm{SAT}}^{\kappa}$.

Hence, a plain base $S_{\mathrm{pl}}$ of a co-clone $\operatorname{Inv}(\mathrm{C})$ is a base of $\operatorname{Inv}(\mathrm{C})$ that satisfies $\operatorname{pPol}\left(S_{\mathrm{pl}}\right) \subseteq \operatorname{pPol}(S)$ for any $\operatorname{pPol}(S) \in \operatorname{Int}_{[\cdot]_{s}}(\mathrm{C})$, and from the relational point of view a base of $\operatorname{Inv}(\mathrm{C})$ that satisfies $\left\langle S_{\mathrm{pl}}\right\rangle_{\nexists} \supseteq\langle S\rangle_{\exists}$ for any $\operatorname{pPol}(S) \in \operatorname{Int}_{[\cdot]_{S}}(\mathrm{C})$. We therefore have the following straightforward theorem.

Theorem 4.1. Let $S_{\mathrm{pl}}$ be a plain base of a co-clone $\operatorname{Inv}(\mathrm{C})$. Then $R \in\langle S\rangle_{\exists}$ for any $R \in \operatorname{Inv}(\mathrm{C})$.

It is not difficult to verify that each plain base $S_{\mathrm{pl}}$ in Table 4.1 in addition satisfies the slightly stronger condition that $R$ can be q.f.p.p. defined in $S_{\mathrm{pl}}$ using relations from $S_{\mathrm{pl}}$ of arity at most $\operatorname{ar}(R)$. As a shorthand, we let $S^{(\leq \kappa)}=$ $\{R \in S \mid \operatorname{ar}(R) \leq \kappa\}$ for any language $S$. Hence, for Boolean co-clones, Theorem 4.1 can be strengthened to the following.

Theorem 4.2. Let $S_{\mathrm{pl}}$ be the plain base from Table 4.1 for some Boolean co-clone $\operatorname{Inv}(\mathrm{C})$. Then $R \in\left\langle S_{\mathrm{pl}}^{(\leq \kappa)}\right\rangle_{\exists}$ for any $\kappa$-ary $R \in \operatorname{Inv}(\mathrm{C})$.

\subsection{Polynomially Closed Co-Clones of Finite Order}

In this section we formally introduce the notion of a polynomially closed co-clone. Intuitively, the notion means that for any base of the co-clone, a 
Table 4.1: Plain bases of all Boolean co-clones.

\begin{tabular}{|c|c|}
\hline Co-clone & Plain base \\
\hline IBF & $\left\{\mathrm{Eq}\left(x_{1}, x_{2}\right)\right\}$ \\
\hline $\mathrm{IR}_{0}$ & $\left\{\mathrm{~F}\left(c_{0}\right)\right\}$ \\
\hline $\mathrm{IR}_{1}$ & $\left\{\mathrm{~T}\left(c_{1}\right)\right\}$ \\
\hline $\mathrm{IR}_{2}$ & $\left\{\mathrm{~F}\left(c_{0}\right), \mathrm{T}\left(c_{1}\right)\right\}$ \\
\hline IM & $\left\{\left(x_{1} \rightarrow x_{2}\right)\right\}$ \\
\hline $\mathrm{IM}_{0}$ & $\left\{\left(x_{1} \rightarrow x_{2}\right), \mathrm{F}\left(c_{0}\right)\right\}$ \\
\hline $\mathrm{IM}_{1}$ & $\left\{\left(x_{1} \rightarrow x_{2}\right), \mathrm{T}\left(c_{1}\right)\right\}$ \\
\hline $\mathrm{IM}_{2}$ & $\left\{\left(x_{1} \rightarrow x_{2}\right), \mathrm{F}\left(c_{0}\right), \mathrm{T}\left(c_{1}\right)\right\}$ \\
\hline $\mathrm{IS}_{0}^{\kappa}, \kappa \geq 2$ & $\left\{\mathrm{OR}^{\kappa}\left(x_{1}, \ldots, x_{\kappa}\right)\right\}$ \\
\hline $\mathrm{IS}_{0}-$ & $\left\{\mathrm{OR}^{\kappa}\left(x_{1}, \ldots, x_{\kappa}\right) \mid \kappa \geq 1\right\}$ \\
\hline $\mathrm{IS}_{02}^{\kappa}, \kappa \geq 2$ & $\left\{\mathrm{OR}^{\kappa}\left(x_{1}, \ldots, x_{\kappa}\right), \mathrm{F}\left(c_{0}\right)\right\}$ \\
\hline $\mathrm{IS}_{02}$ & $\left\{\mathrm{OR}^{\kappa}\left(x_{1}, \ldots, x_{\kappa}\right) \mid \kappa \geq 1\right\} \cup\left\{\mathrm{F}\left(c_{0}\right)\right\}$ \\
\hline $\mathrm{IS}_{01}^{\kappa}, \kappa \geq 2$ & $\left\{\mathrm{OR}^{\kappa}\left(x_{1}, \ldots, x_{\kappa}\right),\left(x_{1} \rightarrow x_{2}\right)\right\}$ \\
\hline $\mathrm{IS}_{01}-\mathrm{C}$ & $\left\{\mathrm{OR}^{\kappa}\left(x_{1}, \ldots, x_{\kappa}\right) \mid \kappa \geq 1\right\} \cup\left\{\left(x_{1} \rightarrow x_{2}\right)\right\}$ \\
\hline$I S_{00}^{\kappa}, \kappa \geq 2$ & $\left\{\mathrm{OR}^{\kappa}\left(x_{1}, \ldots, x_{\kappa}\right),\left(x_{1} \rightarrow x_{2}\right), \mathrm{F}\left(c_{0}\right)\right\}$ \\
\hline$I S_{00}$ & $\left\{\mathrm{OR}^{\kappa}\left(x_{1}, \ldots, x_{\kappa}\right) \mid \kappa \geq 1\right\} \cup\left\{\left(x_{1} \rightarrow x_{2}\right), \mathrm{F}\left(c_{0}\right)\right\}$ \\
\hline$I S_{1}^{\kappa}, \kappa \geq 2$ & $\left\{\operatorname{NAND}^{\kappa}\left(x_{1}, \ldots, x_{\kappa}\right)\right\}$ \\
\hline & $\left\{\operatorname{NAND}^{\kappa}\left(x_{1}, \ldots, x_{\kappa}\right) \mid \kappa \geq 1\right\}$ \\
\hline $\mathrm{IS}_{12}^{\kappa}, \kappa \geq 2$ & $\left\{\operatorname{NAND}^{\kappa}\left(x_{1}, \ldots, x_{\kappa}\right), \mathrm{T}\left(\overline{c_{1}}\right)\right\}$ \\
\hline $\mathrm{IS}_{12}$ & $\left\{\operatorname{NAND}^{\kappa}\left(x_{1}, \ldots, x_{\kappa}\right) \mid \kappa \geq 1\right\} \cup\left\{\mathrm{T}\left(c_{1}\right)\right\}$ \\
\hline$I S_{11}^{\kappa}, \kappa \geq 2$ & $\left\{\operatorname{NAND}^{\kappa}\left(x_{1}, \ldots, x_{\kappa}\right),\left(x_{1} \rightarrow x_{2}\right)\right\}$ \\
\hline $\mathrm{IS}_{11}$ & $\left\{\operatorname{NAND}^{\kappa}\left(x_{1}, \ldots, x_{\kappa}\right)\right\} \cup\left\{\left(x_{1} \rightarrow x_{2}\right)\right\}$ \\
\hline $\mathrm{IS}_{1}^{\kappa}, \kappa \geq 2$ & $\left\{\operatorname{NAND}^{\kappa}\left(x_{1}, \ldots, x_{\kappa}\right),\left(x_{1} \rightarrow x_{2}\right), \mathrm{T}\left(c_{1}\right)\right\}$ \\
\hline $\mathrm{IS}_{10}-$ & $\left\{\operatorname{NAND}^{\kappa}\left(x_{1}, \ldots, x_{\kappa}\right) \mid \kappa \geq 1\right\} \cup\left\{\left(x_{1} \rightarrow x_{2}\right), \mathrm{T}\left(c_{1}\right)\right\}$ \\
\hline ID & $\left\{\left(x_{1} \oplus x_{2}=1\right)\right\}$ \\
\hline $\mathrm{ID}_{1}$ & $\left\{\left(x_{1} \oplus x_{2}=1\right)\right\} \cup\left\{\mathrm{F}\left(c_{0}\right), \mathrm{T}\left(c_{1}\right)\right\}$ \\
\hline $\mathrm{ID}_{2}$ & $\left\{\mathrm{~F}\left(c_{0}\right), \mathrm{T}\left(c_{1}\right),\left(x_{1} \vee x_{2}\right),\left(\neg x_{1} \vee x_{2}\right),\left(\neg x_{1} \vee \neg x_{2}\right)\right\}$ \\
\hline IL & $\left\{\left(x_{1} \oplus \ldots \oplus x_{\kappa}=0\right) \mid \kappa\right.$ even $\}$ \\
\hline $\mathrm{IL}_{0}$ & $\left\{\left(x_{1} \oplus \ldots \oplus x_{\kappa}=0\right) \mid \kappa \in \mathbb{N}\right\}$ \\
\hline $\mathrm{IL}_{1}$ & $\left\{\left(x_{1} \oplus \ldots \oplus x_{\kappa}=c\right) \mid \kappa \in \mathbb{N}, c=\kappa \bmod 2\right\}$ \\
\hline $\mathrm{IL}_{2}$ & $\left\{\left(x_{1} \oplus \ldots \oplus x_{\kappa}=c\right) \mid \kappa \in \mathbb{N}, c \in\{0,1\}\right\}$ \\
\hline $\mathrm{IL}_{3}$ & $\left\{\left(x_{1} \oplus \ldots \oplus x_{\kappa}=c\right) \mid \kappa\right.$ even,$\left.c \in\{0,1\}\right\}$ \\
\hline IV & $\left\{\left(x_{1} \vee \ldots \vee x_{\kappa} \vee \neg x\right) \mid \kappa \geq 1\right\}$ \\
\hline $\mathrm{IV}_{0}$ & $\left\{\left(x_{1} \vee \ldots \vee x_{\kappa} \vee \neg x\right) \mid \kappa \bar{\in} \mathbb{N}\right\}$ \\
\hline $\mathrm{IV}_{1}$ & $\left.\left\{\mathrm{OR}^{\kappa}\left(x_{1}, \ldots, x_{\kappa}\right) \mid \kappa \in \mathbb{N}\right\} \cup\left\{\left(x_{1} \vee \ldots \vee x_{\kappa} \vee \neg x\right) \mid \kappa \geq 1\right\}\right)$ \\
\hline $\mathrm{IV}_{2}$ & $\left.\left\{\mathrm{OR}^{\kappa}\left(x_{1}, \ldots, x_{\kappa}\right) \mid \kappa \in \mathbb{N}\right\} \cup\left\{\left(x_{1} \vee \ldots \vee x_{\kappa} \vee \neg x\right) \mid \kappa \in \bar{N}\right\}\right)$ \\
\hline IE & $\left\{\left(\neg x_{1} \vee \ldots \vee \neg x_{\kappa} \vee x\right) \mid \kappa \geq 1\right\}$ \\
\hline $\mathrm{IE}_{0}$ & $\left\{\left(\neg x_{1} \vee \ldots \vee \neg x_{\kappa} \vee x\right) \mid \kappa \in \mathbb{N}\right\}$ \\
\hline $\mathrm{IE}_{1}$ & $\left.\left\{\operatorname{NAND}^{\kappa}\left(x_{1}, \ldots, x_{\kappa}\right) \mid \kappa \in \mathbb{N}\right\} \cup\left\{\left(\neg x_{1} \vee \ldots \vee \neg x_{\kappa} \vee x\right) \mid \kappa \geq 1\right\}\right)$ \\
\hline $\mathrm{IE}_{2}$ & $\left.\left\{\operatorname{NAND}^{\kappa}\left(x_{1}, \ldots, x_{\kappa}\right) \mid \kappa \in \mathbb{N}\right\} \cup\left\{\left(\neg x_{1} \vee \ldots \vee \neg x_{\kappa} \vee x\right) \mid \kappa \in \mathbb{N}\right\}\right)$ \\
\hline IN & $\left\{\operatorname{Compl}_{\lambda,} \mid \lambda, \kappa \geq 1\right\}$ \\
\hline $\mathrm{IN}_{2}$ & $\left\{\right.$ Compl $\left._{\lambda,} \mid \lambda, \kappa \in \mathbb{N}\right\}$ \\
\hline II & $\left\{\left(x_{1} \vee \ldots \vee x_{\lambda} \vee \neg y_{1} \vee \ldots \neg y_{\kappa}\right) \mid \lambda, \kappa \geq 1\right\}$ \\
\hline $\mathrm{II}_{0}$ & $\left\{\left(x_{1} \vee \ldots \vee x_{\lambda} \vee \neg y_{1} \vee \ldots \neg y_{\kappa}\right) \mid \lambda \in \mathbb{N}, \kappa \geq 1\right\}$ \\
\hline $\mathrm{II}_{1}$ & $\left\{\left(x_{1} \vee \ldots \vee x_{\lambda} \vee \neg y_{1} \vee \ldots \neg y_{\kappa}\right) \mid \lambda \geq 1, \kappa \in \mathbb{N}\right\}$ \\
\hline BR & $\left\{\left(x_{1} \vee \ldots \vee x_{\lambda} \vee \neg y_{1} \vee \ldots \neg y_{\kappa}\right) \mid \lambda, \kappa \in \mathbb{N}\right\}$ \\
\hline
\end{tabular}


polynomial amount of variables is sufficient to p.p. implement any relation in the co-clone.

Definition 4.3. Let $\operatorname{Inv}(\mathrm{C})$ be a co-clone over a finite domain. We say that $\operatorname{Inv}(\mathrm{C})$ is polynomially closed if there exists a polynomial $p$ such that for every base $S$ of $\operatorname{Inv}(\mathrm{C})$ and every $\kappa$-ary $R \in \operatorname{Inv}(\mathrm{C})$ it holds that $R$ can be p.p. defined in $S$ with at most $p(\kappa)$ existentially quantified variables.

Observe that $\operatorname{Inv}(\mathrm{C})$ in this definition is allowed to be of infinite order. In this section, however, we restrict our focus to co-clones of finite order, while we in Section 4.4 investigate co-clones of infinite order. If a co-clone is not polynomially closed then we say that it is superpolynomially closed. See Figure 4.3 for a visualisation of the Boolean co-clones that are polynomially respectively superpolynomially closed. That the classification in Figure 4.3 is correct is proved in Theorem 4.9 later in this section, Theorem 4.10 in Section 4.4 and Theorem 4.15 in Section 4.5 .

In order to prove that a co-clone is polynomially closed it is sufficient to prove that there exists some polynomial $p$ such that the weak base of the coclone can implement any $\kappa$-ary relation with $p(\kappa)$ variables. A useful method to accomplish this is to first prove that every relation in the co-clone can be expressed as a q.f.p.p. definition over the plain base with a polynomial number of constraints. Formally, we say that a plain base $S$ of a co-clone $\operatorname{Inv}(C)$ is a polynomial base if there exists a polynomial $p$, such that every $\kappa-$ ary $R \in \operatorname{Inv}(\mathrm{C})$ has a q.f.p.p. implementation over $S^{(\leq \kappa)}$, with at most $p(\kappa)$ constraints. Polynomial bases and polynomially closed co-clones are related by the following lemma, which states that a polynomial base for a co-clone implies polynomial closure, under some additional conditions.

Lemma 4.4. Let $\operatorname{Inv}(\mathrm{C})$ be a co-clone with a weak base $R_{w}$. If there exists a polynomial, plain base $S_{\mathrm{pl}}$ of $\operatorname{Inv}(\mathrm{C})$, and a polynomial $p$ such that $R_{w}$ can p.p. define every relation in $S_{\mathrm{pl}}^{(\leq \kappa)}$ with at most $p(\kappa)$ existentially quantified variables, then $\operatorname{Inv}(\mathrm{C})$ is polynomially closed.

Proof. Let $R \in \operatorname{Inv}(\mathrm{C})$ be a $\kappa$-ary relation. By Theorem 4.1 and the assumption that $S_{\mathrm{pl}}$ is a polynomial, plain base it follows that $S_{\mathrm{pl}}^{(\kappa)}$ can q.f.p.p. implement $R$ using at most $g(\kappa)$ constraints for some polynomial $g$. Let $\phi$ denote the q.f.p.p. implementation of $R$ in $S_{\mathrm{pl}}^{(\leq \kappa)}$. For every constraint $C_{i}$ in $\phi$ we then replace $C_{i}$ with its p.p. implementation over $\left\{R_{w}\right\}$. Let the resulting formula be $\phi^{\prime}$. Since $\phi$ had $g(\kappa)$ constraints and each constraint in $\phi^{\prime}$ introduced at most $p(\kappa)$ new existentially quantified variables, the total number of variables in $\phi^{\prime}$ is $g(\kappa) \cdot p(\kappa)$, clearly polynomial with respect to $\kappa$. Hence, $\operatorname{Inv}(\mathrm{C})$ is polynomially closed.

It is not difficult to see that this condition is satisfied whenever a co-clone has a finite plain base.

Lemma 4.5. If $\operatorname{Inv}(C)$ has a finite plain base then $\operatorname{Inv}(C)$ is polynomially closed. 


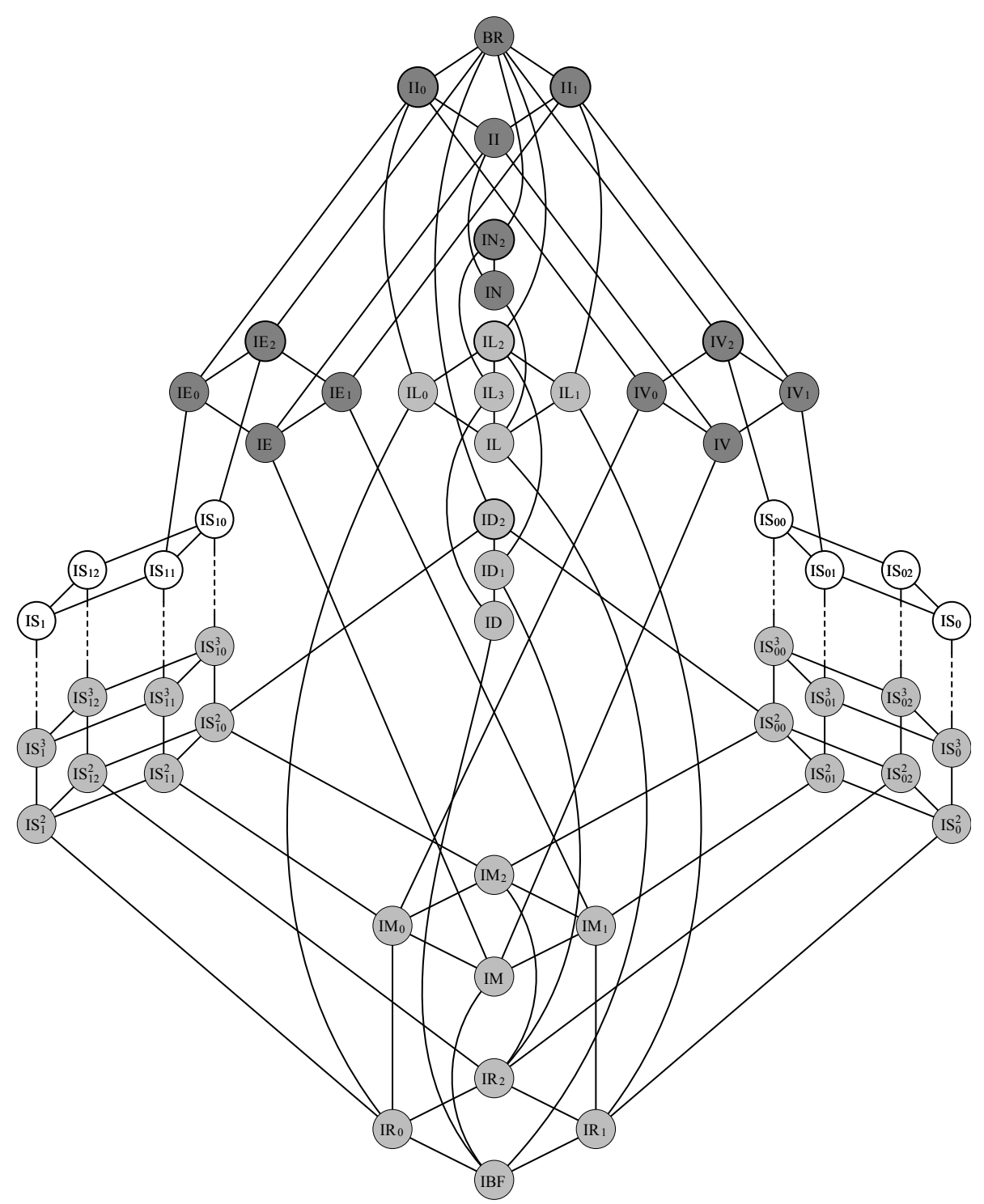

Figure 4.1: The lattice of Boolean co-clones. The co-clones of finite order which are polynomially closed are coloured in grey. The co-clones of infinite order that are polynomially closed are coloured in white. The superpolynomially closed co-clones are coloured in dark grey. 
Proof. Assume that $\operatorname{Inv}(\mathrm{C})$ has a plain base $S_{\mathrm{pl}}$ of finite cardinality and let $R_{w}$ denote a weak base of $\operatorname{Inv}(\mathrm{C})$. Observe that $S_{\mathrm{pl}}$ is trivially a polynomial base. Since $S_{\mathrm{pl}}$ is finite there exists a polynomial $p$ such that $R_{w}$ can p.p. implement $S_{\mathrm{pl}}^{(\leq \kappa)}$ for every $\kappa \geq 1$ with $p(\kappa)$ variables. To see this, simply take the number of existentially quantified variables of the relation requiring the largest number of quantified variables in the p.p. definition in $S_{\mathrm{pl}}$. Such a relation must exist since $S_{\mathrm{pl}}$ is finite. The result then follows from Lemma 4.4

For the Boolean domain one can simply consult Table 4.1 to see which co-clones have finite plain bases. It is however possible to give a general characterisation of the co-clones admitting finite plain bases. Let $D$ be an arbitrary finite domain. A n-ary near-unanimity (NU) operation on $D$ for $n \geq$ 3 is an operation $f: D^{n} \rightarrow D$ such that $f(x, y, \ldots, y)=f(y, x, \ldots, y)=\ldots=$ $f(y, y, \ldots, x)=y$ for all $x, y \in D$ (we may note that this is a special case of an $n$-edge operation, used in Section 4.5).

Theorem 4.6. Let $\operatorname{Inv}(\mathrm{C})$ be a co-clone over a finite domain $D$ such that $C$ contains an $n$-ary NU operation for some $n \geq 3$. Then $\operatorname{Inv}(\mathrm{C})$ is polynomially closed.

Proof. We recall some definitions from Jeavons et al. |[JCC98|. Let $R \subseteq D^{\kappa}$ be a relation and $I=\left\{i_{1}, \ldots, i_{d}\right\}$ a set of indices, $1 \leq i_{1}<\ldots<i_{d} \leq \kappa$. The projection of $R$ onto $I$ is the relation $\pi_{I}(R)=\left\{\left(t\left[i_{1}\right], \ldots, t\left[i_{d}\right]\right) \mid t \in R\right\}$. A relation $R \subseteq D^{\kappa}$ over $D$ is $r$-decomposable if it is equivalent to the conjunction of all its projections of arity at most $r$, i.e., for every $t \in\left(D^{\kappa} \backslash R\right)$ there is a set $I=\left\{i_{1}, \ldots, i_{d}\right\}$ as above such that $\left(t\left[i_{1}\right], \ldots, t\left[i_{d}\right]\right) \notin \pi_{I}(R)$. It is known that any relation preserved by an $n$-ary, $n \geq 3$, NU operation is $(n-1)$ decomposable [JCC98].

Now let $R \in \operatorname{Inv}(\mathrm{C})$ be a $\kappa$-ary relation. Observe that $\pi_{I}(R)$ can be defined using existential quantification over $R$, hence $\pi_{I}(R) \in\langle\{R\}\rangle$ for every set of indices $I$. Also, note that $\bigwedge_{I:|I|<n} \pi_{I}(R)$ is a q.f.p.p. implementation of $R$. Hence, the set of all relations $R^{\prime} \in \operatorname{Inv}(\mathrm{C})$ of arity at most $n-1$ is a plain base of $\operatorname{Inv}(C)$. Clearly, this is a finite set (since $D$ is finite). Thus, $\operatorname{Inv}(C)$ is polynomially closed by Lemma 4.5 .

Observe, however, that Lemma 4.5 or Theorem 4.6 are not applicable for the co-clones $\mathrm{IL}, \mathrm{IL}_{0}, \mathrm{IL}_{1}, \mathrm{IL}_{3}$ and $\mathrm{IL}_{2}$ since they do not admit finite plain bases. Fortunately, it is rather straightforward to prove that these co-clones admit polynomial bases, since the included relations can be viewed as linear equations over the field $\mathrm{GF}(2)$.

Lemma 4.7. $\mathrm{IL}, \mathrm{IL}_{0}, \mathrm{IL}_{1}, \mathrm{IL}_{3}$ and $\mathrm{IL}_{2}$ have polynomial, plain bases.

Proof. We only consider $\mathrm{IL}_{2}$ since the other cases follow through similar arguments. Every $\kappa$-ary relation $R \in \mathrm{IL}_{2}$ can according to Theorem 4.2 be expressed by an $S_{\mathrm{pl}}^{(\leq \kappa)}$-formula $\phi$ with $\mu$ constraints, where $S_{\mathrm{pl}}$ is the plain base of $\mathrm{IL}_{2}$ in Table 4.1 Thus, every constraint $C_{i}$ in $\phi$ is of the form 
$\left(x_{i_{1}} \oplus \ldots \oplus x_{i_{\kappa}}\right)=c_{i}$, where $c_{i} \in\{0,1\}$. Create an $\mu \times(\kappa+1)$-matrix $M$ such that each entry $r_{i, j}, 1 \leq j \leq \kappa$, is equal to 1 if the variable $x_{j}$ is included in the constraint $C_{i}$, and 0 otherwise. The entry $r_{i, k+1}$ is equal to the constant $c_{i}$ in $C_{i}$. Then it is not hard to verify that if the row $r_{i+1}$ is linearly dependent on $r_{1}, \ldots, r_{i}$ then $C_{1}, \ldots C_{i}$ entails $C_{i+1}$ in any satisfying assignment. Hence, we only need to keep the rows that are linearly independent, which gives the bound $\min (\kappa+1, \mu)$ on the number of constraints.

Lemma 4.8. $\mathrm{IL}, \mathrm{IL}_{0}, \mathrm{IL}_{1}, \mathrm{IL}_{3}$ and $\mathrm{IL}_{2}$ are polynomially closed.

Proof. We only present the proof of $\mathrm{IL}_{2}$ since the other co-clones follow through entirely analogous arguments. Let $S_{\mathrm{pl}}$ and $R_{w}$ be the plain and weak base of $\mathrm{IL}_{2}$ from Table 4.1. respectively. Since $\mathrm{IL}_{2}$ has a polynomial base by Lemma 4.7 all we need to prove is that there exists a polynomial $p$ such that $R_{w}$ can p.p. define $S_{\mathrm{pl}}^{(\leq \kappa)}$ using at most $p(\kappa)$ existentially quantified variables. We first and most crucially show that $S_{\mathrm{pl}}^{(\leq \kappa)}$ can implement $S_{\mathrm{pl}}^{(\leq \kappa+1)}$ with only one extra variable, for every $\kappa \geq 3$, with the implementation

$$
\begin{gathered}
\left(x_{1} \oplus \ldots \oplus x_{\kappa+1}=c\right) \equiv \exists x .\left(x_{1} \oplus \ldots \oplus x_{\kappa-1} \oplus x=c\right) \wedge \\
\left(x_{\kappa} \oplus x_{\kappa+1} \oplus x=0\right) .
\end{gathered}
$$

In addition to one quantified variable this requires one extra $S_{\mathrm{pl}}^{(\leq 3)}$-constraint. Hence, if $3 \leq \kappa \leq \lambda$ then $S_{\mathrm{pl}}^{(\leq \kappa)}$ can implement every relation in $S_{\mathrm{pl}}^{(\leq \lambda)}$ with $O(\lambda-\kappa)$ variables and $\lambda-\kappa$ additional $S_{\mathrm{pl}}^{(\leq 3)}$-constraints. By this it first follows that $S_{\mathrm{pl}}^{(\leq 3)}$ can p.p. define any relation in $S_{\mathrm{pl}}^{(\leq \kappa)}$ with at most $\kappa-3$ variables and $\kappa-2$ constraints. The weak base $R_{w}$ can then p.p. implement $S_{\mathrm{pl}}^{(\leq 3)}$ with a fixed number of variables since the arity of each relation is bounded, for example we have that

$$
\left(x_{1} \oplus x_{2} \oplus x_{3}=0\right) \equiv \exists y_{1}, y_{2}, y_{3}, c_{0}, c_{1} . R_{w}\left(x_{1}, x_{2}, x_{3}, y_{1}, y_{2}, y_{3}, c_{0}, c_{1}\right)
$$

and

$$
\left(x_{1} \oplus x_{2} \oplus x_{3}=1\right) \equiv \exists y_{1}, y_{2}, y_{3}, c_{0}, c_{1} . R_{w}\left(y_{1}, y_{2}, y_{3}, x_{1}, x_{2}, x_{3}, c_{0}, c_{1}\right) .
$$

Put together, this implies that $R_{w}$ can p.p. define any $S_{\mathrm{pl}}^{(\leq \kappa)}$ with $O(\kappa)$ existentially quantified variables, and by Lemma 4.4 that $\mathrm{IL}_{2}$ is polynomially closed.

Combining all results so far in this section, we obtain the following characterisation of the polynomially closed Boolean co-clones of finite order.

Theorem 4.9. If $\operatorname{Inv}(\mathrm{C}) \subseteq \operatorname{Inv}(\mathrm{X})$ for some $X \in\left\{\mathrm{L}_{2}, \mathrm{D}_{2}\right\} \cup\left\{\mathrm{S}_{00}^{\kappa}, \mathrm{S}_{10}^{\kappa} \mid \kappa \geq 2\right\}$ then $\operatorname{Inv}(\mathrm{C})$ is polynomially closed. 


\subsection{Polynomially Closed Co-Clones of Infinite Order}

So far we have only been concerned with polynomially closed co-clones of finite order. For co-clones of infinite order, we cannot use any of the machinery introduced in Section 4.3. In particular, Lemma 4.5 breaks down since there by definition cannot exist a finite plain base of a co-clone of infinite order. Fortunately, recall from Corollary 3.14 from Chapter 3 that we have simple, relational descriptions of the weak bases for all Boolean co-clones of infinite order. Hence, for every such co-clone $\operatorname{Inv}(C)$, we only have to show that there exists a polynomial $p$ such that the weak base can p.p. define every $\kappa$-ary relation in $R$ using at most $p(\kappa)$ existentially quantified variables.

Similarly to the proof of Lemma 3.11 we will exploit the fact that every relation in these co-clones can be written as restricted IHSB formulas that are prime |CKZ08|. Recall that $\mathrm{IHSB}_{+}^{\kappa}$ denotes the set of formulas of the form $\left(x_{1} \vee \ldots \vee x_{\mathcal{K}^{\prime}}\right), 1 \leq \kappa^{\prime} \leq \kappa,\left(\neg x_{1}\right),\left(\neg x_{1} \vee x_{2}\right)$, and dually for $\mathrm{IHSB}_{-}^{\kappa}$.

Theorem 4.10. $\mathrm{IS}_{0}, \mathrm{IS}_{02}, \mathrm{IS}_{01}, \mathrm{IS}_{00}, \mathrm{IS}_{1}, \mathrm{IS}_{12}, \mathrm{IS}_{11}$ and $\mathrm{IS}_{10}$ are polynomially closed.

Proof. We only consider $\mathrm{IS}_{00}$ since the other cases follow through similar arguments. Let $W_{\mathrm{IS}_{00}}=\left\{R_{\mathrm{IS}_{00}^{i}} \mid i \geq 2\right\}$ denote the weak base of $\mathrm{IS}_{00}$ from Table 3.1 in Chapter 3. We must prove that there exists a polynomial $p$ such that $W_{\mathrm{IS}_{00}}$ can p.p. define any $\kappa$-ary $R \in \mathrm{IS}_{00}$ using at most $p(\kappa)$ existentially quantified variables. Since $R \in \mathbb{I S}_{00}$ it is easily seen that there exists some $\kappa^{\prime} \geq 2$ such that $R \in \mathrm{IS}_{00}^{\kappa^{\prime}}$.

Hence, $R$ can be written as a prime $\operatorname{IHSB}_{+}^{\kappa^{\prime}}$ formula $\varphi$ over $x_{1}, \ldots, x_{\kappa}[\overline{C K Z 08}]$, and we need to show that it is possible to p.p. define this formula without requiring more than a polynomial number of existentially quantified variables. There are a few different cases to consider depending on the clauses of $\varphi$. Let $c_{0}$ and $c_{1}$ be two fresh variables distinct from $x_{1}, \ldots, x_{\kappa}$. First, we implement every clause in $\varphi$ of the form $\left(x_{i_{1}} \vee \ldots \vee x_{i_{j}}\right)$ for some $j \leq \kappa^{\prime}$ with the constraint $R_{\mathrm{IS}_{00}^{j}}\left(x_{i_{1}}, \ldots, x_{i_{j}}, c_{0}, c_{0}, c_{1}\right)$. Second, we implement every clause of the form $\left(\neg x_{i}\right)$ as $R_{\mathrm{IS}_{00}^{2}}\left(c_{1}, c_{1}, c_{1}, x_{i}, c_{1}\right)$. Third, we implement every clause of the form $\left(\neg x_{i_{1}} \vee x_{i_{2}}\right)$ as $R_{\mathrm{IS}_{00}^{2}}\left(x_{i_{2}}, c_{1}, x_{i_{1}}, c_{0}, c_{1}\right)$. Let $\varphi^{\prime}$ be the $W_{\mathrm{IS}_{00}}$-formula resulting from replacing every clause in $\varphi$ in the above manner. We see that $R\left(x_{1}, \ldots, x_{\kappa}\right) \equiv \exists c_{0} \exists c_{1} \cdot \varphi^{\prime}$, and since we in total only require 2 existentially quantified variables, it follows that $\mathrm{IS}_{00}$ is polynomially closed.

\subsection{Superpolynomially Closed Co-Clones}

From Section 4.3 and Section 4.4 we now have straightforward, sufficient conditions for verifying whether a given co-clone is polynomially closed. We now turn to the problem of determining whether a co-clone is not polynomially closed, i.e., superpolynomially closed. We show that this question is related to counting the number of $\kappa$-ary relations in a co-clone - a problem 
that has attracted significant attention in universal algebra and conceptual learning problems |BMMVW06 IMMVW10|. Before we can present this result we, for every finite domain $D$, introduce a particular constraint language $P_{D}$, which will turn out to be a plain base of $\operatorname{Rel}_{D}$. The language $P_{D}$ is defined as

$$
P_{D}=\left\{R \mid \kappa \geq 1, t \in D^{\kappa}, R=D^{\kappa} \backslash\{t\}\right\} .
$$

In other words each $\kappa$-ary relation in $P_{D}$ contains all $\kappa$-ary tuples over $D$ except one. Observe that $P_{\{0,1\}}$ is equivalent to the plain base of BR in Table 4.1

Lemma 4.11. For any finite domain $D$ the language $P_{D}$ is a plain base of $\operatorname{Re}_{D}$.

Proof. We must prove that $\left\langle P_{D}\right\rangle_{\nexists}=\operatorname{Rel}_{D}$, i.e. that we can q.f.p.p. define all relations over $D$. Hence, let $R \in \operatorname{Rel}_{D}$ be a $\kappa$-ary relation. For every $t \in D^{\kappa} \backslash R$ we let $R_{t} \in P_{D}$ denote the unique relation satisfying $R_{t}=D^{\kappa} \backslash\{t\}$. Hence, a constraint of the form $R_{t}\left(x_{1}, \ldots, x_{\kappa}\right)$ implies that $x_{1}, \ldots, x_{\kappa}$ can take any value except for $t[1], \ldots, t[\kappa]$. With this observation it is then easy to see that we can implement $R$ with the q.f.p.p. definition

$$
R\left(x_{1}, \ldots, x_{\kappa}\right) \equiv R_{t_{1}}\left(x_{1}, \ldots, x_{\kappa}\right) \wedge \ldots \wedge R_{t_{\mu}}\left(x_{1}, \ldots, x_{\kappa}\right),
$$

where $\left\{t_{1}, \ldots, t_{\mu}\right\}=D^{\kappa} \backslash R$. Note we in the above q.f.p.p. definition do not make use of explicit equality constraints.

Also observe that $\left\langle P_{D}^{(\leq \kappa)}\right\rangle_{\nexists \nsupseteq} \subseteq\left\langle P_{D}^{(\leq \kappa+1)}\right\rangle_{\nexists}$ for each $\kappa \geq 1$. We will now prove that if a co-clone $\operatorname{Inv}(\mathrm{C})$ contains a sufficiently large number of $\kappa$-ary relations, then for every polynomial $p$ there will exist some $\kappa$-ary relation in $\operatorname{Inv}(\mathrm{C})$ that $P_{D}$ cannot p.p. define using only $p(\kappa)$ existentially quantified variables. To make this counting argument more precise we, given a constraint language $S$, first let

$$
S^{(=\kappa)}=\{R \in S \mid \operatorname{ar}(R)=\kappa\}
$$

and then define the function $s_{S}$ as

$$
s_{S}(\kappa)=\log _{2}\left(\left|S^{(=\kappa)}\right|\right) .
$$

With this notation we see that $s_{\operatorname{Inv}(C)}(\kappa)$ denotes the exponent of the number of $\kappa$-ary relations in the co-clone $\operatorname{Inv}(\mathrm{C})$, and obtain the following lemma.

Lemma 4.12. Let $\operatorname{Inv}(\mathrm{C})$ be a co-clone of finite order over a finite domain $D$. If $\operatorname{Inv}(\mathrm{C})$ is polynomially closed, then $s_{\operatorname{Inv}(\mathrm{C})}(\kappa) \leq p(\kappa)$ for some polynomial $p$.

Proof. Let $S$ be a finite base of $\operatorname{Inv}(C)$ and let $R$ be the relation with the highest arity $\lambda$ in $S$. We make a few observations before the proof: first, $\langle S\rangle_{\nexists} \subseteq$ $\left\langle P_{D}^{(=\lambda)}\right\rangle_{\nexists}$; second, if some $R^{\prime} \notin\left\langle P_{D}^{(=\lambda)}\right\rangle_{\nexists}$ then $R^{\prime} \notin S$. This also implies that if $S$ can p.p. define some $\kappa$-ary relation $R$ with $p(\kappa)$ existentially quantified then the same is true for $P_{D}^{(=\lambda)}$. By contraposition this also implies that if $P_{D}^{(=\lambda)}$ cannot p.p. define some $\kappa$-ary relation $R$ with $p(\kappa)$ variables then neither can $S$. It is not too difficult to see that the number of q.f.p.p. implementations with $P_{D}^{(=\lambda)}$ over $\mathcal{\kappa}$ variables is bounded by $2^{|D|^{\lambda} \kappa^{\lambda}}$, since 
1. $P_{D}^{(=\lambda)}$ contains $|D|^{\lambda}$ relations, and

2. for each relation in $P_{D}^{(=\lambda)}$ one can form at most $\kappa^{\lambda}$ distinct constraints.

Since $\operatorname{Inv}(C)$ is polynomially closed, we are allowed to introduce at most $p(\kappa)$ existentially quantified variables to implement any $\kappa$-ary relation, hence, the number of definable relations is at most $2^{|D|^{\lambda} p(\kappa)^{\lambda}}$, which implies that $s_{\operatorname{Inv}(\mathrm{C})}(\kappa) \leq|D|^{\lambda} p(\kappa)^{\lambda}$.

Since the number of $\kappa$-ary relations over a finite domain $D$ is $2^{|D|^{\kappa}}$ it immediately follows that $\operatorname{Rel}_{D}$ is superpolynomially closed. To handle the other cases where it is not so obvious how to count the number of $\kappa$-ary relations we utilise a result from Berman et al. [BMMVW06]. Before we can present their result, we need a few additional preliminaries. If $S$ is a constraint language over $D$ the algebra $\mathbf{A}_{S}=(D, \operatorname{Pol}(S))$ is said to have few subpowers if $s_{\langle S\rangle}(\kappa) \in O\left(\kappa^{\lambda}\right)$ for some natural number $\lambda \geq 1$, and to have many subpowers if $c^{\mathcal{K}} \in O\left(s_{\langle S\rangle}(\mathcal{K})\right)$ for some real number $c>1$. An $n$-edge operation over $D$, $n \geq 2$, is an $(n+1)$-ary operation $f$ satisfying the $n$ identities

- $f(x, x, y, y, y, \ldots, y, y)=y$,

- $f(x, y, x, y, y, \ldots, y, y)=y$,

- $f(y, y, y, x, y, \ldots, y, y)=y$,

- $f(y, y, y, y, x, \ldots, y, y)=y$,

- $f(y, y, y, y, y, \ldots, x, y)=y$,

- $f(y, y, y, y, y, \ldots, y, x)=y$.

We then have the following useful theorem from Berman et al. |BMMVW06], restated in terms of our terminology of clones and co-clones

Theorem 4.13. [BMMVW06] Let $S$ be a constraint language over a finite domain $D$. If $\operatorname{Pol}(S)$ does not contain an $n$-edge operation for any $n \geq 2$ then (1) the algebra $(D, \operatorname{Pol}(S))$ has many subpowers and $(2) s_{\langle S\rangle}(\kappa) \notin O\left(\kappa^{m}\right)$ for any $m \geq 0$.

Hence, if $(D, \operatorname{Pol}(S))$ has many subpowers, then, intuitively, $\langle S\rangle$ contains too many relations for it to be polynomially closed. Combining Lemma 4.12 and Theorem 4.13 we obtain the following classification of the superpolynomially closed co-clones.

Theorem 4.14. Let $\mathrm{C}$ be a co-clone of finite order over a finite domain $D$. If $\mathrm{C}$ does not contain an n-edge operation for any $n \geq 2$ then $\operatorname{Inv}(C)$ is superpolynomially closed.

With the help of Table 2.1 one can verify that any Boolean co-clone of finite order above or equal to $\operatorname{Inv}(V), \operatorname{Inv}(E), \operatorname{or} \operatorname{Inv}(N)$ in Figure 4.3 , fullfil this property. 
Theorem 4.15. If $\operatorname{Inv}(\mathrm{C}) \supseteq \operatorname{Inv}(\mathrm{X})$ for some $\mathrm{X} \in\{\mathrm{V}, \mathrm{E}, \mathrm{N}\}$ then $\operatorname{Inv}(\mathrm{C})$ is superpolynomially closed.

Due to the close relationship between a polynomially closed co-clone and the existence of a polynomial, plain base, one might suspect that superpolynomially closed co-clones are unlikely to admit such polynomial bases. This can in fact be proven by a straightforward counting argument, using the bounds from Theorem 4.13 on the number of $\kappa$-ary relations in these coclones.

Theorem 4.16. Let $\operatorname{Inv}(\mathrm{C})$ be a superpolynomially closed co-clone over a finite domain $D$. If $S_{\mathrm{pl}}$ is a plain base of $\operatorname{Inv}(\mathrm{C})$ satisfying $\left|S_{\mathrm{pl}}^{(\leq \kappa)}\right| \leq 2^{p(\kappa)}$ for some polynomial $p$, then $S_{\mathrm{pl}}$ is not a polynomial base.

Proof. Assume that $S_{\mathrm{pl}}$ is a polynomial, plain base of $\operatorname{Inv}(\mathrm{C})$ with respect to a polynomial $c$. We show the theorem with a counting argument, using the results of Section 4.5 First, recall that $s_{\operatorname{Inv}(\mathrm{C})}(\kappa)$ cannot be bounded by a polynomial function since $\operatorname{Inv}(C)$ is superpolynomially closed. In other words it cannot hold that $|\{R \in \operatorname{Inv}(\mathrm{C}) \mid \operatorname{ar}(R)=\kappa\}| \leq 2^{g(\kappa)}$ for some polynomial $g$.

Now observe that for each $\kappa$-ary $R \in S_{\mathrm{pl}}$, there are at most $\kappa^{\mathcal{K}}$ different possible constraints one can form with $R$; thus the number of different possible constraints overall is bounded by $\left|S_{\mathrm{pl}}^{(\leq \kappa)}\right| \cdot \kappa^{\kappa}$. The number of possible formulas with at most $c(\kappa)$ constraints is then bounded by

$$
\left(\left|S_{\mathrm{pl}}^{(\leq \kappa)}\right| \cdot \kappa^{\kappa}\right)^{c(\kappa)} \leq\left(2^{p(\kappa)} \cdot \kappa^{\kappa}\right)^{c(\kappa)} \leq 2^{q(\kappa)}
$$

for a polynomial $q(\kappa)$, which implies that $s_{\operatorname{Inv}(C)}(\kappa) \leq q(\kappa)$, contradicting the assumption that $\operatorname{Inv}(C)$ is superpolynomially closed.

Using Table 4.1 we see that this property is fullfiled for all Boolean coclones. Hence, we obtain the following theorem.

Theorem 4.17. If $\operatorname{Inv}(\mathrm{C}) \supseteq \operatorname{Inv}(\mathrm{X})$ for some $\mathrm{X} \in\{\mathrm{V}, \mathrm{E}, \mathrm{N}\}$ then the plain base of $\operatorname{Inv}(\mathrm{C})$ in Table 4.1 is not a polynomial base.

Thus, a Boolean co-clone of finite order has a polynomial, plain base in Table 4.1 if and only if it is polynomially closed. In conjunction, the results of Section 4.3 and Section 4.5 therefore imply the following corollary.

Corollary 4.18. Let $\langle S\rangle$ be a Boolean co-clone of finite order. Then the following statements are equivalent.

- $\langle S\rangle$ is polynomially closed.

- $\langle S\rangle$ has a polynomial, plain base in Table 4.1

- The algebra $(\{0,1\}, \operatorname{Pol}(S))$ has few subpowers.

- There exists a polynomial $p$ such that the number of $\kappa$-ary relations in $\langle S\rangle$ is not larger than $2^{p(\kappa)}$. 
For arbitrary finite domains our result do not form a sharp dichotomy. Combining the results from Section 4.3 and Section 4.5 , we however obtain the following corollary.

Corollary 4.19. Let $\langle S\rangle$ be a co-clone of finite order over a finite domain. Then the following statements hold.

- If $\operatorname{Pol}(S)$ does not contain an n-edge operation for any $n \geq 2$ then $\langle S\rangle$ is superpolynomially closed.

- If $\operatorname{Pol}(S)$ contains an $n$-ary near-unanimity operation for some $n \geq 3$ then $\langle S\rangle$ is polynomially closed.

For co-clones of infinite order this situation differs drastically, as evident in Section 4.4. since even in the Boolean domain it can be the case that a co-clone of infinite order is polynomially closed even if the corresponding algebra has many subpowers.

\subsection{Strong Partial Clones of Finite and Infinite Order}

So far we have been interested in obtaining conditions for separating polynomially closed co-clones from superpolynomially closed co-clones, and obtained a complete dichotomy theorem for the Boolean domain. Since we for polynomially closed co-clones can implement all relations in the co-clone with a comparably few number of existentially quantified variables, one might conjecture that a strong partial clone $\operatorname{pPol}(S)$ has a more complex structure if $\langle S\rangle$ is superpolynomially closed. To make this intuition a bit more precise, given a co-clone $\operatorname{Inv}(\mathrm{C})$ and a base $S$ of $\operatorname{Inv}(\mathrm{C})$, we are interested in determining when $\mathrm{pPol}(S)$ is of infinite order and when it is of finite order. Hence, we make the following definition (recall from Definition 3.1 in Chapter 3 that $\operatorname{Int}_{[\cdot]_{s}}(\mathrm{C})$ denotes the interval of all strong partial clones where the total component equals $C$ ).

Definition 4.20. Let $\mathrm{C}$ be a clone over a finite domain. We say that $\operatorname{Int}_{[\cdot]_{s}}(\mathrm{C})$ is finitely generated if every $\operatorname{pPol}(S) \in \operatorname{Int}_{[\cdot]_{S}}(\mathrm{C})$ is of finite order and that $\operatorname{Int}_{[\cdot]_{S}}(\mathrm{C})$ is infinitely generated if $\mathrm{pPol}(S)$ is of infinite order for every $\operatorname{pPol}(\Delta) \in \operatorname{Int}_{[\cdot]_{s}}(\mathrm{C})$.

A few basic observations are in place. First, determining whether a partial clone is of finite or infinite order is a problem that has attracted significant attention in the literature, see e.g. [BH98: $\mathrm{CH} 10$; HF99]. However, observe that the authors in this case study partial clones that are not necessarily strong, and that a partial clone $\mathrm{P}$ might be of infinite order even though the smallest strong partial clone containing $P$ is of finite order. Second, if $C$ is a clone of finite order then $[C]_{S}$ is of finite order. Hence, in general, intervals of the form $\mathrm{Int}_{[\cdot]_{S}}(\mathrm{C})$ may contain strong partial clones of both finite and infinite order. If we restrict ourself to strong partial clones $\operatorname{pPol}(S)$ where $S$ is finite, this 
phenomenon is not as likely to occur, however. We thus make the following definition as well.

Definition 4.21. Let $C$ be a clone over a finite domain $D$. The finite interval of $C, \operatorname{Int}_{[\cdot]_{S}}^{\mathrm{fin}}(\mathrm{C})$, is the set $\operatorname{Int}_{[\cdot]_{S}}^{\mathrm{fin}}(\mathrm{C})=\left\{\operatorname{pPol}(S) \mid S \subseteq \operatorname{Rel}_{D}, S\right.$ is finite, $\mathrm{C}=$ $\operatorname{Pol}(S)\}$.

In Section 4.6.1 we prove that the existence of finitely generated intervals is related to the question of whether the cardinality of the interval is finite or uncountably infinite, and give examples of polynomially closed co-clones over arbitrary finite domains resulting in finitely generated intervals. Since not much is currently known about the lattice of strong partial clones over arbitrary finite domains, these results are necessarily inconclusive, and we cannot yet hope to provide a complete classification of finitely generated intervals. In Section 4.6.2 we study the opposite question: given a superpolynomially closed co-clone $\operatorname{Inv}(\mathrm{C})$, is $\operatorname{Int}_{[\cdot]_{s}}^{\mathrm{fin}}(\mathrm{C})$ infinitely generated? We give a general result and prove that $\operatorname{Int}_{[\cdot]_{S}}^{\mathrm{fin}}(\mathrm{C})$ is infinitely generated whenever $\mathrm{C}$ consists of essentially unary functions, i.e., if $\mathrm{C}=\left[\left\{e_{1}, \ldots, e_{m}\right\}\right]$ for some unary functions $e_{1}, \ldots, e_{m}$. The results are summarised in Figure 4.2

\subsubsection{Strong Partial Clones of Finite Order}

We first remark that if $\operatorname{Int}_{[]_{s}}(\mathrm{C})$ is finitely generated then the cardinality of $\operatorname{Int}_{[\cdot]_{s}}(\mathrm{C})$ is at most countably infinite. Hence, we get the following proposition.

Theorem 4.22. Let $\mathrm{C}$ be clone such that $\operatorname{Int}_{[\cdot]_{s}}(\mathrm{C})$ is of uncountably infinite cardinality. Then $\operatorname{Int}_{[\cdot]_{s}}(\mathrm{C})$ is not finitely generated.

On the other hand, if $\operatorname{Int}_{[\cdot]_{s}}(\mathrm{C})$ is finite and $\mathrm{C}$ is of finite order, it is not too difficult to see that $\operatorname{Int}_{\left[\cdot \cdot_{s}\right.}(C)$ must be finitely generated.

Lemma 4.23. Let $\mathrm{C}$ be a clone of finite order over D such that $\operatorname{Int}_{[\cdot]_{s}}(\mathrm{C})$ is finite. Then $\operatorname{Int}_{[\cdot]_{s}}(\mathrm{C})$ is finitely generated.

Proof. Let $F$ denote an arbitrary finite base of $C$. Then $[F]_{s}$ is the least element in $\operatorname{Int}_{[\cdot]_{s}}(C)$. Assume there exists a strong partial clone $C^{\prime} \in \operatorname{Int}_{[\cdot]_{s}}(C)$ of infinite order. Obviously $C^{\prime} \supset[F]_{s}$. Let $f \in C^{\prime} \backslash[F]_{s}$. Then it is easy to see that $C^{\prime} \supset[F \cup\{f\}]_{s} \supset[F]_{s}$ since by assumption $C^{\prime}$ is of infinite order and cannot be generated by $F \cup\{f\}$. This procedure can be repeated arbitrarily many times, which contradicts the assumption that $\operatorname{Int}_{[\cdot]_{s}}(\mathrm{C})$ was finite.

Hence, whether an interval is finitely generated or not is tightly connected to whether the interval is finite. In the Boolean domain it has been proven that $\operatorname{Int}_{[\cdot]_{s}}(\operatorname{Pol}(S))$ is finite if $\langle S\rangle$ is a subset of $\mathrm{IM}_{2}$ or $\mathrm{ID}_{1}$, and is of uncountably infinite cardinality otherwise [Sch15]. Hence, we obtain the following proposition. 


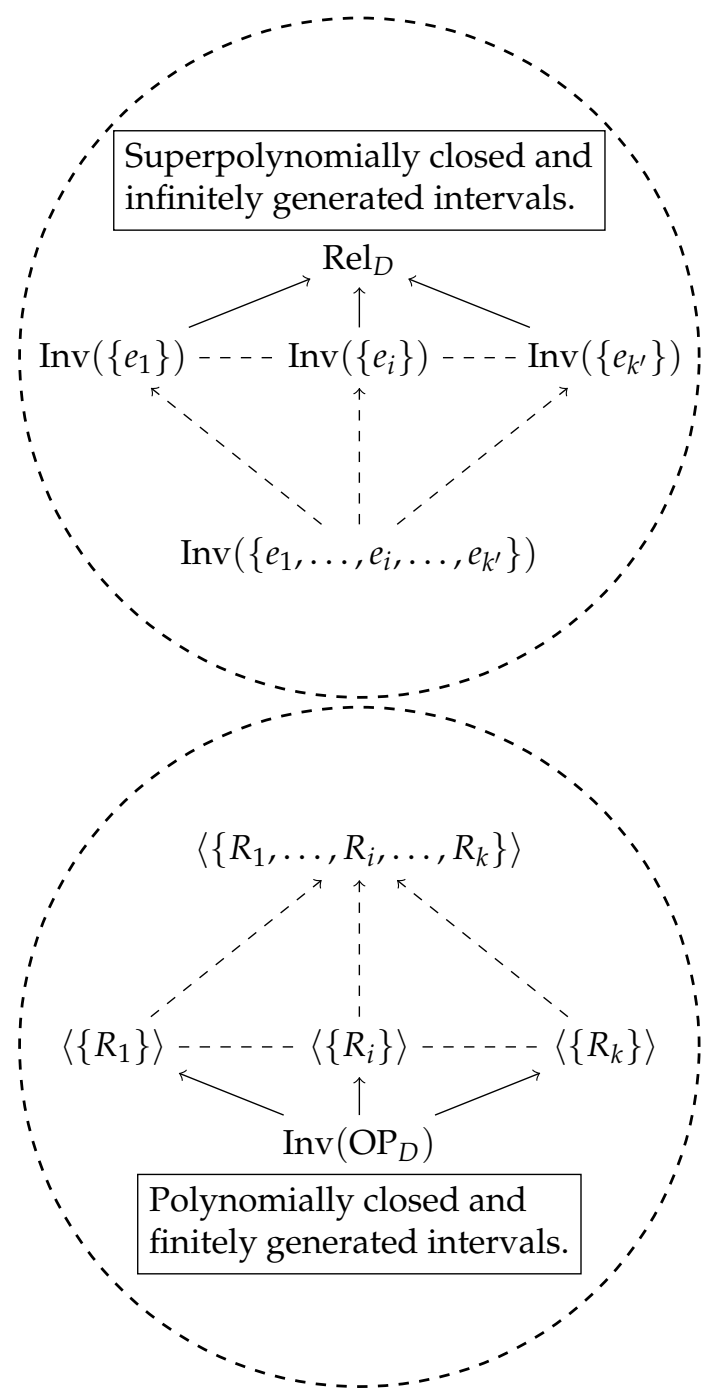

Figure 4.2: An illustration of the structure of $\operatorname{Int}_{[\cdot]_{S}}(S)$ and $\operatorname{Int}_{[\cdot]_{S}}^{\mathrm{fin}}(S)$ for $S$ over an arbitrary finite domain $D=\{1, \ldots, i, \ldots, k\}$. For $a \in D$ let $R_{a}$ denote the relation $\{(a)\}$. Let $e_{1}, \ldots, e_{k^{\prime}}, k^{\prime}=k^{k}-1$, be an enumeration of the unary functions over $D$ distinct from $\pi_{1}^{1}$. A directed arrow from node $A$ to $B$ means that $A \subset B$. A dashed arrow from node $A$ to $B$ means that there exists $A^{\prime}$ such that $A \subset A^{\prime} \subset B$. Some inclusions have been omitted. 
Proposition 4.24. Let $S$ be a Boolean constraint language. Then $\operatorname{Int}_{[\cdot]_{S}}(\operatorname{Pol}(S))$ is finitely generated if and only if $\langle S\rangle \subseteq \operatorname{Inv}(\mathrm{X})$ for $\mathrm{X} \in\left\{\mathrm{M}_{2}, \mathrm{D}_{1}\right\}$.

In Schölzel [Sch15] it is conjectured that intervals of the form $\operatorname{Int}_{[\cdot]_{s}}(\operatorname{Pol}(S))$ are either finite or uncountably infinite for arbitrary finite domains. Such a dichotomy theorem would therefore also answer the question which intervals are finitely generated and which are not. We remark that such a dichotomy theorem is likely very difficult to obtain, since not much is known of the structure of the lattice of strong partial clones over arbitrary finite domains. We give an example of a simple kind of constraint language where the intervals of strong partial clones is always finite.

Given a finite domain $D=\{0, \ldots, k\}$ let $R_{i}, i \in D$, denote the unary, constant relation $\{(i)\}$. Say that a co-clone $\operatorname{Inv}(C)$ over $D$ is essentially constant if there exists a set $S \subseteq\left\{R_{0}, \ldots, R_{k}\right\}$ such that $\langle S\rangle=\operatorname{Inv}(\mathrm{C})$. In other words $\operatorname{Inv}(C)$ can be generated from a finite set of constant relations.

Theorem 4.25. Let $\operatorname{Inv}(\mathrm{C})$ be an essentially constant co-clone over some finite domain D. Then $\operatorname{Int}_{[\cdot]_{s}}(\mathrm{C})$ is finitely generated.

Proof. Since $\operatorname{Inv}(\mathrm{C})$ is essentially constant there exists $S \subseteq\left\{R_{0}, \ldots, R_{k}\right\}$ such that $\langle S\rangle=\operatorname{Inv}(\mathrm{C})$. It is known both that $\operatorname{Pol}\left(\left\{R_{0}, \ldots, R_{k}\right\}\right)$, the clone consisting of all idempotent functions over $D$, is finitely generated Qua71| and that there exists a finite number of (strong) partial clones containing $\operatorname{Pol}\left(\left\{R_{0}, \ldots, R_{k}\right\}\right)[\overline{F H 98}]$. From this it easily follows that $\operatorname{Pol}(S)$ is of finite order and that $\operatorname{Int}_{[\cdot]_{s}}(\mathrm{C})$ is finite. By applying Lemma 4.23 it follows that $\operatorname{Int}_{[\cdot]_{s}}(\mathrm{C})$ is finitely generated.

The reader might well ask why we do not attempt to prove a more general result than Theorem 4.25. The reason is that, currently, not much is known about the structure of finitely generated intervals of strong partial clones over arbitrary finite domains. For instance, it is not even known whether $\operatorname{pPol}\left(\left\{u_{1}, \ldots, u_{n}\right\}\right)$, where each $u_{i} \subseteq D$, is of finite order. Moreover, it is known that the intersection of two strong partial clones of finite order can be of infinite order [CH10], which suggests that this problem is more difficult than one might believe at a first glance.

\subsubsection{Strong Partial Clones of Infinite Order}

We now turn to the problem of determining whether an interval $\operatorname{Int}_{[\cdot]_{s}}^{\mathrm{fin}}(\mathrm{C})$ is infinitely generated. We show that $\operatorname{Int}_{[\cdot]_{s}}^{\mathrm{fin}}(\mathrm{C})$ is always infinitely generated if $C$ is an essentially unary clone over an arbitrary finite domain $D$. For finite Boolean constraint languages $S$ this implies that if $\langle S\rangle \supseteq \operatorname{IN}_{2}$, i.e. $\operatorname{CSP}(S)$ is $\mathrm{NP}$-complete assuming $\mathrm{P} \neq \mathrm{NP}$, then $\operatorname{pPol}(S)$ is of infinite order. For the proofs, we first need the following construction of a universal hash family, due to Alon et al. AYZ95]. 
Theorem 4.26 (Section 4 of |AYZ95]). For any $k$ and $n$, there is a family $H$ of $2^{O(k)} \log (n)$ functions $h_{i}:\{1, \ldots, n\} \mapsto\{1, \ldots, k\}$ such that for every $S \subset\{1, \ldots, n\}$ of size $k$ there is a function in $H$ that is injective on $S$.

Note that the bound $O(k)$ has no hidden dependency on $n$. Hence, if $k$ is a constant, then $2^{O(k)} \log (n) \in O(\log (n))$. The purpose of a universal hash family in this section is to, given a $\kappa$-ary relation $R$, create a $\kappa^{\prime}$-ary relation $R^{\prime}$ using the universal hash family such that $\operatorname{pPol}(\{R\}) \subseteq \operatorname{pPol}\left(\left\{R^{\prime}\right\}\right)$, and such that $\kappa^{\prime}=O(\kappa)$. In the following definition we exploit the fact that any $\kappa$-ary relation $R$ can be viewed as an $|R| \times \kappa$ matrix where each row corresponds to a tuple in $R$.

Definition 4.27. Let $R$ be a relation over $D,|R|=\mu$, let $r \geq 1$ and let $H$ be the universal hash family from $\{1, \ldots, \mu\}$ to $\{1, \ldots, r\}$. The closure of $R$ under $H, H(R)$, is the relation defined as follows.

1. let $M$ be the matrix corresponding to $R$,

2. let $g_{1}, \ldots, g_{|D|^{r}}$ be an enumeration of all functions $g:\{1, \ldots, r\} \mapsto D$,

3. for every $h_{i} \in H$ and every $g_{j}$ add the column $y_{i, j}$ to $M$ which in row $x \in\{1, \ldots, \mu\}$ takes the value $g_{j}\left(h_{i}(x)\right)$,

4. let $H(R)$ be the relation corresponding to $M$.

Say that a relation $R$ over $D$ is $n$-saturated if for every $t_{1}, \ldots, t_{n^{\prime}} \in R, n^{\prime} \leq$ $n$, for every $\left(x_{1}, \ldots, x_{n^{\prime}}\right) \in D^{n^{\prime}}$ there exists an $i$ such that $\left(t_{1}[i], \ldots, t_{n^{\prime}}[i]\right)=$ $\left(x_{1}, \ldots, x_{n^{\prime}}\right)$.

Lemma 4.28. Let $R$ be a relation with $\mu$ tuples and let $r \geq 1$. Let $H$ be the universal hash family from $\{1, \ldots, \mu\}$ to $\{1, \ldots, r\}$. Then $H(R)$ is $r$-saturated.

Proof. Let $t_{1}, \ldots, t_{q} \in H(R), q \leq r$, and let $M$ be the matrix corresponding to $H(R)$. For every $\left(x_{1}, \ldots, x_{q}\right) \in D^{q}$ we must prove that there exists some $j$ such that $\left(x_{1}, \ldots, x_{q}\right)=\left(t_{1}[j], \ldots, t_{q}[j]\right)$. Let $P=\left(p_{1}, \ldots, p_{q}\right) \in\{1, \ldots, \mu\}^{q}$ be the row indices of $t_{1}, \ldots, t_{q}$, i.e., $t_{i}=M\left[p_{i}, \cdot\right]$ for each $i \in\{1, \ldots, q\}$. Since $H$ is a universal hash family, there is some $h \in H$ which is injective on $P$. Let $g: D^{q} \mapsto D$ be the function satisfying $\left(g\left(h\left(p_{1}\right)\right), \ldots g\left(h\left(p_{q}\right)\right)\right)=\left(x_{1}, \ldots, x_{q}\right)$. Due to the construction of $H(R)$ this implies that the column in $M$ corresponding to $h$ and $g$ will enumerate $\left(x_{1}, \ldots, x_{q}\right)$. Hence, there is a $j$ such that $\left(x_{1}, \ldots, x_{q}\right)=\left(t_{1}[j], \ldots, t_{q}[j]\right)$.

Recall from Section 3.2 in Chapter 3 that if $R$ is a relation and $S$ a constraint language then $\operatorname{Pol}(S)(R)$ denotes the smallest superset of $R$ which is closed under $\operatorname{Pol}(S)$.

Lemma 4.29. Let $\operatorname{Pol}(S)$ be an essentially unary clone. If $\operatorname{pPol}(S)$ is of finite order, then $S$ can p.p. implement any $\kappa$-ary relations $R \in\langle S\rangle$ with at most $O(\kappa)$ existentially quantified variables. 
Proof. Let $R$ be a $\kappa$-ary relation in $\langle S\rangle$, and let $\mu \leq|D|^{\kappa}$ be the number of tuples in $R$. Let $F$ be a finite base of $\operatorname{pPol}(S)$, let $r$ be the largest arity of any function in $F$, and let $H$ be the $r$-universal hash family from $\{1, \ldots, \mu\}$ to $\{1, \ldots, r\}$ of Theorem 4.26 . Let $R^{\prime}=H(R)$. By the construction of $H(R)$ in Definition 4.27 it follows that $\operatorname{ar}\left(R^{\prime}\right)=\kappa+|D|^{r}|H|=\kappa+|D|^{r} 2^{O(r)} \log (\mu)=$ $|D|^{r} 2^{O(r)} O(\kappa)$. To see that the last equality holds simply note that $\log (\mu) \leq$ $\log \left(|D|^{\kappa}\right)=O(\kappa)$. Moreover, since $r$ is a constant, it also holds that $\operatorname{ar}\left(R^{\prime}\right)=$ $O(\kappa)$. Let $\kappa^{\prime}=\operatorname{ar}\left(R^{\prime}\right)$, and let $R^{\prime \prime}=\operatorname{Pol}(S)\left(R^{\prime}\right)$, i.e. $R^{\prime}$ closed under all polymorphisms of $S$. Obviously, $\operatorname{Pol}(S) \subseteq \operatorname{Pol}\left(\left\{R^{\prime \prime}\right\}\right)$. Next, note that

$$
R\left(x_{1}, \ldots, x_{\kappa}\right) \equiv \exists x_{\kappa+1}, \ldots, x_{\kappa^{\prime}} R^{\prime \prime}\left(x_{1}, \ldots, x_{\kappa}, x_{\kappa+1}, \ldots, x_{\kappa^{\prime}}\right),
$$

or, put in other words, $R^{\prime \prime}$ can p.p. define $R$ with at most $O(\kappa)$ existentially quantified variables. To see that this holds, simply note that $\left\{\left(x_{1}, \ldots, x_{\kappa}\right) \mid\right.$ $\left.\left(x_{1}, \ldots, x_{\kappa}, x_{\kappa+1}, \ldots, x_{\kappa^{\prime}}\right) \in R^{\prime \prime}\right\}=R$.

It remains to prove that $\operatorname{pPol}(S) \subseteq \operatorname{pPol}\left(\left\{R^{\prime \prime}\right\}\right)$, since this, due to the Galois connection, implies that $\left\langle\left\{R^{\prime \prime}\right\}\right\rangle_{\exists} \subseteq\langle S\rangle_{\nexists \exists}$ and that $S$ can p.p. define $R$ using at most $O(\kappa)$ existentially quantified variables. Hence, let $f \in F$ be a $q$-ary, $q \leq r$, function. If $f \notin \operatorname{pPol}\left(\left\{R^{\prime \prime}\right\}\right)$ then there exists $t_{1}, \ldots, t_{q} \in R^{\prime \prime}$ such that $f\left(t_{1}, \ldots, t_{q}\right) \notin R^{\prime \prime}$. We may assume that all $t_{1}, \ldots, t_{q}$ are distinct, as otherwise the application of $f$ is equivalent to the application of some $q^{\prime}$-ary partial polymorphism $f^{\prime}$ on distinct rows, where $q^{\prime}$ is the number of distinct rows represented in $\left(t_{1}, \ldots, t_{q}\right){ }^{1}$

Our strategy is now, using Lemma 4.28 , to prove that we can define a total function $h$ using the partial function $f$ such that $h$ does not preserve $R^{\prime \prime}$. However, this also implies that $h \notin \operatorname{Pol}(S)$, which is a contradiction since $f \in$ $\operatorname{pPol}(S)$. Before this proof we make one observation: for every $t \in R^{\prime \prime}$ there exists $t^{\prime} \in R^{\prime}$ and a unary function $u \in \operatorname{Pol}(S)$ such that $u\left(t^{\prime}\right)=t$. Hence, for the tuples $t_{1}, \ldots, t_{q}$ there exists $t_{1}^{\prime}, \ldots, t_{q}^{\prime} \in R^{\prime}$ and $u_{1}, \ldots, u_{q} \in \operatorname{Pol}(S)$ such that $u_{i}\left(t_{i}^{\prime}\right)=t_{i}$. We now define the $q$-ary function $h$ as

$$
h\left(x_{1}, \ldots, x_{q}\right)=f\left(u_{1}\left(\pi_{1}^{q}\left(x_{1}, \ldots, x_{q}\right)\right), \ldots, u_{q}\left(\pi_{q}^{q}\left(x_{1}, \ldots, x_{q}\right)\right)\right) .
$$

Obviously, $h \in \operatorname{pPol}(S)$ since it is a composition of $f, u_{1}, \ldots, u_{q}$, and projection functions. This in turn implies that

$$
h\left(t_{1}^{\prime}, \ldots, t_{q}^{\prime}\right)=f\left(u_{1}\left(t_{1}^{\prime}\right), \ldots, u_{q}\left(t_{q}^{\prime}\right)\right)=f\left(t_{1}, \ldots, t_{q}\right) \notin R^{\prime \prime},
$$

but since $t_{1}^{\prime}, \ldots, t_{q}^{\prime} \in R^{\prime}, R^{\prime}$ is $r$-saturated and $q \leq r, h$ must be a total polymorphism, i.e. $h \in \operatorname{Pol}(S) \subseteq \operatorname{Pol}\left(\left\{R^{\prime \prime}\right\}\right)$. This is a contradiction since $h\left(t_{1}^{\prime}, \ldots, t_{q}^{\prime}\right) \notin R^{\prime \prime}$. Hence, $f \in \operatorname{pPol}\left(\left\{R^{\prime \prime}\right\}\right)$.

With the help of this Lemma we can now prove that $\mathrm{pPol}(S)$ is of infinite order whenever $S$ is finite and $\operatorname{Pol}(S)$ is essentially unary.

Theorem 4.30. Let $\mathrm{C}$ be an essentially unary clone over a finite domain $D$. Then $\operatorname{Int}_{[\cdot]_{s}}^{\mathrm{fin}}(\mathrm{C})$ is infinitely generated.

\footnotetext{
${ }^{1}$ The reader should note that this fact is one of the key observations that will be used in the forthcoming chapter.
} 
Proof. Let $S$ be a finite constraint language such that $\operatorname{Pol}(S)=\mathrm{C}$. Assume that $\operatorname{pPol}(S)$ can be finitely generated. By Lemma 4.29 we then have that $S$ can p.p. implement any $\kappa$-ary relations in $\operatorname{Inv}(\mathrm{C})$ with $O(\kappa)$ existentially quantified variables. However, this is a contradiction since $\langle S\rangle$ is superpolynomially closed by Theorem 4.14 . To see this simply note that $C$ cannot contain an $n$-edge operation for any $n \geq 2$ since an $n$-edge operation by definition is not essentially unary. This fact together with Lemma 4.12 results in a contradiction. Hence, $\mathrm{pPol}(S)$ cannot be of finite order.

This theorem has a number of interesting applications. First, recall that for Boolean constraint languages $S, \operatorname{SAT}(S)$ is NP-complete if and only if $\operatorname{Pol}(S) \subseteq[\neg x]$. Hence, assuming $\mathrm{P} \neq \mathrm{NP}, \operatorname{pPol}(S)$ is of infinite order whenever $S$ is finite and SAT $(S)$ is NP-complete. This implies that describing partial polymorphisms of finite constraint languages resulting in NP-complete CSP problems is a very difficult problem. For some illustrative usages of this theorem, let $R_{1 / \kappa}, \Gamma_{\mathrm{SAT}}^{k}$ and $\Gamma_{\mathrm{NAE}}^{k}$ be defined as in Section 2.3.2 in Chapter 2 , and recall that for $\kappa \geq 3, \operatorname{SAT}\left(\left\{R_{1 / \kappa}\right\}\right), \operatorname{SAT}\left(\Gamma_{\mathrm{NAE}}^{\kappa}\right)$, and $\operatorname{SAT}\left(\Gamma_{\mathrm{SAT}}^{\kappa}\right)$ can be seen as alternative formulations of the well-known NP-complete problems MONOTONE 1-IN- $\kappa-S A T$, NOT-ALL-EQUAL- $\kappa-S A T$, and $\kappa-S A T$, respectively. Since all these languages are finite we obtain the following corollary to Theorem 4.30 .

Corollary 4.31. Let $\kappa \geq 3$. Then $\mathrm{pPol}\left(\Gamma_{\mathrm{SAT}}^{\kappa}\right), \operatorname{pPol}\left(\Gamma_{\mathrm{NAE}}^{\kappa}\right)$, and $\mathrm{pPol}\left(\left\{R_{1 / \kappa}\right\}\right)$ are of infinite order.

It is worth noting that a complete dichotomy theorem for $\operatorname{CSP}(S)$ for constraint languages $S$ defined over arbitrary finite domains is not yet known. However, if $\operatorname{Pol}(S)$ is essentially unary and every $f \in \operatorname{Pol}(S)$ is injective, then $\operatorname{CSP}(S)$ is NP-complete [JCG97]. Hence, Theorem 4.30 also extends to many non-Boolean cases where $\operatorname{CSP}(S)$ is NP-complete.

\subsection{Summary and Conclusions}

We have studied the question of whether a polynomial amount of variables is sufficient to implement any relation in a given co-clone, have provided a complete dichotomy theorem for the Boolean co-clones where this is possible, and have also given several general results for arbitrary finite domains. Using these notions we have then studied the question of whether a given strong partial clone is of finite or infinite order, and proven that the latter holds for a large variety of well-studied constraint languages. In particular, the strong partial clones of interest in this thesis are almost always of infinite order. However, as we expand upon in the next section, it is possible to describe these strong partial clones by finite sets of partial functions. 



\section{Bounded Bases of Strong Partial Clones}

- Bring you my coat?

- Yes, sir, there is it.

- Button me. It pinches me too much upon stomack.

English as she is spoke

In this chapter we consider the implications of Theorem 4.30 and propose a method to describe the partial polymorphisms of any finite constraint language $S$ with a finite set of partial functions.

\subsection{Introduction}

The results in Chapter 4 have probably left a rather bleak picture of the applicability of partial clone theory to study the computational complexity of CSP and SAT problems. For the Boolean domain, not only are the two intervals $\operatorname{Int}_{\cdot ._{s}}\left(\operatorname{Pol}\left(\left\{R_{1 / 3}\right\}\right)\right)$ and $\operatorname{Int}_{\cdot \cdot]_{s}}\left(\operatorname{Pol}\left(\left\{R_{\mathrm{NAE}}\right\}\right)\right)$ of uncountably infinite order [Sch15], but according to Theorem 4.30 in Chapter 4 , whenever $\mathrm{pPol}(S)$ is included in any of these two intervals and $S$ is finite, then $\operatorname{pPol}(S)$ is of infinite order. Hence, assuming $\mathrm{P} \neq \mathrm{NP}$, for all finite Boolean constraint languages $S$ such that $\mathrm{SAT}(S)$ is NP-complete $\mathrm{pPol}(S)$ is of infinite order. For example, there does not exist any finite set of partial functions $F$ such that $[F]_{s}=\operatorname{pPol}\left(\left\{R_{1 / 3}\right\}\right)$.

In this chapter we consider methods to describe strong partial clones of infinite order, by introducing a new closure operator for partial functions, 
$\mathrm{Cl}(\cdot)$, and prove that there for any finite constraint language $S$ exists a finite set $F \subseteq \operatorname{pPol}(S)$ such that $C l(F)=\operatorname{pPol}(S)$. Hence, in general, the operator $\mathrm{Cl}(\cdot)$ is stronger than $[\cdot]_{s}$. We begin in Section 5.3 by introducing the notions of contraction and stretching of a partial function. If a partial function is contractable then, informally, this means that two or more arguments are identical whenever the function is defined. The function obtained by identifying these arguments is called the contraction of the function while the function obtained by duplicating one or more of its argument is called a stretching. With these notions we then define the bounded base of a strong partial clone. Given a finite constraint language $S$ the bounded basis is defined to be a finite subset of $\operatorname{pPol}(S)$, where the bound on the included functions depends only of the size of $S$, i.e. the cardinality and arity of the included relations. Let $B(S)$ denote this set of partial functions for some finite constraint language $S$, and observe that in general $[B(S)]_{S} \subset \operatorname{pPol}(S)$. Hence, we need a way to obtain the functions included in the set $\mathrm{pPol}(S) \backslash[B(S)]_{S}$.

To accomplish this, we in Section 5.4 first define the union of a set of partial functions $G$ as the function $\mathrm{u}(G)$ defined by $\mathrm{u}(G)\left(x_{1}, \ldots, x_{n}\right)=$ $g\left(x_{1}, \ldots, x_{n}\right)$ if there exists $g \in G$ such that $g$ is defined, and undefined for this sequence of arguments otherwise. It is not too hard to see that if no restrictons are induced on the set $G$ then this operator is too strong to be useful. We therefore introduce two notions to restrict this operator. The first one, consistency, says that any functions in $G$ returns the same value, or are undefined, when applied to the same sequence of arguments. The second one, $k$-covering, means that whenever we take some functions from $G$ which together are not defined on more than $k$ sequences of arguments, then $G$ also contains the union of these functions. Together these two restrictions guarantee the safety of the union operator. We let $\mathrm{Cl}(B(S))$ denote the set of all functions obtained by the union operator to stretchings of consistent and $k$ covering subsets of $B(S)$, and then prove that $C l(B(S))=\operatorname{pPol}(S)$. In other words, any function in $\mathrm{pPol}(S)$ can be defined in terms of the union of a set of stretchings of functions from the bounded basis. Hence, the bounded basis is expressive enough to characterise the strong partial clone of any finite constraint language.

Finally, we turn to the problem of determining bounded bases of finite Boolean constraint languages (Section 5.5. We first give some general results regarding Boolean constraint languages that contain additional sign patterns, i.e., relations representing negated arguments, and prove that in many cases the partial polymorphisms of such languages can be described in terms of simpler relations. Then we provide descriptions of the bounded bases for the two languages $\left\{R_{1 / \kappa}\right\}$ and $\Gamma_{\mathrm{SAT}}^{\kappa}$, which, thanks to the notions introduced in this chapter, have a particularly simple form. 


\subsection{Preliminaries}

For a finite set of partial functions $F$ over a finite domain we let $\operatorname{ar}(F)=$ $\max _{f \in F}(\operatorname{ar}(f))$. Similarly, if $S$ is a finite constraint language over a finite domain we let $\operatorname{ar}(S)=\max _{R \in S}(\operatorname{ar}(R))$. Given a relation $R \subseteq D^{\mathcal{K}}$ over a finite domain we let \#tuples $(R)=|R|$, i.e., the number of tuples in the relation, and if $S$ is a finite constraint language over a finite domain $D$ we let \#tuples $(\Gamma)=$ $\max _{R \in \Gamma}(\#$ tuples $(R))$. Note that \#tuples $(S) \leq|D|^{\operatorname{ar}(S)}$ always holds.

Recall from Section 2.1 in Chapter 2 that for $\kappa \geq 0$ and $c \in\{0,1\}$, we write $\vec{c}^{\kappa}$ for a $\kappa$-ary sequence of $0^{\prime}$ s or $1^{\prime}$ s. If $\kappa$ is clear from the context we simply write $\vec{c}$. If $R$ is a $\kappa$-ary relation and $t_{1}, \ldots, t_{n} \in$ $R$ a sequence of $n$ tuples then we let $\operatorname{Cols}\left(t_{1}, \ldots, t_{n}\right)$ be the sequence $\left(\left(t_{1}[1], \ldots, t_{n}[1]\right), \ldots,\left(t_{1}[\kappa], \ldots, t_{n}[\kappa]\right)\right)$. In other words, $\operatorname{Cols}\left(t_{1}, \ldots, t_{n}\right)$ are the columns in the $n \times \kappa$ matrix formed by letting each element $a_{i, j}=t_{i}[j]$. For instance, $\operatorname{Cols}((0,1,1),(1,1,1))$ is the sequence of tuples $((0,1),(1,1),(1,1))$. Whenever convenient we also use $\operatorname{ColsSet}\left(t_{1}, \ldots, t_{\kappa}\right)$ for the set (instead of a sequence) $\left\{\left(t_{1}[1], \ldots, t_{n}[1]\right), \ldots,\left(t_{1}[\kappa], \ldots, t_{n}[\kappa]\right)\right\}$.

\subsection{Bounded Bases}

In this section we give our first contribution of this chapter, namely, that for any finite constraint language $S$, it is possible to determine a finite set of partial functions which completely describes $\mathrm{pPol}(S)$. This finite set of partial functions is referred to as a bounded base. We start with an investigation of how functions with small arities characterise the partial polymorphisms of $S$. Intuitively, if $f$ has 'high' arity, we will consider sets of variables which have the same value in all the tuples over which $f$ is defined, and consider the contraction of $f$ obtained by removing such duplicates.

Definition 5.1 (redundant variable). Let $g$ be an $n$-ary partial function over arguments $x_{1}, \ldots, x_{n}$. A variable $x_{i}$ is said to be redundant with $x_{j}$ for $g(j \neq i)$, if $u[i]=u[j]$ for all $u \in \operatorname{domain}(g)$.

If $f$ is an $n$-ary function and $\sigma$ is a permutation on $[n]$, then we denote by $g \circ \sigma$ the function defined by $g \circ \sigma\left(x_{1}, \ldots, x_{n}\right)=g\left(x_{\sigma(1)}, \ldots, x_{\sigma(n)}\right)$ for all $x_{1}, \ldots, x_{n}$.

Definition 5.2 (contraction, stretching). Let $g$ be an $n$-ary partial function over arguments $x_{1}, \ldots, x_{n}$. If there is a permutation $\sigma$ of $[n]$ and a function $\gamma$ from $\{m+1, \ldots, n\}$ to $[m]$ such that for all $i \in\{m+1, \ldots, n\}$, $x_{i}$ is redundant with $x_{\gamma(i)}$ for $g \circ \sigma$, then the m-contraction of $g$ with respect to $\sigma$ and $\gamma$ is the $m$-ary function $g^{\prime}$ defined by $g^{\prime}\left(x_{1}, \ldots, x_{m}\right)=g \circ$ $\sigma\left(x_{1}, \ldots, x_{m}, x_{\gamma(m+1)}, \ldots, x_{\gamma(n)}\right)$. In this case $g$ is also said to be an $n$-stretching of $g^{\prime}$ with respect to $\sigma$ and $\gamma$.

Example 5.3. Let $g$ be the 6-ary partial function defined by $g(0,0,0,0,1,1)=$ $g(0,1,0,0,0,0)=0, g(1,1,1,1,0,0)=1$, and undefined otherwise. With 
the permutation $\sigma=(1,2,3,5,4,6)$ we get $g \circ \sigma(0,0,0,1,0,1)=0, g \circ$ $\sigma(0,1,0,0,0,0)=0$, and $g \circ \sigma(1,1,1,0,1,0)=1$. Now with $\gamma(5)=1$ and $\gamma(6)=4$ we can see that for $g \circ \sigma, x_{5}$ is redundant with $x_{\gamma(5)}$ and $x_{6}$ is redundant with $x_{\gamma(6)}$. Then the 4-contraction of $g$ with respect to $\sigma$ and $\gamma$ is the 4 -ary function $g^{\prime}$ defined by $g^{\prime}\left(x_{1}, x_{2}, x_{3}, x_{4}\right)=g \circ \sigma\left(x_{1}, x_{2}, x_{3}, x_{4}, x_{1}, x_{4}\right)$, that is, by $g^{\prime}(0,0,0,1)=g \circ \sigma(0,0,0,1,0,1)=g^{\prime}(0,1,0,0)=0, g^{\prime}(1,1,1,0)=1$, and undefined otherwise. Observe that a contraction is not necessarily maximal, in the sense that the $m$-contraction of a function may itself have an $\mathrm{m}^{\prime}$ contraction for some $m^{\prime}<m$, as is the case in this example ( $x_{3}$ is redundant with $x_{1}$ for $g^{\prime}$ ).

If $g^{\prime}$ is the $m$-contraction of $g$ with respect to some permutation $\sigma$ and function $\gamma$, we simply say that $g^{\prime}$ is an $m$-contraction of $g$ (and that $g$ is an $n$-stretching of $g^{\prime}$ ). If an $n$-ary function $f$ has no $m$-contractions for some $m<n$, then we say that $f$ is uncontractable to arity $m$. For instance, if $f$ is an $n$-ary function defined only on tuples of Hamming weight 1 , then it is uncontractable to arity $n-1$ since for all variables $x_{i} \neq x_{j}$, there is a tuple in the domain of $f$ which assigns 1 to $x_{i}$ and 0 to $x_{j}$ and hence, $x_{i}$ is not redundant with $x_{j}$ for $f$. Note that if $f$ is uncontractable to arity $m$, then it is a fortiori uncontractable to any arity $m^{\prime} \leq m$.

Given a set of partial functions $F$, we write $\mathcal{S}_{n}(F)$ for the set of all $n$-ary partial functions that are an $n$-stretching of some $f \in F$, and $\mathcal{C}_{m}(F)$ for the set of all $m$-ary partial functions that are an $m$-contraction of some $f \in F$. For $m \leq n$ we let $\mathcal{U}_{n}^{m}$ be the set of all $n$-ary partial functions uncontractable to arity $m$. The following is a simple but essential property.

Lemma 5.4. Let $g^{\prime}$ be an m-contraction of an $n$-ary partial function $g$. Then $\left[\left\{g^{\prime}\right\}\right]_{s}=[\{g\}]_{s}$ holds.

Proof. Since $g^{\prime}$ is an $m$-contraction of $g$ there exists $\sigma$ and $\gamma$ for which $g^{\prime}\left(x_{1}, \ldots, x_{m}\right)=g \circ \sigma\left(x_{1}, \ldots, x_{m}, x_{\gamma(m+1)}, \ldots, x_{\gamma(n)}\right)$. Recall that $\pi_{i}^{n}$ denotes the $i$ th projection function of arity $n$, and write $\vec{x}$ for the tuple $\left(x_{1}, \ldots, x_{m}\right)$. Then we have

$$
g^{\prime}(\vec{x})=g \circ \sigma\left(\pi_{1}^{m}(\vec{x}), \ldots, \pi_{m}^{m}(\vec{x}), \pi_{\gamma(m+1)}^{m}(\vec{x}), \ldots, \pi_{\gamma(n)}^{m}(\vec{x})\right)
$$

and

$$
g \circ \sigma\left(x_{1}, \ldots, x_{n}\right)=g\left(\pi_{\sigma(1)}^{n}\left(x_{1}, \ldots, x_{n}\right), \ldots, \pi_{\sigma(n)}^{n}\left(x_{1}, \ldots, x_{n}\right)\right) .
$$

Hence, $g^{\prime}$ can be obtained by composition of $g$ and projection functions, from which it follows that $g^{\prime} \in[\{g\}]_{s}$, and therefore also that $\left[\left\{g^{\prime}\right\}\right]_{s} \subseteq[\{g\}]_{s}$.

For the reverse inclusion let

$$
g^{\prime \prime}\left(x_{1}, \ldots, x_{n}\right)=g^{\prime}\left(\pi_{1}^{n}\left(x_{1}, \ldots, x_{n}\right), \ldots, \pi_{m}^{n}\left(x_{1}, \ldots, x_{n}\right)\right),
$$

and let $g^{\prime \prime \prime}=g^{\prime \prime} \circ \sigma^{-1}$. Clearly, $g$ equivalent to $g^{\prime \prime \prime}$ except that the arguments $x_{m+1}, \ldots, x_{n}$ of $g^{\prime \prime \prime}$ can take arbitrary values. Hence, $g$ is a subfunction of $g^{\prime \prime \prime}$. From this it follows that $g \in\left[\left\{g^{\prime \prime \prime}\right\}\right]_{S}$ and, reasoning as above, $g \in\left[\left\{g^{\prime}\right\}\right]_{S}$, hence $[\{g\}]_{S} \subseteq\left[\left\{g^{\prime}\right\}\right]_{s}$. 
The notions of contraction and stretching will allow us to bound the arities of the functions needed to characterise a strong partial clone. Dually, we now introduce the notion of a $k$-restriction, which will allow us to bound the size of the domain of these functions.

Definition 5.5 ( $k$-restriction). Let $f$ be an $n$-ary partial function, and $k$ be an integer with $\mid$ domain $(f) \mid \geq k$. An $n$-ary function $g$ is said to be a $k$-restriction of $f$ if $g$ is a subfunction of $f$ and $|\operatorname{domain}(g)|=k$ holds.

For a partial function $f$ and an integer $k$, we write $\mathcal{R}_{k}(f)$ for the set of all functions that are a $k$-restriction of $f$ if $\mid$ domain $(f) \mid \geq k$ holds, and otherwise we define $\mathcal{R}_{k}(f)=\varnothing$. If $F$ is a set of partial functions, then $\mathcal{R}_{k}(F)$ denotes $\bigcup_{f \in F} \mathcal{R}_{k}(f)$. The following lemma, which relates partial polymorphisms with their restrictions and contractions, is the cornerstone of our study.

Lemma 5.6. Let $R$ be a relation, let $\kappa \geq \operatorname{ar}(R)$ and $n>\mu \geq$ \#tuples $(R)$, and let $f$ be an $n$-ary partial function. Then $f \in \operatorname{pPol}(\{R\})$ holds if and only if $\mathcal{C}_{\mu}\left(\mathcal{R}_{\kappa^{\prime}}(f)\right) \subseteq \operatorname{pPol}(\{R\})$ holds for all $\kappa^{\prime} \leq \kappa$.

Proof. First assume $f \in \operatorname{pPol}(\{R\})$. Then $g \in \operatorname{pPol}(\{R\})$ holds for every subfunction $g$ of $f$, and all $\mu$-contractions $g^{\prime}$ of such a $g$ are in $\operatorname{pPol}(\{R\})$ by Lemma 5.4 . For the other direction, towards contradiction assume $f \notin$ $\operatorname{pPol}(\{R\})$. Then there is a sequence of tuples $t_{1}, \ldots, t_{n} \in R$ with

$$
f\left(t_{1}, \ldots, t_{n}\right)=\left(f\left(t_{1}[1], \ldots, t_{n}[1]\right), \ldots, f\left(t_{1}[\operatorname{ar}(R)], \ldots, t_{n}[\operatorname{ar}(R)]\right)\right) \notin R .
$$

Note that at least $n-\mu$ of these tuples must be repeated since we assume $n>\mu \geq$ \#tuples $(R)$. Let $\lambda \leq \operatorname{ar}(R) \leq \kappa$ be the number of distinct tuples in $\operatorname{Cols}\left(t_{1}, \ldots, t_{n}\right)$, and let $g$ be the $\lambda$-restriction $g$ of $f$ defined only on these tuples. Since all but at most $\mu$ tuples are repeated in $t_{1}, \ldots, t_{n}$ there exists at least one $\mu$-contraction $g^{\prime}$ of $g$ and $t_{i_{1}}, \ldots t_{i_{\mu}} \subseteq\left\{t_{1}, \ldots, t_{n}\right\}$ such that

$$
g^{\prime}\left(t_{i_{1}}, \ldots, t_{i_{\mu}}\right)=g\left(t_{1}, \ldots, t_{n}\right)=f\left(t_{1}, \ldots, t_{n}\right) \notin R .
$$

This contradicts that all $\mu$-contractions of all $\lambda$-restrictions of $f$ are in $\operatorname{pPol}(\{R\})$.

We immediately get the following generalisation to constraint languages (instead of single relations).

Corollary 5.7. Let $S$ be a finite constraint language, let $\kappa \geq \operatorname{ar}(S), n>\mu \geq$ \#tuples $(S)$, and let $f$ be an $n$-ary partial function. Then $f \in \operatorname{pPol}(S)$ if and only if $\mathcal{C}_{\mu}\left(\mathcal{R}_{\mathcal{K}^{\prime}}(f)\right) \subseteq \operatorname{pPol}(S)$ for all $\kappa^{\prime} \leq \kappa$.

Proof. Assume $f \in \operatorname{pPol}(S)$, and let $R \in S$, hence $f \in \operatorname{pPol}(\{R\})$. Let $\kappa^{\prime} \leq \kappa$, and let $g^{\prime}$ be a $\mu$-contraction of a $\kappa^{\prime}$-restriction $g$ of $f$. By Lemma $5.6 g^{\prime}$ is in $\operatorname{pPol}(\{R\})$. Since $R$ was arbitrarily chosen in $S$ we get $g^{\prime} \in \operatorname{pPol}(S)$. For the other direction, let $f$ be as in the claim, and let $R \in S$, hence $\mathcal{C}_{\mu}\left(\mathcal{R}_{\mathcal{K}^{\prime}}(f)\right) \subseteq$ $\operatorname{pPol}(\{R\})$. By Lemma 5.6 again we get $f \in \operatorname{pPol}(\{R\})$, and since $R$ was arbitrarily chosen in $S$ we conclude that $f \in \operatorname{pPol}(S)$. 
Example 5.8. Let $f$ be the ternary partial function defined by $f(0,0,1)=0$, $f(0,1,0)=0, f=(1,0,0)=1$, and $f(1,1,1)=0$. Observe that $f$ restricted to $\{(0,0,1),(0,1,0),(1,0,0)\}$ is a subfunction of $\pi_{1}^{3}$. To check that $f \in \operatorname{pPol}\left(\left\{R_{1 / 3}\right\}\right)$ one only has to verify that the contractions of all 1, 2-, and 3-restrictions of $f$ are in $\operatorname{pPol}\left(\left\{R_{1 / 3}\right\}\right)$. Since any restriction not defined on $(1,1,1)$ is a projection, we only need to check that all restrictions containing $(1,1,1)$ preserve $R_{1 / 3}$. We do this in turn. First let $g_{1}$ be the 1-restriction defined only on $(1,1,1)$. This function is 1-contractable to $g_{1}^{\prime}(1)=0$ and we easily see that $g^{\prime}$ preserves $R_{1 / 3}$, hence so does $g_{1}$ by Lemma 5.4. For 2-restrictions let, e.g., $g_{2}(0,0,1)=0$ and $g_{2}(1,1,1)=0$, and let $g_{2}^{\prime}(0,1)=0, g_{2}^{\prime}(1,1)=0$ be its only 2 -contraction. This function also preserves $R_{1 / 3}$ since its application on two tuples from $R_{1 / 3}$ will always be undefined. As for 3-restrictions let, e.g., $g_{3}(0,0,1)=0, g_{3}(0,1,0)=0$, and $g_{3}(1,1,1)=0$. This function is not 1 - or 2 -contractable, but it preserves $R_{1 / 3}$ since, for instance, $g_{3}((0,0,1),(0,1,0),(1,0,0))$ is undefined, and if one tuple from $R_{1 / 3}$ is repeated (as in the case of $\left.g_{3}((0,0,1),(0,0,1),(0,1,0))\right)$, then $g_{3}$ is also undefined since there is at least one application of $g_{3}(0,0,0)$, which is undefined. Hence $f$ preserves $R_{1 / 3}$ since all contractions of its 1-, 2- and 3-restrictions preserve $R_{1 / 3}$.

Corollary 5.7 implies that the partial polymorphisms of a finite constraint language can be derived from those with bounded arity and domain (with bounds depending on the language). We now define these to constitute the bounded base of the constraint language.

Definition 5.9 (bounded base). Let $S$ be a finite constraint language with $\kappa=\operatorname{ar}(S)$ and $\mu=\#$ tuples $(S)$. The bounded base of $S, B(S)$, is defined to be

$$
B(S)=\{f|f \in \operatorname{pPol}(S), \operatorname{ar}(f) \leq \mu,| \operatorname{domain}(f) \mid \leq \kappa\} .
$$

The following theorem shows that representing languages by their bounded bases suffices to distinguish languages with different expressivity.

Theorem 5.10. Let $S, S^{\prime}$ be finite and column-irredundant constraint languages with $B(S)=B\left(S^{\prime}\right)$. Then $\operatorname{pPol}(S)=\operatorname{pPol}\left(S^{\prime}\right)$ holds.

Proof. If $\operatorname{ar}(S)=\operatorname{ar}\left(S^{\prime}\right)$ and \#tuples $(S)=$ \#tuples $\left(S^{\prime}\right)$ then the result follows directly from Corollary 5.7. Now assume towards contradiction $\operatorname{pPol}(S) \neq$ $\operatorname{pPol}\left(S^{\prime}\right)$ and $\kappa=\operatorname{ar}(S) \neq \kappa^{\prime}=\operatorname{ar}\left(S^{\prime}\right)$, and by symmetry $\kappa<\kappa^{\prime}$.

Note that $B\left(S^{\prime}\right)$ contains no function $f$ with $\mid$ domain $(f) \mid=\kappa^{\prime}$, since by definition $B(S)$ does not contain any function with a domain of size more than $\kappa$, and such an $f$ would separate $B\left(S^{\prime}\right)$ from $B(S)$, contradicting our assumption. Now since $S^{\prime}$ is column-irredundant we have $\kappa^{\prime} \leq|D|^{\mu^{\prime}}$ for $\mu^{\prime}=$ \#tuples $\left(S^{\prime}\right)$, and obviously all $\mu^{\prime}$-ary projections have $\kappa^{\prime}$-restrictions. These are in $B\left(S^{\prime}\right)$ by definition of a bounded base, yielding a contradiction.

Finally assume $\mu=$ \#tuples $(S) \neq \mu^{\prime}=$ \#tuples $\left(S^{\prime}\right)$, and by symmetry that $\mu<\mu^{\prime}$. Then as above we get from $B(S)=B\left(S^{\prime}\right)$ that $B\left(S^{\prime}\right)$ contains 
no $\mu^{\prime}$-ary function. But any 1-restriction of an $\mu^{\prime}$-ary projection has to be included in $B\left(S^{\prime}\right)$, which results in a contradiction.

It may be the case that two different languages generate the same weak partial co-clone but have different bounded bases because their arities or numbers of tuples are different, but as we show in Section 5.4. we can still compare their expressivity using bounded bases.

\subsection{Closure of Bounded Bases}

In this section we show how, for a finite constraint language $S$, the whole strong partial clone $\mathrm{pPol}(S)$ can be recovered from the functions in the finite base $B(S)$, which is done using a notion of closure stronger than functional composition. With this we investigate how bounded bases can be used to compare the expressivity of finite constraint languages. We first need some preliminary definitions to cope with the fact that the bounded base is a finite set.

Definition 5.11 (covering). Let $G$ be a set of $n$-ary partial functions, and let $k \geq 1$. Then $G$ is said to be $k$-covering if for all $U \subseteq \cup_{g \in G}$ domain $(g)$ satisfying $|U| \leq k$, there is a function $g \in G$ with domain $(g)=U$.

We say that a set of $n$-ary partial functions $G$ is consistent if for all $g, g^{\prime} \in G$ and all $\left(x_{1}, \ldots, x_{n}\right) \in \operatorname{domain}(g) \cap \operatorname{domain}\left(g^{\prime}\right)$, it holds that $g\left(x_{1}, \ldots, x_{n}\right)=$ $g^{\prime}\left(x_{1}, \ldots, x_{n}\right)$. Consistency ensures that $g$ can be chosen arbitrarily in the next definition.

Definition 5.12 (union). Let $G$ be a consistent set of $n$-ary partial functions. The union of $G$, written $\mathrm{u}(G)$, is the $n$-ary partial function defined by $\mathrm{u}(G)\left(x_{1}, \ldots, x_{n}\right)=g\left(x_{1}, \ldots, x_{n}\right)$ for all $\left(x_{1}, \ldots, x_{n}\right)$ such that some $g \in G$ is defined on $\left(x_{1}, \ldots, x_{n}\right)$, and undefined for other tuples.

Given a function $f$ and $k \geq 1$, we define the $k$-covering of $f$ as the set of functions $\mathcal{G}_{k}(f)=\bigcup_{k^{\prime}=1}^{k} \mathcal{R}_{k^{\prime}}(f)$. Observe that $\mathrm{u}\left(\mathcal{G}_{k}(f)\right)=f$ holds. Next recall that $\mathcal{S}_{n}(F)$ denotes the set of all functions that are an $n$-stretching of some function in $F$, and that $\mathcal{U}_{n}^{m}$ denotes the set of all $n$-ary partial functions that are uncontractable to arity $m$. We are now ready to define our notion of closure.

Definition 5.13 (closure). Let $F$ be a finite set of partial functions with $m=$ $\operatorname{ar}(F)$. Let $k \geq 1, n \geq 1$ be integers. The $k, n$-closure of $F$, written $C l_{k, n}(F)$, is the set of $n$-ary functions defined by

$$
C l_{k, n}(F)=\{\mathrm{u}(G) \mid G \subseteq F, \operatorname{ar}(G)=n, G \text { is } k \text {-covering and consistent }\}
$$

for $n \leq m$, and

$$
C l_{k, n}(F)=\left\{\mathrm{u}(G) \mid G \subseteq \mathcal{S}_{n}(F) \cup \mathcal{U}_{n}^{m}, G \text { is } k \text {-covering and consistent }\right\}
$$


for $n>m$. The $k$-closure of $F$ is defined to be

$$
C l_{k}(F)=\bigcup_{n=1}^{\infty} C l_{k, n}(F) .
$$

Example 5.14. As a simple example let $R_{0}=\{(0)\}$. From Table 2.1 in Chapter 2. Table 3.1 in Chapter 3 and Table 4.1 in Chapter 4 it is easy to see that $\operatorname{pPol}\left(\left\{R_{0}\right\}\right)=\left[\left\{x_{1} \wedge x_{2}, x_{1} \oplus x_{2}\right\}\right]_{s}$. The bounded base $B\left(\left\{R_{0}\right\}\right)$ consists of all unary $f \in \operatorname{pPol}\left(\left\{R_{0}\right\}\right)$ satisfying $|\operatorname{domain}(f)|=1$. Thus $B\left(\left\{R_{0}\right\}\right)=\left\{f_{1}, f_{2}, f_{3}\right\}$ where $f_{1}, f_{2}, f_{3}$ are defined as $f_{1}(0)=0, f_{2}(1)=1$, $f_{3}(1)=0$, and undefined otherwise. To exemplify 1,2-closure we see that $x_{1} \wedge x_{2}=\mathrm{u}(G)\left(x_{1}, x_{2}\right)$ where $G=\left\{g_{1}, g_{2}, g_{3}, g_{4}\right\}$ and $g_{1}(0,0)=0$, $g_{2}(0,1)=0, g_{3}(1,0)=0, g_{4}(1,1)=1$, and undefined for all other values. Since $g_{1} \in \mathcal{S}_{2}\left(\left\{f_{1}\right\}\right), g_{4} \in \mathcal{S}_{2}\left(\left\{f_{2}\right\}\right)$, and $g_{2}, g_{3} \in \mathcal{U}_{2}^{1}$, it follows that $x_{1} \wedge x_{2} \in C l_{1,2}\left(\left\{f_{1}, f_{2}, f_{3}\right\}\right)$.

Note that we slightly abuse the term 'closure', since the $k$-closure operator fails to satisfy all properties normally associated with closure operators. For example, it does not hold that $C l_{k}(F)=C l_{k}\left(C l_{k}(F)\right)$ since $k$-closure is only defined for finite sets of partial functions. With the notion of $k$-closure in hand, we can now show that it captures exactly what we want, namely, that the $\operatorname{ar}(S)$-closure of the bounded base of $S$ is exactly $\mathrm{pPol}(S)$.

Theorem 5.15. Let $S$ be a finite constraint language. Then $C l_{\operatorname{ar}(S)}(B(S))=$ $\operatorname{pPol}(S)$ holds.

Proof. Write $\kappa=\operatorname{ar}(S)$ and $\mu=$ \#tuples $(S)$, and first let $f \in C l_{\kappa}(B(S))$ be an $n$-ary partial function. For $n \leq \mu$, by definition of closure the $\kappa$-covering $\mathcal{G}_{\kappa}(f)$ of $f$ satisfies $\mathcal{G}_{\kappa}(f) \subseteq B(S) \subseteq \operatorname{pPol}(S)$. Since all relations in $S$ are at most $\kappa$-ary, any application of $f$ to tuples from such a relation is in fact an application of some $f^{\prime}$ in the $\kappa$-covering of $f$, hence $f$ preserves $S$. Now for $n>\mu$ we have by definition of closure that for all $\kappa^{\prime} \leq \kappa$ and all $\kappa^{\prime}$-restrictions $g$ of $f$, either $g$ is uncontractable to arity $\mu$ or $g$ is the stretching of a function $g^{\prime}$ in $B(S) \subseteq \mathrm{pPol}(S)$. In the latter case all $\mu$-contractions of $g$ are in $\mathrm{pPol}(S)$ by Lemma 5.4. and this also holds vacuously in the former case. Hence, we get $f \in \operatorname{pPol}(S)$ by Corollary 5.7

Conversely, let $f \in \operatorname{pPol}(S)$ be an $n$-ary function. For $n \leq \mu$, by definition the $\kappa$-covering $\mathcal{G}_{\kappa}(f)$ of $f$ consists of functions of arity $n$ that are defined on at most $\kappa$ tuples, and which are in $\operatorname{pPol}(S)$ as subfunctions of $f$. It follows that $\mathcal{G}_{\mathcal{K}}(f) \subseteq B(S)$ and hence, $f=\mathrm{u}\left(\mathcal{G}_{\kappa}(f)\right)$ is in $C l_{\mathcal{K}}(B(S))$. Finally, for $n>\mu$, let $\kappa^{\prime} \leq \kappa$ and let $g$ be a $\kappa^{\prime}$-restriction of $f$, that is, let $g$ be a function in the $\kappa$-covering $\mathcal{G}_{\kappa}(f)$ of $f$. We get $\mathcal{C}_{\mu}(g) \subseteq \operatorname{pPol}(S)$ from Corollary 5.7 and hence either $g \in \mathcal{U}_{n}^{\mu}$ holds or $g$ has at least one $\mu$-contraction $g^{\prime}$, which is in $\mathrm{pPol}(S)$ and hence in $B(S)$. In the latter case $g$ is a stretching of $g^{\prime}$, and finally, $\mathcal{G}_{\kappa}(f) \subseteq \mathcal{S}_{n}(B(S)) \cup \mathcal{U}_{n}^{\mu}$, which concludes the proof.

To exemplify this result, let $R_{0}$ and $f_{1}, f_{2}, f_{3}$ be defined as in Example 5.14 From Theorem 5.15 we get $C_{1}\left(\left\{f_{1}, f_{2}, f_{3}\right\}\right)=\operatorname{pPol}\left(\left\{R_{0}\right\}\right)$, i.e., the set of all 
total or partial functions that are 0-reproducing. The proof of Theorem 5.15 also makes it clear why we need the sets $\mathcal{U}_{n}^{\mu}$ of uncontractable functions.

\subsection{Determining Bounded Bases of Boolean Constraint Languages}

In this section we turn to the problem of determining bounded bases of Boolean constraint languages. We start with a general construction for retrieving the partial polymorphisms of the language obtained from another one by allowing some arguments to be negated in constraint applications, and then determine bounded bases for two of the most studied languages corresponding to NP-complete SAT problems, namely MONOTONE 1 -IN- $\kappa$-SAT and $\kappa-S A T$.

\subsubsection{Sign Patterns}

It is natural to ask how the complexity of $\operatorname{CSP}(S)$ is influenced by negated arguments in constraint applications. To handle this we extend $S$ by additional relations, representing the cases where one or more arguments are negated. Recall from Section 2.3.2 in Chapter 2 that $R^{\left(s_{1}, \ldots, s_{\kappa}\right)}$ denotes the relation $\left\{\left(x_{1}^{s_{1}}, \ldots, x_{\mathcal{K}}^{s_{\mathcal{K}}}\right) \mid\left(x_{1}, \ldots, x_{\mathcal{K}}\right) \in R\right\}$, with $x_{i}^{+}=x_{i}$ and $x_{i}^{-}=\overline{x_{i}}$. As a shorthand we in this section write $\bar{R}=R^{(-, \ldots,-)}$. Interestingly, it is possible to describe $\operatorname{pPol}(\{\bar{R}\})$ and $\operatorname{pPol}(\{R, \bar{R}\})$ in terms of $\operatorname{pPol}(\{R\})$. For an $n$-ary Boolean partial function $f$, let the dual dual $(f)$ be defined by dual $(f)(\bar{u})=\overline{f(u)}$ for all $u \in \operatorname{domain}(f)$.

Theorem 5.16. Let $R$ be Boolean relation. Then $\operatorname{pPol}(\{\bar{R}\})=\{\operatorname{dual}(f) \mid f \in$ $\operatorname{pPol}(\{R\})\}$ and $\operatorname{pPol}(\{R, \bar{R}\})=\{f \mid f, \operatorname{dual}(f) \in \operatorname{pPol}(\{R\})\}$ hold.

Proof. For the first statement, first note $\bar{t} \in R \Longleftrightarrow t \in \bar{R}$. Let $f \in$ $\operatorname{pPol}(\{R\})$ be an $n$-ary partial function, and assume towards contradiction that $\operatorname{dual}(f) \notin \operatorname{pPol}(\{\bar{R}\})$. Then there exists $t_{1}, \ldots, t_{n} \in \bar{R}$ with $\operatorname{dual}(f)\left(t_{1}, \ldots, t_{n}\right)=t \notin \bar{R}$. But if $\operatorname{dual}(f)\left(t_{1}, \ldots, t_{n}\right)$ is defined then $f\left(\overline{t_{1}}, \ldots, \overline{t_{n}}\right)=\bar{t}$ is defined, and by assumption $\bar{t} \in R$. By definition of a dual function this means that $\operatorname{dual}(f)\left(t_{1}, \ldots, t_{n}\right)=\overline{f\left(\overline{t_{1}}, \ldots, \overline{t_{n}}\right)}=\overline{\bar{t}}=t$ must be in $\bar{R}$, a contradiction. Hence, $\operatorname{dual}(f)$ is in $\operatorname{pPol}(\{\bar{R}\})$. The converse follows through entirely analogous arguments.

The second statement follows directly from the first one since by definition we have $f \in \operatorname{pPol}(\{R, \bar{R}\}) \Longleftrightarrow f \in \operatorname{pPol}(\{R\}) \wedge f \in \operatorname{pPol}(\{\bar{R}\})$.

The cases when one or more, but not all, arguments are negated are not as straightforward, and we need a few additional definitions. First, call a partial function $f$ self-dual if for all $u \in \operatorname{domain}(f)$ we have $\bar{u} \in \operatorname{domain}(f)$ and $f(\bar{u})=\overline{f(u)}$. Second, call a language $S$ closed under sign patterns if for all $R \in S$ and $s_{1}, \ldots, s_{\operatorname{ar}(R)} \in\{+,-\}$, the relation $R^{\left(s_{1}, \ldots, s_{\operatorname{ar}(R)}\right)}$ is also in $S$. 
Theorem 5.17. Let $R$ be a $\kappa$-ary Boolean relation. If $f$ is in $\operatorname{pPol}(\{R\})$ and $f$ is self-dual, then $f$ is in $\operatorname{pPol}\left(\left\{R^{\left(s_{1}, \ldots, s_{\kappa}\right)}\right\}\right)$ for every sign pattern $\left(s_{1}, \ldots, s_{\kappa}\right)$.

Proof. Assume towards contradiction that $f \notin \operatorname{pPol}\left(\left\{R^{\left(s_{1}, \ldots, s_{\kappa}\right)}\right\}\right)$. Let $n=$ $\operatorname{ar}(f)$, and let $t_{1}, \ldots, t_{n} \in R^{\left(s_{1}, \ldots, s_{\kappa}\right)}$ be such that $f\left(t_{1}, \ldots, t_{n}\right)$ is defined but $f\left(t_{1}, \ldots, t_{n}\right)=t \notin R^{\left(s_{1}, \ldots, s_{\kappa}\right)}$. Let $t_{1}^{\prime}, \ldots, t_{n}^{\prime} \in R$ be the corresponding tuples of $R$, i.e., $t_{i}^{\prime}[j]=t_{i}[j]$ for $s_{j}=+$ and $t_{i}^{\prime}[j]=\overline{t_{i}[j]}$ for $s_{j}=-$, and similarly let $t^{\prime}$ be the tuple corresponding to $t$. Then $t_{1}^{\prime}, \ldots, t_{n}^{\prime} \in R$ holds by definition of $R^{\left(s_{1}, \ldots, s_{\kappa}\right)}$, and $f\left(t_{1}^{\prime}, \ldots, t_{n}^{\prime}\right)$ is defined since $f$ is self-dual, but $f\left(t_{1}^{\prime}, \ldots, t_{n}^{\prime}\right)=t^{\prime}$ is not in $R$ by definition of $R^{\left(s_{1}, \ldots, s_{\kappa}\right)}$, again, contradicting $f \in \operatorname{pPol}(\{R\})$.

Theorem 5.18. Let $S$ be a language closed under sign patterns, and let $f \in \operatorname{pPol}(S)$ with $u \in \operatorname{domain}(f)$ but $\bar{u} \notin \operatorname{domain}(f)$. Define $f^{\prime}$ by $f^{\prime}(\bar{u})=\overline{f(u)}$, and $f^{\prime}\left(u^{\prime}\right)=f\left(u^{\prime}\right)$ for every $u^{\prime} \in \operatorname{domain}(f)$. Then $f^{\prime} \in \operatorname{pPol}(S)$.

Proof. Let $n=\operatorname{ar}(f)$. Assume towards contradiction that for some $R \in S$ of arity $\operatorname{ar}(R)=\kappa$ we have $t_{1}, \ldots, t_{n} \in R, f^{\prime}\left(t_{1}, \ldots, t_{n}\right)$ defined, and $f^{\prime}\left(t_{1}, \ldots, t_{n}\right) \notin R$. Let $I \subseteq\{1, \ldots, \kappa\}$ be the set of all indices $i$ for which $\left(t_{1}[i], \ldots, t_{n}[i]\right)=\bar{u}$; note that $I \neq \varnothing$, as otherwise we have a witness against $f \in \operatorname{pPol}(S)$. Let $R^{\prime} \in S$ be $R$ negated exactly in arguments $I$ and for each $1 \leq j \leq n$ let $t_{j}^{\prime}$ be $t_{j}$ negated in positions $I$. Then $f\left(t_{1}^{\prime}, \ldots, t_{n}^{\prime}\right)$ is defined (and uses the tuple $u \in \operatorname{domain}(f)$ in place of $\bar{u}$ ), and $t_{1}^{\prime}, \ldots, t_{n}^{\prime} \in R^{\prime}$, but $f\left(t_{1}^{\prime}, \ldots, t_{n}^{\prime}\right) \notin R^{\prime}$ (since $f\left(t_{1}^{\prime}, \ldots, t_{n}^{\prime}\right)$ differs from $f^{\prime}\left(t_{1}, \ldots, t_{n}\right)$ in exactly the positions $I$ ). This contradicts the assumption $f \in \operatorname{pPol}(S)$.

Given two additional assumptions, we can strengthen this further. Call a constraint language $S$ closed under fixing arguments if for all $R \in S, 1 \leq$ $i \leq \operatorname{ar}(R), c \in\{0,1\}$, the relation $R_{x_{i} \leftarrow c}=\left\{\left(x_{1}, \ldots, x_{i-1}, c, x_{i+1}, \ldots, x_{\operatorname{ar}(R)}\right) \mid\right.$ $\left.\left(x_{1}, \ldots, x_{i-1}, x_{i}, x_{i+1}, \ldots, x_{\operatorname{ar}(R)}\right) \in R\right\}$ is in $S$.

Theorem 5.19. Let $R$ be a Boolean relation, $S$ be the closure of $\{R\}$ under sign patterns, and let $S^{\prime}$ be the closure of $S$ under fixing arguments. If $\operatorname{Pol}(S)=I_{2}$ then $\operatorname{pPol}\left(S^{\prime}\right)=\left\{f^{\prime} \mid f^{\prime}\right.$ is a subfunction of some $f \in$ $\operatorname{pPol}(\{R\}), f$ is 0 -reproducing, 1-reproducing, and self-dual $\}$.

Proof. We show the result in two phases, first showing that $\operatorname{pPol}\left(S^{\prime}\right)=\left\{f^{\prime} \mid\right.$ $f^{\prime}$ is a subfunction of $f \in \operatorname{pPol}(S), f$ is 0-reproducing and 1-reproducing $\}$, then showing that this set, in turn, is characterised from $\operatorname{pPol}(\{R\})$ as described. The first phase is essentially applications of Nordh and Zanuttini [NZ09], but we include proof sketches here for a self-contained presentation. Through the remainder of this proof let $n=\operatorname{ar}(f)$. First, we need to establish that there is no $f \in \operatorname{pPol}\left(S^{\prime}\right)$ with $f\left(\overrightarrow{0}^{n}\right)=1$ or $f\left(\overrightarrow{1}^{n}\right)=0$. For this, let $R^{\prime} \in S^{\prime}$ with $\overrightarrow{0}^{\text {ar }\left(R^{\prime}\right)} \in R^{\prime}$ and $\overrightarrow{1} \operatorname{ar}\left(R^{\prime}\right) \notin R^{\prime}$; observe that $S^{\prime}$ is closed under sign patterns, hence such a relation $R^{\prime}$ exists unless every $R^{\prime} \in S^{\prime}$ is self-dual (i.e., $\bar{t} \in R^{\prime}$ for every $t \in R^{\prime}$ ), which would contradict $\langle S\rangle=\mathrm{BR}$, as it means that the total unary polymorphism $\bar{x}$ preserves $S^{\prime}$. Hence $R^{\prime}$ witnesses that $f\left(\overrightarrow{0}^{n}\right) \neq 1$ for every $f \in \operatorname{pPol}\left(S^{\prime}\right)$, and $\overline{R^{\prime}}$ witnesses that $f\left(\overrightarrow{1}^{n}\right) \neq 0$. 
Next, we show that every $f \in \operatorname{pPol}\left(S^{\prime}\right)$ is a subfunction of some $f^{\prime} \in$ $\operatorname{pPol}\left(S^{\prime}\right)$ with $\overrightarrow{0}^{n}, \overrightarrow{1}^{n} \in \operatorname{domain}\left(f^{\prime}\right)$ and $f^{\prime}\left(\overrightarrow{0}^{n}\right)=0, f^{\prime}\left(\overrightarrow{1}^{n}\right)=1$, i.e., that every $f \in \operatorname{pPol}\left(S^{\prime}\right)$ can be extended by setting $f\left(\overrightarrow{0}^{n}\right)=0$ and $f\left(\overrightarrow{1}^{n}\right)=1$, and the resulting function $f^{\prime}$ is also a partial polymorphism of $S^{\prime}$. Assume to the contrary that for some $R^{\prime} \in S^{\prime}$ and tuples $t_{1}, \ldots, t_{n} \in R^{\prime}$, we have $f^{\prime}\left(t_{1}, \ldots, t_{n}\right)$ defined and $f^{\prime}\left(t_{1}, \ldots, t_{n}\right) \notin R^{\prime}$. Then we must have that $f\left(t_{1}, \ldots, t_{n}\right)$ is undefined. However, the only tuples in domain $\left(f^{\prime}\right) \backslash$ domain $(f)$ are the constants $\overrightarrow{0}^{n}$ and $\overrightarrow{1}^{n}$. Thus, the tuples $t_{1}, \ldots, t_{n}$ as well as the function output $f^{\prime}\left(t_{1}, \ldots, t_{n}\right)$ define a pattern of fixed arguments in $R^{\prime}$, hence there is some further relation $R^{\prime \prime} \in S^{\prime}$ corresponding to fixing this pattern in $R^{\prime}$, with tuples $t_{1}^{\prime}, \ldots, t_{n}^{\prime} \in R^{\prime \prime}$ such that $f\left(t_{1}^{\prime}, \ldots, t_{n}^{\prime}\right)$ is defined, hence $f\left(t_{1}^{\prime}, \ldots, t_{n}^{\prime}\right)=f^{\prime}\left(t_{1}^{\prime}, \ldots, t_{n}^{\prime}\right) \notin R^{\prime \prime}$, contradicting that $f \in \operatorname{pPol}\left(S^{\prime}\right)$. This shows that every $f \in \operatorname{pPol}\left(S^{\prime}\right)$ is a subfunction of some $f^{\prime} \in \operatorname{pPol}\left(S^{\prime}\right)$ with $f^{\prime}\left(\overrightarrow{0}^{n}\right)=0$ and $f^{\prime}\left(\overrightarrow{1}^{n}\right)=1$.

For the final part of the first phase, we show that if $f$ is a partial function with $\overrightarrow{0}^{n}, \overrightarrow{1}^{n} \in \operatorname{domain}(f)$ and $f\left(\overrightarrow{0}^{n}\right)=0, f\left(\overrightarrow{1}^{n}\right)=1$, then $f \in \operatorname{pPol}(S)$ if and only if $f \in \operatorname{pPol}\left(S^{\prime}\right)$. One direction is trivial: as $S \subseteq S^{\prime}$ we have $\operatorname{pPol}\left(S^{\prime}\right) \subseteq \operatorname{pPol}(S)$, so it remains to show that assuming $f \in \operatorname{pPol}(S)$, we get $f \in \operatorname{pPol}\left(S^{\prime}\right)$. Assume towards contradiction $f \notin \operatorname{pPol}\left(S^{\prime}\right)$. Then there is some relation $R^{\prime} \in \operatorname{pPol}\left(S^{\prime}\right)$ with $t_{1}, \ldots, t_{n} \in R^{\prime}$ and $f\left(t_{1}, \ldots, t_{n}\right)$ defined, but $f\left(t_{1}, \ldots, t_{n}\right) \notin R^{\prime}$. Since $R^{\prime}$ is obtained from fixing arguments in some relation $R^{\prime \prime} \in S$, it must be that the tuples $t_{1}, \ldots, t_{n}$ can be extended, by inserting positions fixed to 0 and/or 1 , to some tuples $t_{1}^{\prime}, \ldots, t_{n}^{\prime}$ such that $t_{1}^{\prime}, \ldots, t_{n}^{\prime} \in$ $R^{\prime \prime}$ and either $f\left(t_{1}^{\prime}, \ldots, t_{n}^{\prime}\right) \in R^{\prime \prime}$ or $f\left(t_{1}^{\prime}, \ldots, t_{n}^{\prime}\right)$ is undefined. However, the latter is impossible, as the only columns in $\operatorname{Cols}\left(t_{1}^{\prime}, \ldots, t_{n}^{\prime}\right)$ not present in $\operatorname{Cols}\left(t_{1}, \ldots, t_{n}\right)$ are $\overrightarrow{0}^{n}$ and/or $\overrightarrow{1}^{n}$, and we have $\overrightarrow{0}^{n}, \overrightarrow{1}^{n} \in \operatorname{domain}(f)$. Thus we have $f\left(t_{1}^{\prime}, \ldots, t_{n}^{\prime}\right) \in R^{\prime \prime}$. But since $f\left(\overrightarrow{0}^{n}\right)=0$ and $f\left(\overrightarrow{1}^{n}\right)=1$, the output of $f\left(t_{1}^{\prime}, \ldots, t_{n}^{\prime}\right)$ matches the pattern of fixed arguments of $t_{1}^{\prime}, \ldots, t_{n}^{\prime}$, hence $f\left(t_{1}^{\prime}, \ldots, t_{n}^{\prime}\right) \in R^{\prime \prime}$ implies $f\left(t_{1}, \ldots, t_{n}\right) \in R^{\prime}$. We conclude that $f \in \operatorname{pPol}\left(S^{\prime}\right)$.

This concludes the first phase, and we have shown that $\mathrm{pPol}\left(S^{\prime}\right)$ consists exactly of subfunctions of

\section{$\{f \mid f \in \operatorname{pPol}(S), f$ is 0-reproducing and 1-reproducing $\}$,}

and we need to connect this set to $\operatorname{pPol}(\{R\})$. We proceed in the same three steps as before. First, let $f \in \operatorname{pPol}(S)$ with $f\left(\overrightarrow{0}^{n}\right)=0$ and $f\left(\overrightarrow{1}^{n}\right)=1$, and assume $\bar{u} \in \operatorname{domain}(f)$ and $f(u)=f(\bar{u})$ for some $u \in \operatorname{domain}(f)$. Consider the subfunction $f^{\prime}$ of $f$ with domain $\left(f^{\prime}\right)=\left\{\overrightarrow{0}^{n}, \overrightarrow{1}^{n}, u, \bar{u}\right\}$; then $\mid$ domain $\left(f^{\prime}\right) \mid=4$, and $f^{\prime}$ has a contraction $g$ which is a total binary function $g \in\{\wedge, \vee\}$. However, since $\mathrm{pPol}(S)$ is closed under taking subfunctions and contractions, we have $g \in \operatorname{pPol}(S)$, contradicting that $\langle S\rangle=\mathrm{BR}$.

For the second step, we may now invoke Theorem 5.18 to complete $f$ into a self-dual partial polymorphism of $S$, showing that every $f \in \operatorname{pPol}(S)$ with $f\left(\overrightarrow{0}^{n}\right)=0$ and $f\left(\overrightarrow{1}^{n}\right)=1$ is a subfunction of some $f \in \operatorname{pPol}(S)$ that is 0 -reproducing, 1-reproducing and self-dual. 
For the third and final step, we have as before that $f \in \operatorname{pPol}(S)$ trivially implies $f \in \operatorname{pPol}(\{R\})$, and by Theorem 5.17. for any $f \in \operatorname{pPol}(\{R\})$ that is self-dual we have $f \in \operatorname{pPol}(S)$. Hence, we have completed phase 2, establishing that the set

$$
\{f \mid f \in \operatorname{pPol}(S), f \text { is 0-reproducing and 1-reproducing }\}
$$

consists exactly of subfunctions of

$$
\{f \mid f \in \operatorname{pPol}(\{R\}), f \text { is 0-reproducing, 1-reproducing and self-dual }\} .
$$

Combining this with the result of phase 1 finishes the proof.

Theorem 5.16 and Theorem 5.19 provide sufficient conditions for when it is possible to describe $\operatorname{pPol}(S)$ through $\operatorname{pPol}(\{R\})$ if $S$ is a sign pattern expansion of $R$. Naturally this also holds for $B(S)$, which means that bounded bases for these languages can be expressed in a particularly simple form.

\subsubsection{1-IN- $\kappa-S A T$}

In this section we determine the bounded base of $R_{1 / \kappa}$. Recall from Section 2.3.2 in Chapter 2 that

$$
R_{1 / \kappa}=\left\{\left(x_{1}, \ldots, x_{\kappa}\right) \mid x_{1}, \ldots, x_{\kappa} \in\{0,1\}, \Sigma_{i=1}^{\kappa} x_{i}=1\right\},
$$

and note that \#tuples $\left(R_{1 / \kappa}\right)=\kappa$.

We give a general characterisation of the functions in the bounded base $B\left(\left\{R_{1 / \kappa}\right\}\right)$. For integers $\lambda, \kappa^{\prime}$ and a set of $\kappa^{\prime}$-ary Boolean tuples $U$ satisfying $|U|=\lambda$, we say that $U$ is an exact cover of $\left\{1, \ldots, \kappa^{\prime}\right\}$ if for all $i=1, \ldots, \kappa^{\prime}$, there is a unique $u \in U$ with $u[i]=1$. For instance, for $\lambda=2$ and $\kappa^{\prime}=3$, the set $U=\{(0,1,1),(1,0,0)\}$ is an exact cover of $\{1, \ldots, 3\}$, and for $\lambda=4$ and $\kappa^{\prime}=3$, the set $U=\{(0,0,0),(0,0,1),(0,1,0),(1,0,0)\}$ is an exact cover of $\{1, \ldots, 3\}$. Observe that $U$ is an exact cover of $\left\{1, \ldots, \kappa^{\prime}\right\}$ if and only if $U \backslash\{\overrightarrow{0}\}$ and $U \cup\{\overrightarrow{0}\}$ are exact covers, and that the size $\lambda=|U|$ of an exact cover $U$ of $\left\{1, \ldots, \kappa^{\prime}\right\}$ satisfies either $\lambda \leq \kappa^{\prime}+1$ or $\lambda \leq \kappa^{\prime}$, depending on whether $U$ contains $\overrightarrow{0}$. We then have the following two lemmas.

Lemma 5.20. Let $\kappa \geq \kappa^{\prime} \geq 1$. If $U \subseteq\{0,1\}^{\kappa^{\prime}}$ is an exact cover of $\left\{1, \ldots, \kappa^{\prime}\right\}$ with $|U|=\kappa$, then $U=\operatorname{ColsSet}\left(t_{1}, \ldots, t_{\mathcal{K}^{\prime}}\right)$ for some $t_{1}, \ldots, t_{\mathcal{K}^{\prime}} \in R_{1 / \kappa}$.

Proof. Write $U=\left\{u_{1}, \ldots, u_{\kappa}\right\}$. By definition of an exact cover, for all $1 \leq$ $i \leq \kappa^{\prime}$ the tuple $t_{i}=\left(u_{1}[i], \ldots, u_{\kappa}[i]\right)$ contains exactly one entry that is 1 . Hence $t_{i} \in R_{1 / \kappa}$ for all $1 \leq i \leq \mathcal{K}^{\prime}$, that is, $U=\operatorname{ColsSet}\left(t_{1}, \ldots, t_{\mathcal{K}^{\prime}}\right)$ for some $t_{1}, \ldots, t_{\mathcal{K}^{\prime}} \in R_{1 / \kappa}$.

Lemma 5.21. Let $\kappa>\kappa^{\prime} \geq 1$. Then $U \subseteq\{0,1\}^{\kappa^{\prime}}$ is an exact cover of $\left\{1, \ldots, \kappa^{\prime}\right\}$ with $\overrightarrow{0} \in U$ if and only if $\vec{U}=\operatorname{ColsSet}\left(t_{1}, \ldots, t_{\mathcal{K}^{\prime}}\right)$ for some $t_{1}, \ldots, t_{\mathcal{K}^{\prime}} \in R_{1 / \kappa}$. 
Proof. Assume $U=\left\{u_{1}, \ldots, u_{\kappa^{\prime}}\right\}$ is an exact cover of $\left[1, \ldots, \kappa^{\prime}\right]$ satisfying $\overrightarrow{0}^{\kappa^{\prime}} \in U$. By Lemma 5.20 each $\left(u_{1}[i], \ldots, u_{\mathcal{K}^{\prime}}[i]\right)$ corresponds to a tuple $\left(x_{1}, \ldots, x_{\kappa^{\prime}}\right) \in R_{1 / \kappa^{\prime}}$. Let $t_{i}=\left(y_{1}, \ldots, y_{\kappa}\right) \in R_{1 / \kappa}$ be the tuple satisfying $\left(x_{1}, \ldots, x_{\kappa^{\prime}}\right)=\left(y_{\kappa-\kappa^{\prime}}, \ldots, y_{\kappa}\right)$ and note that this implies $\left(y_{1}, \ldots, y_{\kappa-\kappa^{\prime}-1}\right)=$ $\overrightarrow{0}^{\kappa-\kappa^{\prime}-1}$. It is then readily seen that $\operatorname{ColsSet}\left(t_{1}, \ldots t_{\kappa^{\prime}}\right)=U$, which concludes the proof of the first direction. The other direction follows from the fact that for any $t_{1}, \ldots, t_{\mathcal{K}^{\prime}} \in R_{1 / \kappa}$ we have that $\operatorname{ColsSet}\left(t_{1}, \ldots, t_{\mathcal{K}^{\prime}}\right)$ is an exact cover of $\left[1, \ldots, \kappa^{\prime}\right]$ and that $\overrightarrow{0}^{\kappa^{\prime}} \in \operatorname{ColsSet}\left(t_{1}, \ldots, t_{\mathcal{K}^{\prime}}\right)$ since there exists at least one $1 \leq i \leq \kappa$ such that $\left(t_{1}[i], \ldots, t_{\kappa^{\prime}}[i]\right)=\overrightarrow{0}^{\kappa^{\prime}}$.

Theorem 5.22. Let $\kappa \geq 3$. Then the bounded base of $R_{1 / \kappa}$ is the set of all functions $f$ with $\kappa^{\prime}=\operatorname{ar}(f) \leq \kappa$ and defined on at most $\kappa$ tuples, that satisfy one of the following two conditions:

1. for all exact covers $U$ of $\left\{1, \ldots, \kappa^{\prime}\right\}$ contained in domain $(f)$ and with $\overrightarrow{0} \in U$ or $|U|=\kappa, f$ restricted to $U$ is a projection, or

2. $\overrightarrow{0} \in \operatorname{domain}(f)$, $\mid$ domain $(f) \mid=\kappa$, domain $(f)$ is an exact cover of $\left\{1, \ldots, \kappa^{\prime}\right\}, f(\overrightarrow{0})=1$, and $f\left(x_{1}, \ldots, x_{\kappa^{\prime}}\right)=0$ for all $\left(x_{1}, \ldots, x_{\kappa^{\prime}}\right) \in$ domain $(f) \backslash\{\overrightarrow{0}\}$.

Proof. Let $f$ be a function in $B\left(R_{1 / \kappa}\right)$. By definition of a bounded base, from $\operatorname{ar}\left(R_{1 / \kappa}\right)=$ \#tuples $\left(R_{1 / \kappa}\right)=\kappa$ we get $\operatorname{ar}(f)$, $\mid$ domain $(f) \mid \leq \kappa$. We now distinguish two cases for the tuple $\overrightarrow{0}$. First assume $\overrightarrow{0} \notin \operatorname{domain}(f)$ or $f(\overrightarrow{0})=0$, and let $U \subseteq$ domain $(f)$ be an exact cover of $\left\{1, \ldots, \kappa^{\prime}\right\}$ with $\overrightarrow{0} \in U$ or $|U|=\kappa$. Let $T$ be the sequence of tuples from $R_{1 / \kappa}$ satisfying $\operatorname{Cols} \operatorname{Set}(T)=U$. For $|U|=\kappa$ and $\overrightarrow{0} \notin U$, such $T$ exists by Lemma 5.20 , and it is easily seen that it has to contain each tuple of $R_{1 / \kappa}$ exactly once. For the case when $\overrightarrow{0} \in U$ such $T$ exists by Lemma 5.21 and $|\operatorname{domain}(f)| \leq \kappa$. Now because $f$ preserves $R_{1 / \kappa}$ by assumption, $f$ applied columnwise to $T$ must yield a tuple containing exactly one 1 . Hence for $|U|=\kappa$ and $\overrightarrow{0} \notin U$ it is clear that $f$ restricted to $U$ is a projection, and for $\overrightarrow{0} \in U$, using $f(\overrightarrow{0})=0$ we get that $f\left(x_{1}, \ldots, x_{\kappa^{\prime}}\right)$ must be 1 for some $\left(x_{1}, \ldots, x_{\kappa^{\prime}}\right) \in U \backslash\{\overrightarrow{0}\}$, hence again that $f$ restricted to $U$ is a projection. Finally, $f$ is in Case (1). Now assume $f(\overrightarrow{0})=1$ and that $f$ is not in Case (1). We first show that $\mid$ domain $(f) \mid=\kappa$ and domain $(f)$ is an exact cover of $\left\{1, \ldots, \kappa^{\prime}\right\}$. We first claim that domain $(f)$ does not contain an exact cover of $\left\{1, \ldots, \kappa^{\prime}\right\}$ of size less than $\kappa-1$. Indeed, otherwise it follows from Lemma 5.21 that there exists a sequence $T=\left(t_{i_{1}}, \ldots, t_{i_{\kappa^{\prime}}}\right)$ of tuples from $R_{1 / \kappa}$ satisfying $\operatorname{ColsSet}(T)=U \cup\{\overrightarrow{0}\}$ and such that $\overrightarrow{0}$ occurs at least twice a column. Hence $f$ applied columnwise to $T$ yields a tuple containing at least two 1 's and it follows that $f$ does not preserve $R_{1 / \kappa}$, a contradiction. With this in hand, since $f$ is not in Case (1), domain $(f)$ must contain an exact cover of $\left\{1, \ldots, \kappa^{\prime}\right\}$, hence one of size $\kappa-1$ or $\kappa$. Denote this exact cover by $U$. But for $|U|=\kappa-1, U$ cannot contain $\overrightarrow{0}$, since otherwise $U \backslash\{\overrightarrow{0}\}$ would be a cover of size less than $\kappa-1$, which we have just shown to be impossible. Now since we have $\overrightarrow{0} \in$ domain $(f)$ we get that $U \cup\{\overrightarrow{0}\}$ is an exact cover of size $\kappa$, as desired. Hence $\mid$ domain $(f) \mid=\kappa$ and domain $(f)$ is an exact cover of $\left\{1, \ldots, \kappa^{\prime}\right\}$. 
Letting $T$ be a sequence of tuples from $R_{1 / \kappa}$ with $\operatorname{ColsSet}(T)=\operatorname{domain}(f)$, we have that $f$ applied columnwise to $T$ must yield a tuple containing exactly one 1 . From $f(\overrightarrow{0})=1$ we conclude that $f$ is constantly 0 on domain $(f) \backslash\{\overrightarrow{0}\}$. Finally, $f$ is in Case (2).

We now show the reverse inclusion. Let $T$ be a sequence of $\kappa^{\prime}$ tuples from $R_{1 / \kappa}$ such that $f$ is defined on ColsSet $(T)$. We show that applying $f$ columnwise to $T$ yields a tuple in $R_{1 / \kappa}$, which is enough to show the claim. Since $T$ is a sequence of tuples from $R_{1 / \kappa}$ we have that $\operatorname{Cols} \operatorname{Set}(T)$ is an exact cover of $\left\{1, \ldots, \kappa^{\prime}\right\}$, which either contains $\overrightarrow{0}$ or is of size $\kappa$ (or both). Hence if $f$ is in Case (1), then its restriction to $U$ has to be a projection. Now, if $f$ is in Case (2), $\overrightarrow{0}$ cannot be repeated in $\operatorname{Cols}(T)$ since otherwise domain $(f) \backslash\{\overrightarrow{0}\}$ would be a proper superset of ColsSet $(T) \backslash\{\overrightarrow{0}\}$ (by $\mid$ domain $(f) \mid=\kappa$ ) and hence, could not be an exact cover of $\left\{1, \ldots, \kappa^{\prime}\right\}$. On the other hand, by definition of $R_{1 / \kappa}$, no other column can be repeated in $\operatorname{Cols}(T)$, hence from $|\operatorname{domain}(f)|=\kappa$ we conclude that $\operatorname{ColsSet}(T)=\operatorname{domain}(f)$. From the assumption it follows that $f$ applied columnwise to $T$ yields a tuple containing exactly one 1 , as desired.

We emphasise that a similar characterisation could be given for the whole set $\operatorname{pPol}\left(\left\{R_{1 / \kappa}\right\}\right)$, but using bounded bases makes the characterisation much simpler. For instance, Case (1) in the theorem encompasses the case when $f$ is a subfunction of a projection, but also the case when domain $(f)$ does not contain an exact cover of $\left\{1, \ldots, \kappa^{\prime}\right\}$. In this latter case $f$ can take arbitrary values, since there are no $t_{1}, \ldots, t_{\kappa^{\prime}} \in R_{1 / \kappa}$ such that $f\left(t_{1}, \ldots, t_{\kappa^{\prime}}\right)$ is defined. It also encompasses (through $\kappa, \kappa$-closure) the general case of functions $f$ (with $\mid$ domain $(f) \mid>\kappa)$ whose domain contains several exact covers over which $f$ is a projection, and other tuples which do not participate in any exact cover, and over which the value of $f$ is unconstrained.

\subsection{3 $\kappa-\mathrm{SAT}$}

In this section we use bounded bases to characterise the partial polymorphisms of $\Gamma_{\mathrm{SAT}}^{\kappa}$. The following theorem establishes that $B\left(\Gamma_{\mathrm{SAT}}^{\kappa}\right)$ is particularly simple, since it only consists of subfunctions of projections.

Theorem 5.23. Let $\kappa \geq 3$. Then the bounded base of $\kappa$-SAT is given by

$$
\begin{array}{r}
B\left(\Gamma_{\mathrm{SAT}}^{\kappa}\right)=\left\{f|| \text { domain }(f) \mid \leq \kappa, \operatorname{ar}(f) \leq 2^{\kappa}-1,\right. \\
\left.f \text { is a subfunction of some } \pi_{i}^{\operatorname{ar}(f)}\right\} .
\end{array}
$$

Proof. Since a partial projection function trivially preserves all relations it is clear that every partial projection $f$ with $|\operatorname{domain}(f)| \leq \kappa$ and $\operatorname{ar}(f) \leq$ $2^{\kappa}-1$ is included in $B\left(\Gamma_{\mathrm{SAT}}^{\kappa}\right)$. For the other direction let $f \in B\left(\Gamma_{\mathrm{SAT}}^{\kappa}\right)$. By definition of a bounded base we have $\mid$ domain $(f) \mid \leq \operatorname{ar}\left(\Gamma_{\mathrm{SAT}}^{\kappa}\right)=\kappa$ and $\operatorname{ar}(f) \leq$ \#tuples $\left(\Gamma_{\text {SAT }}^{\kappa}\right)=2^{\kappa}-1$. Now assume towards contradiction that $f$ is not a subfunction of a projection. Write $\kappa^{\prime} \leq \kappa$ for $\mid$ domain $(f) \mid$ and let $u_{1}, \ldots, u_{\kappa^{\prime}}$ be the tuples in domain $(f)$. Finally, for $i=1, \ldots, \kappa^{\prime}$ let $\ell_{i}$ 
be $x_{i}$ if $f\left(u_{i}\right)$ is 0 , and $\overline{x_{i}}$ if $f\left(u_{i}\right)$ is 1 , and let $R_{f}$ be the $\kappa^{\prime}$-ary relation $R_{f}$ defined by the clause $\left(\ell_{1} \vee \cdots \vee \ell_{\mathcal{K}^{\prime}}\right)$ Now since $f_{i}$ is not a subfunction of a projection and $u_{1}, \ldots, u_{\mathcal{K}^{\prime}}$ enumerate domain $(f)$, the tuple $\left(f\left(u_{1}\right), \ldots, f\left(u_{\mathcal{K}^{\prime}}\right)\right)$ is different from the tuple $\left(u_{1}[i], \ldots, u_{\mathcal{K}^{\prime}}[i]\right)$ for all $i=1, \ldots, \operatorname{ar}(f)$. Moreover, by construction of $R_{f},\left(f\left(u_{1}\right), \ldots, f\left(u_{\kappa^{\prime}}\right)\right)$ is not in $R_{f}$. Since $R_{f}$ is defined by a clause it contains all tuples but one, and it follows that it contains the tuple $\left(u_{1}[i], \ldots, u_{\kappa^{\prime}}[i]\right)$ for all $i=1, \ldots, \operatorname{ar}(f)$, but not the tuple $\left(f\left(u_{1}\right), \ldots, f\left(u_{\kappa^{\prime}}\right)\right)$. Hence $R_{f}$ is not preserved by $f$. On the other hand we have $R_{f}\left(x_{1}, \ldots, x_{\kappa^{\prime}}\right) \equiv R\left(x_{1}, \ldots, x_{\kappa^{\prime}}, x_{\kappa^{\prime}}, \ldots, x_{\kappa^{\prime}}\right)$ for an appropriately chosen $R \in \Gamma_{\mathrm{SAT}}^{\kappa}$, hence $f \in B\left(\Gamma_{\mathrm{SAT}}^{\kappa}\right) \subseteq \operatorname{pPol}\left(\Gamma_{\mathrm{SAT}}^{\kappa}\right)$ is a polymorphism of $R_{f}$, a contradiction.

Again, using bounded bases makes the characterisation much simpler, since $\operatorname{pPol}\left(\Gamma_{\mathrm{SAT}}^{\kappa}\right)$ includes functions with $\mid$ domain $(f) \mid=\lambda>\kappa$ which are not partial projections, as made clear in the following theorem. For a ground set $V$, a hypergraph $\mathcal{H} \subseteq 2^{V}$ is a set system over $V$, and a set $S \subseteq V$ is a hitting set of $\mathcal{H}$ if for every $E \in \mathcal{H}$ we have $E \cap S \neq \varnothing$.

Theorem 5.24. Let $f$ be an n-ary partial function that is not a partial projection, and let domain $(f)=\left\{u_{1}, \ldots, u_{k}\right\}$. Let $V=\left\{v_{1}, \ldots, v_{k}\right\}$, and define $\mathcal{H}=\left\{E_{1}, \ldots, E_{n}\right\} \subseteq 2^{V}$ by: $v_{i} \in E_{j}$ if and only if $u_{i}[j] \neq f\left(u_{i}\right)$. Then $f \in \operatorname{pPol}\left(\Gamma_{\mathrm{SAT}}^{\kappa}\right)$ if and only if every hitting set of $\mathcal{H}$ has size at least $\kappa+1$.

Proof. Observe that $\varnothing \notin \mathcal{H}$, since $f$ is not a partial projection. On the one hand, assume that $\mathcal{H}$ has a hitting set $S$ of size $\kappa$ or less. Consider the subfunction $f^{\prime}$ of $f$ with domain $\left(f^{\prime}\right)=\left\{u_{i} \mid v_{i} \in S\right\}$. By construction, $f^{\prime}$ is not a partial projection; thus $f^{\prime}$ has a contraction $g$ defined on at most $\kappa$ tuples and arity at most $2^{\kappa}-1$. By Theorem 5.23, $g$ is not a partial polymorphism of $\kappa$-SAT, hence neither is $f$. On the other hand, assume that all hitting sets of $\mathcal{H}$ have size at least $\kappa+1$. Let $B$ be the bounded base for $\kappa$-SAT; by Theorem 5.15. we must show $f \in C l_{\kappa, n}(B)$. Let $f^{\prime}$ be a restriction of $f$ on at most $\kappa$ tuples, and let $S=\left\{v_{i} \mid u_{i} \in \operatorname{domain}\left(f^{\prime}\right)\right\}$. Then $S$ is not a hitting set of $\mathcal{H}$, hence for some $E_{j} \in \mathcal{H}$ we have $S \cap E_{j}=\varnothing$, hence $f^{\prime}$ is a subfunction of $\pi_{j}^{n}$. Now we have a few simple cases. If $n \leq 2^{\kappa}-1$, then $f^{\prime} \in B$; if $n>2^{\kappa}-1$ but $f^{\prime}$ has a contraction of arity at most $2^{\kappa}-1$, then $f^{\prime}$ is a stretching of a function in $B$; otherwise $f^{\prime} \in \mathcal{U}_{n}^{2^{\kappa}-1}$ by definition of $\mathcal{U}_{n}^{m}$. In each case, we see that we can construct a $\kappa$-covering of $f$ from $B\left(\Gamma_{\mathrm{SAT}}^{\kappa}\right)$, hence $f \in C l_{\kappa, n}(B)$ and we are done.

\subsection{Summary and Conclusions}

We have proposed the new notion of a bounded base which, together with a novel closure operator, allows one to characterise the partial polymorphisms of a finite constraint language from a finite set of partial functions. This notion gives simple characterisations of the partial polymorphisms of natural and well-studied languages. 



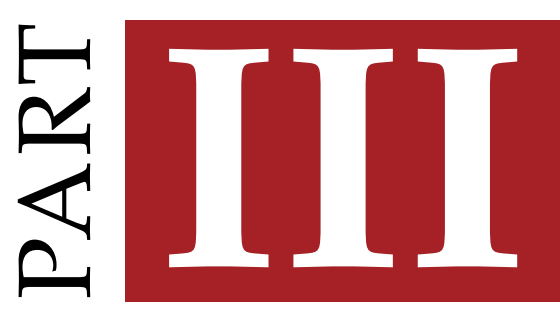

\section{Complexity of Constraint Satisfaction Problems}





\section{Size-Preserving}

Reductions and the Exponential-Time Hypothesis

- Comb-me quickly; don't put me so much pomatum. What news tell me? all hairs dresser are newsmonger.

- Sir, I have no heared any thing.

English as she is spoke

So far we have mainly been concerned with weak bases and properties of strong partial clones. In this part of the thesis we shift the focus toward applications within computer science, and use partial clone theory to study the computational complexity of constraint satisfaction problems. In this chapter we introduce the computational problems that will be studied in the forthcoming chapters, and introduce the concept of size-preserving reductions, which will allow us to study the complexity of these problems in a more finegrained manner than possible with ordinary polynomial-time reductions.

\subsection{Problems Under Consideration}

The weighted maximum ones problem over $S$ (W-MAX-ONES $(S)$ ) is an optimisation version of SAT $(S)$ where we for an instance on variables $\left\{x_{1}, \ldots, x_{n}\right\}$ and weights $w_{i} \in \mathbb{Q}_{\geq 0}$ want to find a solution $h$ for which $\sum_{i=1}^{n} w_{i} h\left(x_{i}\right)$ is maximal. We can formulate $\mathrm{W}-\operatorname{MAX}-\operatorname{ONES}(S)$ as a decision problem in NP by supplying a natural number $k$ and ask whether there is a solution $h$ such that $\sum_{i=1}^{n} w_{i} h\left(x_{i}\right) \geq k$. The unweighted maximum ones problem 
(U-MAX-ONES $(S))$ is the $\mathrm{W}-\mathrm{MAX}-\mathrm{OnES}(S)$ problem where all weights have the value 1 . If it in a particular context does not matter whether the problem is weighted or not we typically write MAX-ONES.

A finite-valued cost function on $\mathbb{B}$ is a function $f: \mathbb{B}^{n} \rightarrow \mathbb{Q}_{\geq 0}$. The valued constraint satisfaction problem over a finite set of finite-valued cost functions $\Delta$ $(\operatorname{VCSP}(\Delta))$ is the problem of, given a set of variables $V$ and a set of weight $W$, minimising a sum of the form $\Sigma_{i=1}^{q} f_{i}\left(\mathbf{x}_{\mathbf{i}}\right)$, where $f_{i} \in \Delta$ and $\mathbf{x}_{\mathbf{i}} \in V^{\operatorname{ar}(f)}$. Similar to the case of MAX-ONES we can treat VCSP as a decision problem by supplying a number $k$ and ask whether the sum is smaller than or equal to $k$.

Note that the instances of the problems $\operatorname{SAT}(\cdot), \operatorname{MAX}-\mathrm{ONES}(\cdot)$ and $\operatorname{VCSP}(\cdot)$ all share a similar structure, in the sense that they all can be seen as problems where the goal is to assign values from a fixed finite set to a collection of variables. We call problems of this kind variable problems and let $\operatorname{Var}(I)$ denote the set of variables of an instance $I$. In the context of this thesis, the term 'variable problem' is essentially treated as a shorthand for any of the problems defined in this section, but also for certain graph problems such as W-MAX-CUT and W-MAX INDEPENDENT SET, i.e., the problems of finding a cut or an independent set in a weighted graph of size larger than or equal to some $k \geq 0$. In these two cases we let $\operatorname{Var}(I)$ denote the number of vertices of an instance $I$.

\subsection{Subexponential Time, Size-Preserving Reductions and the Exponential-Time Hypothesis}

If $A$ is a computational problem we let $I(A)$ be the set of problem instances and $\|I\|$ be the size of any $I \in I(A)$, i.e. the number of bits required to represent $I$. A problem solvable in $O\left(2^{c|\operatorname{Var}(I)|}\right)$ time for all $c>0$ is a subexponential problem, and we let SE denote the class of all variable problems solvable in subexponential time. Naturally, SE can be defined using other complexity parameters than $|\operatorname{Var}(I)|$ [IP01].

The exponential-time hypothesis (ETH) states that 3-SAT $\notin$ SE [IPZ01]. We remind the reader that the ETH can be based on different size parameters (such as the number of variables or the number of clauses) and that these different definitions often coincide [IP01]. Note that even if $A_{1}$ and $A_{2}$ are two variable problems in NP such that $A_{2} \in \mathrm{SE}$ and $A_{1}$ is polynomial-time many-one reducible to $A_{2}$, then this does not automatically imply that $A_{1} \in$ SE. To compare problems with respect to subexponential time complexity we therefore need a more restriction notion of a polynomial-time reduction.

Definition 6.1. Let $A_{1}$ and $A_{2}$ be variable problems in NP. The function $f$ from $I\left(A_{1}\right)$ to $I\left(A_{2}\right)$ is a many-one linear variable reduction (LV-reduction) with parameter $C \geq 0$ if:

1. $I$ is a yes-instance of $A_{1}$ if and only if $f(I)$ is a yes-instance of $A_{2}$,

2. $|\operatorname{Var}(f(I))|=C \cdot|\operatorname{Var}(I)|+O(1)$, and 
3. $f(I)$ can be computed in $O($ poly $(\|I\|))$ time.

Note that LV-reductions can be seen as a restricted form of SERFreductions [IP01]. The term CV-reduction is used to denote $\mathrm{LV}$-reductions with parameter 1 , and we write $A_{1} \leq \mathrm{CV} A_{2}$ to denote that the problem $A_{1}$ has a CV-reduction to $A_{2}$. We have the following two obvious theorems concerning LV-reductions and CV-reductions.

Theorem 6.2. Let $A_{1}$ and $A_{2}$ two variable problems in NP such that $A_{1}$ is solvable in $O\left(\operatorname{poly}(\|I\|) \cdot c^{|\operatorname{Var}(I)|}\right)$ time. If there exists an $L V$-reduction from $A_{2}$ to $A_{1}$ with parameter $C$, then $A_{2}$ can be solved in $O\left(\operatorname{poly}(\|I\|) \cdot d^{|\operatorname{Var}(I)|}\right)$ time, where $d=c^{C}$.

In particular, if $A_{1} \in \mathrm{SE}$ and $A_{2}$ is $\mathrm{LV}$-reducible to $A_{1}$, then $A_{2} \in \mathrm{SE}$, too. Hence, LV-reductions are a useful tool for comparing problems with respect to subexponential complexity. CV-reductions, on the other hand, are useful when comparing the complexity of variable problems up to $O\left(c^{|\operatorname{Var}(I)|}\right)$ time complexity.

Theorem 6.3. Let $A_{1}$ and $A_{2}$ be two variable problems in NP such that $A_{1}$ is solvable in $O\left(\operatorname{poly}(\|I\|) \cdot c^{|\operatorname{Var}(I)|}\right)$ time. If there exists a $C V$-reduction from $A_{2}$ to $A_{1}$, then $A_{2}$ can be solved in $O\left(\operatorname{poly}(\|I\|) \cdot c^{|\operatorname{Var}(I)|}\right)$ time.

If $A_{1}$ and $A_{2}$ are two NP-complete variable problems we say that $A_{1}$ is at least as easy as (or not harder than) $A_{2}$ if $A_{1}$ is solvable in $O\left(c^{|\operatorname{Var}(I)|}\right)$ time whenever $A_{1}$ is solvable in $O\left(c^{|\operatorname{Var}(I)|}\right)$ time. Sometimes, we simply say that $A_{1}$ is easier than $A_{2}$ if this condition holds. By definition, if $A_{1} \leq \mathrm{CV} A_{2}$ then $A_{1}$ is not harder than $A_{2}$ but the converse is not true in general. 



\section{The Boolean Satisfiability}

Problem

- You have a bad tooth; will you pull out this tooth?

- I can't to decide me it, that make me many great deal pain.

- Your tooth is absolutely roted; if you leave it; shall spoil the others.

English as she is spoke

In this chapter we study the complexity of the SAT $(\cdot)$ problem using the size-preserving reductions defined in the previous chapter. We first prove that partial polymorphisms can be used to compare the worst-case running times of $\operatorname{SAT}(\cdot)$ problems. With the help of this algebraic approach we are then find a concrete relation $R_{1 / 3}^{\neq \neq \neq}$such that $\operatorname{SAT}\left(\left\{R_{1 / 3}^{\neq \neq \neq}\right\}\right)$in a precise way can be referred to as the computationally easiest NP-complete SAT $(\cdot)$ problem. Using this result we are then able to prove that if there exists one NPcomplete $\operatorname{SAT}(S)$ problem such that $\operatorname{SAT}(S) \in \mathrm{SE}$, then all NP-complete $\mathrm{SAT}(\cdot)$ problems are solvable in subexponential time.

\subsection{Introduction}

Let $S$ be a constraint language such that SAT $(S)$ is NP-complete. What is the time complexity of solving $\operatorname{SAT}(S)$ ? Is $\operatorname{SAT}(S)$ solvable in subexponential time or does there exist a concrete $c \in \mathbb{R}$ such that $\operatorname{SAT}(S)$ is solvable in 
$O\left(c^{|\operatorname{Var}(I)|}\right)$ time, but not strictly faster than this? Ultimately, one would like to have a 'table' that for each NP-complete SAT $(S)$ problem not solvable in subexponential time, contains a number $c$ such that $\operatorname{SAT}(S)$ can be solved in $\Theta\left(c^{|\operatorname{Var}(I)|}\right)$ time but not faster. It seems that we are very far from this goal, unfortunately. Let us imagine a weaker qualitative approach: construct a table that for every two problems $\operatorname{SAT}(S)$ and $\operatorname{SAT}\left(S^{\prime}\right)$ tells us whether $\operatorname{SAT}(S)$ and $\operatorname{SAT}\left(S^{\prime}\right)$ can be solved equally fast, whether $\operatorname{SAT}(S)$ can be solved strictly faster than $\operatorname{SAT}\left(S^{\prime}\right)$, or vice versa (assuming $P \neq N P$ ). That is, we have access to the underlying total order on running times but we cannot say anything about the exact figures. Not surprisingly, we are far from this goal, too. However, as will be made clear in this chapter, the lattice of strong partial clones is a surprisingly useful approximation of such a table, and can be used to draw several non-trivial conclusions.

\section{The Easiest NP-complete SAT (.) Problem}

We begin in Section 7.3 by proving that partial polymorphisms can be used for comparing NP-complete SAT $(\cdot)$ problems with respect to $\mathrm{CV}$-reductions. More precisely, we prove that $\mathrm{SAT}(S) \leq{ }^{\mathrm{CV}} \operatorname{SAT}\left(S^{\prime}\right)$ if $\mathrm{pPol}\left(S^{\prime}\right) \subseteq \operatorname{pPol}(S)$. This result can be seen as an analogue of Theorem 2.13 in the context of partial polymorphisms instead of total polymorphisms. While this result shows that partial polymorphisms are indeed useful when comparing NP-complete problems with respect to worst-case time complexity, recall from Section 2.4.1 in Chapter 2 that the lattice of strong partial clones is of uncountably infinite cardinality even for the Boolean domain. Hence, a priori, there could exist an uncountable number of possible cases of the worst-case time complexity of $\operatorname{SAT}(\cdot)$. Despite this, we in Section 7.4 show, by utilising the work from Chapter 3 concerning weak bases, that there is a certain structure in the lattice strong partial clones that enable us to find a constraint language $S$ such that $\operatorname{SAT}(S)$ is the easiest NP-complete $\mathrm{SAT}(S)$ problem. The easiest NPcomplete $\mathrm{SAT}(S)$ problem is surprisingly simple: $S$ consists of a single 6-ary relation $R_{1 / 3}^{f \neq \neq}$ which contains the three tuples $(1,0,0,0,1,1),(0,1,0,1,0,1)$, and $(0,0,1,1,1,0)$.

Obviously the first question that any astute reader would ask is exactly how easy $\operatorname{SAT}\left(\left\{R_{1 / 3}^{\neq \neq \neq}\right\}\right)$is compared to other $\operatorname{SAT}(\cdot)$ problems. We answer this question in Section 7.5 and relate the complexity of $\operatorname{SAT}\left(\left\{R_{1 / 3}^{\neq \neq \neq}\right\}\right)$to 1-IN-3-SAT, and prove that $\operatorname{SAT}\left(\left\{R_{1 / 3}^{\neq \neq \neq}\right\}\right)$is solvable in time $O\left(2^{(c+\varepsilon) n}\right)$ for all $\varepsilon>0$ if and only if 1-IN-3-SAT is solvable in time $O\left(2^{(2 c+\varepsilon) n}\right)$ for all $\varepsilon>0$. By 1-IN-3-SAT we mean SAT $(S)$ where $S$ contains all ternary relations corresponding to exactly one of three literals being assigned to true, not to be confused with MONOTONE 1-IN-3-SAT, which we in this thesis typically denote by $\operatorname{SAT}\left(\left\{R_{1 / 3}\right\}\right)$. Similar results are also proven for other languages that like $R_{1 / 3}^{\neq \neq \neq}$contain a sufficient number of complementary arguments.

We note that there has been an interest in identifying extremely easy NPcomplete problems before. For instance, van Rooij et al. [RKNB13] have shown that the PARTITION INTO TRIANGLES problem restricted to graphs 
of maximum degree four can be solved in $O\left(1.02445^{n}\right)$ time. They argue that practical algorithms may arise from this kind of studies, and the very same observation has been made by, for instance, Woeginger [Woe03]. It is important to note that our results give much more information than just the mere fact that $\operatorname{SAT}\left(\left\{R_{1 / 3}^{\neq \neq \neq}\right\}\right)$is easy to solve; they also tell us how this problem is related to all other problems within the large and diverse class of $\mathrm{SAT}(S)$ problems. This is one of the major advantages in using the clonetheoretical approach when studying this kind of questions. Another reason to study such problems is that they, in some sense, are close to the borderline between problems in $\mathrm{P}$ and NP-complete problems (here we tacitly assume that $\mathrm{P} \neq \mathrm{NP}$ ). The structure of this borderline has been studied with many different aims and methods; two well-known examples are the articles by Ladner [Lad75] and Schöning [Sch83].

Having determined the easiest SAT $(\cdot)$ problem, it is natural to investigate other properties of the lattice of strong partial clones. We do this in Section 7.6 and focus on two aspects. First, we provide a partial classification of all Boolean constraint languages below MONOTONE 1-IN-3-SAT and among other prove that the relations $R_{1 / 3}^{\neq \neq}=\{(1,0,0,0,1),(0,1,0,1,0),(0,0,1,1,1)\}$ and $R_{1 / 3}^{\neq}=\{(1,0,0,0),(0,1,0,1),(0,0,1,1)\}$ reside in this structure. We conjecture that the strong partial clones corresponding to these languages cover each other in the sense that there are no languages of intermediate complexity in between. If this is true then $\operatorname{SAT}\left(\left\{R_{1 / 3}^{\neq \neq}\right\}\right)$and $\operatorname{SAT}\left(\left\{R_{1 / 3}^{\neq}\right\}\right)$can be regarded as the second easiest and third easiest $\mathrm{SAT}(\cdot)$ problems, respectively. Combined with the results from Section 7.5 this also shows that all Boolean constraint languages below 1-IN-3-SAT are in the worst case solvable in time $O\left(2^{(2 c+\varepsilon) n}\right)$ for all $\varepsilon>0$ if the easiest problem $\operatorname{SAT}\left(\left\{R_{1 / 3}^{\neq \neq \neq}\right\}\right)$is solvable in time $O\left(2^{(c+\varepsilon) n}\right)$ for all $\varepsilon>0$. Second, we show that both MONOTONE 1 -IN- $\kappa$-SAT and $\kappa$-SAT correspond to different strong partial clones for every $\kappa$ and also that the strong partial clones corresponding to MONOTONE $1-\mathrm{IN}-(\kappa+1)$-SAT and $\kappa$-SAT are incomparable. These proofs do not require any particular complexity theoretical assumptions and may be interesting to compare with existing work on the complexity of $\kappa$-SAT [IP01].

\section{Subexponential Complexity and LV-reductions}

The second contribution of this chapter (Section 7.8) is devoted to relating the complexity of SAT $(\cdot)$ problems to the ETH [IPZ01]. The ETH has recently gained popularity when studying the computational complexity of combinatorial problems, cf. the survey by Lokshtanov et al. [LMS11].

To study the implications of the ETH for the SAT $(\cdot)$ problem we utilise LV-reductions instead of CV-reductions, since the former results in more powerful reductions but still preserve subexponential complexity. We let the results in the previous sections guide us by exploiting the $\operatorname{SAT}\left(\left\{R_{1 / 3}^{\neq \neq \neq}\right\}\right)$ problem. This problem is CV-reducible (and thus trivially LV-reducible) to any NP-complete SAT $(S)$, but the converse question of which $S A T(S)$ problems are $\operatorname{LV}$-reducible to $\operatorname{SAT}\left(\left\{R_{1 / 3}^{\neq \neq \neq}\right\}\right)$is more challenging. By utilising spar- 
sification [IP01] IPZ01], we can attack the more general problem of identifying degree bounded SAT $(S)$-DEG- $B$ problems that are subexponential if and only if 3-SAT is subexponential. Here SAT(S)-DEG- $B$ denotes the SAT $(S)$ problem restricted to instances where each variable occurs in at most $B$ constraints. We do this in Section 7.8.3 and prove that the exponential-time hypothesis holds if and only if either of $\operatorname{SAT}\left(\left\{R_{1 / 3}^{\neq \neq \neq}\right\}\right)$-DEG-2, $\operatorname{SAT}\left(\left\{R_{1 / 3}^{\neq \neq}\right\}\right)$DEG-2 or $\operatorname{SAT}\left(\left\{R_{1 / 3}^{\neq}\right\}\right)$-DEG-2 cannot be solved in subexponential time. An important ingredient in the proof is the result (proven in Section 7.7) that $\operatorname{SAT}\left(\left\{R_{1 / 3}^{\neq \neq \neq}\right\}\right)$-DEG-2 is NP-complete. This also holds for $\operatorname{SAT}\left(\left\{R_{1 / 3}^{\neq \neq}\right\}\right)$DEG-2 and $\operatorname{SAT}\left(\left\{R_{1 / 3}^{\neq}\right\}\right)$-DEG-2. We prove this by using results by Dalmau and Ford [DF03] combined with the fact that $R_{1 / 3}^{\neq \neq \neq}, R_{1 / 3}^{\neq \neq}$and $R_{1 / 3}^{\neq}$are not $\Delta$ matroid relations. This should be contrasted with MONOTONE 1-IN-3-SAT or the unrestricted SAT problem, which are in $P$ under the same restriction. We conclude that $\operatorname{SAT}\left(\left\{R_{1 / 3}^{\neq \neq \neq}\right\}\right)$-DEG-2, $\operatorname{SAT}\left(\left\{R_{1 / 3}^{\neq \neq}\right\}\right)$-DEG-2 and $\operatorname{SAT}\left(\left\{R_{1 / 3}^{\neq}\right\}\right)$DEG-2 are all good examples of problems with extremely simple structures but which remain NP-complete.

Combining these results we show the following consequence: if ETH does not hold, then SAT(S)-DEG- $B$ is subexponential for every $B$ whenever $S$ is finite. Thus, under LV-reductions, all SAT $(S)$ problems and many SAT $(S)$-DEG- $B$ problems are equally hard. Impagliazzo et al. [IPZ01] prove that many NP-complete problems in the complexity class SNP (which contains the $\operatorname{SAT}(\cdot)$ problem) are subexponential if and only if $\kappa$-SAT is subexponential. Hence, we strengthen this result when restricted to SAT $(\cdot)$ problems. In the process, we also prove a stronger version of Impagliazzo et al.'s [IPZ01] sparsification lemma for $\kappa$-SAT; namely that all finite Boolean constraint languages $S$ and $S^{\prime}$ such that both $\operatorname{SAT}(S)$ and $\operatorname{SAT}\left(S^{\prime}\right)$ are NPcomplete can be sparsified into each other. This can be contrasted with Santhanam's and Srinivasan's [SS12] negative result, which states that the same does not hold for the unrestricted SAT problem and, consequently, not for all infinite Boolean constraint languages.

\subsection{Preliminaries}

Since we in this chapter are interested in the worst-case time complexity of $\operatorname{SAT}(\cdot)$ problems we introduce some simplifying notation: let $\mathcal{H}$ denote the set of all finite Boolean constraint languages $S$ such that $\operatorname{SAT}(S)$ is NPcomplete, and define the function $\mathrm{T}: \mathcal{H} \rightarrow \mathbb{R}^{+}$such that

$$
\mathrm{T}(S)=\inf \left\{c \mid \operatorname{SAT}(S) \text { can be solved in time } O\left(2^{c \cdot|\operatorname{Var}(I)|}\right)\right\} .
$$

Note that if $\mathrm{T}(S)=0$ then $\mathrm{SAT}(S) \in \mathrm{SE}$. Hence, the ETH can be restated as the conjecture that $T\left(\Gamma_{S A T}^{3}\right)>0$. We can now define the concept of 'easier than' from Section 6.2 in the previous chapter using the function T.

Definition 7.1. Let $S, S^{\prime} \in \mathcal{H}$. If $\mathrm{T}(S) \leq \mathrm{T}\left(S^{\prime}\right)$ then we say that $\mathrm{SAT}(S)$ is easier than $\mathrm{SAT}\left(S^{\prime}\right)$, and if $\mathrm{T}(S)<\mathrm{T}\left(S^{\prime}\right)$ then we say that $\mathrm{SAT}(S)$ is strictly easier than $\operatorname{SAT}\left(S^{\prime}\right)$. 
Note that the second case can only occur if $\operatorname{SAT}\left(S^{\prime}\right)$ is not solvable in subexponential time.

We conclude this section with a few words about bounded degree instances. Let $S$ be a constraint language and $\phi$ an instance of SAT $(S)$. If $x$ occurs in $B$ constraints in $\phi$, then we say that the degree of $x$ is $B$. We let SAT(S)-DEG- $B$ denote the $\operatorname{SAT}(S)$ problem where each variable in the input is restricted to have degree at most $B$. Similarly, we let SAT $(S)$-OCC- $B$ denote the SAT $(S)$ problem where in each instance each variable can occur at most $B$ times. The difference between the two notions is that in the latter case the total number of occurrences of variables, also within constraints, are counted, while in the former only the degrees of variables are considered. For example, if $\phi=R_{1 / 3}(x, y, y) \wedge R_{1 / 3}(x, z, w)$, then $x$ has degree 2 and $y, z, w$ degree 1 , but $x$ and $y$ have the same number of occurrences. Obviously if SAT $(S)$-DEG- $B$ is in $\mathrm{P}$ then SAT $(S)$-OCC-B is also in P, and if SAT $(S)$-OCC-B is NP-complete then SAT( $S)$-DEG- $B$ is NP-complete. These restrictions are of particular interest, since, for all constraint languages $S$ such that $\operatorname{SAT}(S)$ is NP-complete, $\mathrm{SAT}(S)$-OCC- $B$ is NP-complete for some $B$.

Theorem 7.2 (Jonsson et al. [JKK09]). If $S \in \mathcal{H}$, then there exists an integer $B$ such that SAT(S)-OCC-B is NP-complete.

Hence, the same also holds for SAT $(S)$-DEG- $B$.

\subsection{Partial Polymorphisms and CV-Reductions}

In this section we prove that the partial polymorphisms of a finite constraint language $S$ determines the complexity of SAT $(S)$ up to $O\left(c^{|\operatorname{Var}(I)|}\right)$ time complexity. The proof is fairly straightforward due to the Galois connection in Theorem 2.21.

Theorem 7.3. Let $S$ and $S^{\prime}$ be finite constraint languages over a finite domain $D$. If $\mathrm{pPol}\left(S^{\prime}\right) \subseteq \operatorname{pPol}(S)$ then $\operatorname{CSP}(S)$ is $C V$-reducible to $\operatorname{CSP}\left(S^{\prime}\right)$.

Proof. Assume that $\mathrm{pPol}\left(S^{\prime}\right) \subseteq \operatorname{pPol}(S)$. According to the Galois connection in Theorem 2.21 this implies that $S \subseteq\left\langle S^{\prime}\right\rangle_{\nexists}$, or, equivalently, that every $R \in S$ can be expressed as a q.f.p.p. definition over $S^{\prime}$. Given an instance $\phi$ of $\operatorname{CSP}(S)$ over a set of variables $V$, we can transform $\phi^{\prime}$ (in $O($ poly $(|V|))$ time) into an equivalent instance $\phi^{\prime}$ of $\operatorname{CSP}\left(S^{\prime}\right)$ containing at most $|V|$ variables as follows. Since $S$ is fixed and finite, we can assume that the q.f.p.p. implementation of each relation in $S$ over $S^{\prime}$ has been precomputed and stored in a table (of fixed constant size). Every constraint $R\left(x_{1}, \ldots, x_{\mathcal{K}}\right)$ in $\phi$ can be represented as

$$
R_{1}\left(\mathbf{x}_{1}\right) \wedge \ldots \wedge R_{l}\left(\mathbf{x}_{\mathbf{l}}\right)
$$

where $R_{1}, \ldots, R_{l} \in S^{\prime} \cup\{\mathrm{Eq}\}$ and each $\mathbf{x}_{\mathbf{i}}$ is a tuple over variables over $\left\{x_{1}, \ldots, x_{\kappa}\right\}$. Replace the constraint $R\left(x_{1}, \ldots, x_{\kappa}\right)$ with the constraints $R_{1}\left(\mathbf{x}_{\mathbf{1}}\right) \wedge \ldots \wedge R_{l}\left(\mathbf{x}_{\mathbf{1}}\right)$. If we repeat the same reduction for every constraint 
in $\phi$, it results in an equivalent instance of $\operatorname{CSP}\left(S^{\prime} \cup\{\mathrm{Eq}\}\right)$ with at most $|V|$ variables. For each equality constraint $\operatorname{Eq}\left(x_{i}, x_{j}\right)$, we replace all occurrences of $x_{i}$ with $x_{j}$, and remove the equality constraint. The resulting instance $I^{\prime}$ is an equivalent instance of $\operatorname{CSP}\left(S^{\prime}\right)$ with at most $|V|$ variables. Finally, since $S$ is finite, there cannot be more than $|V|^{p} \cdot|S|$ constraints in $I$, where $p$ is the highest arity of a relation in $S$. It follows that computing $I^{\prime}$ from $I$ can be done in $O\left(|V|^{p}\right)$ time, which concludes the proof.

Recall that if $D$ is a finite domain, $R$ a $\kappa$-ary relation over $D$, and $a_{1}, \ldots, a_{k} \in D$, then $R^{a_{1} \ldots a_{k}}$ denotes the relation $R^{a_{1} \ldots a_{k}}=R \times\left\{\left(a_{1}, \ldots, a_{k}\right)\right\}$. It should not come as a surprise that if the constants $a_{1}, \ldots, a_{k}$ can be q.f.p.p. defined over $R$ then $\operatorname{CSP}(\{R\})$ and $\operatorname{CSP}\left(\left\{R^{a_{1} \ldots a_{k}}\right\}\right)$ have the same worst-case time complexity.

Theorem 7.4. Let $R$ be a relation over a finite domain $D$ and let $a_{1}, \ldots, a_{k} \in$ D. If $\left\{\left(a_{1}, \ldots, a_{k}\right)\right\} \in\langle\{R\}\rangle_{\exists}$ then $\operatorname{CSP}\left(\left\{R^{a_{1} \ldots a_{k}}\right\}\right) \leq \mathrm{CV} \operatorname{CSP}(\{R\})$ and $\operatorname{CSP}(\{R\}) \leq{ }^{\mathrm{CV}} \operatorname{CSP}\left(\left\{R^{a_{1} \ldots a_{k}}\right\}\right)$.

Proof. It is not difficult to see that $\left\{\left(a_{1}, \ldots, a_{k}\right)\right\} \in\left\langle\left\{R^{a_{1} \ldots a_{k}}\right\}\right\rangle_{\nexists}$ since $\left\{\left(a_{1}, \ldots, a_{k}\right)\right\} \in\langle\{R\}\rangle_{\nexists \text { F }}$. From this observation it follows that

$$
\begin{array}{r}
R^{a_{1} \ldots a_{k}}\left(x_{1}, \ldots, x_{\operatorname{ar}(R)}, x_{\operatorname{ar}(R)+1}, \ldots, x_{\operatorname{ar}(R)+k}\right) \equiv \\
R\left(x_{1}, \ldots, x_{\operatorname{ar}(R)}\right) \wedge \\
\left\{\left(a_{1}, \ldots, a_{k}\right)\right\}\left(x_{\operatorname{ar}(R)+1}, \ldots, x_{\operatorname{ar}(R)+k}\right),
\end{array}
$$

i.e., $R^{a_{1} \ldots a_{k}} \in\left\langle\left\{R,\left\{\left(a_{1}, \ldots, a_{k}\right)\right\}\right\}\right\rangle_{\nexists}=\langle\{R\}\rangle_{\nexists \exists}$. Hence, $\operatorname{pPol}\left(\left\{R^{a_{1} \ldots a_{k}}\right\}\right) \supseteq$ $\operatorname{pPol}(\{R\})$, and we can directly apply Theorem 7.3 to deduce that $\operatorname{CSP}\left(\left\{R^{a_{1} \cdots a_{k}}\right\}\right) \leq \mathrm{CV} \operatorname{CSP}(\{R\})$. The second direction, to prove that $\operatorname{CSP}(\{R\}) \leq \mathrm{CV} \operatorname{CSP}\left(\left\{R^{a_{1} \ldots a_{k}}\right\}\right)$, requires a bit more care, since in general, $\operatorname{pPol}(\{R\}) \nsupseteq \operatorname{pPol}\left(\left\{R^{a_{1} \ldots a_{k}}\right\}\right)$. Let $I=(V, C)$ be an instance of $\operatorname{CSP}(\{R\})$. We provide a CV-reduction from $\operatorname{CSP}(\{R\})$ to $\operatorname{CSP}\left(\left\{R^{a_{1} \ldots a_{k}},\left\{\left(a_{1}, \ldots, a_{k}\right)\right\}\right\}\right)$. Let $y_{1}, \ldots, y_{k}$ be $k$ fresh variables and introduce the constraint $\left\{\left(a_{1}, \ldots, a_{k}\right)\right\}\left(y_{1}, \ldots y_{k}\right)$. For each constraint $R\left(x_{1}, \ldots, x_{\operatorname{ar}(R)}\right) \in C$ then replace it by the constraint $R^{a_{1} \ldots a_{k}}\left(x_{1}, \ldots, x_{\operatorname{ar}(R)}, y_{1}, \ldots, y_{k}\right)$. Again, it is easy to see that the resulting instance is satisfiable if and only if $I$ is satisfiable.

The proof of Theorem 7.4 while not complicated, illustrates two useful techniques for obtaining a CV-reduction from $\operatorname{CSP}(S)$ to $\operatorname{CSP}\left(S^{\prime}\right)$. The first technique is to directly apply Theorem 7.3 by proving that each relation in $S$ has a q.f.p.p. definition over $S^{\prime}$. When this is not possible, the second technique is to circumvent the limitations of q.f.p.p. definitions by introducing a constant number of variables that are shared between constraints. 


\subsection{The Easiest NP-complete SAT $(S)$ Problem}

In this section we will use the theory and results presented in previous chapters in this thesis to determine the easiest NP-complete SAT $(S)$ problem. Recall that by easiest we mean that if any NP-complete $\mathrm{SAT}(S)$ problem can be solved in $O\left(c^{|V|}\right)$ time, then the easiest problem can be solved in $O\left(c^{|V|}\right)$ time, too. By combining Theorem 3.3 and Theorem 7.3 we immediately obtain the following characterisation of weak bases.

Theorem 7.5. Let $\operatorname{Inv}(\mathrm{C})$ be a co-clone of finite order over a finite domain. Let $S_{w}$ be a finite weak base of $\operatorname{Inv}(\mathrm{C})$. Then $\operatorname{CSP}\left(S_{w}\right) \leq \mathrm{CV} \operatorname{CSP}(S)$ for any finite base $S$ of $\operatorname{Inv}(\mathrm{C})$.

Now note that, assuming $\mathrm{P} \neq \mathrm{NP}$, if $\operatorname{CSP}(S)$ is NP-complete and solvable in $O\left(c^{|V|}\right)$ time, then $\operatorname{CSP}\left(S_{w}\right)$ is solvable in $O\left(c^{|V|}\right)$ time, too. In other words the weak base of a co-clone results in the constraint satisfaction problem over the co-clone with the lowest worst-case time complexity. Now, recall that $\operatorname{SAT}(S)$ is NP-complete if and only if $\langle S\rangle=\mathrm{IN}_{2}$ or $\langle S\rangle=\mathrm{BR}$. This suggests that there are only two candidate languages resulting in the easiest NP-complete SAT $(\cdot)$ problem: the weak base of BR and the weak base of $\mathrm{IN}_{2}$. Hence, let $R_{1 / 3}^{\neq \neq \neq 1}$ denote the weak base of BR in Table 3.1 from Chapter 3. Recall from Section 2.3.2 in Chapter 2 that if $R$ is a $\kappa$-ary relation we by $R^{l \neq}$ denote the $(\kappa+l)$-ary relation defined as

$$
\begin{array}{r}
R^{l \neq}\left(x_{1}, \ldots, x_{\kappa}, x_{\kappa+1}, \ldots, x_{\kappa+l}\right) \equiv R\left(x_{1}, \ldots, x_{\kappa}\right) \wedge \\
\operatorname{Neq}\left(x_{1}, x_{\kappa+1}\right) \wedge \ldots \wedge \operatorname{Neq}\left(x_{\kappa}, x_{\kappa+l}\right),
\end{array}
$$

and that $R^{01}=R \times\{(0,1)\}$. In other words $R_{1 / 3}^{\neq \neq \neq 01}$ and $R_{1 / 3}^{\neq \neq \neq}$can be defined as

$$
R_{1 / 3}^{\neq \neq \neq 01}=\{(0,0,1,1,1,0,0,1),(0,1,0,1,0,1,0,1),(1,0,0,0,1,1,0,1)\} ，
$$

and

$$
R_{1 / 3}^{\neq \neq \neq}=\{(0,0,1,1,1,0),(0,1,0,1,0,1),(1,0,0,0,1,1)\} .
$$

Furthermore, let the relations $R_{1 / 3}^{\neq \neq}$and $R_{1 / 3}^{\neq}$be the two relations obtained from removing one respectively two complemented arguments from $R_{1 / 3}^{\neq \neq \neq}$. These two relations are related to the structure of the weak partial co-clones in the 'bottom of BR', which we expand upon in Section 7.6

Lemma 7.6. Let $S$ be a finite constraint language such that $\langle S\rangle=\mathrm{BR}$. Then $\operatorname{SAT}\left(\left\{R_{1 / 3}^{\neq \neq \neq}\right\}\right)$is easier than $\operatorname{SAT}(S)$.

Proof. From Theorem 7.5 it follows that $\operatorname{CSP}\left(\left\{R_{1 / 3}^{\neq \neq \neq 01}\right\}\right) \leq \mathrm{CV} \operatorname{CSP}(S)$ since $R_{1 / 3}^{\neq \neq \neq 01}$ is a weak base of BR. Let $R_{0,1}=\{(0,1)\}$ and note that $R_{0,1} \in$ $\left\langle\left\{R_{1 / 3}^{\neq \neq \neq}\right\}\right\rangle_{\nexists}$ since $R_{0,1}\left(x_{1}, x_{2}\right) \equiv R_{1 / 3}^{\neq \neq \neq}\left(x_{1}, x_{1}, x_{2}, x_{2}, x_{2}, x_{1}\right)$. Hence, by applying Theorem 7.4 it follows that $\operatorname{CSP}\left(\left\{R_{1 / 3}^{\neq \neq \neq}\right\}\right) \leq \operatorname{CV} \operatorname{CSP}\left(\left\{R_{1 / 3}^{\neq \neq \neq 01}\right\}\right)$, and that $\operatorname{CSP}\left(\left\{R_{1 / 3}^{\neq \neq \neq}\right\}\right)$is easier than $\operatorname{CSP}(S)$ since we assume that $\mathrm{P} \neq \mathrm{NP}$. 
Lemma 7.6 implies that we can choose to work with the relation $R_{1 / 3}^{\neq \neq \neq}$instead of the slightly more complicated weak base $R_{1 / 3}^{\neq \neq 01}$. We are now left with the co-clone $I_{2}$ and have to ensure that the weak base of $\mathrm{IN}_{2}$ does not result in a constraint satisfaction problem with strictly lower complexity than $\operatorname{SAT}\left(\left\{R_{1 / 3}^{\neq \neq \neq}\right\}\right)$. Let $R_{\mathrm{IN}_{2}}$ denote the weak base of $\mathrm{IN}_{2}$ in Table 3.1 from Chapter 3 and let $R_{2 / 4}=$ $\{(0,0,1,1),(0,1,0,1),(1,0,0,1),(1,1,0,0),(1,0,1,0),(0,1,1,0)\}$. It is not difficult to see that the relation obtained from $R_{2 / 4}$ by adding four complemented arguments, $R_{2 / 4}^{\neq \neq \neq \neq}$, is also a weak base of $I \mathbb{N}_{2}$, i.e., $\left\langle\left\{R_{2 / 4}^{\neq \neq \neq}\right\}\right\rangle_{\nexists}=$ $\left\langle\left\{R_{\mathrm{IN}_{2}}\right\}\right\rangle_{\nexists \ni}$. To see this, note that $R_{2 / 4}^{\neq \neq \neq \neq}$is simply $R_{\mathrm{IN}_{2}}$ with some of its arguments rearranged. We prefer to use the relation $R_{2 / 4}^{\neq \neq \neq}$instead of $R_{\mathrm{IN}_{2}}$ since $\operatorname{SAT}\left(\left\{R_{2 / 4}^{\neq \neq \neq \neq}\right\}\right)$is a slightly more natural satisfiability problem with a close relationship to $\operatorname{SAT}\left(\left\{R_{1 / 3}^{\neq \neq \neq}\right\}\right)$.

Hence, we now have two candidates for the easiest NP-complete SAT $(\cdot)$ problem: $\operatorname{SAT}\left(\left\{R_{1 / 3}^{\neq \neq \neq}\right\}\right)$and $\operatorname{SAT}\left(\left\{R_{2 / 4}^{\neq \neq \neq}\right\}\right)$. To prove that the former is not harder than the latter we have to give a CV-reduction from $\operatorname{SAT}\left(\left\{R_{1 / 3}^{\neq \neq \neq}\right\}\right)$ to $\operatorname{SAT}\left(\left\{R_{2 / 4}^{\neq \neq \neq}\right\}\right)$. By using clone theory it is not hard to prove that Theorem 7.3 is insufficient for this purpose since neither $R_{1 / 3}^{\neq \neq \neq} \in\left\langle\left\{R_{2 / 4}^{\neq \neq \neq}\right\}\right\rangle_{\nexists}$ nor $R_{2 / 4}^{\neq \neq \neq} \in\left\langle\left\{R_{1 / 3}^{\neq \neq \neq}\right\}\right\rangle_{\nexists \exists}$.

Theorem 7.7. $\left\langle\left\{R_{1 / 3}^{\neq \neq \neq}\right\}\right\rangle_{\nexists}$ and $\left\langle\left\{R_{2 / 4}^{\neq \neq \neq \neq}\right\}\right\rangle_{\nexists}$ are incomparable with respect to set inclusion.

Proof. We prove that neither of the weak partial co-clones can be a subset of the other.

For the first direction, assume towards contradiction that $\left\langle\left\{R_{1 / 3}^{\neq \neq \neq}\right\}\right\rangle_{\nexists} \subseteq$ $\left\langle\left\{R_{2 / 4}^{\neq \neq \neq \neq}\right\}\right\rangle_{\nexists \ni}$. Then $R_{1 / 3}^{\neq \neq \neq} \in\left\langle\left\{R_{2 / 4}^{\neq \neq \neq \neq}\right\}\right\rangle_{\nexists}$ which means that there exists a q.f.p.p. implementation of $R_{1 / 3}^{\neq \neq \neq}$in $\mathrm{IN}_{2}$. This is however impossible, since this implies $B R=I N_{2}$, violating the strict inclusion structure in Post's lattice.

For the other direction, assume towards contradiction that $\left\langle\left\{R_{2 / 4}^{\neq \neq \neq}\right\}\right\rangle_{\nexists} \subseteq$ $\left\langle\left\{R_{1 / 3}^{\neq \neq \neq}\right\}\right\rangle_{\nexists \text {. }}$. Then $\operatorname{pPol}\left(\left\{R_{1 / 3}^{\neq \neq \neq}\right\}\right) \subseteq \operatorname{pPol}\left(\left\{R_{2 / 4}^{\neq \neq \neq \neq}\right\}\right)$. We show that there exists a partial function $f$ with $f \in \operatorname{pPol}\left(\left\{R_{1 / 3}^{\neq \neq \neq}\right\}\right)$but $f \notin \operatorname{pPol}\left(\left\{R_{2 / 4}^{\neq \neq \neq \neq}\right\}\right)$, and hence that the initial premise was false. Let $f$ be the 4 -ary partial function defined only on tuples containing two 0 's and two 1's, and always returning 0 . This function does not preserve $R_{2 / 4}^{\neq \neq \neq}$as can be seen when applying it to the tuples $(0,1,0,1,1,0,1,0),(1,0,0,1,0,1,1,0),(0,1,1,0,1,0,0,1)$ and $(1,0,1,0,0,1,0,1)$ from $R_{2 / 4}^{\neq \neq \neq \neq}$. But we now show that $f$ preserves $R_{1 / 3}^{\neq \neq \neq}$, by showing that it is undefined on any sequence of four tuples from $R_{1 / 3}^{\neq \neq \neq}$. Indeed,

- taking three times the same tuple in such a sequence yields columns containing at least three 1 's or three 0 's,

- taking twice a tuple and twice another one always yields an all-0 and an all-1 column, and

- taking two different tuples, and twice the third one yields no balanced column except for one. 
Hence, $f$ preserves $R_{1 / 3}^{\neq \neq \neq}$since it is always undefined when applied to any sequence of tuples from $R_{1 / 3}^{\neq \neq \neq}$. Therefore $f$ is included in $\operatorname{pPol}\left(\left\{R_{1 / 3}^{\neq \neq \neq}\right\}\right)$but not in $\operatorname{pPol}\left(\left\{R_{2 / 4}^{\neq \neq \neq \neq}\right\}\right)$, as desired.

However, by introducing a constant number of fresh variables, it is possible to give a CV-reduction from $\operatorname{SAT}\left(\left\{R_{1 / 3}^{\neq \neq \neq}\right\}\right)$to $\operatorname{SAT}\left(\left\{R_{2 / 4}^{\neq \neq \neq \neq}\right\}\right)$.

Lemma 7.8. $\operatorname{SAT}\left(\left\{R_{1 / 3}^{\neq \neq \neq}\right\}\right)$is easier than $\operatorname{SAT}\left(\left\{R_{2 / 4}^{\neq \neq \neq \neq}\right\}\right)$.

Proof. We give a CV-reduction from $\operatorname{SAT}\left(\left\{R_{1 / 3}^{\neq \neq \neq}\right\}\right)$to $\operatorname{SAT}\left(\left\{R_{2 / 4}^{\neq \neq \neq \neq}\right\}\right)$. Let $\phi$ be an instance of $\operatorname{SAT}\left(\left\{R_{1 / 3}^{\neq \neq \neq}\right\}\right)$over a set of variables $V$ and let $C=$ $R_{1 / 3}^{\neq \neq \neq}\left(x_{1}, x_{2}, x_{3}, x_{4}, x_{5}, x_{6}\right)$ be an arbitrary constraint in $\phi$. Let $Y_{1}$ and $Y_{2}$ be two fresh variables not occurring in $V$. Then observe that the constraint $C^{\prime}=R_{2 / 4}^{\neq \neq \neq}\left(x_{1}, x_{2}, x_{3}, Y_{1} x_{4}, x_{5}, x_{6}, Y_{2}\right)$ is satisfiable if and only if $C$ is satisfiable, with $Y_{1}=1$ and $Y_{2}=0$. Let $\phi^{\prime}$ denote the instance of $\operatorname{SAT}\left(\left\{R_{2 / 4}^{\neq \neq \neq \neq}\right\}\right)$ resulting from repeating this translation for every constraint in $\phi$. We now claim that $\phi$ is satisfiable if and only if $\phi^{\prime}$ is satisfiable. If $\phi$ has a satisfying assignment $f$ then we obtain a satisfying assignment $f^{\prime}$ to $\phi^{\prime}$ by $f^{\prime}(x)=f(x)$ if $x \in V$ and $f\left(Y_{1}\right)=1$ and $f\left(Y_{0}\right)=0$. On the other hand, if $\phi^{\prime}$ has a satisfying assignment $f^{\prime}$ such that $f^{\prime}\left(Y_{0}\right)=1$ and $f^{\prime}\left(Y_{1}\right)=0$, then it is easy to see that the function $f(x)=1-f^{\prime}(x)$ for every $x \in V$ is a satisfying assignment to $\phi$, since $R_{2 / 4}^{\neq \neq \neq \neq}$is closed under complement. Hence, $\phi$ is satisfiable if and only if $\phi^{\prime}$ is satisfiable, and since the reduction only introduces two new variables, it follows that it is indeed a CV-reduction.

Since SAT $(S)$ is NP-complete if and only if $\langle S\rangle=\mathrm{BR}$ or $\langle S\rangle=\mathrm{IN}_{2}$, Lemma 7.6 together with Lemma 7.8 gives that $\operatorname{SAT}\left(\left\{R_{1 / 3}^{\neq \neq \neq}\right\}\right)$is the easiest NP-complete SAT $(S)$ problem.

Theorem 7.9. If $S \in \mathcal{H}$, then $\operatorname{SAT}\left(\left\{R_{1 / 3}^{\neq \neq \neq}\right\}\right)$is easier than $\operatorname{SAT}(S)$.

\subsection{Complexity Bounds for $\operatorname{SAT}\left(\left\{R_{1 / 3}^{\neq \neq \neq}\right\}\right)$and Related Problems}

As mentioned earlier, our notion of 'easiest problem' does not rule out the possibility that there exist languages which have the exact same time complexity as $\operatorname{SAT}\left(\left\{R_{1 / 3}^{\neq \neq \neq}\right\}\right)$. Proving that a problem $\operatorname{SAT}(S)$ is strictly easier than a problem $\operatorname{SAT}\left(S^{\prime}\right)$, i.e., that $\mathrm{T}\left(S^{\prime}\right)>\mathrm{T}(S)$, is of course in general much harder than giving a $C V$-reduction between the problems. In this section we relate the complexity between relations of the form $R^{\kappa \neq}$, where $R$ is $\kappa$-ary, and the language $\Gamma_{R}^{\text {ext }}$ obtained by expanding $R$ with all sign patterns.

For such relations we not only prove that $T\left(\left\{R^{\kappa \neq}\right\}\right)$ is strictly smaller than $\mathrm{T}\left(\Gamma_{R}^{\mathrm{ext}}\right)$, but that $\mathrm{T}\left(\Gamma_{R}^{\mathrm{ext}}\right)=2 \mathrm{~T}\left(\left\{R^{\kappa \neq}\right\}\right)$. Said otherwise, we prove that under the $\operatorname{ETH}, \operatorname{SAT}\left(\left\{R^{\kappa \neq}\right\}\right)$ is solvable in time $2^{(c+\varepsilon)|V|}$ for all $\varepsilon>0$, if and only if $\operatorname{SAT}\left(\left\{\Gamma_{R}^{\text {ext }}\right\}\right)$ is solvable in time $2^{(2 c+\varepsilon)|V|}$ for all $\varepsilon>0$. This gives tight bounds of the complexity of relations containing a sufficient number of complementary arguments and languages containing all sign patterns. 


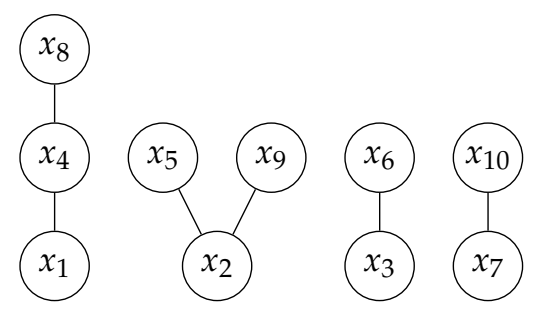

Figure 7.1: The complement graph $G(I)$ of the instance $I$ in Example 7.10.

We will in fact give a slightly more general proof. For this we need a few additional definitions. Let $R$ be a $\kappa$-ary Boolean relation, let $l \leq \kappa$ and let $R^{l \neq}\left(x_{1}, \ldots, x_{\kappa+l}\right)$ be a SAT $\left(\left\{R^{l \neq}\right\}\right)$ constraint. Say that $x_{i}$ and $x_{\kappa+i}, i \in$ $\{1, \ldots, l\}$, are complementary variables. Now given an instance $I=(V, C)$ of $\operatorname{SAT}\left(\left\{R^{l \neq}\right\}\right)$, we define the complement graph of $I$ to be the undirected graph $G(I)=(V, E)$, with $E=\left\{\{x, y\} \mid x, y\right.$ are complementary for some $\left.C_{i} \in C\right\}$. In other words, the vertices of $G(I)$ are the variables of $I$, and two variables have an edge between them if and only if they are complementary in some constraint in $C$.

Example 7.10. Let $I=(V, C)$ be an instance of $\operatorname{SAT}\left(\left\{R_{1 / 3}^{\neq \neq \neq}\right\}\right)$where $C=$ $\left\{R_{1 / 3}^{\neq \neq \neq}\left(x_{1}, x_{2}, x_{3}, x_{4}, x_{5}, x_{6}\right), R_{1 / 3}^{\neq \neq \neq}\left(x_{4}, x_{2}, x_{7}, x_{8}, x_{9}, x_{10}\right)\right)$. Then $G(I)=(V, E)$ where $E=\left\{\left\{x_{1}, x_{4}\right\},\left\{x_{4}, x_{8}\right\},\left\{x_{2}, x_{5}\right\},\left\{x_{3}, x_{6}\right\},\left\{x_{2}, x_{9}\right\},\left\{x_{7}, x_{10}\right\}\right\}$. This graph is visualised in Figure 7.5 Note that $G(I)$ has four connected components.

Lemma 7.11. Let $R^{l \neq}$ be a $(\kappa+l)$-ary Boolean relation, and let $\Gamma_{R}^{\mathrm{ext}}=\left\{R^{\left(s_{1}, \ldots, s_{\kappa}\right)} \mid\right.$ $\left.s_{1}, \ldots, s_{\kappa} \in\{-,+\}\right\}$. If there is a constant $c$ such that for all instances $I=(V, C)$ of $\operatorname{SAT}\left(\left\{R^{l \neq}\right\}\right.$, the number of connected components of $G(I)$ is at most $\frac{|V|}{c}$, then $\operatorname{SAT}\left(\left\{R^{l \neq}\right\}\right) L V$-reduces to $\operatorname{SAT}\left(\Gamma_{R}^{\text {ext }}\right)$ with parameter $\frac{c}{2}$.

Proof. Let $I=(V, C)$ be an instance of $\operatorname{SAT}\left(\left\{R^{l \neq}\right\}\right)$ and let $G(I)=(V, E)$ be the corresponding complement graph. We reduce $(V, C)$ to an equivalent instance $\left(V^{\prime}, C^{\prime}\right)$ of $\operatorname{SAT}\left(\left\{\Gamma_{R}^{\text {ext }}\right\}\right)$ with $\frac{|V|}{c}$ variables as follows. First, we choose one variable of $V$ per connected component in $G(I)$ and define $V^{\prime}$ to be the set of these. For each variable $x \in V$ we write $[x]$ for the representative of the connected component of $G(I)$ which contains $x$.

Observe that if there is an even-length (respectively odd-length) path between $x$ and $[x]$ in $G(I)$, then $x$ and $[x]$ must have the same value (respectively the opposite value) in all satisfying assignments of $I$. Moreover, if there is both an even-length and an odd-length path between $x$ and $[x]$, then $I$ must be unsatisfiable. Since this can be detected in time linear in the size of $I$, we henceforth assume that this is not the case.

Now let $R^{l \neq}\left(x_{i_{1}}, \ldots, x_{i_{\kappa}}, x_{i_{\kappa+1}}, \ldots, x_{i_{\kappa+l}}\right)$ be a constraint in $C$. We first replace each variable $x_{i_{j}}$ with $\left[x_{i_{j}}\right]$ (respectively $\overline{\left[x_{i_{j}}\right]}$ ) if there is an even-length (respectively odd-length) path between $x_{i_{j}}$ and $\left[x_{i_{j}}\right]$. We obtain an expression 
of the form $R^{l \neq}\left(\ell_{i_{1}}, \ldots \ell_{i_{\kappa}}, \ell_{i_{\kappa+1}}, \ldots, \ell_{i_{\kappa+l}}\right)$, and by construction, $\ell_{i_{j}}$ and $\ell_{i_{\kappa+j}}$ are opposite literals for all $j=1, \ldots, l$. Finally, from this expression we define the constraint $R^{s_{1}, \ldots, s_{\kappa}}\left(x_{i_{1}}^{\prime}, \ldots, x_{i_{\kappa}}^{\prime}\right)$, with for all $j, s_{j}=+$ and $x_{i_{j}}^{\prime}=x$ if $\ell_{i_{j}}$ is a positive literal $x$, and $s_{j}=-$ and $x_{i_{j}}^{\prime}=x$ if $\ell_{i_{j}}$ is a negative literal $\bar{x}$. We define $C^{\prime}$ to be the set of all such constraints.

Clearly, the resulting formula $\left(V^{\prime}, C^{\prime}\right)$ is an instance of $\operatorname{SAT}\left(\left\{\Gamma_{R}^{\text {ext }}\right\}\right)$, which contains at most $\frac{|V|}{c}$ variables by assumption, and which by construction is satisfiable if and only if $(V, C)$ is satisfiable. Hence, this construction is an $\operatorname{LV}$-reduction from $\operatorname{SAT}\left(\left\{R^{l \neq}\right\}\right)$ to $\operatorname{SAT}\left(\Gamma_{R}^{\text {ext }}\right)$ with parameter $\frac{1}{c}$.

For relations of the form $R^{\kappa \neq}$, where $\operatorname{ar}(R)=\kappa$, we get the following.

Lemma 7.12. Let $R$ be a $\kappa$-ary Boolean relation. Then for all instances $I=(V, C)$ of $\operatorname{SAT}\left(\left\{R^{\kappa \neq}\right\}\right.$, either I is trivially unsatisfiable, or the number of connected components of $G(I)$ is at most $\frac{|V|}{2}$.

Proof. Let $I=(V, C)$ be an instance of $\operatorname{SAT}\left(\left\{R^{\kappa \neq}\right\}\right)$. Since $R$ has arity $\kappa$, every $x \in V$ has at least one complementary variable $y$. If $x=y$ then $x$ is complementary to itself, which means that $I$ is trivially unsatisfiable. Otherwise we get that for each variable $x \in V$ there is at least one $y \in V, x \neq y$, such that $y$ occurs in the same connected component of $G(I)$. Hence, each connected component of $G(I)$ contains at least 2 variables, and the result follows.

We are now in position to give the main result of this section.

Theorem 7.13. Let $R^{\kappa \neq}$ be a $2 \kappa$-ary Boolean relation and let $\Gamma_{R}^{\mathrm{ext}}=\left\{R^{\left(s_{1}, \ldots, s_{\kappa}\right)} \mid\right.$ $\left.s_{1}, \ldots, s_{\mathcal{K}} \in\{-,+\}\right\}$. Then $\mathrm{T}\left(\Gamma_{R}^{\mathrm{ext}}\right)=2 \mathrm{~T}\left(\left\{R^{\kappa \neq}\right\}\right)$.

Proof. We prove that $\mathrm{T}\left(\left\{R^{\kappa \neq}\right\}\right) \leq \frac{\mathrm{T}\left(\Gamma_{R}^{\mathrm{ext}}\right)}{2}$ and that $\mathrm{T}\left(\Gamma_{R}^{\mathrm{ext}}\right) \leq 2 \mathrm{~T}\left(\left\{R^{\kappa \neq}\right\}\right.$. The former inclusion follows from Lemma 7.11 and 7.12. For the latter inclusion we give an $\operatorname{LV}$-reduction from $\operatorname{SAT}\left(\Gamma_{R}^{\text {ext }}\right)$ to $\operatorname{SAT}\left(\left\{R^{k \neq}\right\}\right)$ with parameter 2 .

Let $I=(V, C)$ be an instance of $\operatorname{SAT}\left(\Gamma_{R}^{\text {ext }}\right)$. For every $x_{i} \in V$ introduce a fresh variable $x_{i}^{\prime}$ and let $V^{\prime}$ be the resulting set of variables. Then, for every constraint $R^{\left(s_{1}, \ldots, s_{\kappa}\right)}\left(x_{i_{1}}, \ldots, x_{i_{\kappa}}\right)$ create a new constraint $R^{\kappa \neq}\left(y_{i_{1}}, \ldots, y_{i_{2 \kappa}}\right)$ where

- for $i_{j} \leq \kappa, y_{i_{j}}=x_{i_{j}}$ if $s_{j_{l}}=+$, and $y_{i_{j}}=x_{i_{j}}^{\prime}$ if $s_{j_{l}}=-$, and

- for $i_{j}>\kappa, y_{i_{j}}=x_{i_{j}-\kappa}$ if $s_{i_{j}-\kappa}=-$, and $x_{j_{l}}=x_{i_{j}-\kappa}^{\prime}$ if $s_{i_{j}-\kappa}=+$.

Let $C^{\prime}$ be the set of constraints resulting from this transformation. Then $\left(V^{\prime}, C^{\prime}\right)$ is satisfiable if and only if $(V, C)$ is satisfiable, and since $\left|V^{\prime}\right|=2|V|$, the reduction is an LV-reduction with parameter 2, which concludes the proof. 
Let $\Gamma_{R_{1 / 3}}^{\text {ext }}=\left\{R_{1 / 3}^{\left(s_{1}, s_{2}, s_{3}\right)} \mid s_{1}, s_{2}, s_{3} \in\{-,+\}\right\}$ be the language corresponding to 1-IN-3-SAT with all possible sign patterns. As is easily verified Lemmas 7.11 and 7.12 give an $\operatorname{LV}$-reduction from $\operatorname{SAT}\left(\left\{R_{1 / 3}^{\neq \neq \neq}\right\}\right)$to $\operatorname{SAT}\left(\Gamma_{R_{1 / 3}}^{\text {ext }}\right)$ with parameter $\frac{1}{2}$. This in turn implies not only that $\operatorname{SAT}\left(\left\{R_{1 / 3}^{\neq \neq \neq}\right\}\right)$is strictly easier than $\operatorname{SAT}\left(\Gamma_{R_{1 / 3}}^{\text {ext }}\right)$ (Lemma 6.2 but gives a precise bound on the difference in complexity between these two problems.

Corollary 7.14. $\mathrm{T}\left(\Gamma_{R_{1 / 3}}^{\mathrm{ext}}\right)=2 \mathrm{~T}\left(\left\{R_{1 / 3}^{\neq \neq \neq}\right\}\right)$.

We can give similar bounds for $\operatorname{SAT}\left(\left\{R_{2 / 4}^{\neq \neq \neq \neq}\right\}\right)$and $\operatorname{SAT}\left(\Gamma_{2 / 4}^{\text {ext }}\right)$, where $\Gamma_{2 / 4}^{\text {ext }}=\left\{R_{2 / 4}^{\left(s_{1}, s_{2}, s_{3}, s_{4}\right)} \mid s_{1}, s_{2}, s_{3}, s_{4} \in\{-,+\}\right\}$, i.e., $R_{2 / 4}^{\neq \neq \neq \neq}$expanded with all sign patterns.

Corollary 7.15. $\mathrm{T}\left(\Gamma_{2 / 4}^{\mathrm{ext}}\right)=2 \mathrm{~T}\left(\left\{R_{2 / 4}^{\neq \neq \neq \neq}\right\}\right)$.

Assuming the ETH is true it is proven in [IP01| that there exists an infinite increasing sequence of $\kappa, \kappa^{\prime}$ such that $3 \leq \kappa<\kappa^{\prime}$ and $\kappa$-SAT is strictly easier than $\kappa^{\prime}$-SAT. However, this result leaves quite a lot of gaps since it is difficult to estimate exactly how large these gaps in complexity are, and if this holds for any other languages besides $\kappa$-SAT. Our results are much more precise in the sense that we for every Boolean relation of the form $R^{\kappa \neq}$ can find a natural constraint language $S$ such that $\operatorname{SAT}\left(\left\{R^{\kappa \neq}\right\}\right)$ is strictly easier than $\operatorname{SAT}(S)$.

\subsection{Weak Partial Co-Clones Covering BR}

Since $\left\langle\left\{R_{1 / 3}^{\neq \neq \neq 01}\right\}\right\rangle_{\nexists}$ is the smallest element in the partial co-clone lattice covering BR it is natural to investigate other structural properties of this lattice. More formally, given a co-clone $\operatorname{Inv}(\mathrm{C})$, this question can be rephrased as determining the sublattice induced by the set of weak partial co-clones

$$
\operatorname{Int}_{\langle\cdot\rangle_{\nexists}}(\operatorname{Inv}(C))=\left\{\langle S\rangle_{\nexists \exists} \mid\langle S\rangle=\operatorname{Inv}(C)\right\} .
$$

Note that the set $\operatorname{Int}_{\langle\cdot\rangle_{\nexists}}(\operatorname{Inv}(C))$ is essentially just a relational reformulation of the set $\operatorname{Int}_{[\cdot]_{s}}(\mathrm{C})$ from Chapter 3 . Since $R_{1 / 3}^{\neq \neq \neq 01}$ is a weak base of BR it follows that $\left\langle\left\{R_{1 / 3}^{\neq \neq \neq 01}\right\}\right\rangle_{\nexists \exists}$ is the smallest element in the sublattice $\operatorname{Int}_{\langle\cdot\rangle_{\nexists}}(\mathrm{BR})$ while the largest element is simply the set of all Boolean relations, i.e. BR. Unfortunately, we cannot hope to fully describe this sublattice since it is of uncountably infinite cardinality [Sch15]. Hence, finite constraint languages are not expressive enough to fully characterise $B R$ or $I N_{2}$, as we remark in the following theorem.

Theorem 7.16. There is no finite $S$ such that $\langle S\rangle_{\nexists}=\mathrm{BR}$ or $\langle S\rangle_{\exists}=\mathrm{IN}_{2}$.

Proof. We only sketch the proof for the case of BR. Towards contradiction, assume that $S$ is finite and that $\langle S\rangle_{\nexists}=\mathrm{BR}$. Then $\operatorname{pPol}(S)=\operatorname{pPol}(\mathrm{BR})=$ 
$\{f \mid f$ is a subfunction of a projection function $\}=I_{2}$. In particular, we get that $\mathrm{pPol}(S)$ has finite order since it can be generated from any projection function, but this contradicts Theorem 4.30 in Chapter 4

Despite this we believe that even a partial classification of $\operatorname{Int}_{\langle\cdot\rangle_{\nexists}}(\mathrm{BR})$ could be of interest when comparing worst-case running times of NPcomplete SAT $(\cdot)$ problems. In the rest of this section we therefore provide such a partial classification, and in particular concentrate on languages corresponding to $\kappa$-SAT, MONOTONE 1 -IN- $\kappa$-SAT, and finite languages between MONOTONE 1-IN-3-SAT and $R_{1 / 3}^{\neq \neq \neq 01}$. To accomplish this we first introduce the constraint languages $\Gamma_{1 / k}=\left\{R_{1 / 1}, \ldots, R_{1 / k}\right\}$ and $\Gamma_{\mathrm{XSAT}}=\bigcup_{i=1}^{\infty} \Gamma_{1 / i}$.

The results are summarised in Figure 7.6 . That the inclusions are correct is proven in Section 7.6.1 and 7.6.2. We stress that this is indeed a partial classification. For example, for any relation $R$ such that $\left\langle\left\{R_{1 / 3}^{\neq \neq \neq 01}\right\}\right\rangle_{\nexists} \subset\langle\{R\}\rangle_{\nexists} \subset$ $\left\langle\Gamma_{1 / 3}\right\rangle_{\nexists}$, it holds that $\langle\{R\}\rangle_{\nexists} \subset\left\langle\left\{R, R_{1 / 2}\right\}\right\rangle_{\nexists} \subset\left\langle\Gamma_{1 / 3}\right\rangle_{\nexists}$ (since it is easy to prove that $R_{1 / 2} \notin\langle\{R\}\rangle_{\nexists}$ and $R_{1 / 1} \notin\left\langle\left\{R, R_{1 / 2}\right\}\right\rangle_{\nexists}$ ). It is also not difficult to find languages between $\left\langle\Gamma_{\mathrm{SAT}}^{\kappa}\right\rangle_{\nexists}$ and $\left\langle\Gamma_{\mathrm{SAT}}^{\kappa+1}\right\rangle_{\nexists}$ since for any $R \in \Gamma_{\mathrm{SAT}}^{\kappa+1}$ it holds that $\left\langle\Gamma_{\mathrm{SAT}}^{\kappa}\right\rangle_{\nexists} \subset\left\langle\Gamma_{\mathrm{SAT}}^{\kappa} \cup\{R\}\right\rangle_{\nexists} \subset\left\langle\Gamma_{\mathrm{SAT}}^{\kappa+1}\right\rangle_{\nexists}$. We discuss this in more detail in Section 7.9 .

The inclusions in Figure 7.6 are of particular importance when determining upper bounds on the complexities of $\operatorname{SAT}(\cdot)$ problems, since $\mathrm{T}(S) \leq$ $\mathrm{T}\left(S^{\prime}\right)$ if $\langle S\rangle_{\nexists} \subseteq\left\langle S^{\prime}\right\rangle_{\nexists \exists}$. With the help of the results from Section 7.5 we can in fact get tight bounds on the complexity for all languages below $\Gamma_{1 / 3}$.

Corollary 7.17. Let $S$ be a constraint language such that $\langle S\rangle=\mathrm{BR}$ and $\langle S\rangle_{\nexists} \subseteq$ $\left\langle\Gamma_{1 / 3}\right\rangle_{\nexists \text {. }}$. Then $\mathrm{T}\left(\left\{R_{1 / 3}^{\neq \neq \neq}\right\}\right) \leq \mathrm{T}(\mathrm{S}) \leq 2 \mathrm{~T}\left(\left\{\mathrm{R}_{1 / 3}^{\neq \neq \neq}\right\}\right)$.

Proof. The lower bound $\mathrm{T}\left(\left\{R_{1 / 3}^{\neq \neq \neq}\right\}\right) \leq \mathrm{T}(S)$ follows directly from Theorem 7.9 For the upper bound we note that for

$$
\Gamma_{R_{1 / 3}}^{\mathrm{ext}}=\left\{R_{1 / 3}^{\left(s_{1}, s_{2}, s_{3}\right)} \mid s_{1}, s_{2}, s_{3} \in\{-,+\}\right\},
$$

it holds that $\left\langle\Gamma_{R_{1 / 3}}^{\text {ext }}\right\rangle_{\nexists} \supseteq\left\langle\Gamma_{1 / 3}\right\rangle_{\nexists}$ and hence that $\langle S\rangle_{\nexists \exists} \subseteq\left\langle\Gamma_{R_{1 / 3}}^{\text {ext }}\right\rangle_{\nexists \exists}$. By applying Lemma 7.13 it then follows that $\mathrm{T}(S) \leq \mathrm{T}\left(\Gamma_{R_{1 / 3}}^{\mathrm{ext}}\right)=2 \mathrm{~T}\left(\left\{R_{1 / 3}^{\neq \neq \neq}\right\}\right)$.

Hence, even if we do not currently know whether the cardinality of the set $\left\{\langle S\rangle_{\nexists} \mid\langle S\rangle=\mathrm{BR},\langle S\rangle_{\nexists} \subseteq\left\langle\Gamma_{1 / 3}\right\rangle_{\nexists}\right\}$ is finite or infinite, we still obtain tight complexity bounds for all these languages with respect to $\mathrm{T}\left(\left\{R_{1 / 3}^{\neq \neq \neq}\right\}\right)$.

\subsubsection{Weak Partial Co-Clones below $\left\langle\Gamma_{1 / 3}\right\rangle_{\nexists}$}

We begin by explicating the structure of weak partial co-clones $\langle S\rangle_{\nexists\rceil}$ such that $\langle S\rangle_{\nexists} \subseteq\left\langle\Gamma_{1 / 3}\right\rangle_{\nexists}$. For this we introduce the relations $R_{1 / 3}^{\neq \neq 01}, R_{1 / 3}^{\neq 01}$ and $R_{1 / 3}^{01}$. Note that these relations are simply $R_{1 / 3}^{\neq \neq}, R_{1 / 3}^{\neq}$and $R_{1 / 3}$ with two additional constant columns adjoined.

Lemma 7.18. The following inclusions hold. 


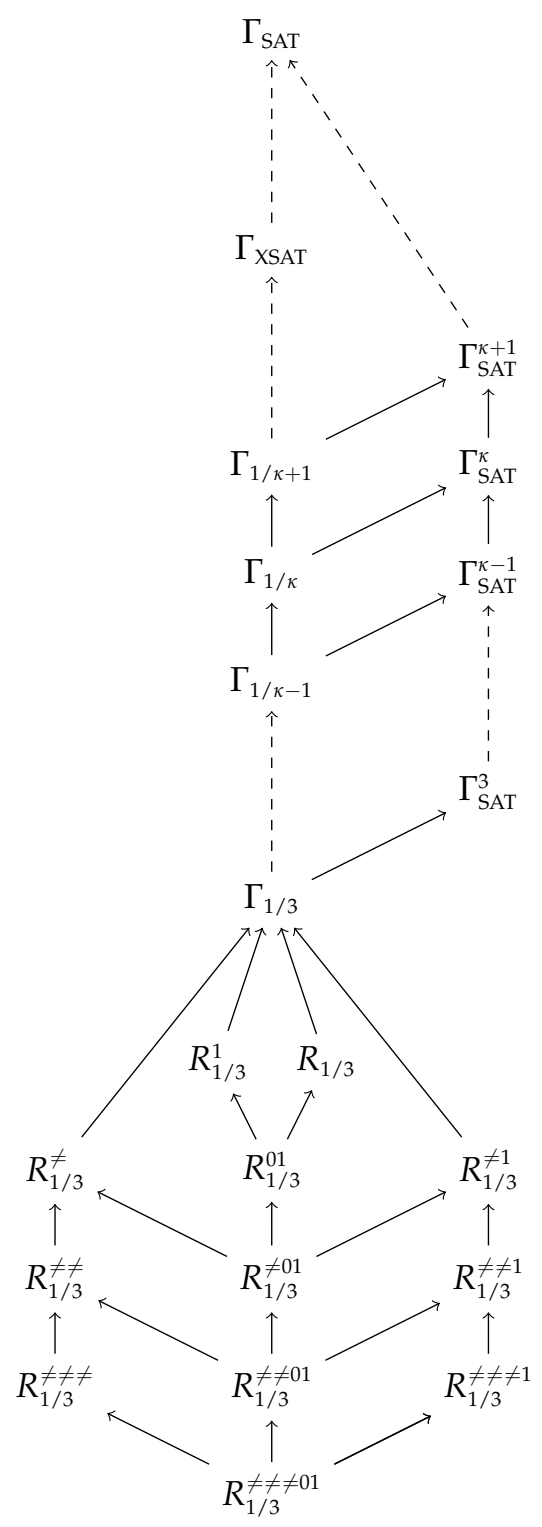

Figure 7.2: The structure of some weak partial co-clones in $\operatorname{Int}_{\langle\cdot\rangle_{\nexists}}(\mathrm{BR})$. A directed arrow from $S$ to $S^{\prime}$ means $\langle S\rangle_{\nexists} \subset\left\langle S^{\prime}\right\rangle_{\nexists}$ and hence also $\mathrm{T}(S) \leq \mathrm{T}\left(S^{\prime}\right)$. 

1. $\left\langle R_{1 / 3}^{\neq \neq \neq 01}\right\rangle_{\nexists} \subset\left\langle R_{1 / 3}^{\neq \neq 01}\right\rangle_{\nexists} \subset\left\langle R_{1 / 3}^{\neq 01}\right\rangle_{\nexists} \subset\left\langle R_{1 / 3}^{01}\right\rangle_{\nexists} \subset\left\langle R_{1 / 3}\right\rangle_{\nexists} \subset\left\langle\Gamma_{1 / 3}\right\rangle_{\nexists \prime}$
2. $\left\langle R_{1 / 3}^{01}\right\rangle_{\nexists} \subset\left\langle R_{1 / 3}^{1}\right\rangle_{\nexists} \subset\left\langle\Gamma_{1 / 3}\right\rangle_{\nexists}$,
3. $\left\langle R_{1 / 3}^{\neq \neq \neq}\right\rangle_{\nexists} \subset\left\langle R_{1 / 3}^{\neq \neq}\right\rangle_{\nexists} \subset\left\langle R_{1 / 3}^{\neq}\right\rangle_{\nexists} \subset\left\langle\Gamma_{1 / 3}\right\rangle_{\nexists}$,
4. $\left\langle R_{1 / 3}^{\neq \neq 01}\right\rangle_{\nexists} \subset\left\langle R_{1 / 3}^{\neq \neq \neq}\right\rangle_{\nexists},\left\langle R_{1 / 3}^{\neq \neq 01}\right\rangle_{\nexists} \subset\left\langle R_{1 / 3}^{\neq \neq}\right\rangle_{\nexists},\left\langle R_{1 / 3}^{\neq 01}\right\rangle_{\nexists} \subset\left\langle R_{1 / 3}^{\neq}\right\rangle_{\nexists}$,
5. $\left\langle R_{1 / 3}^{\neq \neq \neq 1}\right\rangle_{\nexists} \subset\left\langle R_{1 / 3}^{\neq \neq 1}\right\rangle_{\nexists} \subset\left\langle R_{1 / 3}^{\neq 1}\right\rangle_{\nexists} \subset\left\langle\Gamma_{1 / 3}\right\rangle_{\nexists \prime}$
6. $\left\langle R_{1 / 3}^{\neq \neq 01}\right\rangle_{\nexists} \subset\left\langle R_{1 / 3}^{\neq \neq \neq 1}\right\rangle_{\nexists},\left\langle R_{1 / 3}^{\neq \neq 01}\right\rangle_{\nexists} \subset\left\langle R_{1 / 3}^{\neq \neq 1}\right\rangle_{\nexists \prime}\left\langle R_{1 / 3}^{\neq 01}\right\rangle_{\nexists} \subset\left\langle R_{1 / 3}^{\neq 1}\right\rangle_{\nexists}$.

Proof. We only show that the inclusions hold for the languages in (1) since the cases (2), (3), (4), (5) and (6) follow a very similar structure.

For each inclusion $\left\langle\left\{R_{1}\right\}\right\rangle_{\nexists} \subseteq\left\langle\left\{R_{2}\right\}\right\rangle_{\nexists}$ we prove that $R_{1} \in\left\langle\left\{R_{2}\right\}\right\rangle_{\nexists \exists}$, and hence also that $\left\langle\left\{R_{1}\right\}\right\rangle_{\nexists} \subseteq\left\langle\left\{R_{2}\right\}\right\rangle_{\nexists}$, by giving a q.f.p.p. definition of $R_{1}$ in

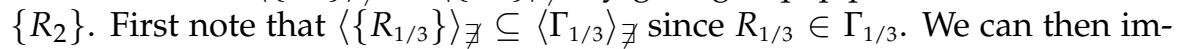
plement $R_{1 / 3}^{01}$ with $R_{1 / 3}$ by forcing $c_{0}$ and $c_{1}$ to be constant with an additional constraint, i.e.,

$$
R_{1 / 3}^{01}\left(x_{1}, x_{2}, x_{3}, c_{0}, c_{1}\right) \equiv R_{1 / 3}\left(x_{1}, x_{2}, x_{3}\right) \wedge R_{1 / 3}\left(c_{0}, c_{0}, c_{1}\right) .
$$

To implement $R_{1 / 3}^{\neq 01}$ with $R_{1 / 3}^{01}$ the procedure is similar but we also need to ensure that $x_{4}$ is assigned the opposite value of $x_{1}$, which can be done with the implementation

$$
R_{1 / 3}^{\neq 01}\left(x_{1}, x_{2}, x_{3}, x_{4}, c_{0}, c_{1}\right) \equiv R_{1 / 3}^{01}\left(x_{1}, x_{2}, x_{3}, c_{0}, c_{1}\right) \wedge R_{1 / 3}^{01}\left(x_{1}, x_{4}, c_{0}, c_{0}, c_{1}\right) .
$$

The proofs for $R_{1 / 3}^{\neq \neq 01}$ and $R_{1 / 3}^{\neq \neq 01}$ are entirely analogous.

To show a proper inclusion $\langle\{R\}\rangle_{\nexists} \subset\left\langle\left\{R^{\prime}\right\}\right\rangle_{\nexists}$ between every pair of relations $R$ and $R^{\prime}$ we provide a partial function $f$ which is a polymorphism of $R$ but not of $R^{\prime}$. Define the ternary minority function $f$ such that it maps any 3-tuple to the value that occurs least in it, or, in the case where all three arguments are equal, to the repeating value. For example $f(0,0,1)=1$ but $f(0,0,0)=0$. Observe that whenever $f$ is applied to three tuples with one or two repetitions it always yields one of these tuples. Hence, with the relations $R_{1 / 3}, R_{1 / 3}^{01}, R_{1 / 3}^{\neq 01}, R_{1 / 3}^{\neq \neq 01}, R_{1 / 3}^{\neq \neq 01}$, we only need to consider the case when $f$ is applied to the three distinct tuples in each relation. We now consider each of these pairs of relations in turn.

- $\left\langle\left\{R_{1 / 3}\right\}\right\rangle_{\nexists} \subset\left\langle\Gamma_{1 / 3}\right\rangle_{\nexists}$ : Let $f(1)=0$ and undefined otherwise. Then $f \notin \operatorname{pPol}\left(\Gamma_{1 / 3}\right)$ since $f$ does not preserve $R_{1 / 1}=\{(1)\}$. On the other hand $f$ does preserve $R_{1 / 3}$ since it will be undefined for any $t \in R_{1 / 3}$.

- $\left\langle\left\{R_{1 / 3}^{01}\right\}\right\rangle_{\nexists} \subset\left\langle\left\{R_{1 / 3}\right\}\right\rangle_{\nexists \exists}$ : Let $f_{1}$ be the partial ternary minority function which is undefined for the tuple $(0,0,0)$. Then $f_{1}$ does not preserve $R_{1 / 3}$ since the tuple $\left(f_{1}(0,0,1), f_{1}(0,1,0), f_{1}(1,0,0)\right)=(1,1,0) \notin R_{1 / 3}$. However, $f_{1}$ is a partial polymorphism of $R_{1 / 3}^{01}$ since in whatever order the three tuples $(0,0,1,0,1),(0,1,0,0,1)$ and $(1,0,0,0,1)$ from $R_{1 / 3}^{01}$ are taken $f_{1}$ will always be undefined for $(0,0,0)$. 
- $\left\langle\left\{R_{1 / 3}^{\neq 01}\right\}\right\rangle_{\nexists} \subset\left\langle\left\{R_{1 / 3}^{01}\right\}\right\rangle_{\nexists \exists}$ : The reasoning is similar as in the previous case except that we define a minority function $f_{2}$ which is undefined for the tuples $(1,1,0),(1,0,1)$ and $(0,1,1)$. As can be verified $f_{2}$ does not preserve $R_{1 / 3}^{01}$ since the tuple $\left(f_{1}(0,0,1), f_{1}(0,1,0), f_{1}(1,0,0), f_{1}(0,0,0)\right.$, $\left.f_{1}(1,1,1)\right)=(1,1,1,0,1) \notin R_{1 / 3}^{01}$. It is however a partial polymorphism of $R_{1 / 3}^{\neq 01}$ since, regardless of the order in which the tuples are taken in, it will be undefined for the fourth column which will always be one of $(1,1,0),(1,0,1)$ or $(0,1,1)$.

- $\left\langle\left\{R_{1 / 3}^{\neq \neq 01}\right\}\right\rangle_{\nexists \exists} \subset\left\langle\left\{R_{1 / 3}^{\neq 01}\right\}\right\rangle_{\nexists}$ : Define $f_{3}$ to be the ternary minority function except that it is undefined for $(1,0,1)$ and $(0,1,1)$. Then $f_{3}$ does not preserve $R_{1 / 3}^{\neq 01}$ since $\left(f_{3}(0,0,1), f_{3}(0,1,0), f_{3}(1,0,0), f_{3}(1,1,0), f_{3}(0,0,0)\right.$, $\left.f_{3}(1,1,1)\right)=(1,1,1,0,0,1) \notin R_{1 / 3}^{\neq 01}$. It will however always be undefined for the fourth or fifth column of $R_{1 / 3}^{\neq \neq 01}$ since one of these will be either $(1,0,1)$ or $(0,1,1)$.

- $\left\langle\left\{R_{1 / 3}^{\neq \neq \neq 01}\right\}\right\rangle_{\nexists} \subset\left\langle\left\{R_{1 / 3}^{\neq \neq 01}\right\}\right\rangle_{\nexists \exists}$ : The reasoning is the same as in the previous case but with a minority function $f_{4}$ undefined for $(0,1,1)$.

Observe that in the second part of the proof we exploit the Galois connection between strong partial clones and weak partial co-clones. This results in much more concise proofs since proving a strict inclusion $\left\langle\left\{R_{1}\right\}\right\rangle_{\exists} \subset$ $\left\langle\left\{R_{2}\right\}\right\rangle_{\exists}$ with relational tools is tantamount to the proof that it is impossible to find a q.f.p.p. definition of $R_{2}$ over $\left\{R_{1}\right\}$.

The inclusions in Lemma 7.18 do not rule out the possibility that some of the weak partial co-clones in Figure 7.6 collapse to a single weak partial co-clone. To rule out this we also need to prove that every pair of weak partial co-clones $\langle S\rangle_{\exists}$ and $\left\langle S^{\prime}\right\rangle_{\exists}$ which are not connected in Figure 7.6 are in fact incomparable with respect to set inclusion. Here we only sketch the proof that $\left\langle\left\{R_{1 / 3}^{\neq}\right\}\right\rangle_{\exists}$ and $\left\langle\left\{R_{1 / 3}\right\}\right\rangle_{\exists}$ are incomparable since the other cases are similar. First, define the ternary function $f$ such that $f(0,0,1)=$ $0, f(0,1,0)=1, f(1,0,0)=0, f(0,1,1)=0$, and let it be undefined otherwise. Then $f \in \operatorname{pPol}\left(\left\{R_{1 / 3}\right\}\right)$ (since if $t_{1}, t_{2}, t_{3} \in R_{1 / 3}$ then $f\left(t_{1}, t_{2}, t_{3}\right)$ is either undefined or yields a tuple in $\left.R_{1 / 3}\right)$ but $f \notin \operatorname{pPol}\left(\left\{R_{1 / 3}^{\neq}\right\}\right)$since $(f(1,0,0), f(0,1,0), f(0,0,1),(0,1,1))=(0,1,0,0) \notin R_{1 / 3}^{\neq}$. For the other direction define the ternary function $g$ such that $g(0,0,1)=g(0,1,0)=$ $g(1,0,0)=1$ but is undefined otherwise. A quick check shows that $g \in \operatorname{pPol}\left(\left\{R_{1 / 3}^{\neq}\right\}\right)$(since $g$ is always undefined for these tuples) but $g \notin$ $\operatorname{pPol}\left(\left\{R_{1 / 3}\right\}\right)$.

\subsubsection{Weak Partial Co-Clones above $\left\langle\Gamma_{1 / 3}\right\rangle_{\nexists}$}

In this section we focus on the constraint languages corresponding to MONOTONE $1-I N-\kappa-S A T$ and $\kappa$-SAT, and prove that both these languages form infinitely ascending chains of weak partial co-clones. We stress that these results hold independently of any complexity theoretical assumptions - the 
$\kappa$-SAT result was e.g. previously only known to hold if the ETH is true. At first, this result may be seen as further evidence that the ETH is plausible, but note that it does not contradict the possibility that the ETH is false, since the fact that $\left\langle\Gamma_{\mathrm{SAT}}^{\kappa}\right\rangle_{\nexists} \subset\left\langle\Gamma_{\mathrm{SAT}}^{\kappa+1}\right\rangle_{\nexists}$ does not necessarily imply that the running times of $\kappa$-SAT and $(\kappa+1)$-SAT differs.

Recall from Section 2.1 in Chapter 2 that we use $\overrightarrow{0}^{\kappa}$ to denote a $\kappa$-ary tuple of zeroes and $\overrightarrow{1}^{\kappa}$ to denote a $\kappa$-ary tuple of ones.

Theorem 7.19. $\left\langle\Gamma_{\mathrm{SAT}}^{\kappa}\right\rangle_{\nexists} \subset\left\langle\Gamma_{\mathrm{SAT}}^{\kappa+1}\right\rangle_{\nexists}$ for each $\kappa \geq 3$.

Proof. We begin by proving that $\left\langle\Gamma_{\text {SAT }}^{\kappa}\right\rangle_{\nexists} \subseteq\left\langle\Gamma_{\text {SAT }}^{\kappa+1}\right\rangle_{\nexists}$. By definition the language $\Gamma_{\text {SAT }}^{\kappa}$ contain all relations representable by the formulas $\left(x_{1} \vee \ldots \vee x_{\kappa}\right)$, $\left(\neg x_{1} \vee \ldots \vee x_{\kappa}\right), \ldots,\left(\neg x_{1} \vee \ldots \vee \neg x_{\kappa}\right)$, for all possible sign patterns. For simplicity we denote relations by their defining formulas and simply write $\left(\ell_{1} \vee \ldots \vee \ell_{\kappa}\right)$, where $\ell_{i}$ is a literal, for a relation in $\Gamma_{\mathrm{SAT}}^{\kappa}$. For example $\left(x_{1} \vee \ldots \vee x_{\kappa}\right)$ is the relation $\{0,1\}^{k} \backslash\left\{\overrightarrow{0}^{\kappa}\right\}$, and $\left(\neg x_{1} \vee \ldots \vee \neg x_{\kappa}\right)$ is the relation $\{0,1\}^{\kappa} \backslash\left\{\overrightarrow{1}^{\kappa}\right\}$.

It is then easy to prove that $\left\langle\Gamma_{\text {SAT }}^{\kappa}\right\rangle_{\nexists} \subseteq\left\langle\Gamma_{\text {SAT }}^{\kappa+1}\right\rangle_{\nexists}$ by giving explicit q.f.p.p. definitions of the relations in $\Gamma_{\mathrm{SAT}}^{\kappa}$ using $\Gamma_{\mathrm{SAT}}^{\kappa+1}$. For example, $\left(x_{1} \vee \ldots \vee x_{\kappa}\right) \equiv$ $\left(x_{1} \vee \ldots x_{\kappa-1} \vee x_{\kappa} \vee x_{\kappa}\right)$.

For the strict inclusion we give a function $f$ such that $f \in \operatorname{pPol}\left(\Gamma_{\mathrm{SAT}}^{\kappa}\right)$ but $f \notin \operatorname{pPol}\left(\Gamma_{\mathrm{SAT}}^{\kappa+1}\right)$, thus proving that $\mathrm{pPol}\left(\Gamma_{\mathrm{SAT}}^{\kappa+1}\right) \subset \operatorname{pPol}\left(\Gamma_{\mathrm{SAT}}^{\kappa}\right)$ and therefore that $\left\langle\Gamma_{\mathrm{SAT}}^{\kappa}\right\rangle_{\nexists} \subset\left\langle\Gamma_{\mathrm{SAT}}^{\kappa+1}\right\rangle_{\nexists \text { }}$. For each $\kappa \geq 3$ let the $\kappa$-ary function $f^{\kappa}$ be defined as $f^{\kappa}\left(x_{1}, \ldots, x_{\kappa}\right)=0$ if $\Sigma_{i=1}^{\kappa} x_{i}=1$, and undefined otherwise. We prove that for every $\kappa \geq 3$ it holds that $f^{\kappa+1} \in \operatorname{pPol}\left(\Gamma_{\text {SAT }}^{\kappa}\right)$ but $f^{\kappa+1} \notin \operatorname{pPol}\left(\Gamma_{\text {SAT }}^{\kappa+1}\right)$. Let $B\left(\Gamma_{\mathrm{SAT}}^{\kappa}\right)$ be the bounded base of $\Gamma_{\mathrm{SAT}}^{\kappa}$ from Theorem 5.23 in Chapter 5 . Recall that

$$
\mathcal{R}_{\kappa}\left(f^{\kappa+1}\right)=\left\{g \mid g \text { is a } \kappa^{\prime} \text {-restriction of } f^{\kappa+1} \text { for some } \kappa^{\prime} \leq \kappa\right\} .
$$

It is not difficult to see that every $g \in \mathcal{R}_{\kappa}\left(f^{\kappa+1}\right)$ is a partial projection function from which it follows that $\mathcal{R}_{\kappa}\left(f^{\kappa+1}\right) \subseteq B\left(\Gamma_{\mathrm{SAT}}^{\kappa}\right)$. We then apply Lemma 5.6 in Chapter 5 and conclude that $f^{\kappa+1} \in \operatorname{pPol}\left(\Gamma_{\mathrm{SAT}}^{\kappa}\right)$, since, trivially, $B\left(\Gamma_{\mathrm{SAT}}^{\kappa}\right) \subseteq$ $\operatorname{pPol}\left(\Gamma_{\mathrm{SAT}}^{\kappa}\right)$. The other direction, $f^{\kappa+1} \notin \mathrm{pPol}\left(\Gamma_{\mathrm{SAT}}^{\kappa+1}\right)$ follows immediately since $f^{\mathcal{K}+1}$ has arity $\kappa+1,\left|\operatorname{domain}\left(f^{\kappa+1}\right)\right|=\kappa+1$, and the fact that $f^{\mathcal{K}+1} \notin B\left(\Gamma_{\mathrm{SAT}}^{\kappa+1}\right)$ (since $f^{\kappa+1}$ by definition is not a partial projection function).

Next we prove that a similar separation result can be obtained for the sequence of $\Gamma_{1 / \kappa}$ languages.

Theorem 7.20. $\left\langle\Gamma_{1 / \kappa}\right\rangle_{\nexists} \subset\left\langle\Gamma_{1 /(\kappa+1)}\right\rangle_{\nexists}$ for each $\kappa \geq 1$.

Proof. Assume that $\kappa \geq 3$. Clearly, $\left\langle\Gamma_{1 / \kappa}\right\rangle_{\nexists} \subseteq\left\langle\Gamma_{1 /(\kappa+1)}\right\rangle_{\nexists}$ since $\Gamma_{1 / \kappa} \subseteq \Gamma_{1 /(\kappa+1)}$. For the proper inclusion let the $\kappa$-ary partial function $g^{\kappa}$ be defined as $g^{\kappa}\left(x_{1}, \ldots, x_{\kappa}\right)=0$ if $\Sigma_{i=1}^{\kappa} x_{i}=1$ and $g^{\kappa}\left(x_{1}, \ldots, x_{\kappa}\right)=1$ if $\left(x_{1}, \ldots, x_{\kappa}\right)=\overrightarrow{0}^{\kappa}$. The proof consists of two parts: first, we prove that $g^{\kappa+1} \in \operatorname{pPol}\left(\Gamma_{1 / \kappa}\right)$ for every $\kappa \geq 3$; second, we prove that $g^{\kappa} \notin \operatorname{pPol}\left(\Gamma_{1 / \kappa}\right)$ for every $\kappa \geq 3$.

Let $R_{1 / \kappa^{\prime}} \in \Gamma_{1 / \kappa}, \kappa^{\prime} \leq \kappa$. Note that $\left|R_{1 / \kappa^{\prime}}\right|=\kappa^{\prime}<\kappa+1$. Let $g \in \mathcal{R}_{\kappa^{\prime}}\left(g^{\kappa+1}\right)$. There are two cases to consider. Either $g$ is uncontractable to any arity $\leq \mathcal{K}^{\prime}$ 
in which case $g \in \mathcal{U}_{n}^{\kappa^{\prime \prime}}$ for every $\kappa^{\prime \prime} \leq \kappa^{\prime}$, and $g \in \operatorname{pPol}\left(\Gamma_{1 / \kappa}\right)$. Assume that $g$ is contractable to some arity $\kappa^{\prime \prime} \leq \kappa^{\prime}$. It is then not difficult to see that domain $(g)$ does not contain any exact covers of size $\left\{1, \ldots, \kappa^{\prime \prime}\right\}$, which according to Theorem 5.22 implies that $g \in \operatorname{pPol}\left(\left\{R_{1 / \kappa^{\prime}}\right\}\right)$. We then apply Lemma 5.6 and conclude that $g^{\kappa+1} \in \mathrm{pPol}\left(\Gamma_{1 / \kappa}\right)$. On the other hand, it is easy to see that $g^{\kappa} \notin \mathrm{pPol}\left(\Gamma_{1 / \kappa}\right)$ since the subfunction $g$ of $g^{\kappa}$ undefined on $\overrightarrow{0}^{\kappa}$ according to Theorem 5.22 is not included in the bounded base of $R_{1 / \kappa}$, since domain $(g)$ is an exact cover of $\{1, \ldots, \kappa\}$, but $g$ is not a partial projection.

Combining these two facts yields that $g^{\kappa+1} \in \operatorname{pPol}\left(\Gamma_{1 / \kappa}\right)$ but $g^{\kappa+1} \notin$ $\operatorname{pPol}\left(\Gamma_{1 /(\kappa+1)}\right)$. Hence, $\operatorname{pPol}\left(\Gamma_{1 / \kappa}\right) \supset \operatorname{pPol}\left(\Gamma_{1 /(\kappa+1)}\right)$ which implies that $\left\langle\Gamma_{1 / \kappa}\right\rangle_{\nexists} \subset\left\langle\Gamma_{1 /(\kappa+1)}\right\rangle_{\nexists}$.

It is easy to see that $\left\langle\Gamma_{1 / \kappa}\right\rangle_{\nexists} \subset\left\langle\Gamma_{\mathrm{SAT}}^{\kappa}\right\rangle_{\nexists \exists}$. To rule out the possibility that $\left\langle\Gamma_{1 /(\kappa+1)}\right\rangle_{\nexists} \subseteq\left\langle\Gamma_{\mathrm{SAT}}^{\kappa}\right\rangle_{\nexists}$ or $\left\langle\Gamma_{\mathrm{SAT}}^{\kappa}\right\rangle_{\nexists} \subseteq\left\langle\Gamma_{1 / \kappa}\right\rangle_{\nexists}$ we prove that these are in fact incomparable.

Theorem 7.21. $\left\langle\Gamma_{1 /(\kappa+1)}\right\rangle_{\nexists \exists}$ and $\left\langle\Gamma_{\mathrm{SAT}}^{\kappa}\right\rangle_{\nexists}$ are incomparable for each $\kappa \geq 3$.

Proof. We must prove that there exists partial functions $f, g$ such that $f \in$ $\operatorname{pPol}\left(\Gamma_{\mathrm{SAT}}^{\kappa}\right), f \notin \operatorname{pPol}\left(\Gamma_{1 /(\kappa+1)}\right)$ and $g \in \operatorname{pPol}\left(\Gamma_{1 /(\kappa+1)}\right)$ but $g \notin \operatorname{pPol}\left(\Gamma_{\mathrm{SAT}}^{\kappa}\right)$. For this let $f^{\kappa}$ and $g^{\kappa}$ be defined as in the proofs of Theorems 7.19 and 7.20 and recall that $f^{\kappa+1} \in \operatorname{pPol}\left(\Gamma_{\mathrm{SAT}}^{\kappa}\right)$ and $g^{\kappa+1} \in \operatorname{pPol}\left(\Gamma_{1 / \kappa}\right)$.

Let $\kappa \geq 3$. It is easy to see that $g^{\kappa+2} \notin \operatorname{pPol}\left(\Gamma_{\mathrm{SAT}}^{\kappa}\right)$ since $g^{\kappa+2}$ does not preserve the negative clause $\left(\neg x_{1} \vee \ldots \vee \neg x_{\kappa}\right)$ when applied to $\kappa+2$ tuples $t_{1}=\ldots=t_{\kappa+2}=\overrightarrow{0}^{\kappa}$. Similarly $f^{\kappa+1}$ does not preserve $R_{1 / \kappa+1} \in \Gamma_{1 /(\kappa+1)}$ since $f\left(t_{1}, \ldots, t_{\kappa+1}\right)=\overrightarrow{0}^{\kappa+1} \notin R_{1 / \kappa+1}$ when all $t_{1}, \ldots, t_{\kappa+1} \in R_{1 / \kappa+1}$ are distinct.

It is also not hard to prove that $\left\langle\Gamma_{\mathrm{XSAT}}\right\rangle_{\nexists}$ and $\left\langle\Gamma_{\mathrm{SAT}}^{\kappa}\right\rangle_{\nexists}$ are incomparable for each $\kappa \geq 3$. The direction $\left\langle\Gamma_{\mathrm{SAT}}^{\kappa}\right\rangle_{\nexists} \nsubseteq\left\langle\Gamma_{\mathrm{XSAT}}\right\rangle_{\nexists}$ follows from Theorem 7.21 . For the direction $\left\langle\Gamma_{\mathrm{XSAT}}\right\rangle_{\nexists} \not \subset\left\langle\Gamma_{\mathrm{SAT}}^{\kappa}\right\rangle_{\nexists}$ one can e.g. use the partial function $f(0)=1$, and undefined for 1 , since $f \in \operatorname{pPol}\left(\Gamma_{\mathrm{XSAT}}\right)$ but $f \notin \operatorname{pPol}\left(\Gamma_{\mathrm{SAT}}^{\kappa}\right)$.

The reader should note that in the proofs of Theorem 7.19 and Theorem 7.20 we used the results from Chapter 5 concerning bounded bases, which resulted in concise proofs. It would of course be possible to prove these theorems without this machinery, but the resulting proofs would be rather long-winded and mainly consist of exhaustive case analyses.

\subsection{LV-Reductions and Bounded-Degree Instances}

In this section we explicate a relationship between LV-reductions and degreebounded SAT(S)-DEG-B problems. In particular, as will be made clear in Section 7.8 when we relate $\operatorname{SAT}\left(\left\{R_{1 / 3}^{\neq \neq \neq}\right\}\right)$to the ETH, this relationship is very useful when proving that two problems are equally hard with respect to subexponential complexity. We also tackle the problem of determining the smallest $B$ such that SAT(S)-DEG- $B$ is NP-complete and provide these bounds for $R_{1 / 3}^{\neq \neq \neq}, R_{1 / 3}^{\neq \neq}, R_{1 / 3}^{\neq}$and $R_{1 / 3}$. 
For some of these proofs in this section it is convenient to consider p.p. definitions without equality constraints. More formally, we say that a relation $R$ has an equality-free primitive positive (e.f.p.p.) implementation over a constraint language $S$ if $R$ can be p.p. defined over $S$ without using any equality constraints. Note that if $\mathrm{Eq} \in S$ then e.f.p.p. and p.p. definitions over $S$ exactly coincide. These implementations will also prove to be useful in Section 7.8 where we relate the complexity of SAT $(\cdot)$ with the exponential-time hypothesis via sparsification.

Lemma 7.22. Let $S$ and $S^{\prime}$ be two finite constraint languages. If $S^{\prime} \subseteq\langle S\rangle$ and the equality relation $\mathrm{Eq}$ can be e.f.p.p. defined in $S$, then $\mathrm{SAT}\left(S^{\prime}\right)-D E G-B$ is $L V$ reducible to SAT $(S)-D E G-C$, where $C$ only depends on $S, S^{\prime}$, and $B$. The parameter of the $L V$-reduction depends only on $S, S^{\prime}$, and $B$.

Proof. Let $\phi$ be a SAT $\left(S^{\prime}\right)$-DEG- $B$-instance over a set of variables $V$. Since each variable can occur in at most $B$ constraints, there cannot be more than $|V| \cdot B$ constraints in total. Each such constraint is of the form $R\left(x_{1}, \ldots, x_{\kappa}\right)$ where $R \in S^{\prime}$. Since $S^{\prime} \subseteq\langle S\rangle$, the relation $R$ can then be expressed as a conjunction of constraints over $S \cup\{\mathrm{Eq}\}$ with a set of existentially quantified variables: $\exists y_{1}, \ldots, y_{l} . \bigwedge_{i=1}^{m} \psi_{i}\left(y_{i_{1}}, \ldots, y_{i_{\operatorname{ar}\left(\psi_{i}\right)}}\right)$, where each $\psi_{i} \in S \cup\{\mathrm{Eq}\}$ and $\left\{y_{i_{1}}, \ldots, y_{i_{\operatorname{ar}\left(\psi_{i}\right)}}\right\} \subseteq\left\{x_{1}, \ldots, x_{\kappa}\right\} \cup\left\{y_{1}, \ldots, y_{l}\right\}$. Furthermore, each Eqconstraint can be e.f.p.p. defined in $S$ so we may without loss of generality assume that Eq is not used in the definition.

Hence, the number of extra variables for each constraint depends only on the relations in $S^{\prime}$. Let $t$ denote the largest amount of variables that is required for implementing a constraint. In the worst case the total amount of new variables in the reduction is then $(|V| \cdot B) t$, which is linear with respect to $|V|$ since $B$ and $t$ are fixed values.

Since the reduction only increases the amount of variables with a constant factor it is indeed an LV-reduction, which concludes the lemma.

As we see in the following lemma, the condition that Eq is e.f.p.p. definable over $S$ is in fact always fulfilled when working with constraint languages in $\mathcal{H}$.

Lemma 7.23. If $S \in \mathcal{H}$, then $\mathrm{Eq}$ is e.f.p.p. implementable in $S$.

Proof. Let Neq denote the inequality relation $\{(0,1),(1,0)\}$. Since Neq is closed under complement, it follows that Neq can always be p.p. defined in $S$ (since $\langle S\rangle=\mathrm{BR}$ or $\left.\langle S\rangle=\mathrm{IN}_{2}\right)$. Let $N e q(x, y) \equiv \exists z_{1}, \ldots, z_{\kappa} \cdot \tau\left(x, y, z_{1}, \ldots, z_{\kappa}\right)$ denote the p.p. implementation with the least number of occurrences of Eqconstraints. Assume there exists an equality constraint $\operatorname{Eq}(v, w)$ in $\tau$. Clearly, this cannot be of the type $\operatorname{Eq}(x, y)$ since we are implementing $N e q(x, y)$. We can thus identify the variable $v$ with $w$ and still have a p.p. implementation of Neq - this violates the minimality condition. It follows that the implementation contains no Eq-constraint, i.e., it is an e.f.p.p. implementation. We 
can now e.f.p.p. implement Eq as follows since we are working over a twoelement domain:

$$
\operatorname{Eq}(x, y) \equiv \exists u . N e q(x, u) \wedge N e q(u, y)
$$

or equivalently the implementation

$$
\begin{array}{r}
\operatorname{Eq}(x, y) \equiv \exists u, z_{1}, \ldots, z_{\kappa}, w_{1}, \ldots, w_{\kappa} \cdot \tau\left(x, u, z_{1}, \ldots, z_{\kappa}\right) \wedge \\
\tau\left(u, y, w_{1}, \ldots, w_{\kappa}\right),
\end{array}
$$

which is clearly an e.f.p.p. implementation.

We get the following result by combining Lemmas 7.22 and 7.23 .

Corollary 7.24. Let $S, S^{\prime} \in \mathcal{H}$ such that $S^{\prime} \subseteq\langle S\rangle$ and let $B$ be an integer. Then, there exists an $L V$-reduction from $\operatorname{SAT}\left(S^{\prime}\right)-D E G-B$ to $\operatorname{SAT}(S)-D E G-C$ where $C$ only depends on $S, S^{\prime}$ and $B$.

In contrast to Lemma 7.29. Corollary 7.24 shows that there exists an $\mathrm{LV}$-reduction from SAT $(S)$-DEG- $B$ to $\operatorname{SAT}\left(\left\{R_{1 / 3}^{\neq \neq \neq}\right\}\right)$for every $S \in \mathcal{H}$ and arbitrary $B$. This apparently simple observation will be important in the next section where we study connections between $\operatorname{SAT}(\cdot)$ problems and the exponential-time hypothesis. Corollary 7.24 also suggests that for languages $S \in \mathcal{H}$ it is useful to know the largest $B$ such that $\mathrm{SAT}(S)$-DEG- $B$ is in P and the smallest $C$ such that $\operatorname{SAT}(S)$-DEG-C is NP-complete. For $\operatorname{SAT}\left(\left\{R_{1 / 3}\right\}\right)$ OCC $-B$ this value is already known since for $B=2$ it can be reduced to the problem of finding a perfect matching in a graph [Ist97], but for $B=3$ it is NP-complete even for planar instances [MR01]. Unsurprisingly the same also holds for $\operatorname{SAT}\left(\left\{R_{1 / 3}\right\}\right)$-DEG-2 as made clear in the following theorem.

Theorem 7.25. $\operatorname{SAT}\left(\left\{R_{1 / 3}\right\}\right)-D E G-2$ is solvable in polynomial time.

Proof. We show that $\operatorname{SAT}\left(\left\{R_{1 / 3}\right\}\right)$-DEG-2 is polynomial-time reducible to $\operatorname{SAT}\left(\left\{R_{1 / 3}\right\}\right)$-OCC-2. Let $I=(V, C)$ be an instance of $\operatorname{SAT}\left(\left\{R_{1 / 3}\right\}\right)$-DEG2 and let $|V|=n$. We transform $I$ into an instance $I^{\prime}$ of $\operatorname{SAT}\left(\left\{R_{1 / 3}\right\}\right)$-OCC-2 which is satisfiable if and only if $I$ is satisfiable.

For this we consider each variable which occurs at least twice in some constraint and at least one more time in another. Because $I$ is an instance of $\operatorname{SAT}\left(\left\{R_{1 / 3}\right\}\right)$-DEG-2, such a variable cannot occur in a third constraint; moreover, if a variable occurs only once in two different constraints, then it already has at most two occurrences. Observe that if a variable occurs three times in a constraint then the instance is trivially unsatisfiable, and since this can be detected in time linear in the size of $I$, we assume that this is not the case.

So let $C_{i} \in C$ be a constraint of the form $R_{1 / 3}\left(x_{i}, x_{i}, y_{i}\right)$, let $C_{j} \in C$ be a constraint where $x_{i}$ occurs in, and let $C_{k}$ be a constraint where $y_{i}$ occurs in. Since $R_{1 / 3}$ is a symmetric relation in the sense that $R_{1 / 3}(x, y, z) \equiv R_{1 / 3}(x, z, y) \equiv$ $R_{1 / 3}(y, x, z) \equiv R_{1 / 3}(y, z, x) \equiv R_{1 / 3}(z, x, y) \equiv R_{1 / 3}(z, y, x)$ we may without loss 
of generality assume that $x_{i}$ occurs in the first position of $C_{j}$ and that $y_{i}$ occurs in the first position of $C_{k}$. We perform a case study depending on $C_{j}$ and $C_{k}$ (if it exists).

First observe that for any assignment $f$ satisfying $I$ it holds that $f\left(x_{i}\right)=$ 0 and $f\left(y_{i}\right)=1$. Up to permutation of arguments there are a now a few different cases to consider:

1. $C_{j}=R_{1 / 3}\left(x_{i}, x_{j}, y_{i}\right)$ (and hence, $C_{k}$ does not exist),

2. $C_{j}=R_{1 / 3}\left(x_{i}, x_{i}, x_{j}\right), C_{k}=R_{1 / 3}\left(y_{i}, x_{k}, x_{k}\right)$,

3. $C_{j}=R_{1 / 3}\left(x_{i}, x_{i}, x_{j}\right), C_{k}=R_{1 / 3}\left(y_{i}, x_{k}, x_{k}^{\prime}\right)$,

4. $C_{j}=R_{1 / 3}\left(x_{i}, x_{j}, x_{j}^{\prime}\right), C_{k}=R_{1 / 3}\left(y_{i}, x_{j}, x_{j}\right)$,

5. $C_{j}=R_{1 / 3}\left(x_{i}, x_{j}, x_{j}^{\prime}\right), C_{k}=R_{1 / 3}\left(y_{i}, x_{j}, x_{k}\right)$,

6. $C_{j}=R_{1 / 3}\left(x_{i}, x_{j}, x_{j}^{\prime}\right), C_{k}=R_{1 / 3}\left(y_{i}, x_{k}, x_{k}\right)$,

7. $C_{j}=R_{1 / 3}\left(x_{i}, x_{j}, x_{j}^{\prime}\right), C_{k}=R_{1 / 3}\left(y_{i}, x_{k}, x_{k}^{\prime}\right)$,

where $x_{j}, x_{j}^{\prime}, x_{k}, x_{k}^{\prime}$ denote variables all distinct from each other and from $x_{i}$ and $y_{i}$. We have not included the cases that (1) trivially results in unsatisfiable instances or (2) is simply a permutation of case (1) - (7). For example $C_{j}=R_{1 / 3}\left(x_{i}, y_{i}, y_{i}\right)$ is unsatisfiable since $f\left(y_{i}\right)=1$ in any satisfying assignment $f$, and if $C_{j}=R_{1 / 3}\left(y_{i}, x_{i}, x_{i}\right)$ then this is simply a permutation of $R_{1 / 3}\left(x_{i}, x_{i}, y_{i}\right)$ and can safely be removed.

In each case it is easy to show how the constraints can be replaced by valid SAT $\left(\left\{R_{1 / 3}\right\}\right)$-OCC-2 constraints. In case (1) we introduce a fresh variable $x_{j}^{\prime}$ and replace $R_{1 / 3}\left(x_{i}, x_{j}, y_{i}\right)$ with $R_{1 / 3}\left(x_{j}^{\prime}, x_{j}, y_{i}\right)$. This ensures that $x_{i}$ and $y_{i}$ both occur only two times. In case (2) we introduce two fresh variables $x_{j}^{\prime}$ and $x_{k}^{\prime}$ and replace $C_{j}=R_{1 / 3}\left(x_{i}, x_{i}, x_{j}\right)$ and $C_{k}=R_{1 / 3}\left(y_{i}, x_{k}, x_{k}\right)$ with $R_{1 / 3}\left(x_{j}^{\prime}, x_{j}^{\prime}, x_{j}\right)$ and $R_{1 / 3}\left(y_{i}, x_{k}^{\prime}, x_{k}\right)$. Case (3) is very similar to case (2) and in case (4) we introduce a fresh variable $x_{j}^{\prime \prime}$ and replace $C_{k}=R_{1 / 3}\left(y_{i}, x_{j}, x_{j}\right)$ with $R_{1 / 3}\left(y_{i}, x_{j}^{\prime \prime}, x_{j}\right)$. For case (5) first note that $f\left(x_{i}\right)=f\left(x_{j}\right)=f\left(x_{k}\right)=0$ and that $f\left(y_{i}\right)=f\left(x_{j}^{\prime}\right)=1$ in any satisfying assignment $f$. To ensure that $x_{i}$ only occurs two times we introduce four fresh variables $z_{i}, z_{i}^{\prime}, z_{i}^{\prime \prime}, z_{i}^{\prime \prime \prime}$, and replace $C_{j}=R_{1 / 3}\left(x_{i}, x_{j}, x_{j}^{\prime}\right)$ and $C_{k}=R_{1 / 3}\left(y_{i}, x_{j}, x_{k}\right)$ with $R_{1 / 3}\left(z_{i}, z_{i}^{\prime}, z_{i}^{\prime}\right)$, $R_{1 / 3}\left(y_{i}, x_{j}, x_{k}\right), R_{1 / 3}\left(z_{i}, z_{i}^{\prime \prime}, z_{i}^{\prime \prime \prime}\right)$ and $R_{1 / 3}\left(z_{i}^{\prime \prime}, z_{i}^{\prime \prime \prime}, x_{j}^{\prime}\right)$. For any satisfying assignment $f$ it then holds that $f\left(x_{i}\right)=f\left(x_{j}\right)=f\left(x_{k}\right)=f\left(z_{i}^{\prime}\right)=f\left(z_{i}^{\prime \prime}\right)=f\left(z_{i}^{\prime \prime \prime}\right)=$ 0 , and $f\left(y_{i}\right)=f\left(x_{j}^{\prime}\right)=f\left(z_{i}\right)=1$.

In case (6) we replace $R_{1 / 3}\left(x_{i}, x_{j}, x_{j}^{\prime}\right)$ and $R_{1 / 3}\left(y_{i}, x_{k}, x_{k}^{\prime}\right)$ with the constraints $R_{1 / 3}\left(x_{i}^{\prime}, x_{j}, x_{j}^{\prime}\right), R_{1 / 3}\left(y_{i}, x_{i}^{\prime}, x_{i}^{\prime \prime}\right), R_{1 / 3}\left(y_{i}^{\prime}, x_{i}^{\prime \prime}, x_{i}^{\prime \prime \prime}\right), R_{1 / 3}\left(y_{i}^{\prime}, x_{k}, x_{k}^{\prime}\right)$, where $x_{i}^{\prime}, x_{i}^{\prime \prime}, x_{i}^{\prime \prime \prime}$ and $y_{i}^{\prime}$ are fresh variables. For any satisfying assignment $f$ it then holds that $f\left(x_{i}\right)=f\left(x_{i}^{\prime}\right)=f\left(x_{i}^{\prime \prime}\right)=f\left(x_{i}^{\prime \prime \prime}\right)=1-f\left(y_{i}\right)=1-f\left(y_{i}^{\prime}\right)$, and $f\left(x_{j}\right)=1-f\left(x_{j}^{\prime}\right)$. Last, the remaining case (7) is similar to case (6) with the exception that $x_{k}$ and $x_{k}^{\prime}$ only occurs one time in $C_{k}$. 
Repeating this procedure for every constraint then results in a satisfiability equivalent instance of $\operatorname{SAT}\left(\left\{R_{1 / 3}\right\}\right)$-OCC- 2 . Moreover, the reduction runs in polynomial time with respect to $n$ since the total number of constraints is bounded by $O\left(n^{3}\right)$, which concludes the proof.

It might be expected that the same holds for $\operatorname{SAT}\left(\left\{R_{1 / 3}^{\neq \neq \neq}\right\}\right), \operatorname{SAT}\left(\left\{R_{1 / 3}^{\neq \neq}\right\}\right)$ and $\operatorname{SAT}\left(\left\{R_{1 / 3}^{\neq}\right\}\right)$since they are all as easy as $\operatorname{SAT}\left(\left\{R_{1 / 3}\right\}\right)$. Contrary to intuition this is however not the case: $\operatorname{SAT}\left(\left\{R_{1 / 3}^{\neq \neq \neq}\right\}\right)$-DEG- $B$ is NP-complete even for $B=2$. This also holds for the relations $R_{1 / 3}^{\neq \neq}$and $R_{1 / 3}^{\neq}$. To prove this we first note that $R_{1 / 3}^{\neq \neq \neq}, R_{1 / 3}^{\neq \neq}$, and $R_{1 / 3}^{\neq}$are not $\Delta$-matroid relations.

Definition 7.26. ( $\Delta$-matroid relation) Let $R$ be a Boolean relation and $x, y, x^{\prime}$ be Boolean tuples of the same arity. Let $d(x, y)$ be the Hamming distance between $x$ and $y$. Then $x^{\prime}$ is a step from $x$ to $y$ if $d\left(x, x^{\prime}\right)=1$ and $d(x, y)=$ $d\left(x, x^{\prime}\right)+d\left(x^{\prime}, y\right) . R$ is a $\Delta$-matroid relation if it satisfies the following axiom: $\forall x, y \in R \forall x^{\prime}$. ( $x^{\prime}$ is a step from $x$ to $\left.y\right) \rightarrow\left(x^{\prime} \in R \vee \exists x^{\prime \prime} \in R\right.$ which is a step from $x^{\prime}$ to $\left.y\right)$.

Lemma 7.27. $R_{1 / 3}^{\neq}, R_{1 / 3}^{\neq \neq}$and $R_{1 / 3}^{\neq \neq \neq}$are not $\Delta$-matroid relations.

Proof. We begin with $R_{1 / 3}^{\neq \neq \neq}$. Let $x=(0,0,1,1,1,0)$ and $y=(0,1,0,1,0,1)$. These are both elements in $R_{1 / 3}^{\neq \neq \neq}$. Let $x^{\prime}=(0,0,0,1,1,0)$. Then $d\left(x, x^{\prime}\right)=1$ and $d(x, y)=d\left(x, x^{\prime}\right)+d\left(x^{\prime}, y\right)=1+3=4$, so $x^{\prime}$ is a step from $x$ to $y$. For $R_{1 / 3}^{\neq \neq \neq}$to be a $\Delta$-matroid relation we must have the following: either $x^{\prime} \in$ $R_{1 / 3}^{\neq \neq \neq}$, or there exists a $x^{\prime \prime}$ which is a step from $x^{\prime}$ to $y$. Since neither of the disjuncts are true, it follows that $R_{1 / 3}^{\neq \neq \neq}$is not a $\Delta$-matroid relation. For $R_{1 / 3}^{\neq \neq}$ and $R_{1 / 3}^{\neq}$the proofs are similar but using the tuples $(0,0,1,1,1),(0,1,0,1,0)$ and $(0,0,1,1),(1,0,0,0)$ as starting points instead.

The hardness results then follows from Theorem 3 in Dalmau and Ford [DF03], which states that SAT $(S)$-OCC-2 is NP-complete if $S$ contains a relation that is not a $\Delta$-matroid, and from the fact that SAT $(S)-O C C-2$ is a special case of SAT $(S)-D E G-2$.

Theorem 7.28. The following statements hold.

- $\operatorname{SAT}\left(\left\{R_{1 / 3}^{\neq}\right\}\right)-D E G-2$ is NP-complete.

- $\operatorname{SAT}\left(\left\{R_{1 / 3}^{\neq \neq}\right\}\right)-D E G-2$ is NP-complete.

- $\operatorname{SAT}\left(\left\{R_{1 / 3}^{\neq \neq \neq}\right\}\right)-D E G-2$ is NP-complete.

\subsection{The Exponential-Time Hypothesis}

Even though $\operatorname{SAT}\left(\left\{R_{1 / 3}^{\neq \neq \neq}\right\}\right)$is the easiest NP-complete $\operatorname{SAT}(\cdot)$ problem, we do not hope to prove or disprove that the problems $\operatorname{SAT}\left(\left\{R_{1 / 3}^{\neq \neq \neq}\right\}\right)$or $\operatorname{SAT}\left(\left\{R_{1 / 3}^{\neq \neq \neq}\right\}\right)$-DEG-2 are solvable in polynomial time, since this would settle the $\mathrm{P}=\mathrm{NP}$ question. A more assailable question is whether the problem can be solved in subexponential time. If yes, then we are none the wiser 
about $\mathrm{P} \stackrel{?}{=} \mathrm{NP}$; but if no, then $\mathrm{P} \neq \mathrm{NP}$. As a tool for studying subexponential problems, Impagliazzo et al. [IPZ01] proved a sparsification lemma for $\kappa-S A T$. Intuitively, the process of sparsification means that a SAT $(S)$ instance with a large number of constraints can be expressed as a disjunction of instances with a comparably small number of constraints. We prove that sparsification is possible not only for $\kappa$-SAT, but between all finite constraint languages $S$ and $S^{\prime}$ for which $\operatorname{SAT}(S)$ and $\operatorname{SAT}\left(S^{\prime}\right)$ are NP-complete, and use this to prove that $\operatorname{SAT}\left(\left\{R_{1 / 3}^{\neq \neq \neq}\right\}\right)$-DEG-2 is subexponential if and only if the exponential-time hypothesis is false. Due to sparsification we can also prove that all such SAT $(S)$ problems are subexponential if and only if one of them is subexponential (and that this holds also in the degree-bounded case), which is a significant refinement of Impagliazzo et al.'s result when restricted to finite Boolean constraint languages.

We first observe that LV-reductions are insufficient for these purposes since, under the assumption that coNP $\nsubseteq \mathrm{P} /$ poly, $\mathrm{SAT}\left(\Gamma_{\mathrm{SAT}}^{\kappa}\right)$ for $k \geq 4$ is not LV-reducible to $\operatorname{SAT}\left(\left\{R_{1 / 3}^{\neq \neq \neq}\right\}\right)$(recall that $\mathrm{P} /$ poly denotes the set of problems decidable by a Turing machine with a polynomially bounded advice function).

Lemma 7.29. There is no $L V$-reduction from $\operatorname{SAT}\left(\Gamma_{\mathrm{SAT}}^{\kappa}\right), k \geq 4$, to $\operatorname{SAT}\left(\left\{R_{1 / 3}^{\neq \neq \neq}\right\}\right)$ unless coNP $\subseteq P /$ poly (and the polynomial hierarchy collapses).

Proof. We assume that $k=4$ since the other cases are entirely similar. Assume (with the aim of reaching a contradiction) that there is an LV-reduction from $\operatorname{SAT}\left(\Gamma_{\mathrm{SAT}}^{4}\right)$ to $\operatorname{SAT}\left(\left\{R_{1 / 3}^{\neq \neq \neq}\right\}\right)$. We know that there is an LV-reduction (with parameter 1$)$ from $\operatorname{SAT}\left(\left\{R_{1 / 3}^{\neq \neq \neq}\right\}\right)$to $\operatorname{SAT}\left(\Gamma_{\mathrm{SAT}}^{3}\right)$ by Theorem 7.3 Taken together, this would imply the existence of an LV-reduction from SAT $\left(\Gamma_{\mathrm{SAT}}^{4}\right)$ to $\operatorname{SAT}\left(\Gamma_{\mathrm{SAT}}^{3}\right)$. Dell and van Melkebeek [DM10, Corollary 1] show that, under the hypothesis that coNP $\nsubseteq \mathrm{P}$ /poly, polynomial-time many-one reductions cannot reduce the density of $|V|$-variable $\mathrm{SAT}\left(\Gamma_{\mathrm{SAT}}^{d}\right)$ instances to $O\left(|V|^{c}\right)$ clauses for any constant $c<d$. Assume there is an LV-reduction (which is a restricted kind of polynomial-time many-one reduction) with parameter $C$ from $\operatorname{SAT}\left(\Gamma_{\mathrm{SAT}}^{4}\right)$ to $\operatorname{SAT}\left(\Gamma_{\mathrm{SAT}}^{3}\right)$. Let $\phi$ be an arbitrary instance (with $|V|$ variables) of $\operatorname{SAT}\left(\Gamma_{\mathrm{SAT}}^{4}\right)$ and $\phi^{\prime}$ the resulting $\operatorname{SAT}\left(\Gamma_{\mathrm{SAT}}^{3}\right)$ instance. The instance $\phi^{\prime}$ contains at most $C \cdot|V|+O(1)$ variables so it contains at most $O\left(8(C \cdot|V|)^{3}\right)=O\left(|V|^{3}\right)$ distinct constraints. This contradicts Dell \& van Melkebeek's result.

However, as we show in the forthcoming sections, this restriction can be overcome with the help of sparsification.

\subsubsection{Two Notions of Sparsification}

Sparsification in its original formulation by Impagliazzo et al. [IIZZ1] is only defined for $\kappa$-SAT formulas. We generalise the definition to arbitrary constraint languages, which leads to our first version of sparsification. 
Definition 7.30 (Sparsification $\left._{1}\right)$. Let $S$ and $S^{\prime}$ be two finite constraint languages. We say that $S$ is sparsifiable ${ }_{1}$ into $S^{\prime}$ if, for all $\varepsilon>0$ and for all SAT $(S)$ instances $\phi$ (with $|V|$ variables), there exists a set $\left\{\phi_{1}, \ldots, \phi_{t}\right\}$ of $\operatorname{SAT}\left(S^{\prime}\right)$ instances such that

1. $\phi$ is satisfiable if and only if at least one $\phi_{i}$ is satisfiable,

2. $\phi_{i}$ contains at most $C \cdot|V|$ constraints, where $C$ only depends on $\varepsilon, S$ and $S^{\prime}$,

3. $t \leq 2^{\varepsilon|V|}$, and

4. $\left\{\phi_{1}, \ldots, \phi_{t}\right\}$ can be computed in $O\left(\operatorname{poly}(|V|) \cdot 2^{\varepsilon|V|}\right)$ time.

Note that nothing in the definition says that $S$ and $S^{\prime}$ cannot be the same constraint language. If so, we simply say that $S$ is sparsifiable. Impagliazzo et al. |IPZ01| prove the following result for $\kappa$-SAT.

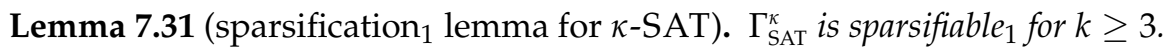

We will often use a slightly different formulation of sparsification which we denote sparsification 2 . In the second version, we focus on the degree of variables rather than the number of constraints.

Definition 7.32 (Sparsification 2 ). Let $S$ and $S^{\prime}$ be two finite constraint languages. We say that $S$ is sparsifiable 2 into $S^{\prime}$ if, for all $\varepsilon>0$ and for all $\operatorname{SAT}(S)$ instances $\phi$ (with $|V|$ variables), there exists a set $\left\{\phi_{1}, \ldots, \phi_{t}\right\}$ of $\operatorname{SAT}\left(S^{\prime}\right)$ instances such that

1. $\phi$ is satisfiable if and only if at least one $\phi_{i}$ is satisfiable,

2. $\phi_{i}$ is a $\operatorname{SAT}\left(S^{\prime}\right)$-DEG- $B$ instance, where $B$ only depends on $\varepsilon, S$ and $S^{\prime}$,

3. $\phi_{i}$ contains at most $D \cdot|V|$ variables, where $D$ only depends on $\varepsilon, S$ and $S^{\prime}$,

4. $t \leq 2^{\varepsilon|V|}$, and

5. $\left\{\phi_{1}, \ldots, \phi_{t}\right\}$ can be computed in $O\left(\operatorname{poly}(|V|) \cdot 2^{\varepsilon|V|}\right)$ time.

It should come as no surprise that sparsification $_{1}$ and sparsification $_{2}$ are tightly related concepts. It is easy to see that sparsification implies sparsification $_{1}$ : assume that every variable in $\phi_{i}$ has degree $\leq B$ and $\phi_{i}$ contains at most $D \cdot|V|$ variables. Then, a variable $x$ can appear in at most $B$ distinct constraints, and hence there can be at most $(B \cdot D) \cdot|V|$ distinct constraints in $\phi_{i}$.

It is also the case that sparsification implies sparsification $_{2}$ under a mild additional assumption. Recall from Section 7.7 that a relation $R$ is e.f.p.p. definable over a constraint language $S$ if $R$ can be p.p. defined over $S$ without using equality constraints. 
Lemma 7.33. Assume $S$ is sparsifiable ${ }_{1}$ into $S^{\prime}$. Then, $S$ is sparsifiable into $^{\prime} S^{\prime}$ if the equality relation is e.f.p.p. definable in $S^{\prime}$.

Proof. Assume $\operatorname{Eq}(x, y) \equiv \exists z_{1}, \ldots, z_{\kappa} \cdot \tau\left(x, y, z_{1}, \ldots, z_{\kappa}\right)$ is an e.f.p.p. implementation of Eq. Let $p$ denote the maximum degree of any variable in this implementation and let $q=\kappa+2$ denote the total number of variables. Let $\phi$ be a SAT $(S)$ instance and let $\left\{\phi_{1}, \ldots, \phi_{t}\right\}$ be a sparsification into $^{\prime}$ (for some $\varepsilon>0$ ). Let $\kappa^{\prime}$ be the maximum arity of the relations in $S^{\prime}$. Let us consider $\phi_{i}$ and assume $V$ is the set of variables occurring in $\phi_{i}$. Arbitrarily choose a variable $x \in V$ and assume that $x$ appears $m$ times in $\phi_{i}$. Introduce $m$ fresh variables $x_{1}, \ldots, x_{m}$ and the constraints

$$
\mathrm{Eq}\left(x_{1}, x_{2}\right), \mathrm{Eq}\left(x_{2}, x_{3}\right), \ldots, \mathrm{Eq}\left(x_{m-1}, x_{m}\right) .
$$

Finally, replace the $i$-th occurrence of $x$ in $\phi_{i}$ with the variable $x_{i}$. Do this for all variables $x \in V$ and let $\phi_{i}^{\prime}$ denote the resulting formula. Note the following:

1. The formula $\phi_{i}$ contains at most $C \cdot|V|$ constraints so it contains at most $C \cdot|V| \cdot \kappa^{\prime}$ variable occurrences. This implies that $\phi_{i}^{\prime}$ contains at most $\left(C \cdot \kappa^{\prime}\right) \cdot|V|+(C \cdot \kappa \cdot q) \cdot|V|$ variables where $(C \cdot \kappa \cdot q) \cdot|V|$ is an upper bound on the number of variables used for implementing the Eq constraints.

2. Each variable occurring in $\phi_{i}^{\prime}$ has degree at most $1+2 p$ : it appears in at most one position in the 'original constraints' and in $2 p$ positions in the constraints that e.f.p.p. implements Eq.

It follows that $\left\{\phi_{1}^{\prime}, \ldots, \phi_{t}^{\prime}\right\}$ is a sparsification 2 of $\phi$.

Whenever we study constraint languages in $\mathcal{H}$, we may actually view the two definitions as equivalent due to Lemma 7.23 Since $\Gamma_{\text {SAT }}^{\kappa} \in \mathcal{H}$ for $k \geq 3$ we immediately get the following.

Lemma 7.34. $\Gamma_{\mathrm{SAT}}^{\kappa}$ is sparsifiable 2 .

We also get the following simple connection between sparsification and subexponential complexity.

Lemma 7.35. Arbitrarily choose $S, S^{\prime} \in \mathcal{H}$ and assume that $S$ is sparsifiable 2 into $S^{\prime}$. If SAT $\left(S^{\prime}\right)-D E G-B$ is subexponential for all $B$, then $\operatorname{SAT}(S)$ is subexponential.

Proof. Assume that we want to solve SAT $(S)$ in time $O\left(\operatorname{poly}(|V|) \cdot 2^{|V| \varepsilon}\right)$ for some $\varepsilon>0$. For every $\alpha>0$ and $B$, there exists a time $O\left(\operatorname{poly}(|V|) \cdot 2^{|V| \alpha}\right)$ algorithm $A_{\alpha, B}$ for SAT $\left(S^{\prime}\right)$-DEG- $B$. Arbitrarily choose an instance $\phi$ of $\operatorname{SAT}(S)$ with $|V|$ variables. Sparsify $\phi$ with parameter $\varepsilon / 2$ into $\operatorname{SAT}\left(S^{\prime}\right)$ and let $\left\{\phi_{1}, \ldots, \phi_{t}\right\}$ be the resulting set of SAT $\left(S^{\prime}\right)$-DEG- $B$ instances (where $B$ is only dependent on $S, S^{\prime}$, and $\left.\varepsilon\right)$. This takes $O\left(\operatorname{poly}(|V|) \cdot 2^{(\varepsilon / 2)|V|}\right)$ time, 
$t \leq 2^{(\varepsilon / 2)|V|}$ holds, and each $\phi_{i}$ contains $C \cdot|V|$ variables for some constant $C$ that only depends on $\varepsilon$. Let $\alpha=\varepsilon / 2 C$ and apply $A_{\alpha, B}$ to the instances $\phi_{1}, \ldots, \phi_{t}$. If there is a satisfiable $\phi_{i}$, then $\phi$ is satisfiable, and otherwise it is not. The total time for this is $O\left(\operatorname{poly}(C \cdot|V|) \cdot t \cdot 2^{(\varepsilon / 2 C) \cdot C|V|)}\right)=$ $O\left(\operatorname{poly}(|V|) \cdot 2^{(\varepsilon / 2)|V|} \cdot 2^{(\varepsilon / 2)|V|}\right)=O\left(\operatorname{poly}(|V|) \cdot 2^{\varepsilon|V|}\right)$.

\subsubsection{Sparsification within $\mathcal{H}$}

In order to prove the general sparsification result (i.e., that all constraint languages $S, S^{\prime} \in \mathcal{H}$ can be sparsified ${ }_{2}$ into each other), we first prove that $\Gamma_{\mathrm{NAE}}^{\kappa}$ is sparsifiable ${ }_{2}$. In the following proofs we use $\gamma_{\mathrm{SAT}}^{\kappa}\left(\ell_{1}, \ldots \ell_{\kappa}\right), \ell_{i}=x_{i}$ or $\ell_{i}=\neg x_{i}$ (respectively $\left.\gamma_{\mathrm{NAE}}^{\kappa}\left(\ell_{1}, \ldots \ell_{\kappa}\right)\right)$ to denote constraints over $\Gamma_{\mathrm{SAT}}^{\kappa}($ respectively $\left.\Gamma_{\mathrm{NAE}}^{\kappa}\right)$.

Lemma 7.36 (sparsification 2 lemma for NAE-k-SAT). $\Gamma_{\mathrm{NAE}}^{\kappa}$ is sparsifiable 2.

Proof. Arbitrarily choose $\varepsilon>0$ and let $\phi$ be a SAT $\left(\Gamma_{\mathrm{NAE}}^{\kappa}\right)$ instance over the set of variables $V$. The proof proceeds in five steps.

1. $\phi$ is LV-reduced into a $\operatorname{SAT}\left(\Gamma_{\mathrm{SAT}}^{\kappa}\right)$ instance $\psi$,

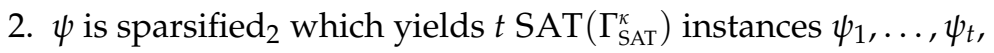

3. each $\psi_{i}$ is $\mathrm{LV}$-reduced into a $\mathrm{SAT}\left(\Gamma_{\mathrm{NAE}}^{\kappa+1}\right)$ instance $\psi_{i}^{\prime}$,

4. each $\psi_{i}^{\prime}$ is $\mathrm{LV}$-reduced into a $\operatorname{SAT}\left(\Gamma_{\mathrm{NAE}}^{\kappa+1}\right)$ instance $\psi_{i}^{\prime \prime}$ in which all variables have a bounded degree,

5. each $\psi_{i}^{\prime \prime}$ is $\mathrm{LV}$-reduced into a $\operatorname{SAT}\left(\Gamma_{\mathrm{NAE}}^{\kappa}\right)$ instance $\phi_{i}$.

Steps 1 and 2. Note that $\gamma_{\mathrm{NAE}}^{\kappa}\left(x_{1}, \ldots, x_{\kappa}\right)$ has the following q.f.p.p. implementation in terms of $\Gamma_{\mathrm{SAT}}^{\kappa}$ :

$$
\gamma_{\mathrm{NAE}}^{\kappa}\left(x_{1}, \ldots, x_{\kappa}\right) \equiv \gamma_{\mathrm{SAT}}^{\kappa}\left(x_{1}, \ldots, x_{\kappa}\right) \wedge \gamma_{\mathrm{SAT}}^{\kappa}\left(\neg x_{1}, \ldots, \neg x_{\kappa}\right) .
$$

In other words, if $\gamma_{\mathrm{NAE}}^{\kappa}\left(x_{1}, \ldots, x_{\kappa}\right)$ is a constraint from $\phi$, then it is satisfiable if and only if $\gamma_{\mathrm{SAT}}^{\kappa}\left(x_{1}, \ldots, x_{\kappa}\right) \wedge \gamma_{\mathrm{SAT}}^{\kappa}\left(\neg x_{1}, \ldots, \neg x_{\kappa}\right)$ is satisfiable. We can therefore form an equivalent $\operatorname{SAT}\left(\Gamma_{\mathrm{SAT}}^{\kappa}\right)$ instance $\psi$ by adding the complement of

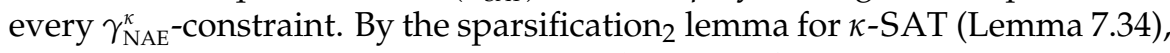
it follows that $\psi$ can be sparsified into $\left\{\psi_{1}, \ldots, \psi_{t}\right\}$.

Step 3. Arbitrarily choose $\psi_{i}, 1 \leq i \leq t$, and let $V_{i}$ denote the set of variables appearing in it. We begin by introducing one fresh variable $X$. For each constraint $\gamma_{\mathrm{SAT}}^{\kappa}\left(x_{1}, \ldots, x_{\kappa}\right) \in \psi_{i}$, we let $\gamma_{\mathrm{NAE}}^{\kappa+1}\left(x_{1}, \ldots, x_{\kappa}, X\right)$ be the corresponding $\gamma_{\mathrm{NAE}}^{\kappa+1}$-constraint, and we let $\psi_{i}^{\prime}$ be the resulting $\operatorname{SAT}\left(\Gamma_{\mathrm{NAE}}^{\kappa+1}\right)$ instance. Then, $\psi_{i}$ is satisfiable if and only if $\psi_{i}^{\prime}$ is satisfiable: if $\psi_{i}$ is satisfied by the assignment $f$, then $\psi_{i}^{\prime}$ is satisfied by the assignment $f^{\prime}$ defined such that $f^{\prime}(x)=f(x)$ if $x \in V_{i}$ and $f^{\prime}(X)=0$. For the other direction, assume $\psi_{i}^{\prime}$ is satisfied by the assignment $f^{\prime}$. If $f^{\prime}(X)=0$, then each constraint has at least one 
literal that is not assigned 0 by $f$, by which it follows that $\psi_{i}$ must be satisfiable. If $f^{\prime}(X)=1$, then we note that the complement $f^{\prime \prime}$ of $f^{\prime}$ defined as $f^{\prime \prime}(x)=1-f^{\prime}(x)$ is a satisfying assignment, too, and we can apply the same reasoning as above.

Since $\psi$ was sparsified ${ }_{2}$, the degree of the variables in $\psi_{i}$ is bounded by some constant $B$. It follows that the number of constraints in $\psi_{i}$ is at most $B \cdot\left|V_{i}\right|$ and, consequently, that the variable $X$ has at most degree $B \cdot\left|V_{i}\right|$ in $\psi_{i}^{\prime}$. Step 4 . Now, consider $\psi_{i}^{\prime}$ and let $W$ denote the degree of $X$. We continue by proving that the degree of $X$ can be reduced to a constant value. To do this, we e.f.p.p. implement the equality relation in $\Gamma_{\mathrm{NAE}}^{\kappa+1}$ :

$$
\mathrm{Eq}(x, y) \equiv \exists z \cdot \gamma_{\mathrm{NAE}}^{\kappa+1}(x, z, \ldots, z) \wedge \gamma_{\mathrm{NAE}}^{\kappa+1}(z, \ldots, z, y) .
$$

We see that the highest degree of any variable in this definition is $2 \kappa$.

To decrease the degree of $X$, we introduce $W$ fresh variables $X_{1}, \ldots, X_{W}$ and the following chain of equality constraints:

$$
\mathrm{Eq}\left(X_{1}, X_{2}\right) \wedge \mathrm{Eq}\left(X_{2}, X_{3}\right) \wedge \ldots \wedge \mathrm{Eq}\left(X_{W-1}, X_{W}\right) .
$$

Thereafter, we replace the occurrence of the variable $X$ in $\psi_{i}^{\prime}$ with the variable $X_{i}$. Let the resulting instance be $\psi_{i}^{\prime \prime}$ and note that $\psi_{i}^{\prime}$ is satisfiable if and only if $\psi_{i}^{\prime \prime}$ is satisfiable. Also note that the maximum degree $B^{\prime \prime}$ of any variable in $\psi_{i}^{\prime \prime}$ is bounded by $\max \{B, 2 \kappa, 3\}$ (where $B$ is the degree of variables in $V_{i}$, $2 \kappa$ is the degree of variables in the construction of $\mathrm{Eq}$, and 3 is the degree of the variables $\left.X_{1}, \ldots, X_{W}\right)$. Furthermore, we introduce no more than $2 W$ new variables-the variables $X_{1}, \ldots, X_{W}$ and one auxiliary variable per Eq constraint. Hence,

- $\psi_{i}^{\prime \prime}$ contains at most $\left|V_{i}\right|+2 W \leq\left|V_{i}\right|+2 \cdot B \cdot\left|V_{i}\right|=(2 B+1)\left|V_{i}\right|$ variables, and

- every variable in $\psi_{i}^{\prime \prime}$ has degree at most $B^{\prime \prime}$ for some $B^{\prime \prime}$ which depends only on $B$ and $\kappa$.

Step 5. We know that Eq is e.f.p.p. definable in $\Gamma_{\mathrm{NAE}}^{\kappa}$ (by the construction in Step 4) and we know that for each $\psi_{i}^{\prime \prime}$ no variable occurs more than $B^{\prime \prime}$ times. Consequently, we can apply Lemma 7.22 and LV-reduce $\psi_{i}^{\prime \prime}$ to an equivalent $\operatorname{SAT}\left(\Gamma_{\mathrm{NAE}}^{\kappa}\right)$-DEG-C instance $\phi_{i}$ for some $C$ that only depends on $B^{\prime \prime}$ and $k$. We now verify that the formula $\left\{\phi_{1}, \ldots, \phi_{t}\right\}$ is indeed a sparsification of $\phi$.

1. $\phi$ is satisfiable if and only if at least one $\phi_{i}$ is satisfiable. This is clear from the transformations above.

2. $\phi_{i}$ is a $\operatorname{SAT}\left(\Gamma_{\mathrm{NAE}}^{\kappa}\right)$-DEG-C instance, where $C$ only depends on $\varepsilon$ and $\kappa$. Tracing the proof backwards, we see that

a) $C$ depends only on $B^{\prime \prime}$ and $\kappa$,

b) $B^{\prime \prime}$ depends only on $B$ and $\kappa$, and 
c) $B$ depends only on $\varepsilon$ and $\kappa$.

3. $\phi_{i}$ contains at most $D^{\prime \prime} \cdot|V|$ variables where $D^{\prime \prime}$ only depends on $\varepsilon$ and $\kappa$. The instance $\psi_{i}^{\prime \prime}$ contains at most $(2 B+1) \cdot\left|V_{i}\right|$ variables where $\left|V_{i}\right| \leq D \cdot|V|$ for some $D$ that only depends on $\varepsilon$ and $\kappa$, and $B$ depends on $\varepsilon$ and $\kappa$ only. This implies that there exists a $D^{\prime}$ (that only depends on $\varepsilon$ and $\kappa$ ) such that the number of variables in $\phi_{i}$ is $\leq D^{\prime} \cdot D \cdot|V|$ by Lemma 7.22

4. $t \leq 2^{\varepsilon|V|}$. The transformation from $\phi$ to $\psi$ in step 1 does not introduce any new variables. Hence, the value $t$ is determined by the initial sparsification only and the bound follows from Lemma 7.34

5. $\left\{\phi_{1}, \ldots, \phi_{t}\right\}$ can be computed in $O\left(\operatorname{poly}(|V|) \cdot 2^{\varepsilon|V|}\right)$ time. We begin with a $\operatorname{SAT}\left(\Gamma_{\mathrm{NAE}}^{\kappa}\right)$ instance $\phi$ (with $|V|$ variables) and construct the $\operatorname{SAT}\left(\Gamma_{\mathrm{SAT}}^{\kappa}\right)$ instance $\psi$. This can clearly be done in linear time and the size of $\phi$ is linearly related to the size of $\psi$. In particular, $\phi$ contains as many variables as $\psi$. Thus, the sparsification 2 process takes $O\left(\operatorname{poly}(|V|) \cdot 2^{\varepsilon|V|}\right)$ time and each instance $\psi_{i}^{\prime}$ is only linearly larger than $\psi$. From this point, we only apply a series of LV-reductions to each $\psi_{i}^{\prime}$. Since they can be computed in time $O(\operatorname{poly}(|V|))$, the time bound follows.

By combining step 1 to step 5 we then get the required sparsification of $\Gamma_{\mathrm{NAE}}^{\kappa}$.

The following auxiliary lemma establishes that for any finite constraint language $S$ such that $S \subset$ BR (respectively $S \subset \mathrm{IN}_{2}$ ) it holds that $\operatorname{SAT}(S)$ is LV-reducible to $\kappa$-SAT (respectively NAE- $\kappa-S A T$ ) for some $\kappa$.

Lemma 7.37. Let $S$ be a finite constraint language such that $S \subset \mathrm{IN}_{2}$ (respectively $S \subset \mathrm{BR})$. Let $K$ denote the maximum arity of the relations in $S$. Then $\mathrm{SAT}(S)$ is $L V$-reducible (with parameter 1$)$ to $\operatorname{SAT}\left(\Gamma_{\mathrm{NAE}}^{K}\right)$ (respectively $\operatorname{SAT}\left(\Gamma_{\mathrm{SAT}}^{K}\right)$ ).

Proof. We give the proof for $S \subset \mathrm{IN}_{2}$; the case $S \subset \mathrm{BR}$ is analogous. Observe first that $\Gamma_{\mathrm{NAE}}^{\kappa} \subseteq\left\langle\Gamma_{\mathrm{NAE}}^{\kappa^{\prime}}\right\rangle_{\exists}$ for each $\kappa^{\prime} \geq \kappa$. This observation together with Theorem 4.2 from Section 4.2 in Chapter 4 implies that $S \subseteq\left\langle\Gamma_{\mathrm{NAE}}^{K}\right\rangle_{\exists}$. Since $S$ and $\left\langle\Gamma_{\mathrm{NAE}}^{K}\right\rangle_{\exists}$ are both finite we can then directly use Theorem 7.3 to deduce that $\operatorname{SAT}(S) \leq \mathrm{CV} \operatorname{SAT}\left(\Gamma_{\mathrm{NAE}}^{K}\right)$, and, hence, that $\operatorname{SAT}(S)$ is LV-reducible with parameter 1 to $\operatorname{SAT}\left(\Gamma_{\mathrm{NAE}}^{K}\right)$.

We can now prove that sparsification is possible between all $S, S^{\prime}$ in $\mathcal{H}$.

Theorem 7.38. (general sparsification) Arbitrarily choose $S, S^{\prime} \in \mathcal{H}$. Then, $S$ is sparsifiable $_{2}$ into $S^{\prime}$.

Proof. Throughout the proof, let $\kappa$ denote the highest arity of any relation in $S$. There are a few different cases depending on which co-clones are generated by $S$ and $S^{\prime}$ : 
1. $\langle S\rangle=\left\langle S^{\prime}\right\rangle=\mathrm{IN}_{2}$,

2. $\langle S\rangle=\left\langle S^{\prime}\right\rangle=\mathrm{BR}$,

3. $\langle S\rangle=\mathrm{IN}_{2},\left\langle S^{\prime}\right\rangle=\mathrm{BR}$

4. $\langle S\rangle=\mathrm{BR},\left\langle S^{\prime}\right\rangle=\mathrm{IN}_{2}$.

Case 1. Arbitrarily choose $\varepsilon>0$. Every $\operatorname{SAT}(S)$ instance $\phi$ with $|V|$ variables can be reduced to a $\operatorname{SAT}\left(\Gamma_{\mathrm{NAE}}^{\kappa}\right)$ instance $\phi^{\prime}$ with the same number of variables by Lemma7.37. We sparsify $y_{2}$ this instance according to Lemma7.36 and obtain the set $\left\{\phi_{1}^{\prime}, \ldots, \phi_{t}^{\prime}\right\}$. Each $\phi_{i}^{\prime}$ is an instance of SAT $\left(\Gamma_{\mathrm{NAE}}^{\kappa}\right)$-DEG-B for some $B$ that only depends on $S, \kappa$, and $\varepsilon$. By Lemma 7.23 the relation Eq is e.f.p.p. implementable in $\Gamma_{\mathrm{NAE}}^{\kappa}$. We can thus apply Lemma 7.22 (since $\left\langle S^{\prime}\right\rangle=\mathbb{I N}_{2}$ implies that $\Gamma_{\mathrm{NAE}}^{\kappa} \subseteq \mathbb{I N}_{2}=\left\langle S^{\prime}\right\rangle$ ) and LV-reduce this instance to an instance $\phi_{i}^{\prime \prime}$ of SAT $\left(S^{\prime}\right)$-DEG-C for some $C$ that only depends on $S, S^{\prime}$, and $B$. One can now easily verify that $\left\{\phi_{1}^{\prime \prime}, \ldots, \phi_{t}^{\prime \prime}\right\}$ is a sparsification of $\phi$.

Case 2. Analogous to Case 1 by using $\Gamma_{\mathrm{SAT}}^{\kappa}$ instead of $\Gamma_{\mathrm{NAE}}^{\kappa}$.

Case 3. By Lemma 7.37 it follows that SAT $(S)$ can be LV-reduced (with parameter 1) to $\operatorname{SAT}\left(\Gamma_{\mathrm{SAT}}^{\kappa}\right)$. Thereafter we can proceed as in Case 2.

Case 4. Arbitrarily choose $\varepsilon>0$. If $\phi$ is a SAT(S) instance with $|V|$ variables, it can be LV-reduced (with parameter 1) to a $\operatorname{SAT}\left(\Gamma_{\mathrm{SAT}}^{\kappa}\right)$ instance $\phi^{\prime}$ by Lemma 7.37. Sparsify 2 this instance (with parameter $\varepsilon$ ) into $\left\{\phi_{1}^{\prime}, \ldots, \phi_{t}^{\prime}\right\}$ according to Lemma 7.36. By recapitulating steps 3 and 4 from the proof of Lemma 7.36, we can then LV-reduce each $\phi_{i}^{\prime}$ to a SAT $\left(\Gamma_{\mathrm{NAE}}^{\alpha+1}\right)$-DEG- $B$ instance $\phi_{i}^{\prime \prime}$. We can now apply Lemma 7.22 (since $\left\langle S^{\prime}\right\rangle=\mathrm{IN}_{2}$ implies that $\left.\Gamma_{\mathrm{NAE}}^{\kappa+1} \subseteq \mathrm{IN}_{2}=\left\langle S^{\prime}\right\rangle\right)$ and LV-reduce this instance to an instance $\phi_{i}^{\prime \prime \prime}$ of SAT $\left(S^{\prime}\right)$ DEG-C for some $C$ that only depends on $S, S^{\prime}$, and $B$. It is now straightforward to verify that $\phi_{1}^{\prime \prime \prime}, \ldots, \phi_{t}^{\prime \prime \prime}$ is a sparsification ${ }_{2}$ of $\phi$.

Santhanam and Srinivasan [SS12] have shown that the unrestricted SAT problem (which corresponds to an infinite constraint language) does not admit sparsification to arbitrary finite constraint languages such that $\mathrm{SAT}(\cdot)$ is NP-complete. Consequently, it is a necessary condition that the constraint languages in Theorem 7.38 are indeed finite. Related questions are discussed by Dell and van Melkebeek [DM10].

\subsection{3 $\operatorname{SAT}(\cdot)$ and the Exponential-Time Hypothesis}

A related question to the exponential-time hypothesis is which problems can be proved to be subexponential if and only if $\kappa$-SAT is subexponential with the help of a size-preserving reduction. Impagliazzo et al. [IPZ01] prove that many NP-complete problems such as $\kappa$-colorability, clique and vertex cover are as hard as $\kappa$-SAT with respect to subexponential complexity. In this section, we prove that both $\operatorname{SAT}\left(\left\{R_{1 / 3}^{\neq \neq \neq}\right\}\right)$and $\operatorname{SAT}\left(\left\{R_{1 / 3}^{\neq \neq \neq}\right\}\right)$-DEG-2 are subexponential if and only if $\kappa$-SAT is subexponential, and, as a consequence, that this is true for all finite constraint languages $S$ for which $\operatorname{SAT}(S)$ is $\mathrm{NP}$-complete - even for degree-bounded instances. The following lemma 
together with the general sparsification result from the previous section are the crucial ingredients in the proof of the main result (Theorem 7.40).

Lemma 7.39. If $\operatorname{SAT}\left(\left\{R_{1 / 3}^{\neq \neq \neq}\right\}\right)-D E G-2$ is subexponential, then $\operatorname{SAT}\left(\left\{R_{1 / 3}^{\neq \neq \neq}\right\}\right)$is subexponential.

Proof. Recall that Neq denotes the inequality relation $\{(0,1),(1,0)\}$, and let $\mathrm{Eq}_{\kappa}$ denote the $\kappa$-ary equality relation $\{(0, \ldots, 0),(1, \ldots, 1)\}$.

Claim 1. $R_{1 / 3}^{\neq \neq \neq}$can e.f.p.p. implement $\operatorname{Neq}(x, y)$ such that every variable in the implementation has degree 1. Merely observe that $\operatorname{Neq}(x, y)$ has the e.f.p.p. implementation $\exists z, w, z^{\prime}, w^{\prime} \cdot R_{1 / 3}^{\neq \neq \neq}\left(x, z, w, y, z^{\prime}, w^{\prime}\right)$.

Claim 2. $R_{1 / 3}^{\neq \neq \neq}$can e.f.p.p. implement $\mathrm{Eq}_{3}\left(x_{1}, x_{2}, x_{3}\right)$ such that variables $x_{1}, \ldots, x_{3}$ have degree 1 and all other variables have degree at most 2 . Consider the following implementation:

$$
\begin{aligned}
\mathrm{Eq}_{3}\left(x_{1}, x_{2}, x_{3}\right) \equiv & \exists x_{1}^{\prime}, z, w, z^{\prime}, w^{\prime}, h_{1}, \ldots, h_{4}, h_{5} . \\
& \operatorname{Neq}\left(x_{1}, x_{1}^{\prime}\right) \wedge R_{1 / 3}^{\neq \neq \neq}\left(z, w, h_{1}, x_{1}^{\prime}, x_{2}, h_{2}\right) \wedge \operatorname{Neq}\left(z, z^{\prime}\right) \wedge \\
& \operatorname{Neq}\left(w, w^{\prime}\right) \wedge R_{1 / 3}^{\neq \neq \neq}\left(z^{\prime}, w^{\prime}, h_{3}, x_{3}, h_{4}, h_{5}\right) .
\end{aligned}
$$

It is straightforward to verify that it satisfies the degree bounds stated above. To see that it actually implements $\mathrm{Eq}_{3}$, first note that it is possible to assign the variables $z, w, z^{\prime}, w^{\prime}, h_{1}, \ldots, h_{5}$ values that satisfy the formula for every assignment $\sigma$ such that $\sigma\left(x_{1}\right)=\sigma\left(x_{2}\right)=\sigma\left(x_{3}\right)$. Next, we show that every satisfying assignment forces $x_{1}, x_{2}, x_{3}$ to take the same value. We first verify that an arbitrary satisfying assignment $\sigma$ satisfies $\sigma(z) \neq \sigma(w)$, i.e., $\sigma(z)=$ $1-\sigma(w)$. Assume $\sigma(z)=0$. Then, $\sigma\left(z^{\prime}\right)=1 \Rightarrow \sigma\left(w^{\prime}\right)=0 \Rightarrow \sigma(w)=1$. One can similarly show that if $\sigma(z)=1$, then $\sigma(w)=0$. Now let $\sigma$ be an arbitrary satisfying assignment. We see that

- $\sigma\left(x_{1}\right)=1-\sigma\left(x_{1}^{\prime}\right)=\sigma(z)=1-\sigma(w)=\sigma\left(x_{2}\right)$ and

- $\sigma\left(x_{3}\right)=1-\sigma\left(z^{\prime}\right)=\sigma(z)=1-\sigma\left(x_{1}^{\prime}\right)=\sigma\left(x_{1}\right)$.

This implies that $\sigma\left(x_{1}\right)=\sigma\left(x_{2}\right)=\sigma\left(x_{3}\right)$ and that we have an implementation of $\mathrm{Eq}_{3}$.

Claim 3. $R_{1 / 3}^{\neq \neq \neq}$can e.f.p.p. implement $\mathrm{Eq}_{\mathcal{\kappa}}\left(x_{1}, \ldots, x_{\kappa}\right)$ such that $x_{1}, \ldots, x_{\kappa}$ have degree 1 and all other variables have degree at most 2 . The case when $\kappa \leq 3$ follows from Claim 2. If we have an implementation of $\mathrm{Eq}_{\mathcal{\kappa}}$ satisfying the degree bounds, then

$$
\mathrm{Eq}_{\kappa+1}\left(x_{1}, \ldots, x_{\kappa+1}\right) \equiv \exists x^{\prime} \cdot \mathrm{Eq}_{\kappa}\left(x_{1}, \ldots, x_{\kappa-1}, x^{\prime}\right) \wedge \mathrm{Eq}_{3}\left(x^{\prime}, x_{\kappa}, x_{\kappa+1}\right)
$$

is a valid implementation that satisfies the degree bounds, too.

Arbitrarily choose $\varepsilon>0$ and let $\psi$ be an arbitrary instance of $\operatorname{SAT}\left(\left\{R_{1 / 3}^{\neq \neq \neq}\right\}\right)$with $|V|$ variables. Sparsify $2 \psi$ (with parameter $\varepsilon$ ) into $\left\{R_{1 / 3}^{\neq \neq \neq}\right\}$ according to Theorem 7.38 and let $\left\{\psi_{1}, \ldots, \psi_{t}\right\}$ be the result. Each $\psi_{i}$ is an instance of $\operatorname{SAT}\left(\left\{R_{1 / 3}^{\neq \neq}\right\}\right)$-DEG- $B$ where $B$ only depends on $\varepsilon$. Let $V_{i}$ be 
the set of variables in $\psi_{i}$. Assume that $x$ is a variable in $V_{i}$ that has degree $2<B^{\prime} \leq B$. Replace the $j$-th occurrence of $x$ with the fresh variable $x_{j}$. Finally, use the construction in Claim 3 and add the constraint $\mathrm{Eq}_{B^{\prime}}\left(x_{1}, \ldots, x_{B^{\prime}}\right)$. Do this for all variables occurring in $V_{i}$ and let $\psi_{i}^{\prime}$ denote the resulting instance. It is not hard to see that $\psi_{i}^{\prime}$ is indeed an instance of $\operatorname{SAT}\left(\left\{R_{1 / 3}^{\neq \neq \neq}\right\}\right)$-DEG-2. We need to calculate the number of variables in $\psi_{i}^{\prime}$. The variables in $\psi_{i}$ have at most degree $B$ so $\psi^{\prime}$ contains at most $B \cdot\left|V_{i}\right|$ variables if we do not count the variables introduced by the $\mathrm{Eq}_{\kappa}$ constraints. For simplicity, we assume that we have added $\left|V_{i}\right| \mathrm{Eq}_{B}$ constraints. Let $D$ be the number of variables in the implementation of $\mathrm{Eq}_{3}$ in Claim 2. $\mathrm{An}_{\mathrm{Eq}}$ constraint is constructed by 'chaining together' $B \mathrm{Eq}_{3}$ constraints-this is done by introducing $B-3$ new variables. Hence, one $\mathrm{Eq}_{B}$ constraint contains $B+(B-3)+B \cdot D$ variables. All in all, the instance $\psi_{i}$ contains at most $\left|V_{i}\right| \cdot(B+(B-3)+B \cdot D)+\left|V_{i}\right| \cdot B=\left|V_{i}\right| \cdot(B(D+3)-3)$ variables. We see that $B(D+3)-3$ depends only on $\varepsilon$ since $D$ is a fixed constant.

Assume now that we want to solve $\operatorname{SAT}\left(\left\{R_{1 / 3}^{\neq \neq \neq}\right\}\right)$in time $O($ poly $(|V|)$. $\left.2^{|V| \varepsilon}\right)$ for some $\varepsilon>0$. For every $\alpha>0$, there exists a time $O\left(\right.$ poly $\left.(|V|) \cdot 2^{|V| \alpha}\right)$ algorithm $A_{\alpha}$ for $\operatorname{SAT}\left(\left\{R_{1 / 3}^{\neq \neq \neq}\right\}\right)$-DEG-2. Arbitrarily choose an instance $\psi$ of $\operatorname{SAT}\left(\left\{R_{1 / 3}^{\neq \neq \neq}\right\}\right)$with $|V|$ variables. Apply the transformation described above with parameter $\varepsilon / 2$ and let $\left\{\psi_{1}, \ldots, \psi_{t}\right\}$ be the resulting set of $\operatorname{SAT}\left(\left\{R_{1 / 3}^{\neq \neq \neq}\right\}\right)$DEG-2 instances. This takes $O\left(\operatorname{poly}(|V|) \cdot 2^{(\varepsilon / 2)|V|}\right)$ time, $t \leq 2^{(\varepsilon / 2)|V|}$, and each $\psi_{i}$ contains $C \cdot|V|$ variables for some constant $C$ that only depends on $\varepsilon$. Let $\alpha=\varepsilon / 2 C$ and apply $A_{\alpha}$ to the instances $\psi_{1}, \ldots, \psi_{t}$. If there is a satisfiable $\psi_{i}$, then $\psi$ is satisfiable and, otherwise, not. This process takes time $O\left(\operatorname{poly}(C \cdot|V|) \cdot t \cdot 2^{(\varepsilon / 2 C) \cdot C|V|)}\right)=O\left(\operatorname{poly}(|V|) \cdot 2^{(\varepsilon / 2)|V|} \cdot 2^{(\varepsilon / 2)|V|}\right)=$ $O\left(\operatorname{poly}(|V|) \cdot 2^{\varepsilon|V|}\right)$.

This lemma can be viewed as yet another proof of NP-hardness for $\operatorname{SAT}\left(\left\{R_{1 / 3}^{\neq \neq \neq}\right\}\right)$-DEG-2. The transformation from $\psi_{i}$ to $\psi_{i}^{\prime}$ is a polynomial-time reduction from $\operatorname{SAT}\left(\left\{R_{1 / 3}^{\neq \neq \neq}\right\}\right)$-DEG- $B$ to $\operatorname{SAT}\left(\left\{R_{1 / 3}^{\neq \neq \neq}\right\}\right)$-DEG-2 (for any fixed $B)$, and $\operatorname{SAT}\left(\left\{R_{1 / 3}^{\neq \neq \neq}\right\}\right)$-DEG-B is NP-hard for some $B$ by Theorem 7.2

Theorem 7.40. The following statements are equivalent:

1. The exponential-time hypothesis is false.

2. $\operatorname{SAT}\left(\left\{R_{1 / 3}^{\neq}\right\}\right)-D E G-2 \in \mathrm{SE}$.

3. $\operatorname{SAT}\left(\left\{R_{1 / 3}^{\neq \neq}\right\}\right)-D E G-2 \in \mathrm{SE}$.

4. $\operatorname{SAT}\left(\left\{R_{1 / 3}^{\neq \neq \neq}\right\}\right)-D E G-2 \in \mathrm{SE}$.

5. $\operatorname{SAT}\left(\left\{R_{1 / 3}^{\neq \neq \neq}\right\}\right) \in \mathrm{SE}$.

6. For every $S \in \mathcal{H}, \operatorname{SAT}(S) \in \mathrm{SE}$.

7. For every $S \in \mathcal{H}$ and every $B, \operatorname{SAT}(S)-D E G-B \in \mathrm{SE}$.

8. There exists an $S \in \mathcal{H}$ such that $\operatorname{SAT}(S) \in \mathrm{SE}$. 
9. There exists an $S \in \mathcal{H}$ such that, for all $B, \mathrm{SAT}(S)-D E G-B \in \mathrm{SE}$.

Proof. 1 $\Longrightarrow 2$ If the exponential-time hypothesis is false then 3-SAT is subexponential. But then $\operatorname{SAT}\left(\left\{R_{1 / 3}^{\neq}\right\}\right)$must also be subexponential since it, according to the classification in Figure 7.6 is as easy as 3-SAT, which immediately implies that $\operatorname{SAT}\left(\left\{R_{1 / 3}^{\neq}\right\}\right)$-DEG-2 is subexponential.

$2 \Longrightarrow 3$. Assume that $\operatorname{SAT}\left(\left\{R_{1 / 3}^{\neq}\right\}\right)-$DEG-2 is subexponential. We give a size-preserving reduction from $\operatorname{SAT}\left(\left\{R_{1 / 3}^{\neq \neq}\right\}\right)$-DEG-2 to $\operatorname{SAT}\left(\left\{R_{1 / 3}^{\neq}\right\}\right)$DEG-2. Let $\phi$ be an instance of $\operatorname{SAT}\left(\left\{R_{1 / 3}^{\neq \neq}\right\}\right)$-DEG-2 with $|V|$ variables and $2|V|$ constraints. Then we replace every constraint $R_{1 / 3}^{\neq \neq}\left(x_{1}, x_{2}, x_{3}, x_{1}^{\prime}, x_{2}^{\prime}\right)$ with $R_{1 / 3}^{\neq}\left(x_{1}, x_{2}^{\prime \prime}, x_{3}, x_{1}^{\prime}\right) \wedge R_{1 / 3}^{\neq}\left(x_{2}, a, b, x_{2}^{\prime}\right) \wedge R_{1 / 3}^{\neq}\left(x_{2}^{\prime \prime}, a, b, x_{2}^{\prime \prime \prime}\right)$, where $x_{2}^{\prime}, x_{2}^{\prime \prime}, x_{2}^{\prime \prime \prime}, a$ and $b$ are fresh variables. As can be verified $\sigma\left(x_{2}^{\prime \prime}\right)=\sigma\left(x_{2}\right)=1-\sigma\left(x_{2}^{\prime}\right)$ for all models $\sigma$, and, furthermore, the degree of any newly introduced variables is at most two. If we repeat the procedure for every constraint in $\phi$ we get an equivalent instance $\phi^{\prime}$ of $\operatorname{SAT}\left(\left\{R_{1 / 3}^{\neq}\right\}\right)$-DEG-2 where the number of variables is bounded by $10|V|$.

$3 \Longrightarrow 4$ Same reduction as in the previous case.

$4 \Longrightarrow 5$ By Lemma 7.39

$5 \Longrightarrow 6$. Assume that $\operatorname{SAT}\left(\left\{R_{1 / 3}^{\neq \neq \neq}\right\}\right)$is subexponential. Clearly, $\operatorname{SAT}\left(\left\{R_{1 / 3}^{\neq \neq \neq}\right\}\right)$-DEG- $B$ is subexponential for all $B$, too. According to the general sparsification result (Theorem 7.38), $S$ can be sparsified into $\left\{R_{1 / 3}^{\neq \neq \neq}\right\}$. Hence, $\operatorname{SAT}(S)$ is subexponential by Lemma 7.35

$6 \Longrightarrow 7,6 \Longrightarrow 8,7 \Longrightarrow 9,8 \Longrightarrow 9$. Trivial.

$9 \Longrightarrow 1$ Assume that $\mathrm{SAT}(S)$-DEG- $B$ is subexponential for all $B$. By Theorem 7.38, $\operatorname{SAT}\left(\Gamma_{\mathrm{SAT}}^{3}\right)$ can be sparsified into $\operatorname{SAT}(S)$ so the implication follows from Lemma 7.35 .

When proving $5 \Longrightarrow 6$ we do not require that the instances have bounded degree. Hence, there may be a more direct way of proving the implication without using sparsification: one may for instance think of LVreductions from $\operatorname{SAT}(S)$ to $\operatorname{SAT}\left(\left\{R_{1 / 3}^{\neq \neq \neq}\right\}\right)$. We remind the reader that the existence of such reductions (for all $S \in \mathcal{H}$ ) are unlikely since they imply coNP $\subseteq \mathrm{P} /$ poly by Lemma 7.29

\subsection{Summary and Conclusions}

In this chapter we have shown that partial clone theory can be used as a framework to study the complexity of NP-complete problems, and applied this to the parameterized Boolean satisfiability problem. In particular this has allowed us to get a more nuanced view of the complexity of SAT $(\cdot)$ than possible with the algebraic approach in Jeavons [Jea98]. In other words studying the complexity of a problem with our approach provides much more information than merely stating that it is polynomial-time solvable or NP-complete. Throughout the chapter we have also seen that clone theoretical results in fact can yield highly practical applications. For example, the proof that $\operatorname{SAT}\left(\left\{R_{1 / 3}^{\neq \neq \neq}\right\}\right)$is the easiest NP-complete $\operatorname{SAT}(\cdot)$ problem is based 
on determining the largest element in an interval of strong partial clone a result that might otherwise be believed to be of purely theoretical interest. With this result we could also obtain a full understanding of the complexity of SAT $(S)$ with respect to subexponential complexity for all finite languages $S$. We therefore deem it likely that further investigations in partial clone theory, and in particular improving the classification in Section 7.6. can lead to a better understanding of the complexity differences between SAT $(\cdot)$ problems. 



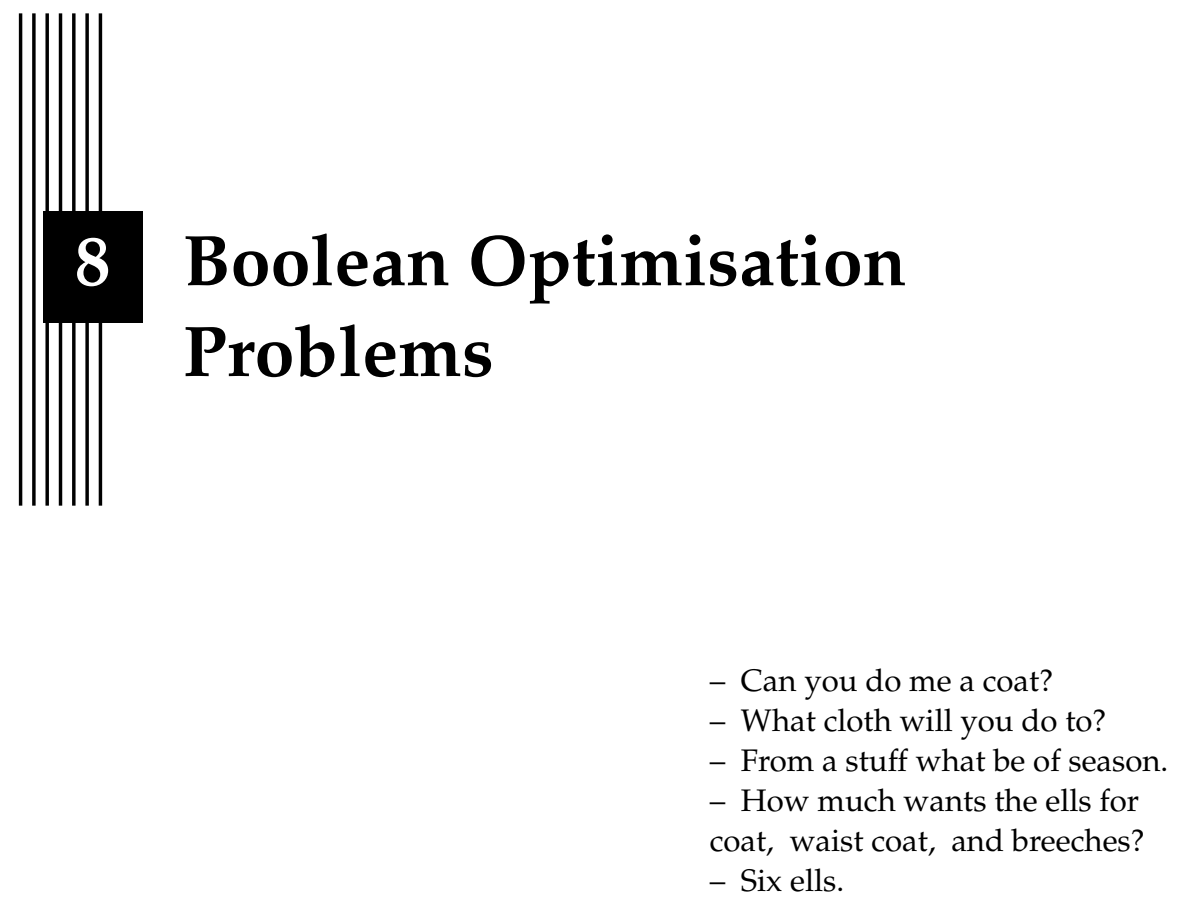

English as she is spoke

In this chapter we continue the work in Chapter7but in the context of the Boolean optimisation problems MAX-ONES $(\cdot)$ and $\operatorname{VCSP}(\cdot)$. Similar to the case of $\operatorname{SAT}(\cdot)$, we prove that we can obtain an 'easiest problem' for these two classes of problems, and use these results to give lower bounds with respect to the ETH.

\subsection{Introduction}

In the previous chapter we were able to isolate a relation $R_{1 / 3}^{\neq \neq \neq}$such that $\operatorname{SAT}\left(\left\{R_{1 / 3}^{\neq \neq \neq}\right\}\right)$resulted in the easiest NP-complete SAT $(\cdot)$ problem, and used this relation to give lower bounds of all NP-complete $\mathrm{SAT}(\cdot)$ problems in the context of the ETH. The proof strategy based on partial clone theory suggests a more general method to obtain an 'easiest problem' for any problem where the Galois connection in Theorem 2.21 is applicable. Assume that $X(\cdot)$ is a variable problem, as defined in Chapter 6 parameterized by a Boolean constraint language such that $X(S)$ is polynomial-time many-one reducible to 
$X\left(S^{\prime}\right)$ whenever $\operatorname{Pol}\left(S^{\prime}\right) \subseteq \operatorname{Pol}(S)$. Let $Y=\{\operatorname{Pol}(S) \mid X(S)$ is NP-complete $\}$. Then we have a method for obtaining the easiest $X(\cdot)$ problem. First, we compute the set $W=\left\{R_{\mathrm{IC}} \mid \mathrm{C} \in Y\right\}$, where each $R_{\mathrm{IC}}$ denotes the weak base of the co-clone IC from Table 3.1 in Chapter 3. Second, we try to prove that there exists $R_{\mathrm{IC}} \in W$ such that the problem $X\left(\left\{R_{\mathrm{IC}}\right\}\right)$ is CV-reducible to $X\left(\left\{R_{\mathrm{IC}}\right\}\right)$ for every other $R_{\mathrm{IC}} \in W$.

Hence, we have a general method to obtain the easiest problems, and in this chapter we apply this method and continue the hunt after the easiest $\mathrm{NP}$-complete problems in the context of MAX-Ones $(\cdot)$ and $\operatorname{VCSP}(\cdot)$. Recall from Chapter 6 that the MAX-ONES $(\cdot)$ problem [KSTW00] is a variant of $\operatorname{SAT}(\cdot)$ where the goal is to find a satisfying assignment which maximises the number of variables assigned the value 1 , and that the $\operatorname{VCSP}(\cdot)$ problem is a function minimisation problem that generalises the MAX-CSP and MIN-CSP problems [KSTW00]. We treat both the unweighted and weighted versions of these problems and use the prefix $U$ to denote the unweighted problem and $\mathrm{W}$ to denote the weighted version. These problems are well-studied with respect to separating tractable cases from NP-hard cases [KSTW00: TŽ13] but much less is known when considering the weaker schemes of LV-reductions and CV-reductions. We begin (in Section 8.3.1 by identifying the easiest language for W-MAX-ONES $(\cdot)$. This proof uses the strategy outlined in the preceding paragraph, but there are two complications. First, there exists an infinite number of Boolean co-clones resulting in NP-complete MAX-ONES $(\cdot)$ problems. We resolve this by utilising a certain inclusion structure of the weak bases computed in Chapter 3 and are able to remove all but seven relations. To show that exactly one of the resulting MAX-ONES $(\cdot)$ problems is CV-reducible to the other six we however require more powerful reduction techniques, based on weighted primitive positive implementations [JKT11. Tha10]. For $\operatorname{VCSP}(\cdot)$ the situation differs even more since the algebraic techniques developed for $\operatorname{CSP}(\cdot)$ and $\operatorname{SAT}(\cdot)$ are not applicable — instead we use multimorphisms [CCJK06] when considering the complexity of $\operatorname{VCSP}(\cdot)$. We prove (in Section 8.3.2 that the binary function $f_{\neq}$which returns 0 if its two arguments are different and 1 otherwise, results in the easiest NP-hard $\operatorname{VCSP}(\cdot)$ problem. This problem is very familiar since it is the MAX CUT problem slightly disguised. The complexity landscape surrounding these problems is outlined in Section 8.3.3.

With the aid of the languages identified in Section 8.3. we continue (in Section 8.4 by relating MAX-ONES and VCSP with LV-reductions and connect them with the ETH. Our results imply that (1) if the ETH is true then there cannot exist any NP-complete U-MAX-ONES(S), W-MAX-ONES $(S)$, or $\operatorname{VCSP}(\Delta)$ problems solvable in subexponential time and (2) that if the ETH is false then U-MAX-ONES $(S)$ and U-VCSP ${ }_{d}(\Delta)$ are solvable in subexponential time for every choice of $S$ and $\Delta$ and $d \geq 0$. Here $\mathrm{U}-\operatorname{VCSP}_{d}(\Delta)$ is the $\mathrm{U}-\operatorname{VCSP}(\Delta)$ problem restricted to instances where the sum to minimise contains at most $d n$ terms. Thus, to disprove the ETH, our result implies that it is sufficient to find a single language $S$ or a set of cost functions $\Delta$ such that 
U-MAX-Ones $(S)$, W-MAX-Ones $(S)$ or $\operatorname{VCSP}(\Delta)$ is NP-hard and solvable in subexponential time.

\subsection{Preliminaries}

We begin by formally defining the weighted and unweighted versions of MAX-ONES, and explain how partial clone theory can be used to study their worst-case time complexity.

W-MAX-ONES $(S)$

INSTANCE: A SAT $(S)$ formula $\phi$ over a set of variables $V$, a natural number $k \geq 0$, and $W=\left\{w_{1}, \ldots, w_{|V|}\right\}, w_{i} \in \mathbb{Q}_{\geq 0}$, a set of weights.

QUESTION: Does there exist a satisfying assignment $f$ to $\phi$ such that $\sum_{i=1}^{|V|} w_{i} f\left(x_{i}\right) \geq k ?$

The unweighted MAX-Ones $(S)$ problem (U-MAX-OnES $(S)$ ) is the W-MAX-Ones $(S)$ problem where all weights have the value 1 . For $\operatorname{MAX}-\operatorname{OnES}(S)$ we can employ a similar strategy as for SAT $(\cdot)$ in Chapter 7 since the complexity, up to polynomial-time reductions, is determined by $\operatorname{Pol}(S)$.

Theorem 8.1 (Khanna et al. [KSTW00]). Let $S$ and $S^{\prime}$ be finite Boolean constraint languages. If $\operatorname{Pol}\left(S^{\prime}\right) \subseteq \operatorname{Pol}(S)$, then (1) W-MAX-ONES $(S)$ is polynomialtime many-one reducible to $\mathrm{W}-\mathrm{MAX}-\mathrm{OnES}\left(S^{\prime}\right)$ and (2) $\mathrm{U}-\operatorname{MAX}-\mathrm{OnES}(S)$ is polynomial-time many-one reducible to $\mathrm{U}-\mathrm{MAX}-\mathrm{ONES}\left(S^{\prime}\right)$.

It is also not difficult to prove that $\mathrm{pPol}(S)$ determines the complexity of W-MAX-Ones $(S)$ up to $O\left(c^{|\operatorname{Var}(I)|}\right)$ time complexity. We omit the proof since it is entirely analogous to Theorem 7.3 in Chapter 7] The only complication is that, when removing equality constraints and identifying variables, one has to ensure that the resulting variable is given a weight which matches the sum of the weights of the variables which it has replaced.

Theorem 8.2. Let $S$ and $S^{\prime}$ be finite Boolean constraint languages. If $\mathrm{pPol}\left(S^{\prime}\right) \subseteq$ $\operatorname{pPol}(S)$, then $\mathrm{W}-\mathrm{MAX}-\mathrm{OnES}(S) \leq{ }^{\mathrm{CV}} \mathrm{W}-\mathrm{MAX}-\mathrm{OnES}\left(S^{\prime}\right)$.

This does not hold for the U-MAX-ONES $(\cdot)$ problem since one cannot safely remove equality constraints without using weights. However, recall that the weak bases in Chapter 3 are minimal in the sense that they can always be implemented without using equality constraints. We therefore obtain the following theorem.

Theorem 8.3. Let IC be a Boolean co-clone of finite order and let $R_{\mathrm{IC}}$ be the weak base of IC from Table 3.1 Then (1) U-MAX-ONES $\left(\left\{R_{\mathrm{IC}}\right\}\right) \leq \mathrm{CV}$ $\mathrm{U}-\mathrm{MAX}-\mathrm{ONES}(S)$ for any finite base $S$ of IC and (2) W-MAX-ONES $\left(\left\{R_{\mathrm{IC}}\right\}\right) \leq \mathrm{CV}$ W-MAX-ONES $(S)$ for any finite base $S$ of IC. 
We now turn our attention towards the valued constraint satisfaction problem over a set of finite-valued cost functions $\Delta(\operatorname{VCSP}(\Delta))$. Recall that an $n$-ary finite-valued cost function on $\mathbb{B}$ is a function from $\mathbb{B}^{n}$ to $\mathbb{Q}_{\geq 0}$.

\section{$\operatorname{VCSP}(\Delta)$}

INSTANCE: A set $V=\left\{x_{1}, \ldots, x_{n}\right\}$ of variables, a natural number $k \geq$ 0 , and the objective function $f_{I}\left(x_{1}, \ldots, x_{n}\right)=\sum_{i=1}^{q} w_{i} f_{i}\left(\mathbf{x}_{\mathbf{i}}\right)$ where, for every $1 \leq i \leq q, f_{i} \in \Delta, \mathbf{x}_{\mathbf{i}} \in V^{\operatorname{ar}\left(f_{i}\right)}$, and $w_{i} \in \mathbb{Q}_{\geq 0}$ is a weight.

QUESTION: Is there a function $h: V \rightarrow \mathbb{B}$ such that $f_{I}\left(h\left(x_{1}\right), \ldots, h\left(x_{n}\right)\right) \leq k ?$

We let U-VCSP be the VCSP problem without weights and U-VCSP $d$ (for $d \geq 0$ ) denote the U-VCSP problem restricted to instances containing at most $d|\operatorname{Var}(I)|$ constraints. Many optimisation problems can be viewed as $\operatorname{VCSP}(\Delta)$ problems for suitable $\Delta$ : well-known examples are the $\operatorname{MAX}-\mathrm{CSP}(S)$ and $\operatorname{MIN}-\mathrm{CSP}(S)$ problems where the number of satisfied constraints in a CSP instance are maximised or minimised (according to a set of weights). For each $S$, there obviously exists sets of cost functions $\Delta_{\min }, \Delta_{\max }$ such that $\operatorname{MIN}-\operatorname{CSP}(S)$ is polynomial-time equivalent to $\operatorname{VCSP}\left(\Delta_{\min }\right)$ and $\operatorname{MAx}-\operatorname{CSP}(S)$ is polynomial-time equivalent to $\operatorname{VCSP}\left(\Delta_{\max }\right)$. Observe that we have defined the problems U-VCSP, VCSP, U-MAX-ONES and W-MAX-ONES as decision problems rather than optimisation problems, and it is not difficult to see that they under this definition are NP-complete.

\subsection{The Easiest NP-complete MAX-ONES and VCSP Problems}

We will now study the complexity of W-MAX-ONES $(\cdot)$ and $\operatorname{VCSP}(\cdot)$ with respect to $\mathrm{CV}$-reductions. We prove that for both these problems there is a single language which is CV-reducible to every other NP-complete $\mathrm{W}-\mathrm{MAX}-\mathrm{ONES}(\cdot)$ or $\operatorname{VCSP}(\cdot)$ problem. Out of the infinite number of candidate languages generating different co-clones, we in Section 8.3.1 prove that the weak base $\left\{R_{\mathrm{II}_{2}}\right\}$ of BR defines the easiest W-MAX-ONES $(\cdot)$ problem. This might be contrary to intuition since one could be led to believe that the co-clones in the lower parts of the co-clone lattice, generated by very simple languages where the corresponding $\operatorname{SAT}(\cdot)$ problem is in $\mathrm{P}$, would result in even easier problems. For the $\operatorname{VCSP}(\cdot)$ problem we in Section 8.3.2 employ multimorphisms and prove that the function $f_{\neq}$defined as $f_{\neq}(0,0)=f_{\neq}(1,1)=1$ and $f_{\neq}(0,1)=f_{\neq}(1,0)=0$, results in the easiest NP-complete Boolean VCSP $(\cdot)$ problem. 


\subsubsection{The MAX-Ones Problem}

Here we use a slight reformulation of Khanna et al. 's [KSTW00] complexity classification of the MAX-ONES problem expressed in terms of polymorphisms. An operation $f$ is called arithmetical if $f(y, x, x)=f(y, x, y)=$ $f(x, x, y)=y$ for every $x, y \in \mathbb{B}$. The max function is defined as $\max (x, y)=$ 0 if $x=y=0$ and 1 otherwise.

Theorem 8.4 ([KSTW00]). Let $S$ be a finite Boolean constraint language. $\operatorname{MAx}-\operatorname{Ones}(S)$ is in $P$ if and only if $S$ is 1-closed, max-closed, or closed under an arithmetical operation.

The theorem holds for both the weighted and the unweighted version of the problem and showcases the strength of the algebraic method since it not only eliminates all constraint languages resulting in polynomialtime solvable problems, but also tells us exactly which cases remain, and which properties they satisfy. We will use Theorem 8.3 together with Theorem 8.4 in order to obtain an easiest NP-complete W-MAX-ONES $(\cdot)$ problem. That is, given a co-clone $\operatorname{Inv}(\mathrm{C})$ where $\mathrm{W}-\mathrm{MAX}-\mathrm{ONES}(\cdot)$ is NPcomplete, and a minimal weak base $R_{\mathrm{IC}}$ of $\operatorname{Inv}(\mathrm{C})$, we know that the problem W-MAX-ONES $\left(\left\{R_{\mathrm{IC}}\right\}\right)$ is the easiest W-MAX-ONES $(\cdot)$ problem for $\operatorname{Inv}(\mathrm{C})$ in the sense that it is CV-reducible to every W-MAX-ONES $(S)$ problem such that $\langle S\rangle=\operatorname{Inv}(\mathrm{C})$. Hence, to obtain an easiest $\mathrm{W}-$ MAX-OnES $(\cdot)$ problem, we only have to compare the complexity between W-MAX-ONES $\left(\left\{R_{I C}\right\}\right)$ and W-MAX-ONES $\left(\left\{R_{\mathrm{IC}}\right\}\right)$ for all weak bases $R_{\mathrm{IC}}$ and $R_{\mathrm{IC}}$ such that W-MAX-ONES $\left(\left\{R_{\mathrm{IC}}\right\}\right)$ and W-MAX-ONES $\left(\left\{R_{\mathrm{IC}}\right\}\right)$ are NP-complete.

Using Figure 2.1 from Chapter 2 in combination with Theorem 8.4 we however see that there is a slight complication with this strategy, since there exists an infinite number of weak bases to consider. In the following theorem we show how to circumvent this difficulty. We state this result for the U-MAX-ONES $(\cdot)$ problem since it is going to be used in Section 8.4 where we relate the complexity of U-MAX-ONES $(\cdot)$ to the ETH.

Theorem 8.5. U-MAX-ONES $(\{R\}) \leq \mathrm{CV}$ U-MAX-ONES $(S)$ for some $R \in\left\{R_{\mathrm{IS}_{1}^{2}}\right.$, $\left.R_{\mathrm{II}_{2}}, R_{\mathrm{IN}_{2}}, R_{\mathrm{IL}_{0}}, R_{\mathrm{IL}_{2}}, R_{\mathrm{IL}_{3}}, R_{\mathrm{ID}_{2}}\right\}$ whenever U-MAX-ONES(S) is NP-complete.

Proof. By Theorem 8.4 in combination with Table 2.1 and Figure 2.1 from Chapter 2 it follows that U-MAX-OnES $(S)$ is NP-complete if and only if $\langle S\rangle \supseteq \mathrm{IS}_{1}^{2}$ or if $\langle S\rangle \in\left\{\mathrm{IL}_{0}, \mathrm{IL}_{3}, \mathrm{IL}_{2}, \mathrm{IN}_{2}\right\}$. In principle we then for every coclone have to decide which language is $\mathrm{CV}$-reducible to every other base of the co-clone, but since a weak base always have this property, we can eliminate a lot of tedious work and directly consult the precomputed relations in Table 3.1 from Chapter 3 From this we first see that $\left\langle R_{\mathrm{IS}_{1}^{2}}\right\rangle_{\nexists} \subset\left\langle R_{\mathrm{IS}}^{\kappa}\right\rangle_{\nexists}$, $\left\langle R_{\mathrm{IS}_{12}^{2}}\right\rangle_{\exists} \subset\left\langle R_{\mathrm{IS}_{12}^{\kappa}}\right\rangle_{\nexists \prime}\left\langle R_{\mathrm{IS}_{11}^{2}}\right\rangle_{\nexists} \subset\left\langle R_{\mathrm{IS}_{11}^{\kappa}}\right\rangle_{\exists}$ and $\left\langle R_{\mathrm{IS}_{10}^{2}}\right\rangle_{\exists} \subset\left\langle R_{\mathrm{IS}}^{\kappa}\right\rangle_{10}$ for every $\kappa \geq 3$. Hence, in the four infinite chains $I S_{1}^{\mathcal{K}}, I S_{12}^{\mathcal{K}}, I S_{11}^{\kappa}, I S_{10}^{\kappa}$ we only have to consider the bottommost co-clones $I S_{1}^{2}, I S_{12}^{2}, I S_{11}^{2}, I S_{10}^{2}$. Now, observe that if $R$ and $R^{\prime}$ satisfies $R\left(x_{1}, \ldots, x_{\kappa}\right) \Rightarrow \exists y_{0}, y_{1} \cdot R^{\prime}\left(x_{1}, \ldots, x_{\kappa}, y_{0}, y_{1}\right) \wedge$ 
$F\left(y_{0}\right) \wedge T\left(y_{1}\right)$ and $R^{\prime}\left(x_{1}, \ldots, x_{\kappa}, y_{0}, y_{1}\right) \Rightarrow R\left(x_{1}, \ldots, x_{\kappa}\right) \wedge F\left(y_{0}\right)$, and it moreover holds that $R^{\prime}\left(x_{1}, \ldots, x_{\kappa}, y_{0}, y_{1}\right) \in\langle S\rangle_{\exists}$, then U-MAX-ONES $(\{R\}) \leq \mathrm{CV}$ U-MAX-ONES $(S)$, since we can use $y_{0}$ and $y_{1}$ as global variables, and because an optimal solution to the instance we construct will always map $y_{1}$ to 1 if the original instance is satisfiable. For $R_{\mathrm{IS}_{1}^{2}}\left(x_{1}, x_{2}, c_{0}\right)$ we can q.f.p.p. define predicates $R_{\mathrm{IS}_{1}^{2}}^{\prime}\left(x_{1}, x_{2}, c_{0}, y_{0}, y_{1}\right)$ with $R_{\mathrm{IS}_{12}^{2}}, R_{\mathrm{IS}_{11}^{2}}, R_{\mathrm{IS}_{10}^{2}}, R_{\mathrm{IE}_{2}}, R_{\mathrm{IE}_{0}}$ satisfying these properties as follows:

- $R_{\mathrm{IS}_{1}^{2}}^{\prime}\left(x_{1}, x_{2}, c_{0}, y_{0}, y_{1}\right) \equiv R_{\mathrm{IS}_{12}^{2}}\left(x_{1}, x_{2}, c_{0}, y_{1}\right) \wedge R_{\mathrm{IS}_{12}^{2}}\left(x_{1}, x_{2}, y_{0}, y_{1}\right)$,

- $R_{\mathrm{IS}_{1}^{2}}^{\prime}\left(x_{1}, x_{2}, c_{0}, y_{0}, y_{1}\right) \equiv R_{\mathrm{IS}_{11}^{2}}\left(x_{1}, x_{2}, y_{1}, c_{0}\right) \wedge R_{\mathrm{IS}_{11}^{2}}\left(x_{1}, x_{2}, y_{1}, y_{0}\right)$,

- $R_{\mathrm{IS}_{1}^{2}}^{\prime}\left(x_{1}, x_{2}, c_{0}, y_{0}, y_{1}\right) \equiv R_{\mathrm{IS}_{10}^{2}}\left(x_{1}, x_{2}, y_{1}, c_{0}, y_{1}\right) \wedge R_{\mathrm{IS}_{10}^{2}}\left(x_{1}, x_{2}, y_{1}, y_{0}, y_{1}\right)$,

- $R_{\mathrm{IS}_{1}^{2}}^{\prime}\left(x_{1}, x_{2}, c_{0}, y_{0}, y_{1}\right) \equiv R_{\mathrm{IE}_{2}}\left(c_{0}, x_{1}, x_{2}, c_{0}, y_{1}\right) \wedge R_{\mathrm{IE}_{2}}\left(c_{0}, x_{1}, x_{2}, y_{0}, y_{1}\right)$,

- $R_{\mathrm{IS}_{1}^{2}}^{\prime}\left(x_{1}, x_{2}, c_{0}, y_{0}, y_{1}\right) \equiv R_{\mathrm{IE}_{0}}\left(c_{0}, x_{1}, x_{2}, y_{1}, c_{0}\right) \wedge R_{\mathrm{IE}_{0}}\left(y_{0}, x_{1}, x_{2}, y_{1}, y_{0}\right)$,

and similarly a relation $R_{\mathrm{II}_{2}}^{\prime}$ using $R_{\mathrm{II}_{0}}$ as follows

$$
R_{\mathrm{II}_{2}}^{\prime}\left(x_{1}, x_{2}, x_{3}, x_{4}, x_{5}, x_{6}, c_{0}, c_{1}, y_{0}, y_{1}\right) \equiv \phi,
$$

where $\phi$ is the q.f.p.p. formula

$$
\begin{array}{r}
\phi \equiv R_{\mathrm{II}_{0}}\left(x_{1}, x_{2}, x_{3}, c_{0}\right) \wedge R_{\mathrm{II}_{0}}\left(c_{0}, c_{1}, y_{1}, y_{0}\right) \wedge \\
R_{\mathrm{II}_{0}}\left(x_{1}, x_{4}, y_{1}, y_{0}\right) \wedge R_{\mathrm{II}_{0}}\left(x_{2}, x_{5}, y_{1}, y_{0}\right) \wedge R_{\mathrm{II}_{0}}\left(x_{3}, x_{6}, y_{1}, y_{0}\right) .
\end{array}
$$

By Figure 2.1 in Chapter 2 we then see that the only remaining cases for $S$ when $\langle S\rangle \supset \mathrm{IS}_{1}^{2}$ is when $\langle S\rangle=\mathrm{II}_{2}$ or when $\langle S\rangle=\mathrm{ID}_{2}$. This concludes the proof.

Using q.f.p.p. implementations to further decrease the set of relations in Theorem 8.5 appears difficult and we therefore make use of more powerful implementations. Let $\operatorname{Optsol}(I)$ be the set of all optimal solutions of a W-MAX-Ones $(S)$ instance $I$. A relation $R$ has a weighted primitive positive definition (w.p.p. definition) [JKT11; Tha10] in $S$ if there exists an instance $I$ of W-MAX-ONES $(S)$ on variables $V$ such that

$$
R=\left\{\left(\phi\left(v_{1}\right), \ldots, \phi\left(v_{m}\right)\right) \mid \phi \in \operatorname{Optsol}(I)\right\}
$$

for some $v_{1}, \ldots, v_{m} \in V$. The set of all relations w.p.p. definable in $S$ is denoted $\langle S\rangle_{w}$ and we furthermore have that if $S$ and $S^{\prime}$ are finite constraint languages such that $S^{\prime} \subseteq\langle S\rangle_{w}$ then W-MAX-ONES $\left(S^{\prime}\right)$ is polynomial-time reducible to W-MAX-ONES $(S)$ [JKT11; Tha10]. If there is a W-MAX-ONES $(S)$ instance $I$ on $V$ such that

$$
R=\left\{\left(\phi\left(v_{1}\right), \ldots, \phi\left(v_{m}\right)\right) \mid \phi \in \operatorname{Optsol}(I)\right\}
$$

for $v_{1}, \ldots, v_{m} \in V$ satisfying $\left\{v_{1}, \ldots, v_{m}\right\}=V$, then we say that $R$ has a quantifier-free weighted primitive positive definition (q.f.w.p.p. definition) in $S$. 
We use $\langle S\rangle_{\nexists, w}$ for set of all relations q.f.w.p.p. definable in $S$. It is not hard to check that if $S^{\prime} \subseteq\langle S\rangle_{\nexists, w}$, then every instance is mapped to an instance of equally many variables - hence W-MAX-ONES $\left(S^{\prime}\right)$ is CV-reducible to $\mathrm{W}$-MAX-ONES $(S)$ whenever $S^{\prime}$ is finite. This gives the following corollary.

Corollary 8.6. Let $S$ and $S^{\prime}$ be finite constraint languages such that $S^{\prime} \subseteq\langle S\rangle_{\nexists, w}$. Then W-MAX-Ones $\left(S^{\prime}\right) \leq{ }^{\mathrm{CV}}$ W-MaX-Ones $(S)$.

By using q.f.w.p.p. definitions together with Theorem 8.5 we are now in a position to prove that W-MAX-ONES $\left(\left\{R_{\mathrm{II}_{2}}\right\}\right)$ is CV-reducible to any other NP-complete W-MAX-ONES $(S)$ problem.

Theorem 8.7. Let $S$ be a finite constraint language such that W-MAX-ONES $(S)$ is NP-complete. Then W-MAX-ONES $\left(\left\{R_{\mathrm{II}_{2}}\right\}\right) \leq \mathrm{CV}$ W-MAX-ONES $(S)$.

Proof. We need to prove that $R_{\mathrm{II}_{2}} \in\langle R\rangle_{\nexists, w}$ for every $R \in$ $\left\{R_{\mathrm{IS}_{1}^{2}}, R_{\mathrm{IN}_{2}}, R_{\mathrm{IL}_{0}}, R_{\mathrm{IL}_{2}}, R_{\mathrm{IL}_{3}}, R_{\mathrm{ID}_{2}}\right\}$. Let us first consider the case of $R_{\mathrm{II}_{2}} \in$ $\left\langle R_{\mathrm{IN}_{2}}\right\rangle_{\nexists, w}$. First, note that

$$
R_{\mathrm{II}_{2}}=\arg \max _{x \in \mathbb{B}^{8}:\left(x_{7}, x_{1}, x_{2}, x_{6}, x_{8}, x_{4}, x_{5}, x_{3}\right) \in R_{\mathrm{IN}_{2}}} x_{8},
$$

where $x$ is an abbreviation of $\left(x_{1}, x_{2}, x_{3}, x_{4}, x_{5}, x_{6}, x_{7}, x_{8}\right)$. We will see that it is not difficult to obtain a q.f.w.p.p. definition of $R_{\mathrm{II}_{2}}$ with $R_{\mathrm{IN}_{2}}$, armed with this information. Let $I$ be the W-MAX-ONES $\left(\left\{R_{I_{N}}\right\}\right)$ instance over the variables $\left\{x_{1}, \ldots, x_{8}\right\}$ with the single constraint $R_{\mathrm{IN}_{2}}\left(x_{7}, x_{1}, x_{2}, x_{6}, x_{8}, x_{4}, x_{5}, x_{3}\right)$, where $x_{8}$ have the weight 2 and all other variables the weight 1 . It is then easy to see that $I$ has three optimal solutions $\phi_{1}, \phi_{2}$, and $\phi_{3}$ such that

1. $\left(\left(\phi_{1}\left(x_{1}\right), \phi_{1}\left(x_{2}\right), \phi_{1}\left(x_{3}\right), \phi_{1}\left(x_{4}\right), \phi_{1}\left(x_{5}\right), \phi_{1}\left(x_{6}\right), \phi_{1}\left(x_{7}\right), \phi_{1}\left(x_{8}\right)\right)\right)=$ $(0,0,1,1,1,0,0,1) \in R_{\mathrm{II}_{2}}$,

2. $\left(\left(\phi_{2}\left(x_{1}\right), \phi_{2}\left(x_{2}\right), \phi_{2}\left(x_{3}\right), \phi_{2}\left(x_{4}\right), \phi_{2}\left(x_{5}\right), \phi_{2}\left(x_{6}\right), \phi_{2}\left(x_{7}\right), \phi_{2}\left(x_{8}\right)\right)\right)=$ $(0,1,0,1,0,1,0,1) \in R_{\mathrm{II}_{2}}$, and

3. $\left(\left(\phi_{3}\left(x_{1}\right), \phi_{3}\left(x_{2}\right), \phi_{3}\left(x_{3}\right), \phi_{3}\left(x_{4}\right), \phi_{3}\left(x_{5}\right), \phi_{3}\left(x_{6}\right), \phi_{3}\left(x_{7}\right), \phi_{3}\left(x_{8}\right)\right)\right)=$ $(1,0,0,0,1,1,0,1) \in R_{\mathrm{II}_{2}}$,

which implies that $R_{\mathrm{II}_{2}} \in\langle R\rangle_{\nexists, w}$. Hence, it is rather easy, albeit tedious, to prove that a relation is q.f.w.p.p. definable over another relation when given an equality akin to ( $\oplus$. To avoid boring the reader more than necessary, we have prepared these arg max definitions in Table 8.1. from which it is easy to derive the necessary q.f.w.p.p. definitions.

The Argus-eyed reader might notice a slight discrepancy between the problem W-MAX-ONES $\left(\left\{R_{I_{2}}\right\}\right)$ and the problem $\operatorname{SAT}\left(\left\{R_{1 / 3}^{\neq \neq \neq}\right\}\right)$from Chapter 7 In this chapter we have chosen to work with $R_{\mathrm{II}_{2}}=R_{1 / 3}^{\neq \neq \neq 01}$ rather than with $R_{1 / 3}^{\neq \neq \neq}$since it simplifies some of the proofs. It is however easy to see that W-MAX-ONES $\left(\left\{R_{1 / 3}^{\neq \neq \neq}\right\}\right) \leq \mathrm{CV}$ W-MAX-ONES $\left(\left\{R_{\mathrm{II}_{2}}\right\}\right)$ - hence, all the results in this Chapter stated for W-MAX-ONES $\left(\left\{R_{\mathrm{II}_{2}}\right\}\right)$ could equivalently well be stated for W-MAX-ONES $\left(\left\{R_{1 / 3}^{\neq \neq \neq}\right\}\right)$. 
Table 8.1: arg max definitions for W-MAX-ONES $(\cdot)$.

\begin{tabular}{|c|c|c|}
\hline$R_{1}$ & $R_{2}$ & arg max definition of $R_{1}$ with $R_{2}$ \\
\hline$R_{\mathrm{II}_{2}}$ & $R_{\mathrm{IN}_{2}}$ & $\arg \max _{x \in \mathbb{B}^{8}:\left(x_{7}, x_{1}, x_{2}, x_{6}, x_{8}, x_{4}, x_{5}, x_{3}\right) \in R_{I N}} x_{8}$ \\
\hline$R_{\mathrm{II}_{2}}$ & $R_{\mathrm{ID}_{2}}$ & 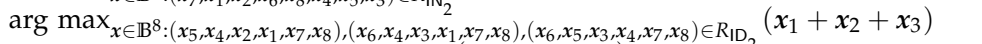 \\
\hline$R_{\mathrm{II}_{2}}$ & $R_{\mathrm{IL}_{2}}$ & $\arg \max _{x \in \mathbb{B}^{8}:\left(x_{4}, x_{5}, x_{6}, x_{1}, x_{2}, x_{3}, x_{7}, x_{8}\right) \in R_{\mathrm{IL}}}\left(x_{4}+x_{5}+x_{6}\right)$ \\
\hline$R_{\mathrm{IL}_{2}}$ & $R_{\mathrm{IL}_{3}}$ & 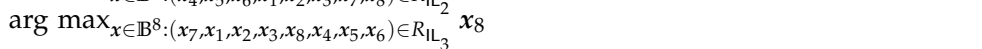 \\
\hline$R_{\mathrm{IL}_{2}}$ & $R_{\mathrm{IL}_{0}}$ & 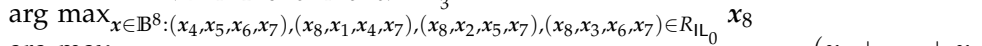 \\
\hline$R_{I_{2}}$ & $R_{\mathrm{IS}_{1}^{2}}^{2}$ & $\arg \max _{x \in \mathbb{B}^{8}:\left(x_{1}, x_{2}, x_{7}\right),\left(x_{1}, x_{3}, x_{7}\right),\left(x_{2}, x_{3}, x_{7}\right),\left(x_{1}, x_{4}, x_{7}\right),\left(x_{2}, x_{5}, x_{7}\right),\left(x_{3}, x_{6}, x_{7}\right) \in R_{\mathrm{IS}}^{2}}\left(x_{1}+\cdots+x_{8}\right.$ \\
\hline
\end{tabular}

\subsubsection{The VCSP Problem}

Since the $\operatorname{VCSP}(\cdot)$ problem does not adhere to the standard Galois connection in Theorem 2.11. the weak base method is not applicable and alternative methods are required. For this purpose we use multimorphisms from Cohen et al. [CCJK06]. Let $\Delta$ be a set of cost functions on $\mathbb{B}$, let $p$ be a unary operation on $\mathbb{B}$, and let $f, g$ be binary operations on $\mathbb{B}$. We say that $\Delta$ admits the binary multimorphism $(f, g)$ if it holds that $v(f(x, y))+v(g(x, y)) \leq v(x)+v(y)$ for every $v \in \Delta$ and $x, y \in \mathbb{B}^{\operatorname{ar}(v)}$. Similarly, $\Delta$ admits the unary multimorphism $(p)$ if it holds that $v(p(x)) \leq v(x)$ for every $v \in \Delta$ and $x \in \mathbb{B}^{\operatorname{ar}(v)}$.

Recall that the function $f_{\neq}$is defined as $f_{\neq}(0,0)=f_{\neq}(1,1)=1$ and $f_{\neq}(0,1)=f_{\neq}(1,0)=0$. It is not difficult to see that the minimisation problem $\operatorname{VCSP}\left(\left\{f_{\neq}\right\}\right)$and the maximisation problem MAx CUT are trivially $\mathrm{CV}$-reducible to each other. We will make use of (a variant of) the concept of expressibility [CCJK06] to prove that $\operatorname{VCSP}\left(\left\{f_{\neq}\right\}\right)$is CV-reducible to any other Boolean, NP-complete $\operatorname{VCSP}(\Delta)$ problem. Say that a cost function $g$ is \#-expressible in $\Delta$ if $g\left(x_{1}, \ldots, x_{n}\right)=\sum_{i} w_{i} f_{i}\left(s_{i}\right)+w$ for some $\operatorname{ar}\left(f_{i}\right)$-ary tuples $s_{i}$ over $\left\{x_{1}, \ldots, x_{n}\right\}$, weights $w_{i} \in \mathbb{Q}_{\geq 0}, w \in \mathbb{Q}$ and $f_{i} \in \Delta$. It is not hard to see that if every function in a finite set $\Delta^{\prime}$ is $\nexists$-expressible in $\Delta$, then $\operatorname{VCSP}\left(\Delta^{\prime}\right) \leq \operatorname{CV} \operatorname{VCSP}(\Delta)$. Note that if the constants 0 and 1 are expressible in $\Delta$ then we may allow tuples $s_{i}$ over $\left\{x_{1}, \ldots, x_{n}, 0,1\right\}$, and still obtain a CV-reduction.

Theorem 8.8. Let $\Delta$ be a set of finite-valued cost functions on $\mathbb{B}$. If $\operatorname{VCSP}(\Delta)$ is $N P$-complete, then $\operatorname{VCSP}\left(\left\{f_{\neq}\right\}\right) \leq \mathrm{CV} \operatorname{VCSP}(\Delta)$.

Proof. The proof consists of two parts. Using the assumption that $\operatorname{VCSP}(\Delta)$ is NP-complete we will (1) prove that we can force two variables $v_{0}$ and $v_{1}$ to 0 and 1 , respectively, and (2) show that there exists a certain function which is $\nexists$-expressible over $\Delta$, which together with the two constant variables $v_{0}$ and $v_{1}$ can express $f_{\neq}$.

First, since $\operatorname{VCSP}(\Delta)$ is $\mathrm{NP}$-complete (and since we assume $\mathrm{P} \neq \mathrm{NP}$ ) we know that $\Delta$ does not admit the unary (0)-multimorphism or the unary (1)-multimorphism [CCJK06]. Therefore there are $g, h \in \Delta$ and $\boldsymbol{u} \in \mathbb{B}^{\operatorname{ar}(g)}, \boldsymbol{v} \in \mathbb{B}^{\operatorname{ar}(h)}$ such that $g(\mathbf{0})>g(\boldsymbol{u})$ and $h(\mathbf{1})>h(\boldsymbol{v})$. Let $\boldsymbol{w} \in \arg \min _{x \in \mathbb{B}^{b}}\left(g\left(x_{1}, \ldots, x_{a}\right)+h\left(x_{a+1}, \ldots, x_{b}\right)\right)$ and then define $o(x, y)=$ $g\left(z_{1}, \ldots, z_{a}\right)+h\left(z_{a+1}, \ldots, z_{b}\right)$ where $z_{i}=x$ if $\boldsymbol{w}_{i}=0$ and $z_{i}=y$ otherwise. 
Clearly $(0,1) \in \arg \min _{x \in \mathbb{B}^{2}} o(\boldsymbol{x}), o(0,1)<o(0,0)$, and $o(0,1)<o(1,1)$. We will show that we always can force two fresh variables $v_{0}$ and $v_{1}$ to 0 and 1 , respectively. If $o(0,0) \neq o(1,1)$, then assume without loss of generality that $o(0,0)<o(1,1)$. In this case we force $v_{0}$ to 0 with the (sufficiently weighted) term $o\left(v_{0}, v_{0}\right)$. Define $g^{\prime}(x)=g\left(z_{1}, \ldots, z_{\operatorname{ar}(g)}\right)$ where $z_{i}=x$ if $u_{i}=1$ and $z_{i}=v_{0}$ otherwise. Note that $g^{\prime}(1)<g^{\prime}(0)$ which means that we can force $v_{1}$ to 1 . Otherwise $o(0,0)=o(1,1)$. If $o(0,1)=o(1,0)$, then $f_{\neq}=\alpha_{1} o+\alpha_{2}$, otherwise assume without loss of generality that $o(0,1)<o(1,0)$. In this case $v_{0}, v_{1}$ can be forced to 0,1 with the help of the (sufficiently weighted) term $o\left(v_{0}, v_{1}\right)$.

Second, we also know that $\Delta$ does not admit the (min,max)multimorphism [CCJK06] since $\operatorname{VCSP}(\Delta)$ is NP-hard by assumption. Hence, there exists an $n$-ary function $f \in \Delta$ and $s, t \in \mathbb{B}^{n}$ such that $f(\min (s, t))+$ $f(\max (s, t))>f(s)+f(\boldsymbol{t})$. Let $f_{1}(x)=\alpha_{1} o\left(v_{0}, x\right)+\alpha_{2}$ for some $\alpha_{1} \in \mathbb{Q}_{\geq 0}$ and $\alpha_{2} \in \mathbb{Q}$ such that $f_{1}(1)=0$ and $f_{1}(0)=1$. Also let $g(x, y)=f\left(z_{1}, \ldots, z_{n}\right)$ where $z_{i}=v_{1}$ if $\min \left(\boldsymbol{s}_{i}, \boldsymbol{t}_{i}\right)=1, z_{i}=v_{0}$ if $\max \left(\boldsymbol{s}_{i}, \boldsymbol{t}_{i}\right)=0, z_{i}=x$ if $s_{i}>t_{i}$ and $z_{i}=y$ otherwise. Note that $g(0,0)=f(\min (s, t)), g(1,1)=f(\max (s, t))$, $g(1,0)=f(s)$ and $g(0,1)=f(\boldsymbol{t})$. Set $h(x, y)=g(x, y)+g(y, x)$. Now $h(0,1)=h(1,0)<\frac{1}{2}(h(0,0)+h(1,1))$. If $h(0,0)=h(1,1)$, then $f_{\neq}=$ $\alpha_{1} h+\alpha_{2}$ for some $\alpha_{1} \in \mathbb{Q}_{\geq 0}$ and $\alpha_{2} \in \mathbb{Q}$. Hence, we can without loss of generality assume that $h(1,1)-h(0,0)=2$. Note now that $h^{\prime}(x, y)=$ $f_{1}(x)+f_{1}(y)+h(x, y)$ satisfies $h^{\prime}(0,0)=h^{\prime}(1,1)=\frac{1}{2}(h(0,0)+h(1,1)+2)$ and $h^{\prime}(0,1)=h^{\prime}(1,0)=\frac{1}{2}(2+h(0,1)+h(1,0))$. Hence, $h^{\prime}(0,0)=h^{\prime}(1,1)>$ $h^{\prime}(0,1)=h^{\prime}(1,0)$. So $f_{\neq}=\alpha_{1} h^{\prime}+\alpha_{2}$ for some $\alpha_{1} \in \mathbb{Q}_{\geq 0}$ and $\alpha_{2} \in \mathbb{Q}$.

\subsubsection{The Broader Picture}

Theorems 8.7 and 8.8 do not describe the relative complexity between the $\operatorname{SAT}(\cdot), \operatorname{MAX}-\mathrm{ONES}(\cdot)$ and $\operatorname{VCSP}(\cdot)$ problems. However, we readily see (1) that $\operatorname{SAT}\left(\left\{R_{\mathrm{II}_{2}}\right\}\right) \leq \mathrm{CV}$ W-MAX-ONES $\left(\left\{R_{\mathrm{II}_{2}}\right\}\right)$, and (2) that W-MAX-ONES $\left(\left\{R_{\mathrm{II}_{2}}\right\}\right) \leq \mathrm{CV}_{\mathrm{W}}$-MAX INDEPENDENT SET since W-MAX INDEPENDENT SET can be expressed by W-MAX-ONES $\left(\left\{\mathrm{NAND}^{2}\right\}\right)$. The problem W-MAX-ONES $\left(\left\{\right.\right.$ NAND $\left.\left.^{2}\right\}\right)$ is in turn expressible by MAX-CSP $\left(\left\{\mathrm{NAND}^{2}\right.\right.$, $\mathrm{T}, \mathrm{F}\})$. To show that W-MAX INDEPENDENT $\operatorname{SET} \leq \mathrm{CV} \operatorname{VCSP}\left(\left\{f_{\neq}\right\}\right)$it is in fact, since $\operatorname{MAX}-\operatorname{CSP}(\{\mathrm{Neq}\})$ and $\operatorname{VCSP}\left(\left\{f_{\neq}\right\}\right)$are $\operatorname{CV}$-reducible to each other, sufficient to show that $\operatorname{MAX}-\operatorname{CSP}\left(\left\{\mathrm{NAND}^{2}, \mathrm{~T}, \mathrm{~F}\right\}\right) \leq \mathrm{CV}$ $\operatorname{MAX}-\operatorname{CSP}(\{\mathrm{Neq}\})$. We do this as follows. Let $v_{0}$ and $v_{1}$ be two global variables. We force $v_{0}$ and $v_{1}$ to be mapped to different values by assigning a sufficiently high weight to the constraint $\operatorname{Neq}\left(v_{0}, v_{1}\right)$. It then follows that $\mathrm{T}(x)=\operatorname{Neq}\left(x, v_{0}\right), \mathrm{F}(x)=\operatorname{Neq}\left(x, v_{1}\right)$ and $\operatorname{NAND}^{2}(x, y)=$ $\frac{1}{2}(\operatorname{Neq}(x, y)+\mathrm{F}(x)+\mathrm{F}(y))$, and we are done. It follows from this proof that $\operatorname{MAX}-\mathrm{CSP}\left(\left\{\mathrm{NAND}^{2}, \mathrm{~T}, \mathrm{~F}\right\}\right)$ and $\operatorname{VCSP}\left(\left\{f_{\neq}\right\}\right)$are mutually CVinterreducible. Since $\operatorname{MAX}-\mathrm{CSP}\left(\left\{\mathrm{NAND}^{2}, \mathrm{~T}, \mathrm{~F}\right\}\right)$ can also be formulated as a $\operatorname{VCSP}(\cdot)$ problem, it follows that $\operatorname{VCSP}(\cdot)$ does not have a unique easiest set of cost functions. The complexity results are summarised in Figure 8.1. 


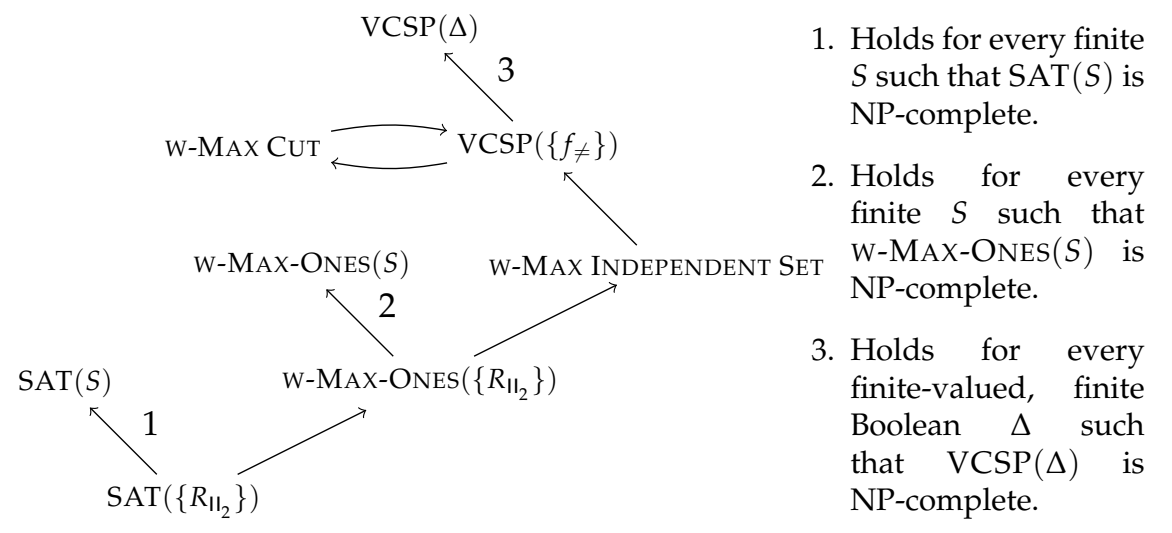

Figure 8.1: The complexity landscape of some Boolean optimisation and satisfiability problems. A directed arrow from one node $A$ to $B$ means that $A \leq \mathrm{CV} B$.

Some trivial inclusions are omitted in the figure: for example it holds that $\operatorname{SAT}(S) \leq{ }^{\mathrm{CV}}$ W-MAX-ONES $(S)$ for all $S$.

\subsection{Subexponential Time and the Exponential-Time Hypothesis}

In this section we investigate the consequences of the ETH for the U-MAX-ONES and U-VCSP problems. A direct consequence of Section 8.3 is that if there exists any finite constraint language $S$ or set of cost functions $\Delta$ such that W-MAx-ONES $(S)$ or $\operatorname{VCSP}(\Delta)$ is NP-complete and in SE, then $\operatorname{SAT}\left(\left\{R_{\mathrm{II}_{2}}\right\}\right)$ is in SE which according to Theorem 7.40 in Chapter 7implies that the ETH is false. For the unweighted MAX-ONES problem we can however make these results even more precise, and prove that the ETH is true if and only if there does not exist any finite constraint language $S$ such that U-MAX-ONES $(S)$ is NP-complete and solvable in subexponential time. Before we begin we prove one property of the problem SAT $\left(\left\{R_{\mathrm{II}_{2}}\right\}\right)$-DEG-2 that will be needed later on.

Lemma 8.9. $\operatorname{SAT}\left(\left\{R_{\mathrm{II}_{2}}\right\}\right)-D E G-2 \in \mathrm{SE}$ if and only if $\operatorname{SAT}\left(\left\{R_{1 / 3}^{\neq \neq \neq}\right\}\right)-D E G-2 \in$ SE.

Proof. First, assume that $\operatorname{SAT}\left(\left\{R_{\mathrm{II}_{2}}\right\}\right)$-DEG-2 $\in$ SE, and let $I=(V, C)$ be an instance of $\operatorname{SAT}\left(\left\{R_{1 / 3}^{\neq \neq \neq}\right\}\right)$-DEG-2. For each constraint $C_{i}=$ $R_{1 / 3}^{\neq \neq \neq}\left(x_{i_{1}}, x_{i_{2}}, x_{i_{3}}, x_{i_{4}}, x_{i_{5}}, x_{i_{6}}\right)$, introduce two fresh variables $y_{i}$ and $y_{i}^{\prime}$ and replace $C_{i}$ by the constraint $R_{\mathrm{II}_{2}}\left(x_{i_{1}}, x_{i_{2}}, x_{i_{3}}, x_{i_{4}}, x_{i_{5}}, x_{i_{6}}, y_{i}, y_{i}^{\prime}\right)$. Let $\left(V^{\prime}, C^{\prime}\right)$ be the resulting instance. Since $|C| \leq 2|V|$, and since we for each constraint introduce exactly two fresh variables, it follows that $\left|V^{\prime}\right| \leq 4|V|$ and that the reduction is an LV-reduction. 
Second, assume that $\operatorname{SAT}\left(\left\{R_{1 / 3}^{\neq \neq \neq}\right\}\right)$-DEG-2 $\in$ SE, and observe that, by Theorem 7.40 in Chapter 7, also implies that $\operatorname{SAT}\left(\left\{R_{\mathrm{II}_{2}}\right\}\right) \in \mathrm{SE}$. Hence, $\operatorname{SAT}\left(\left\{R_{\mathrm{II}_{2}}\right\}\right)-\mathrm{DEG}-2$ is trivially in SE, too.

Lemma 8.10. If U-MAX-ONES $(S)$ is in SE for some finite constraint language $S$ such that U-MAX-ONES $(S)$ is NP-complete, then the ETH is false.

Proof. From Theorem 7.40 in Chapter 7 it follows that 3-SAT is in SE if and only if $\operatorname{SAT}\left(\left\{R_{1 / 3}^{\neq \neq \neq}\right\}\right)-\mathrm{DEG}-2$ is in SE. By Lemma 8.9 it furthermore follows that $\operatorname{SAT}\left(\left\{R_{\mathrm{II}_{2}}\right\}\right)$-DEG-2 $\in \mathrm{SE}$. Combining this with Theorem 8.5 we only have to prove that $\operatorname{SAT}\left(\left\{R_{\mathrm{II}_{2}}\right\}\right)-2 \mathrm{LV}$-reduces to U-MAX-ONES $(\{R\})$ for $R \in$ $\left\{R_{\mathrm{IS}_{1}^{2}}, R_{\mathrm{IIN}_{2}}, R_{\mathrm{IL}_{0}}, R_{\mathrm{IL}_{2}}, R_{\mathrm{IL}_{3}}, R_{\mathrm{ID}_{2}}\right\}$. We provide an illustrative reduction from $\operatorname{SAT}\left(\left\{R_{\mathrm{II}_{2}}\right\}\right)$-DEG-2 to U-MAX-ONES $\left(\left\{R_{\mathrm{IS}_{1}^{2}}\right\}\right)$; the remaining reductions are presented in Lemmas 8.148 .18 in Section 8.5 Since $R_{\mathrm{IS}_{1}^{2}}$ is the NAND relation with one additional constant column, the U-MAX-ONES $\left(\left\{R_{\mathrm{IS}_{1}^{2}}\right)\right\}$ problem is basically the maximum independent set problem or, equivalently, the maximum clique problem in the complement graph. Given an instance $I$ of $\operatorname{CSP}\left(\left\{R_{\mathrm{II}_{2}}\right\}\right)$-DEG-2 we create for every constraint 3 vertices, one corresponding to each feasible assignment of values to the variables occurring in the constraint. We add edges between all pairs of vertices that are not inconsistent and that do not correspond to the same constraint. The instance $I$ is satisfied if and only if there is a clique of size $m$ where $m$ is the number of constraints in $I$. Since $m \leq 2|\operatorname{Var}(I)|$ this implies that the number of vertices is $\leq 2|\operatorname{Var}(I)|$.

Hence, we have ruled out the possibility that, assuming the ETH, there could exist a U-MAX-ONES $(S) \in \mathrm{SE}$ problem which is NP-complete. It is also not difficult to prove the opposite, i.e., that if the ETH is false, then $\mathrm{U}-\mathrm{MAX}-\mathrm{OnES}(S) \in \mathrm{SE}$ ) for every finite Boolean constraint language $S$.

Lemma 8.11. If the ETH is false, then $\mathrm{U}-\mathrm{MAX}-\mathrm{ONES}(S) \in \mathrm{SE}$ for every finite Boolean constraint language $S$.

Proof. Define SNP to be the class of properties expressible by formulas of the type $\exists S_{1} \ldots \exists S_{n} \forall x_{1} \ldots \forall x_{m} . F$ where $F$ is a quantifier-free logical formula, $\exists S_{1} \ldots \exists S_{n}$ are second order existential quantifiers, and $\forall x_{1} \ldots \forall x_{m}$ are firstorder universal quantifiers. Monadic SNP (MSNP) is the restriction of SNP where all second-order predicates are required to be unary [FV98]. The associated search problem tries to identify instantiations of $S_{1}, \ldots, S_{n}$ that make the resulting first-order formula true. We will be interested in properties that can be expressed by formulas that additionally contain size-constrained existential quantifier. A size-constrained existential quantifier is of the form $\exists S,|S| \oplus S$, where $|S|$ is the number of inputs where relation $S$ holds, and $\oplus \in\{=, \leq, \geq\}$. Define size-constrained SNP as the class of properties of relations and numbers that are expressible by formulas $\exists S_{1} \ldots \exists S_{n} \forall x_{1} \ldots \forall x_{m} . F$ where the existential quantifiers are allowed to be size-constrained. 
If the ETH is false then 3-SAT is solvable in subexponential time. By Impagliazzo et al. [IP01] this problem is size-constrained MSNP-complete under size-preserving SERF reductions. Hence, we only have to prove that U-MAX-ONES $(\cdot)$ is included in size-constrained MSNP for it to be solvable in subexponential time. Impagliazzo et al. |IP01| shows that $\kappa$-SAT is in SNP by providing an explicit formula $\exists S$.F where $F$ is a universal formula and $S$ a unary predicate interpreted such that $x \in S$ if and only if $x$ is true. Let $\kappa$ be the highest arity of any relation in $S$. Since $\kappa$-SAT can q.f.p.p. implement any $\kappa$-ary Boolean relation it is therefore sufficient to prove that $\mathrm{U}-\mathrm{MAX}-\mathrm{ONES}\left(\Gamma_{\mathrm{SAT}}^{\kappa}\right)$ is in size-constrained MSNP. This is easy to do with the formula

$$
\exists S,|S| \geq K . F
$$

where $K$ is the parameter corresponding to the number of variables that have to be assigned 1 .

To obtain similar results for $\operatorname{VCSP}(\cdot)$, we have to impose additional restrictions, and only consider the problem $\mathrm{U}-\mathrm{VCSP}_{d}(\cdot)$ (recall from Section 8.2 that $\mathrm{U}-\mathrm{VCSP}_{d}(\Delta)$ denotes the unweighted $\operatorname{VCSP}(\Delta)$ problem where each instance $I$ contains at most $d|\operatorname{Var}(I)|$ constraints).

Lemma 8.12. If $\mathrm{U}-\mathrm{MAX}-\mathrm{ONES}(S) \in \mathrm{SE}$ for every finite Boolean constraint language $S$, then $\mathrm{U}-\mathrm{VCSP}_{d}(\Delta) \in \mathrm{SE}$ for every finite set of Boolean cost functions $\Delta$ and arbitrary $d \geq 0$.

Proof. We first show that if every U-MAX-ONES(S) $\in$ SE, then $\mathrm{U}-\mathrm{Min}-\mathrm{OnES}(S) \in \mathrm{SE}$ for all $S$, too. Here U-Min-OnES $(S)$ denotes the minimisation variant of U-MAX-ONES $(S)$ where the goal instead is to minimise the number of variables assigned 1. Arbitrarily choose a finite constraint language $S$ over $\mathbb{B}$. We present an LV-reduction from U-MIN-ONES $(S)$ to U-MAX-ONES $(S \cup\{\mathrm{Neq}\})$. Let $\left(\left\{v_{1}, \ldots, v_{n}\right\}, C\right)$ be an arbitrary instance of U-Min-OneS $(S)$ with optimal value $K$. Consider the instance $I=\left(V^{\prime}, C^{\prime}\right)$ of $\mathrm{U}-\mathrm{MAX}-\mathrm{ONES}(S \cup\{\mathrm{Neq}\})$ where:

- $V^{\prime}=\left\{v_{1}, v_{1}^{\prime}, v_{1}^{\prime \prime}, \ldots, v_{n}, v_{n}^{\prime}, v_{n}^{\prime \prime}\right\}$, and

- $C^{\prime}=C \cup\left\{\operatorname{Neq}\left(v_{1}, v_{1}^{\prime}\right), \operatorname{Neq}\left(v_{1}, v_{1}^{\prime \prime}\right), \ldots, \operatorname{Neq}\left(v_{n}, v_{n}^{\prime}\right), \operatorname{Neq}\left(v_{n}, v_{n}^{\prime \prime}\right\}\right.$.

For each variable $v_{i} \in\left\{v_{1}, \ldots, v_{n}\right\}$ that is assigned 0 , the corresponding variables $v_{i}^{\prime}, v_{i}^{\prime \prime}$ are assigned 1 , and vice-versa. It follows that the optimal value of $I^{\prime}$ is $2 n-K$. Hence, U-MIN-OnES $(S) \in$ SE since U-MAX-ONES $(S \cup$ $\{\mathrm{Neq}\}) \in \mathrm{SE}$.

Now, arbitrarily choose $d \geq 0$ and a finite set of Boolean cost functions $\Delta$. Since $\Delta$ is finite, we may without loss of generality assume that each function $f \in \Delta$ has its range in $\{0,1,2, \ldots\}$.

We show that $\mathrm{U}-\operatorname{VCSP}_{d}(\Delta) \in \mathrm{SE}$ by exhibiting an LV-reduction from $\mathrm{U}-\operatorname{VCSP}_{d}(\Delta)$ to U-MIN-ONES $(S)$ where $S$ is finite and only depends on $\Delta$. 
Given a tuple $\boldsymbol{a}=\left(a_{1}, \ldots, a_{k}\right) \in \mathbb{B}^{k}$, let $\operatorname{val}(\boldsymbol{a})=1+\sum_{j: a_{j}=1} 2^{j-1}$. For each $f \in \Delta$ of arity $k$, define

$$
\begin{aligned}
R_{f}= & \left\{\begin{array}{l|l}
\left.\left(x_{1}, \ldots, x_{k}, y_{1}, \ldots, y_{2^{k}}\right) \in \mathbb{B}^{k+2^{k}} \mid \begin{array}{l}
f\left(x_{1}, \ldots, x_{k}\right)>0, \\
\left\{i: y_{i} \neq 0\right\}=\left\{\operatorname{val}\left(x_{1}, \ldots, x_{k}\right)\right\}
\end{array}\right\} \\
\cup\left\{\left(x_{1}, \ldots, x_{k}, 0, \ldots, 0\right) \in \mathbb{B}^{k+2^{k}} \mid f\left(x_{1}, \ldots, x_{k}\right)=0\right\},
\end{array}\right.
\end{aligned}
$$

and let $S=\{\mathrm{Eq}, \mathrm{Neq}\} \cup\left\{R_{f} \mid f \in \Delta\right\}$.

One may interpret $R_{f}$ as follows: for each $\left(x_{1}, \ldots, x_{k}\right) \in \mathbb{B}^{k}$ the relation $R_{f}$ contains exactly one tuple $\left(x_{1}, \ldots, x_{k}, y_{1}, \ldots, y_{2^{k}}\right)$. If $f\left(x_{1}, \ldots, x_{k}\right)=0$, then this is the tuple $\left(x_{1}, \ldots, x_{k}, 0, \ldots, 0\right)$. If $f\left(x_{1}, \ldots, x_{k}\right)>0$, then this is the tuple $\left(x_{1}, \ldots, x_{k}, 0, \ldots, 1, \ldots, 0\right)$ where the 1 is in position $k+\operatorname{val}\left(x_{1}, \ldots, x_{k}\right)$. We show below how $R_{f}$ can be used for 'translating' each $x \in \mathbb{B}^{k}$ into its corresponding weight as prescribed by $f$.

Let $\left(V, \sum_{i=1}^{m} f_{i}\left(x_{i}\right)\right)$ be an arbitrary instance of $\mathrm{U}-\mathrm{VCSP}_{d}(\Delta)$ where $V=$ $\left\{v_{1}, \ldots, v_{n}\right\}$. Assume the instance has an optimal solution with value $K$. For each term $f_{i}\left(v_{1}, \ldots, v_{k}\right)$ in the sum, do the following:

1. introduce $2^{k}$ fresh variables $v_{1}^{\prime}, \ldots, v_{2^{k}}^{\prime}$

2. introduce $k$ fresh variables $w_{1}, \ldots, w_{k}$,

3. for each $\boldsymbol{a} \in \mathbb{B}^{k}$ such that $f_{i}(\boldsymbol{a})>1$, introduce $n^{\prime}=f_{i}(\boldsymbol{a})$ fresh variables $u_{0}, \ldots, u_{n^{\prime}-1}$

4. introduce the constraint $R_{f_{i}}\left(v_{1}, \ldots, v_{k}, v_{1}^{\prime}, \ldots, v_{2^{k}}^{\prime}\right)$ ，

5. introduce the constraints $\operatorname{Neq}\left(v_{1}, w_{1}\right), \ldots, \operatorname{Neq}\left(v_{k}, w_{k}\right)$, and

6. for each $\boldsymbol{a} \in \mathbb{B}^{k}$, let $n^{\prime}=f_{i}(\boldsymbol{a})$ and do the following if $n^{\prime}>1$ : let $p=\operatorname{val}(\boldsymbol{a})$ and introduce the constraints $\operatorname{Eq}\left(v_{p}^{\prime}, u_{0}\right), \operatorname{Eq}\left(u_{0}, u_{1}\right), \ldots, \operatorname{Eq}\left(u_{n^{\prime}-2}, u_{n^{\prime}-1}\right)$.

It is not difficult to realise that the resulting instance has optimal value $K+\sum_{i=1}^{m} \operatorname{ar}\left(f_{i}\right)$ given the interpretation of $R_{f}$ and the following motivation of step 5: the Neq constraints introduced in step 5 ensure that the weight of $\left(x_{1}, \ldots, x_{k}\right)$ does not influence the weight of the construction and this explains that we need to adjust the optimal value with $\sum_{i=1}^{m} \operatorname{ar}\left(f_{i}\right)$.

Furthermore, the instance contains at most

$$
|V|+|C| \cdot\left(2 s+t \cdot\left(2^{s}+1\right)\right)
$$

variables where $s=\max \{\operatorname{ar}(f) \mid f \in \Delta\}$ and $t=\max \{f(\boldsymbol{a}) \mid f \in$ $\Delta$ and $\left.a \in \mathbb{B}^{\operatorname{ar}(f)}\right\}$. By noting that $|C| \leq d|V|$ and that $s, t$ are constants that only depend on $\Delta$, it follows that the reduction is an LV-reduction.

Combining Lemmas 8.10, 8.11, and 8.12 we therefore obtain the following theorem. 
Theorem 8.13. The following statements are equivalent.

1. The exponential-time hypothesis is false.

2. U-MAX-OnES $(S) \in \operatorname{SE}$ for every finite $S$.

3. U-MAX-OnES $(S) \in \mathrm{SE}$ for some finite $S$ such that U-MAX-ONES $(S)$ is NP-complete.

4. $\mathrm{U}-\operatorname{VCSP}(\Delta)_{d} \in \mathrm{SE}$ for every finite set of finite-valued cost functions $\Delta$ and $d \geq 0$.

Proof. The implication $1, \Rightarrow 2$ follows from Lemma $8.11,2 \Rightarrow 3$ is trivial, and $3 \Rightarrow 1$ follows by Lemma 8.10 . The implication $2 \Rightarrow 4$ follows from Lemma 8.12 We finish the proof by showing $4 \Rightarrow 1$. Let $I=(V, C)$ be an instance of $\operatorname{SAT}\left(\left\{R_{\mathrm{II}_{2}}\right\}\right)$-DEG-2. Note that $I$ contains at most $2|V|$ constraints. Let $f$ be the function defined by $f(x)=0$ if $x \in R_{\mathrm{II}_{2}}$ and $f(x)=1$ otherwise. Create an instance of $\mathrm{U}-\operatorname{VCSP}_{2}(\{f\})$ by, for every constraint $C_{i}=R_{\mathrm{II}_{2}}\left(x_{1}, \ldots, x_{8}\right) \in C$, adding to the cost function the term $f\left(x_{1}, \ldots, x_{8}\right)$. This instance has a solution with objective value 0 if and only if $I$ is satisfiable. Hence, $\operatorname{SAT}\left(\left\{R_{\mathrm{II}_{2}}\right\}\right)$-DEG-2 $\in$ SE which contradicts the ETH according to Lemma 8.9 and Theorem 7.40 in Chapter 7 .

\subsection{Summary and Conclusions}

In this chapter we have proved that the existence of an 'easiest problem' is not a unique feature of SAT $(\cdot)$, and that our approach based on partial clone theory extends naturally to other problems, as long as they follow the standard Galois connection. Interestingly, the proof of Theorem 8.8, also shows that partial clone theory is not the only possible method for obtaining such results. Hence, if a problem follows the standard Galois connection and is parameterized with a Boolean constraint language, then weak bases coupled with CV-reductions provides a straightforward framework for proving the existence of an easiest problem; but even if this is not the case, there might exist other methods for proving analogous results.

\section{Additional Proofs for Section 8.4}

In this section we include the missing proofs of Lemma 8.10 in Section 8.4

Lemma 8.14. $\operatorname{SAT}\left(\left\{R_{\mathrm{II}_{2}}\right\}\right)-2 L V$-reduces to U-MAX-ONES $\left(\left\{R_{\mathrm{IL}_{2}}\right\}\right)$.

Proof. We reduce an instance $I=(V, C)$ of $\operatorname{SAT}\left(\left\{R_{\mathrm{II}_{2}}\right\}\right)-2$ to an instance of U-MAX-ONES $\left(\left\{R_{\mathrm{IL}_{2}}\right\}\right)$ containing at most $2+8|V|$ variables. Let $v_{0}, v_{1}$ be two fresh global variables and introduce the constraint $R_{\mathrm{IL}_{2}}\left(v_{0}, v_{0}, v_{0}, v_{1}, v_{1}, v_{1}, v_{0}, v_{1}\right)$. Note that this forces $v_{0}$ to 0 and $v_{1}$ to 1 in any satisfying assignment. Now, for every variable $x \in V$ we create an additional variable $x^{\prime}$ which we constrain as $R_{\mathrm{IL}_{2}}\left(x^{\prime}, x, v_{1}, x, x^{\prime}, v_{0}, v_{0}, v_{1}\right)$. This 
correctly implements $\mathrm{Neq}\left(x, x^{\prime}\right)$. Let $R_{\mathrm{II}_{2}}\left(x_{i}^{1}, \ldots, x_{i}^{6}, c_{0}, c_{1}\right)$ be a constraint in $C$. We introduce three fresh variables $z_{i}^{1}, z_{i}^{2}, z_{i}^{3}$ and constrain them as $R_{\mathrm{IL}_{2}}\left(z_{i}^{1}, z_{i}^{2}, z_{i}^{3}, x_{i}^{1}, x_{i}^{2}, x_{i}^{3}, c_{0}, c_{1}\right)$ and $R_{\mathrm{IL}_{2}}\left(x_{i}^{4}, x_{i}^{5}, x_{i}^{6}, x_{i}^{1}, x_{i}^{2}, x_{i}^{3}, c_{0}, c_{1}\right)$. Since every variable in $V$ can occur in at most two constraints we have that $|C| \leq$ $2|V|$. Hence, the resulting U-MAX-ONES $\left(\left\{R_{\mathrm{IL}_{2}}\right\}\right)$ instance $\left(V^{\prime}, C^{\prime}\right)$ contains at most $2+2|V|+3 \cdot 2|V|=2+8|V|$ variables. Now, in any satisfying assignment to $\left(V^{\prime}, C^{\prime}\right)$, it holds (1) that $v_{0}$ and $v_{1}$ must be assigned different values and (2) every $x \in V$ has a complementary variable $x^{\prime} \in V^{\prime}$ which must be assigned a different value. It therefore follows that the measure of a solution of $\left(V^{\prime}, C^{\prime}\right)$ is exactly the number of variables $z_{i}^{j} \in V^{\prime}$ that are mapped to 1 . Hence, for an optimal solution the objective value is larger than or equal to $2|C|$ if and only if $I$ is satisfiable.

Lemma 8.15. U-MAX-ONES $\left(\left\{R_{\mathrm{IL}_{2}}\right\}\right)$ LV-reduces to U-MAX-ONES $\left(\left\{R_{\mathrm{IL}_{0}}\right\}\right)$.

Proof. We reduce an instance $I=(V, C)$ of U-MAX-ONES $\left(\left\{R_{\mathrm{IL}_{2}}\right\}\right)$ to an instance of U-MAX-ONES $\left(\left\{R_{\mathrm{IL}_{0}}\right\}\right)$ on $2+2|V|$ variables. Let $v_{0}, v_{1}, y_{1}, \ldots, y_{|V|}$ be fresh variables and constrain them as $R_{\mathrm{IL}_{0}}\left(v_{0}, v_{0}, v_{0}, v_{0}\right) \wedge R_{\mathrm{IL}_{0}}\left(v_{1}, v_{0}, y_{1}, v_{0}\right) \wedge \ldots \wedge R_{\mathrm{IL}_{0}}\left(v_{1}, v_{0}, y_{|V|}, v_{0}\right)$. Note that this forces $v_{0}$ to 0 , and that if $v_{1}$ is mapped to 0 , then so are the variables $y_{1}, \ldots, y_{|V|}$. On the other hand, if $v_{1}$ is mapped to 1 , then $y_{1}, \ldots, y_{|V|}$ can be mapped to 1 . For every constraint $R_{\mathrm{IL}_{2}}\left(x_{i}^{1}, x_{i}^{2}, x_{i}^{3}, x_{i}^{4}, x_{i}^{5}, x_{i}^{6}, c_{0}, c_{1}\right)$ in $C$ we create the constraints $R_{\mathrm{IL}_{0}}\left(x_{i}^{1}, x_{i}^{2}, x_{i}^{3}, v_{0}\right) \wedge R_{\mathrm{IL}_{0}}\left(v_{1}, x_{i}^{1}, x_{i}^{4}, v_{0}\right) \wedge$ $R_{\mathrm{IL}_{0}}\left(v_{1}, x_{i}^{2}, x_{i}^{5}, v_{0}\right) \wedge R_{\mathrm{IL}_{0}}\left(v_{1}, x_{i}^{3}, x_{i}^{6}, v_{0}\right) \wedge R_{\mathrm{IL}_{0}}\left(v_{1}, c_{0}, c_{1}, v_{0}\right)$. It follows that the above reduction is an LV-reduction since the resulting U-MAX-ONES $\left(\left\{R_{\mathrm{IL}_{0}}\right\}\right)$ instance has $2+2|V|$ variables and has a solution with measure $|V|+1+k$ if and only if $I$ has a solution with measure $k$.

Lemma 8.16. U-MAX-ONES $\left(\left\{R_{I_{2}}\right\}\right)$ LV-reduces to U-MAX-ONES $\left(\left\{R_{I_{2}}\right\}\right)$.

Proof. We reduce an instance $I=(V, C)$ of U-MAX-ONES $\left(\left\{R_{\mathrm{II}_{2}}\right\}\right)$ to an instance of U-MAX-ONES $\left(\left\{R_{\mathrm{IN}_{2}}\right\}\right)$ over $2+3|V|$ variables. Create two fresh variables $v_{0}, v_{1}$ and constrain them as $R_{\mathrm{IN}_{2}}\left(v_{0}, v_{0}, v_{0}, v_{0}, v_{1}, v_{1}, v_{1}, v_{1}\right)$ in order to force $v_{0}$ and $v_{1}$ to be mapped to different values. We then create the $2|V|$ variables $y_{1}, \ldots, y_{2}|V|$ and constrain them as $\bigwedge_{i=1}^{2|V|} R_{\mathrm{IN}_{2}}\left(v_{0}, v_{0}, v_{0}, v_{0}, y_{i}, y_{i}, y_{i}, y_{i}\right)$. This forces all of the variables $y_{i}$ to be mapped to the same value as $v_{1}$. We can now express each constraint $R_{\mathrm{II}_{2}}\left(x_{i}^{1}, x_{i}^{2}, x_{i}^{3}, x_{i}^{4}, x_{i}^{5}, x_{i}^{6}, c_{0}, c_{1}\right)$ in $C$ with the implementation $R_{\mathrm{IN}_{2}}\left(v_{0}, x_{i}^{1}, x_{i}^{2}, x_{i}^{6}, v_{1}, x_{i}^{4}, x_{i}^{5}, x_{i}^{3}\right) \wedge R_{\mathrm{IN}_{2}}\left(v_{0}, c_{0}, c_{0}, v_{0}, v_{1}, c_{1}, c_{1}, v_{1}\right)$. Note that in any optimal solution of the new instance $v_{1}$ will be mapped to 1 which means that the implementation of $R_{\mathrm{II}_{2}}$ given above will be correct. The resulting instance has a solution with measure $1+2|V|+k$ if and only if $I$ has a solution with measure $k$.

Lemma 8.17. U-MAX-ONES $\left(\left\{R_{\mathrm{IS}_{1}^{2}}\right\}\right)$ LV-reduces to U-MAX-ONES $\left(\left\{R_{\mathrm{ID}_{2}}\right\}\right)$. 
Proof. We reduce an instance $I=(V, C)$ of U-MAX-ONES $\left(\left\{R_{\mathrm{IS}_{1}^{2}}\right\}\right)$ to an instance of U-MAX-ONES $\left(\left\{R_{\mathrm{ID}_{2}}\right\}\right)$ on $2+3|V|$ variables. Create two new variables $v_{0}$ and $v_{1}$ and constrain them as $R_{\mathrm{ID}_{2}}\left(v_{1}, v_{1}, v_{0}, v_{0}, v_{0}, v_{1}\right)$. Note that this forces $v_{0}$ to 0 and $v_{1}$ to 1 . For every variable $x \in$ $V$ we introduce two extra variables $x^{\prime}$ and $x^{\prime \prime}$ and constrain them as $R_{\mathrm{ID}_{2}}\left(x, x^{\prime}, x^{\prime}, x, v_{0}, v_{1}\right) \wedge R_{\mathrm{ID}_{2}}\left(x^{\prime}, x^{\prime \prime}, x^{\prime \prime}, x^{\prime}, v_{0}, v_{1}\right)$. Note that this implements the constraints $\operatorname{Neq}\left(x, x^{\prime}\right)$ and $\operatorname{Neq}\left(x^{\prime}, x^{\prime \prime}\right)$, and that no matter what $x$ is mapped to exactly one of $x^{\prime}$ and $x^{\prime \prime}$ is mapped to 1 . For every constraint $R_{\mathrm{IS}_{1}^{2}}\left(x_{i}, y_{i}, c_{0}\right)$ in $C$ we then introduce the constraint $R_{\mathrm{ID}_{2}}\left(x_{i}^{\prime}, y_{i}^{\prime}, x_{i}, y_{i}, c_{0}, v_{1}\right)$. The resulting instance has a solution with measure $1+|V|+k$ if and only if $I$ has a solution with measure $k$.

Lemma 8.18. U-MAX-ONES $\left(\left\{R_{\mathrm{LL}_{2}}\right\}\right) L V$-reduces to U-MAX-ONES $\left(\left\{R_{\mathrm{IL}_{3}}\right\}\right)$.

Proof. We reduce an instance $I=(V, C)$ of U-MAX-ONES $\left(\left\{R_{\mathrm{IL}_{2}}\right\}\right)$ to an instance of U-MAX-ONES $\left(\left\{R_{\mathrm{IL}_{3}}\right\}\right)$ on $2+3|V|$ variables. Create two new variables $v_{0}$ and $v_{1}$ and constrain them as $R_{\mathrm{IL}_{3}}\left(v_{0}, v_{0}, v_{0}, v_{0}, v_{1}, v_{1}, v_{1}, v_{1}\right)$. Note that this forces $v_{0}$ and $v_{1}$ to be mapped to different values. We then introduce fresh variables $y_{1}, \ldots, y_{2}|V|$ and constrain them as $\bigwedge_{i=1}^{2|V|} R_{\mathrm{IL}_{3}}\left(v_{0}, v_{0}, v_{0}, v_{0}, y_{i}, y_{i}, y_{i}, y_{i}\right)$. This will ensure that every variables $y_{i}$ is mapped to the same value as $v_{1}$ and therefore that in every optimal solution $v_{0}$ is mapped to 0 and $v_{1}$ is mapped to 1. For every constraint $R_{\mathrm{IL}_{2}}\left(x_{i}^{1}, \ldots, x_{i}^{6}, c_{0}, c_{1}\right)$ in $C$ we introduce the constraints $R_{\mathrm{IL}_{3}}\left(c_{0}, x_{i}^{1}, x_{i}^{2}, x_{i}^{3}, c_{1}, x_{i}^{4}, x_{i}^{5}, x_{i}^{6}\right) \wedge R_{\mathrm{IL}_{3}}\left(c_{0}, c_{0}, c_{0}, c_{0}, v_{1}, v_{1}, v_{1}, v_{1}\right) \wedge$ $R_{\mathrm{IL}_{3}}\left(v_{0}, v_{0}, v_{0}, v_{0}, c_{1}, c_{1}, c_{1}, c_{1}\right)$. The resulting instance has a solution with measure $1+2|V|+k$ if and only if $I$ has a solution with measure $k$. 


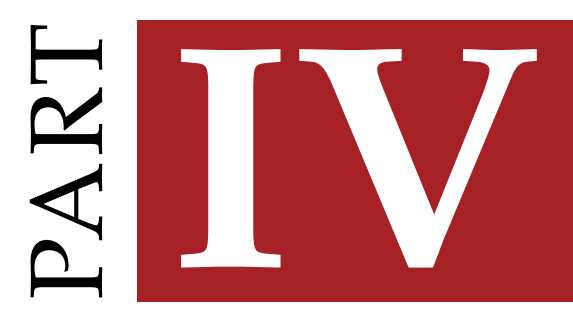

Conclusion 



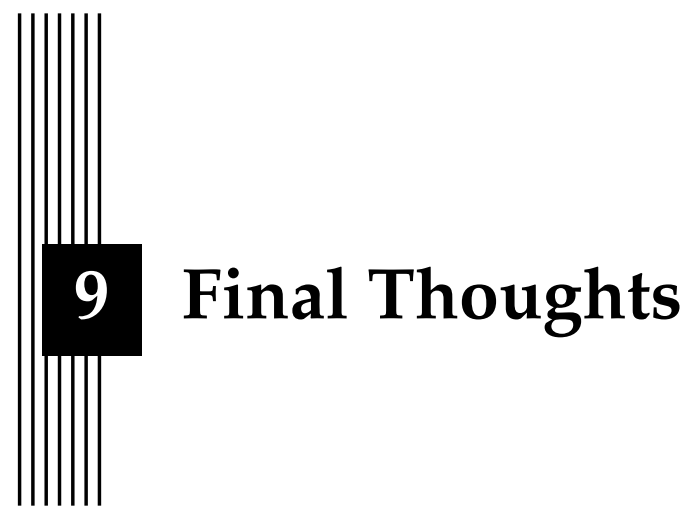

- Never I had seen the parlour so full.

- This actor he make very well her part.

- That piece is full of interest.

- It have wondered the spectadors.

- The curtains let down.

- Go out us.

English as she is spoke

Since the end of this thesis is rapidly approaching, it is time to close the curtains and give a brief summary of what we have learned so far. We have studied the complexity of $\operatorname{SAT}(\cdot)$ and related problems using a slightly nonorthodox Galois connection, which allowed us to get a more refined view of the worst-case complexity of SAT $(\cdot)$ than possible with the Galois connection between total clones and co-clones. In the process we have also studied properties of strong partial clones. On the one hand, we gave a classification of weak bases of all Boolean co-clones, that proved to have many applications throughout the thesis. On the other hand, we quickly learned a severe limitation of partial clone theory, namely that essentially all strong partial clones of interest in this thesis are of infinite order. However, despite this slightly depressing fact, we were still able to prove that it is possible to describe these strong partial clones using bounded bases. 
We now discuss some open research questions that have been left dangling.

\subsection{When are Strong Partial Clones of Infinite Order?}

From the results in Chapter 4 we know that $\mathrm{pPol}(S)$ is of infinite order whenever $\operatorname{Pol}(S)$ is essentially unary and $S$ is finite. Can stronger results be obtained? A reasonable conjecture is that $\operatorname{pPol}(S)$ is of infinite order whenever $S$ is a finite constraint language over a finite domain $D$ and the algebra $(D, \operatorname{Pol}(S))$ has many subpowers, i.e., $\operatorname{Pol}(S)$ does not contain an $n$-edge operation for any $n \geq 2$. As a starting point, one could attempt to prove that this conjecture is true for all superpolynomially closed Boolean co-clones. However, this appears to be far from straightforward, and even in the appearingly simple case when $\operatorname{Pol}(S)=\left[\left\{x_{1} \wedge x_{2}\right\}\right]$, the proof strategy in Lemma 4.29 in Chapter 4 , based on constructing a universal hash family, breaks down. Hence, it is likely that entirely new constructions are needed for such cases.

\subsection{Covering Results for Strong Partial Clones}

In Chapter 7 we provided an incomplete classification of constraint languages $S$ satisfying $\langle S\rangle=\mathrm{BR}$ and $\langle S\rangle_{\nexists} \subseteq\left\langle\Gamma_{1 / \kappa}\right\rangle_{\nexists}$. It would be interesting to investigate to which extent this classification is correct. In particular, is it the case that the four weak partial co-clones $\left\langle\left\{R_{1 / 3}\right\}\right\rangle_{\nexists \prime},\left\langle\left\{R_{1 / 3}^{01}\right\}\right\rangle_{\nexists \prime},\left\langle\left\{R_{1 / 3}^{\neq 01}\right\}\right\rangle_{\nexists \prime}$ $\left\langle\left\{R_{1 / 3}^{\neq \neq 01}\right\}\right\rangle_{\nexists \prime},\left\langle\left\{R_{1 / 3}^{\neq \neq \neq 01}\right\}\right\rangle_{\exists}$ in Figure 7.6 cover each other in the sense that it is not possible to find any weak partial co-clone between them? Proving this from the relational side via q.f.p.p. definitions appears extremely complicated. On the other hand, since all the corresponding strong partial clones are of infinite order, it does not appear easy to prove via partial polymorphisms either. One possible way to mitigate this is to try to describe the partial polymorphisms of these relations using the concept of bounded bases, developed in Chapter 5 .

\subsection{Easy Problems and Subexponential Time}

To our knowledge, the results in Chapter 7 and Chapter 8 , where we show the existence of 'easiest problems' with respect to CV-reductions, are the first of their kind. These problems provide a natural method to prove lower bounds via the ETH, since, if we can prove that the easiest problem is solvable in subexponential time if and only if 3-SAT is solvable in subexponential time, then the ETH fails if and only if there exists a single NP-complete problem in this class solvable in subexponential time. However, in the case of $\operatorname{SAT}(\cdot)$, our results are much more precise, since we not only know that $\operatorname{SAT}\left(\left\{R_{1 / 3}^{\neq \neq \neq}\right\}\right)$is the easiest NP-complete $\operatorname{SAT}(\cdot)$ problem, but that it is not that much easier than 1-IN-3-SAT, since it is solvable in $O\left(2^{c|V|}\right)$ time if and only if 1 -IN-3-SAT is solvable in $O\left(2^{2 c|V|}\right)$ time. Hence, no matter which 
bizarre Boolean constraint language one can conjecture, it is still not possible to solve the resulting SAT $(\cdot)$ problem much faster than 1-IN-3-SAT.

Lower bounds of this form are interesting since it highlights the probability of subexponential algorithms in light of the ETH. Hence, even if one does not believe that the ETH is plausible, it is informative to know that (1) a subexponential time algorithm for 3-SAT immediately implies that many other problems are solvable in subexponential time, and (2) if 3-SAT is not solvable in subexponential time then this implies that it is impossible to solve a large range of NP-complete problems in subexponential time.

In light of this a reasonable continuation of the research of this thesis is to investigate larger classes of problems, and see when it is possible to obtain an easiest problem with respect to CV-reductions. A possible starting point is to consider $\operatorname{CSP}(S)$ where $S$ is a finite constraint language over a ternary domain, since a complete dichotomy conjecture has been established [Bul06]. However, since the lattice of co-clones over a ternary domain is of uncountably infinite cardinality, the approach in Chapter 7 based on systematically comparing all NP-complete cases, might not be directly applicable. 



\section{Bibliography}

[AV94] V. B. Alekseev and A. A. Voronenko. "On some closed classes in partial two-valued logic". English. In: Discrete Mathematics and Applications 4.5 (1994), pp. 401-419 (cit. on p. 37).

[AYZ95] N. Alon, R. Yuster, and U. Zwick. "Color-coding". In: Journal of the ACM 42.4 (1995), pp. 844-856 (cit. on pp.72, 73).

[B0̈8] F. Börner. "Basics of Galois Connections". English. In: Complexity of Constraints. Ed. by N. Creignou, P. G. Kolaitis, and H. Vollmer. Vol. 5250. Lecture Notes in Computer Science. Springer Berlin Heidelberg, 2008, pp. 38-67 (cit. on p. 30).

[BCRV03] E. Böhler, N. Creignou, S. Reith, and H. Vollmer. "Playing with Boolean Blocks, Part I: Post's Lattice with Applications to Complexity Theory". In: ACM SIGACT-Newsletter 34.4 (2003), pp. 38-52 (cit. on p. 26.

[BH98] F. Börner and L. Haddad. "Maximal partial clones with no finite basis". English. In: Algebra Universalis 40.4 (1998), pp. 453-476 (cit. on pp. 58, 69).

[BHP91] F. Börner, L. Haddad, and R. Pöschel. "A note on minimal partial clones". In: Proceedings of the 21th International Symposium on Multiple-Valued Logic (ISMVL-1991). 1991, pp. 262267 (cit. on p. 38).

[BKKR69a] V. G. Bodnarchuk, L. A. Kaluzhnin, V. N. Kotov, and B. A. Romov. "Galois theory for Post algebras. I". In: Cybernetics 5.3 (1969), pp. 243-252 (cit. on pp. 16. 17, 34, 35. 
[BKKR69b] V. G. Bodnarchuk, L. A. Kaluzhnin, V. N. Kotov, and B. A. Romov. "Galois theory for Post algebras. II". In: Cybernetics 5.5 (1969), pp. 531-539 (cit. on pp. 16, 17, $34,35$.

[BMMVW06] J. Berman, P. Marković, R. Mckenzie, M. Valeriote, and R. Willard. "Varieties with few subalgebras of powers". In: Transactions of the American Mathematical Society 362.3 (2006), pp. $1445-1473$ (cit. on pp. 20, 58, 66, 67).

[Bod12] M. Bodirsky. Complexity Classification in Infinite-Domain Constraint Satisfaction. Mémoire d'habilitation à diriger des recherches, Université Diderot - Paris 7. Available at arXiv:1201.0856. 2012 (cit. on p. 37).

[BS81] S. Burris and H. Sankappanavar. A course in universal algebra. Graduate texts in mathematics. Springer-Verlag, 1981 (cit. on p.15.

[BSRV05] E. Böhler, H. Schnoor, S. Reith, and H. Vollmer. "Bases for Boolean co-clones". In: Information Processing Letters $\mathbf{9 6 . 2}$ (2005), pp. 59-66 (cit. on pp. 31. 44, 53).

[Bul06] A. Bulatov. "A dichotomy theorem for constraint satisfaction problems on a 3-element set". In: Journal of the ACM 53.1 (2006), pp. 66-120 (cit. on pp. 14, 153).

[CCJK06] D. A. Cohen, M. C. Cooper, P. G. Jeavons, and A. A. Krokhin. "The complexity of soft constraint satisfaction". In: Artificial Intelligence 170.11 (2006), pp. 983-1016 (cit. on pp. 134. 140. 141).

[CH10] M. Couceiro and L. Haddad. "Finitely Generated Maximal Partial Clones and Their Intersections". In: Proceedings of the 40th IEEE International Symposium on Multiple-Valued Logic, (ISMVL-2010). IEEE Computer Society, 2010, pp. 122-127 (cit. on pp. 69, 72).

[CKZ08] N. Creignou, P. Kolaitis, and B. Zanuttini. "Structure identification of Boolean relations and plain bases for co-clones". In: Journal of Computer and System Sciences 74.7 (2008), pp. 11031115 (cit. on pp. 44, 47, 51, 53, 58, 59, 65.

[Coh81] P. Cohn. Universal Algebra. Mathematics and its applications. Springer Netherlands, 1981 (cit. on p. 16).

[CV08] N. Creignou and H. Vollmer. "Boolean Constraint Satisfaction Problems: When Does Post's Lattice Help?" In: Complexity of Constraints. Ed. by N. Creignou, P. G. Kolaitis, and H. Vollmer. Vol. 5250. Lecture Notes in Computer Science. Springer Berlin Heidelberg, 2008, pp. 3-37 (cit. on p. 36.

[DEW13] K. Denecke, M. Erné, and S. Wismath. Galois Connections and Applications. Mathematics and Its Applications. Springer Netherlands, 2013 (cit. on pp. 16, 17). 
[DF03]

[DM10]

[Ern85]

[FH98]

[FV98]

[Gei68]

[Her14]

[HF99]

[IP01]

[IPZ01]
V. Dalmau and D. Ford. "Generalized Satisfiability with Limited Occurrences per Variable: A Study through DeltaMatroid Parity". In: Proceedings of the 28th International Symposium on Mathematical Foundations of Computer Science (MFCS-2003). 2003, pp. 358-367 (cit. on pp.102,120).

H. Dell and D. van Melkebeek. "Satisfiability allows no nontrivial sparsification unless the polynomial-time hierarchy collapses". In: Proceedings of the 42nd ACM symposium on Theory of computing (STOC-2010). 2010, pp. 251-260 (cit. on pp. 121. 127).

M. Erné. Ordnungs- und Verbandstheorie. Fernuniversität Hagen, 1985 (cit. on p.17).

J. Fugère and L. Haddad. "On Partial Clones Containing All Idempotent Partial Operations". In: Proceedings of the 28th IEEE International Symposium on Multiple-Valued Logic (ISMVL-1998). 1998, pp. 369-373 (cit. on p.72).

T. Feder and M. Vardi. "The Computational Structure of Monotone Monadic SNP and Constraint Satisfaction: A Study through Datalog and Group Theory". In: SIAM Journal on Computing 28.1 (1998), pp. 57-104 (cit. on p. 143).

D. Geiger. "Closed Systems of Functions and Predicates". In: Pacific Journal of Mathematics 27.1 (1968), pp. 95-100 (cit. on pp. $16,18,34,35,39$.

T. Hertli. "3-SAT Faster and Simpler - Unique-SAT Bounds for PPSZ Hold in General". In: SIAM Journal on Computing 43.2 (2014), pp. 718-729 (cit. on p. 40).

L. Haddad and J. Fugère. "Maximal Chains of Partial Clones Containing All Idempotent Partial Functions". In: Proceedings of the 29th IEEE International Symposium on Multiple-Valued Logic (ISMVL-99). IEEE Computer Society, 1999, pp. 80-84 (cit. on p. 69).

[IMMVW10] P. Idziak, P. Marković, R. McKenzie, M. Valeriote, and R. Willard. "Tractability and Learnability Arising from Algebras with Few Subpowers". In: SIAM Journal on Computing 39.7 (2010), pp. 3023-3037 (cit. on p. 66.

R. Impagliazzo and R. Paturi. "On the Complexity of k-SAT". In: Journal of Computer and System Sciences 62.2 (2001), pp. 367 -375 (cit. on pp. 96, 97, 101, 102, 110, 144).

R. Impagliazzo, R. Paturi, and F. Zane. "Which Problems Have Strongly Exponential Complexity?" In: Journal of Computer and System Sciences 63.4 (2001), pp. 512-530 (cit. on pp. 19, $96,101,102,121,122,127$. 
[Ist97] G. I. Istrate. Looking for a Version of Schaefer's Dichotomy Theorem When Each Variable Occurs at Most Twice. Tech. rep. 1997 (cit. on p.118).

[JCC98] P. Jeavons, D. Cohen, and M. C. Cooper. "Constraints, consistency and closure". In: Artificial Intelligence 101.1-2 (1998), pp. $251-265$ (cit. on p. 63).

[JCG97] P. Jeavons, D. Cohen, and M. Gyssens. “Closure Properties of Constraints". In: Journal of the ACM 44.4 (1997), pp. 527-548 (cit. on pp.17,75).

[Jea98] P. Jeavons. "On The Algebraic Structure Of Combinatorial Problems". In: Theoretical Computer Science 200.1-2 (1998), pp. 185-204 (cit. on pp. 35, 130.

[JKK09] P. Jonsson, A. Krokhin, and F. Kuivinen. "Hard constraint satisfaction problems have hard gaps at location 1". In: Theoretical Computer Science 410.38-40 (2009), pp. 3856-3874 (cit. on p. 103.

[JKT11] P. Jonsson, F. Kuivinen, and J. Thapper. "Min CSP on Four Elements: Moving beyond Submodularity". In: Proceedings of the Principles and Practice of Constraint Programming (CP-2011). Perugia, Italy: Springer Berlin Heidelberg, 2011, pp. 438-453 (cit. on pp.134, 138).

[KPS] S. Kerkhoff, R. Pöschel, and F. Schneider. "A Short Introduction to Clones". In: Proceedings of the Workshop on Algebra, Coalgebra and Topology (WACT-2013), pp. $107-120$ (cit. on p. 16.

[KSTW00] S. Khanna, M. Sudan, L. Trevisan, and D. Williamson. "The Approximability of Constraint Satisfaction Problems". In: SIAM Journal on Computing 30.6 (2000), pp. 1863-1920 (cit. on pp. 134, 135, 137).

[Lad75] R. E. Ladner. "On the Structure of Polynomial Time Reducibility". In: Journal of the ACM 22.1 (1975), pp. 155-171 (cit. on pp.14, 101.

[Lag14] V. Lagerkvist. Weak Bases. https://github.com/Joelbyte/ weak-bases 2014 (cit. on p.47).

[Lau06] D. Lau. Function Algebras on Finite Sets. Springer, Berlin, 2006 (cit. on p.38).

[LMS11] D. Lokshtanov, D. Marx, and S. Saurabh. "Lower bounds based on the Exponential Time Hypothesis". In: Bulletin of the EATCS 105 (2011), pp. 41-72 (cit. on p.101).

[MR01] C. Moore and J. M. Robson. "Hard Tiling Problems with Simple Tiles". In: Discrete E Computational Geometry 26.4 (2001), pp. 573-590 (cit. on p.118). 
[NZ09] G. Nordh and B. Zanuttini. "Frozen Boolean Partial Coclones". In: Proceedings of the 39th International Symposium on Multiple-Valued Logic (ISMVL-2009). 2009, pp. 120 -125 (cit. on pp. 58, 86.

[Pap94] C. H. Papadimitriou. Computational complexity. AddisonWesley, 1994 (cit. on pp. 5, 10).

[Pos41] E. Post. "The two-valued iterative systems of mathematical logic". In: Annals of Mathematical Studies 5 (1941), pp. 1-122 (cit. on p.26).

[Qua71] R. W. Quackenbush. "On the composition of idempotent functions". English. In: Algebra Universalis 1.1 (1971), pp. 712 (cit. on p.72).

[RKNB13] J. van Rooij, M. van Kooten Niekerk, and H. Bodlaender. "Partition Into Triangles on Bounded Degree Graphs". In: Theory of Computing Systems 52.4 (2013), pp. 687-718 (cit. on p. (100).

[Rom81] B. A. Romov. "The algebras of partial functions and their invariants". In: Cybernetics and Systems Analysis 17.2 (1981), pp. 157-167 (cit. on pp. 18, 39).

[Ros70] I. Rosenberg. Über die funktionale Vollständigkeit in den mehrwertigen Logiken: Struktur Der Funktionen Von Mehreren Veränderlichen Auf Endlichen Mengen. Praha: Academia, 1970 (cit. on p. 16.

[Ros86] I. Rosenberg. Minimal clones I: the five types. Colloquia mathematica Societatis János Bolyai. 1986, pp. 405-427 (cit. on p.16.

[RW99] S. Reith and K. W. Wagner. "The Complexity of Problems Defined by Boolean Circuits". In: Proceedings International Conference Mathematical Foundation of Informatics (MFI-1999). 1999, pp. 25-28 (cit. on p. 26).

[Sch08] I. Schnoor. "The weak base method for constraint satisfaction". PhD thesis. Hannover, Germany: Gottfried Wilhelm Leibniz Universität, 2008, pp. 1-107 (cit. on pp.4446).

[Sch15] K. Schölzel. "Dichotomy on intervals of strong partial Boolean clones". In: Algebra Universalis 73.3-4 (2015), pp. 347368 (cit. on pp. 44, 70, 72, 77, 110).

[Sch78] T. J. Schaefer. "The complexity of satisfiability problems". In: Proceedings of the tenth annual ACM symposium on Theory of computing (STOC-1978). San Diego, California, United States: ACM, 1978, pp. 216-226 (cit. on pp. 14, 35).

[Sch83] U. Schöning. "A low and a high hierarchy within NP". In: Journal of Computer and System Sciences 27.1 (1983), pp. $14-28$ (cit. on p. 101). 
[SS08]

[SS12]

[Tha10] J. Thapper. "Aspects of a Constraint Optimisation Problem". $\mathrm{PhD}$ thesis. Linköping University, The Institute of Technology, 2010, p. 218 (cit. on pp. 134. 138).

[TŽ13] J. Thapper and S. Živný. "The Complexity of Finite-valued CSPs". In: Proceedings of the 45th Annual ACM Symposium on Symposium on Theory of Computing (STOC-2013). ACM, 2013, pp. 695-704 (cit. on p. 134).

[Wah07] M. Wahlström. "Algorithms, measures and upper bounds for satisfiability and related problems". PhD thesis. Linköping University, The Institute of Technology, 2007, p. 234 (cit. on p. 40.

[Woe] G. J. Woeginger. The P-versus-NP page. http://www win. tue. nl/ gwoegi/P-versus-NP.htm. Accessed: 2015-10-29 (cit. on p. 11].

[Woe03] G. J. Woeginger. “Exact Algorithms for NP-Hard Problems: A Survey". In: Combinatorial Optimization - Eureka, You Shrink! Vol. 2570. Lecture Notes in Computer Science. 2003, pp. 185208 (cit. on p.101).

[YM59] Y. Yanov and A. A. Muchnik. "Existence of k-valued closed classes without a finite basis". In: Proceedings of the USSR Academy of Sciences 127.1 (1959), pp. 44-46 (cit. on p. 26). 


\section{Papers}

The articles associated with this thesis have been removed for copyright reasons. For more details about these see:

http://urn.kb.se/resolve?urn=urn:nbn:se:liu:diva-122827 


\section{Dissertations}

\section{Linköping Studies in Science and Technology Linköping Studies in Arts and Science}

Linköping Studies in Statistics

Linköpings Studies in Information Science

\section{Linköping Studies in Science and Technology}

No 14 Anders Haraldsson: A Program Manipulation System Based on Partial Evaluation, 1977, ISBN 91-7372-144-1.

No 17 Bengt Magnhagen: Probability Based Verification of Time Margins in Digital Designs, 1977, ISBN 91-7372157-3.

No 18 Mats Cedwall: Semantisk analys av processbeskrivningar i naturligt språk, 1977, ISBN 91- 7372-168-9.

No 22 Jaak Urmi: A Machine Independent LISP Compiler and its Implications for Ideal Hardware, 1978, ISBN 91-7372188-3.

No 33 Tore Risch: Compilation of Multiple File Queries in a Meta-Database System 1978, ISBN 91- 7372-232-4.

No 51 Erland Jungert: Synthesizing Database Structures from a User Oriented Data Model, 1980, ISBN 91-7372-387-8.

No 54 Sture Hägglund: Contributions to the Development of Methods and Tools for Interactive Design of Applications Software, 1980, ISBN 91-7372-404-1.

No 55 Pär Emanuelson: Performance Enhancement in a WellStructured Pattern Matcher through Partial Evaluation, 1980, ISBN 91-7372-403-3.

No 58 Bengt Johnsson, Bertil Andersson: The HumanComputer Interface in Commercial Systems, 1981, ISBN 91-7372-414-9.

No 69 H. Jan Komorowski: A Specification of an Abstract Prolog Machine and its Application to Partial Evaluation, 1981, ISBN 91-7372-479-3.

No 71 René Reboh: Knowledge Engineering Techniques and Tools for Expert Systems, 1981, ISBN 91-7372-489-0.

No 77 Östen Oskarsson: Mechanisms of Modifiability in large Software Systems, 1982, ISBN 91- 7372-527-7.

No 94 Hans Lunell: Code Generator Writing Systems, 1983, ISBN 91-7372-652-4.

No 97 Andrzej Lingas: Advances in Minimum Weight Triangulation, 1983, ISBN 91-7372-660-5.

No 109 Peter Fritzson: Towards a Distributed Programming Environment based on Incremental Compilation, 1984, ISBN 91-7372-801-2.

No 111 Erik Tengvald: The Design of Expert Planning Systems. An Experimental Operations Planning System for Turning, 1984, ISBN 91-7372- 805-5.

No 155 Christos Levcopoulos: Heuristics for Minimum Decompositions of Polygons, 1987, ISBN 91-7870-133-3.

No 165 James W. Goodwin: A Theory and System for NonMonotonic Reasoning, 1987, ISBN 91-7870-183-X.

No 170 Zebo Peng: A Formal Methodology for Automated Synthesis of VLSI Systems, 1987, ISBN 91-7870-225-9.

No 174 Johan Fagerström: A Paradigm and System for Design of Distributed Systems, 1988, ISBN 91-7870-301-8.

No 192 Dimiter Driankov: Towards a Many Valued Logic of Quantified Belief, 1988, ISBN 91-7870-374-3.

No 213 Lin Padgham: Non-Monotonic Inheritance for an Object Oriented Knowledge Base, 1989, ISBN 91-7870-485-5.

No 214 Tony Larsson: A Formal Hardware Description and Verification Method, 1989, ISBN 91-7870-517-7.

No 221 Michael Reinfrank: Fundamentals and Logical Foundations of Truth Maintenance, 1989, ISBN 91-7870546-0.
No 239 Jonas Löwgren: Knowledge-Based Design Support and Discourse Management in User Interface Management Systems, 1991, ISBN 91-7870-720-X.

No 244 Henrik Eriksson: Meta-Tool Support for Knowledge Acquisition, 1991, ISBN 91-7870-746-3.

No 252 Peter Eklund: An Epistemic Approach to Interactive Design in Multiple Inheritance Hierarchies, 1991, ISBN 91-7870-784-6.

No 258 Patrick Doherty: NML3 - A Non-Monotonic Formalism with Explicit Defaults, 1991, ISBN 91-7870-816-8.

No 260 Nahid Shahmehri: Generalized Algorithmic Debugging, 1991, ISBN 91-7870-828-1.

No 264 Nils Dahlbäck: Representation of Discourse-Cognitive and Computational Aspects, 1992, ISBN 91-7870-850-8.

No 265 Ulf Nilsson: Abstract Interpretations and Abstract Machines: Contributions to a Methodology for the Implementation of Logic Programs, 1992, ISBN 91-7870858-3.

No 270 Ralph Rönnquist: Theory and Practice of Tense-bound Object References, 1992, ISBN 91-7870-873-7.

No 273 Björn Fjellborg: Pipeline Extraction for VLSI Data Path Synthesis, 1992, ISBN 91-7870-880-X.

No 276 Staffan Bonnier: A Formal Basis for Horn Clause Logic with External Polymorphic Functions, 1992, ISBN 917870-896-6.

No 277 Kristian Sandahl: Developing Knowledge Management Systems with an Active Expert Methodology, 1992, ISBN 91-7870-897-4.

No 281 Christer Bäckström: Computational Complexity of Reasoning about Plans, 1992, ISBN 91-7870-979-2.

No 292 Mats Wirén: Studies in Incremental Natural Language Analysis, 1992, ISBN 91-7871-027-8.

No 297 Mariam Kamkar: Interprocedural Dynamic Slicing with Applications to Debugging and Testing, 1993, ISBN 917871-065-0.

No 302 Tingting Zhang: A Study in Diagnosis Using Classification and Defaults, 1993, ISBN 91-7871-078-2

No 312 Arne Jönsson: Dialogue Management for Natural Language Interfaces - An Empirical Approach, 1993, ISBN 91-7871-110-X.

No 338 Simin Nadjm-Tehrani: Reactive Systems in Physical Environments: Compositional Modelling and Framework for Verification, 1994, ISBN 91-7871-237-8.

No 371 Bengt Savén: Business Models for Decision Support and Learning. A Study of Discrete-Event Manufacturing Simulation at Asea/ABB 1968-1993, 1995, ISBN 917871-494-X.

No 375 Ulf Söderman: Conceptual Modelling of Mode Switching Physical Systems, 1995, ISBN 91-7871-516-4.

No 383 Andreas Kågedal: Exploiting Groundness in Logic Programs, 1995, ISBN 91-7871-538-5.

No 396 George Fodor: Ontological Control, Description, Identification and Recovery from Problematic Control Situations, 1995, ISBN 91-7871-603-9.

No 413 Mikael Pettersson: Compiling Natural Semantics, 1995, ISBN 91-7871-641-1.

No 414 Xinli Gu: RT Level Testability Improvement by Testability Analysis and Transformations, 1996, ISBN 917871-654-3. 
No 416 Hua Shu: Distributed Default Reasoning, 1996, ISBN 917871-665-9.

No 429 Jaime Villegas: Simulation Supported Industrial Training from an Organisational Learning Perspective Development and Evaluation of the SSIT Method, 1996, ISBN 91-7871-700-0.

No 431 Peter Jonsson: Studies in Action Planning: Algorithms and Complexity, 1996, ISBN 91-7871-704-3.

No 437 Johan Boye: Directional Types in Logic Programming, 1996, ISBN 91-7871-725-6.

No 439 Cecilia Sjöberg: Activities, Voices and Arenas: Participatory Design in Practice, 1996, ISBN 91-7871728-0.

No 448 Patrick Lambrix: Part-Whole Reasoning in Description Logics, 1996, ISBN 91-7871-820-1.

No 452 Kjell Orsborn: On Extensible and Object-Relational Database Technology for Finite Element Analysis Applications, 1996, ISBN 91-7871-827-9.

No 459 Olof Johansson: Development Environments for Complex Product Models, 1996, ISBN 91-7871-855-4.

No 461 Lena Strömbäck: User-Defined Constructions in Unification-Based Formalisms, 1997, ISBN 91-7871-8570 .

No 462 Lars Degerstedt: Tabulation-based Logic Programming: A Multi-Level View of Query Answering, 1996, ISBN 917871-858-9.

No 475 Fredrik Nilsson: Strategi och ekonomisk styrning - En studie av hur ekonomiska styrsystem utformas och används efter företagsförvärv, 1997, ISBN 91-7871-914-3.

No 480 Mikael Lindvall: An Empirical Study of RequirementsDriven Impact Analysis in Object-Oriented Software Evolution, 1997, ISBN 91-7871-927-5.

No 485 Göran Forslund: Opinion-Based Systems: The Cooperative Perspective on Knowledge-Based Decision Support, 1997, ISBN 91-7871-938-0.

No 494 Martin Sköld: Active Database Management Systems for Monitoring and Control, 1997, ISBN 91-7219-002-7.

No 495 Hans Olsén: Automatic Verification of Petri Nets in a CLP framework, 1997, ISBN 91-7219-011-6.

No 498 Thomas Drakengren: Algorithms and Complexity for Temporal and Spatial Formalisms, 1997, ISBN 91-7219019-1.

No 502 Jakob Axelsson: Analysis and Synthesis of Heterogeneous Real-Time Systems, 1997, ISBN 91-7219-035-3.

No 503 Johan Ringström: Compiler Generation for Data-Parallel Programming Languages from Two-Level Semantics Specifications, 1997, ISBN 91-7219-045-0.

No 512 Anna Moberg: Närhet och distans - Studier av kommunikationsmönster i satellitkontor och flexibla kontor, 1997, ISBN 91-7219-119-8.

No 520 Mikael Ronström: Design and Modelling of a Parallel Data Server for Telecom Applications, 1998, ISBN 917219-169-4.

No 522 Niclas Ohlsson: Towards Effective Fault Prevention - An Empirical Study in Software Engineering, 1998, ISBN 917219-176-7.

No 526 Joachim Karlsson: A Systematic Approach for Prioritizing Software Requirements, 1998, ISBN 91-7219184-8.

No 530 Henrik Nilsson: Declarative Debugging for Lazy Functional Languages, 1998, ISBN 91-7219-197-x.

No 555 Jonas Hallberg: Timing Issues in High-Level Synthesis, 1998, ISBN 91-7219-369-7.

No 561 Ling Lin: Management of 1-D Sequence Data - From Discrete to Continuous, 1999, ISBN 91-7219-402-2.

No 563 Eva L Ragnemalm: Student Modelling based on Collaborative Dialogue with a Learning Companion, 1999, ISBN 91-7219-412-X.
No 567 Jörgen Lindström: Does Distance matter? On geographical dispersion in organisations, 1999, ISBN 917219-439-1.

No 582 Vanja Josifovski: Design, Implementation and Evaluation of a Distributed Mediator System for Data Integration, 1999, ISBN 91-7219-482-0.

No 589 Rita Kovordányi: Modeling and Simulating Inhibitory Mechanisms in Mental Image Reinterpretation - Towards Cooperative Human-Computer Creativity, 1999, ISBN 917219-506-1.

No 592 Mikael Ericsson: Supporting the Use of Design Knowledge - An Assessment of Commenting Agents, 1999, ISBN 91-7219-532-0.

No 593 Lars Karlsson: Actions, Interactions and Narratives, 1999, ISBN 91-7219-534-7.

No 594 C. G. Mikael Johansson: Social and Organizational Aspects of Requirements Engineering Methods - A practice-oriented approach, 1999, ISBN 91-7219-541-X.

No 595 Jörgen Hansson: Value-Driven Multi-Class Overload Management in Real-Time Database Systems, 1999, ISBN 91-7219-542-8.

No 596 Niklas Hallberg: Incorporating User Values in the Design of Information Systems and Services in the Public Sector: A Methods Approach, 1999, ISBN 91-7219-543-6.

No 597 Vivian Vimarlund: An Economic Perspective on the Analysis of Impacts of Information Technology: From Case Studies in Health-Care towards General Models and Theories, 1999, ISBN 91-7219-544-4.

No 598 Johan Jenvald: Methods and Tools in ComputerSupported Taskforce Training, 1999, ISBN 91-7219-5479.

No 607 Magnus Merkel: Understanding and enhancing translation by parallel text processing, 1999, ISBN 917219-614-9.

No 611 Silvia Coradeschi: Anchoring symbols to sensory data, 1999, ISBN 91-7219-623-8.

No 613 Man Lin: Analysis and Synthesis of Reactive Systems: A Generic Layered Architecture Perspective, 1999, ISBN 91-7219-630-0.

No 618 Jimmy Tjäder: Systemimplementering i praktiken - En studie av logiker i fyra projekt, 1999, ISBN 91-7219-6572.

No 627 Vadim Engelson: Tools for Design, Interactive Simulation, and Visualization of Object-Oriented Models in Scientific Computing, 2000, ISBN 91-7219-709-9.

No 637 Esa Falkenroth: Database Technology for Control and Simulation, 2000, ISBN 91-7219-766-8.

No 639 Per-Arne Persson: Bringing Power and Knowledge Together: Information Systems Design for Autonomy and Control in Command Work, 2000, ISBN 91-7219-796-X.

No 660 Erik Larsson: An Integrated System-Level Design for Testability Methodology, 2000, ISBN 91-7219-890-7.

No 688 Marcus Bjäreland: Model-based Execution Monitoring, 2001, ISBN 91-7373-016-5.

No 689 Joakim Gustafsson: Extending Temporal Action Logic, 2001, ISBN 91-7373-017-3.

No 720 Carl-Johan Petri: Organizational Information Provision Managing Mandatory and Discretionary Use of Information Technology, 2001, ISBN-91-7373-126-9.

No 724 Paul Scerri: Designing Agents for Systems with Adjustable Autonomy, 2001, ISBN 9173732079.

No 725 Tim Heyer: Semantic Inspection of Software Artifacts: From Theory to Practice, 2001, ISBN 9173732087.

No 726 Pär Carlshamre: A Usability Perspective on Requirements Engineering - From Methodology to Product Development, 2001, ISBN 9173732125.

No 732 Juha Takkinen: From Information Management to Task Management in Electronic Mail, 2002, ISBN 917373258 3 . 
No 745 Johan Åberg: Live Help Systems: An Approach to Intelligent Help for Web Information Systems, 2002, ISBN 91-7373-311-3.

No 746 Rego Granlund: Monitoring Distributed Teamwork Training, 2002, ISBN 91-7373-312-1.

No 757 Henrik André-Jönsson: Indexing Strategies for Time Series Data, 2002, ISBN 917373-346-6.

No 747 Anneli Hagdahl: Development of IT-supported Interorganisational Collaboration - A Case Study in the Swedish Public Sector, 2002, ISBN 91-7373-314-8.

No 749 Sofie Pilemalm: Information Technology for Non-Profit Organisations - Extended Participatory Design of an Information System for Trade Union Shop Stewards, 2002, ISBN 91-7373-318-0.

No 765 Stefan Holmlid: Adapting users: Towards a theory of use quality, 2002, ISBN 91-7373-397-0.

No 771 Magnus Morin: Multimedia Representations of Distributed Tactical Operations, 2002, ISBN 91-7373-421-7.

No 772 Pawel Pietrzak: A Type-Based Framework for Locating Errors in Constraint Logic Programs, 2002, ISBN 917373-422-5.

No 758 Erik Berglund: Library Communication Among Programmers Worldwide, 2002, ISBN 91-7373-349-0.

No 774 Choong-ho Yi: Modelling Object-Oriented Dynamic Systems Using a Logic-Based Framework, 2002, ISBN 91-7373-424-1.

No 779 Mathias Broxvall: A Study in the Computational Complexity of Temporal Reasoning, 2002, ISBN 91-7373440-3.

No 793 Asmus Pandikow: A Generic Principle for Enabling Interoperability of Structured and Object-Oriented Analysis and Design Tools, 2002, ISBN 91-7373-479-9.

No 785 Lars Hult: Publika Informationstjänster. En studie av den Internetbaserade encyklopedins bruksegenskaper, 2003, ISBN 91-7373-461-6.

No 800 Lars Taxén: A Framework for the Coordination of Complex Systems' Development, 2003, ISBN 91-7373604-X

No 808 Klas Gäre: Tre perspektiv på förväntningar och förändringar i samband med införande av informationssystem, 2003, ISBN 91-7373-618-X.

No 821 Mikael Kindborg: Concurrent Comics - programming of social agents by children, 2003, ISBN 91-7373-651-1.

No 823 Christina Ölvingson: On Development of Information Systems with GIS Functionality in Public Health Informatics: A Requirements Engineering Approach, 2003, ISBN 91-7373-656-2.

No 828 Tobias Ritzau: Memory Efficient Hard Real-Time Garbage Collection, 2003, ISBN 91-7373-666-X.

No 833 Paul Pop: Analysis and Synthesis of CommunicationIntensive Heterogeneous Real-Time Systems, 2003, ISBN 91-7373-683-X.

No 852 Johan Moe: Observing the Dynamic Behaviour of Large Distributed Systems to Improve Development and Testing - An Empirical Study in Software Engineering, 2003, ISBN 91-7373-779-8.

No 867 Erik Herzog: An Approach to Systems Engineering Tool Data Representation and Exchange, 2004, ISBN 91-7373929-4.

No 872 Aseel Berglund: Augmenting the Remote Control: Studies in Complex Information Navigation for Digital TV, 2004, ISBN 91-7373-940-5.

No 869 Jo Skåmedal: Telecommuting's Implications on Travel and Travel Patterns, 2004, ISBN 91-7373-935-9.

No 870 Linda Askenäs: The Roles of IT - Studies of Organising when Implementing and Using Enterprise Systems, 2004, ISBN 91-7373-936-7.

No 874 Annika Flycht-Eriksson: Design and Use of Ontologies in Information-Providing Dialogue Systems, 2004, ISBN 91-7373-947-2.
No 873 Peter Bunus: Debugging Techniques for Equation-Based Languages, 2004, ISBN 91-7373-941-3.

No 876 Jonas Mellin: Resource-Predictable and Efficient Monitoring of Events, 2004, ISBN 91-7373-956-1.

No 883 Magnus Bång: Computing at the Speed of Paper: Ubiquitous Computing Environments for Healthcare Professionals, 2004, ISBN 91-7373-971-5

No 882 Robert Eklund: Disfluency in Swedish human-human and human-machine travel booking dialogues, 2004, ISBN 91-7373-966-9.

No 887 Anders Lindström: English and other Foreign Linguistic Elements in Spoken Swedish. Studies of Productive Processes and their Modelling using Finite-State Tools, 2004, ISBN 91-7373-981-2.

No 889 Zhiping Wang: Capacity-Constrained Production-inventory systems - Modelling and Analysis in both a traditional and an e-business context, 2004, ISBN 9185295-08-6.

No 893 Pernilla Qvarfordt: Eyes on Multimodal Interaction, 2004, ISBN 91-85295-30-2.

No 910 Magnus Kald: In the Borderland between Strategy and Management Control - Theoretical Framework and Empirical Evidence, 2004, ISBN 91-85295-82-5.

No 918 Jonas Lundberg: Shaping Electronic News: Genre Perspectives on Interaction Design, 2004, ISBN 91-8529714-3.

No 900 Mattias Arvola: Shades of use: The dynamics of interaction design for sociable use, 2004, ISBN 91-8529542-6.

No 920 Luis Alejandro Cortés: Verification and Scheduling Techniques for Real-Time Embedded Systems, 2004, ISBN 91-85297-21-6.

No 929 Diana Szentivanyi: Performance Studies of FaultTolerant Middleware, 2005, ISBN 91-85297-58-5.

No 933 Mikael Cäker: Management Accounting as Constructing and Opposing Customer Focus: Three Case Studies on Management Accounting and Customer Relations, 2005, ISBN 91-85297-64-X.

No 937 Jonas Kvarnström: TALplanner and Other Extensions to Temporal Action Logic, 2005, ISBN 91-85297-75-5.

No 938 Bourhane Kadmiry: Fuzzy Gain-Scheduled Visual Servoing for Unmanned Helicopter, 2005, ISBN 9185297-76-3.

No 945 Gert Jervan: Hybrid Built-In Self-Test and Test Generation Techniques for Digital Systems, 2005, ISBN: 91-85297-97-6.

No 946 Anders Arpteg: Intelligent Semi-Structured Information Extraction, 2005, ISBN 91-85297-98-4.

No 947 Ola Angelsmark: Constructing Algorithms for Constraint Satisfaction and Related Problems - Methods and Applications, 2005, ISBN 91-85297-99-2.

No 963 Calin Curescu: Utility-based Optimisation of Resource Allocation for Wireless Networks, 2005, ISBN 91-8545707-8.

No 972 Björn Johansson: Joint Control in Dynamic Situations, 2005, ISBN 91-85457-31-0.

No 974 Dan Lawesson: An Approach to Diagnosability Analysis for Interacting Finite State Systems, 2005, ISBN 9185457-39-6.

No 979 Claudiu Duma: Security and Trust Mechanisms for Groups in Distributed Services, 2005, ISBN 91-85457-54$\mathrm{X}$.

No 983 Sorin Manolache: Analysis and Optimisation of RealTime Systems with Stochastic Behaviour, 2005, ISBN 9185457-60-4.

No 986 Yuxiao Zhao: Standards-Based Application Integration for Business-to-Business Communications, 2005, ISBN 91-85457-66-3.

No 1004 Patrik Haslum: Admissible Heuristics for Automated Planning, 2006, ISBN 91-85497-28-2. 
No 1005 Aleksandra Tešanovic: Developing Reusable and Reconfigurable Real-Time Software using Aspects and Components, 2006, ISBN 91-85497-29-0.

No 1008 David Dinka: Role, Identity and Work: Extending the design and development agenda, 2006, ISBN 91-8549742-8.

No 1009 Iakov Nakhimovski: Contributions to the Modeling and Simulation of Mechanical Systems with Detailed Contact Analysis, 2006, ISBN 91-85497-43-X.

No 1013 Wilhelm Dahllöf: Exact Algorithms for Exact Satisfiability Problems, 2006, ISBN 91-85523-97-6.

No 1016 Levon Saldamli: PDEModelica - A High-Level Language for Modeling with Partial Differential Equations, 2006, ISBN 91-85523-84-4.

No 1017 Daniel Karlsson: Verification of Component-based Embedded System Designs, 2006, ISBN 91-85523-79-8

No 1018 Ioan Chisalita: Communication and Networking Techniques for Traffic Safety Systems, 2006, ISBN 9185523-77-1.

No 1019 Tarja Susi: The Puzzle of Social Activity - The Significance of Tools in Cognition and Cooperation, 2006, ISBN 91-85523-71-2.

No 1021 Andrzej Bednarski: Integrated Optimal Code Generation for Digital Signal Processors, 2006, ISBN 91-85523-69-0.

No 1022 Peter Aronsson: Automatic Parallelization of EquationBased Simulation Programs, 2006, ISBN 91-85523-68-2.

No 1030 Robert Nilsson: A Mutation-based Framework for Automated Testing of Timeliness, 2006, ISBN 91-8552335-6.

No 1034 Jon Edvardsson: Techniques for Automatic Generation of Tests from Programs and Specifications, 2006, ISBN 91-85523-31-3.

No 1035 Vaida Jakoniene: Integration of Biological Data, 2006, ISBN 91-85523-28-3.

No 1045 Genevieve Gorrell: Generalized Hebbian Algorithms for Dimensionality Reduction in Natural Language Processing, 2006, ISBN 91-85643-88-2.

No 1051 Yu-Hsing Huang: Having a New Pair of Glasses Applying Systemic Accident Models on Road Safety, 2006, ISBN 91-85643-64-5.

No 1054 Åsa Hedenskog: Perceive those things which cannot be seen - A Cognitive Systems Engineering perspective on requirements management, 2006, ISBN 91-85643-57-2.

No 1061 Cécile Åberg: An Evaluation Platform for Semantic Web Technology, 2007, ISBN 91-85643-31-9.

No 1073 Mats Grindal: Handling Combinatorial Explosion in Software Testing, 2007, ISBN 978-91-85715-74-9.

No 1075 Almut Herzog: Usable Security Policies for Runtime Environments, 2007, ISBN 978-91-85715-65-7.

No 1079 Magnus Wahlström: Algorithms, measures, and upper bounds for Satisfiability and related problems, 2007, ISBN 978-91-85715-55-8.

No 1083 Jesper Andersson: Dynamic Software Architectures, 2007, ISBN 978-91-85715-46-6.

No 1086 Ulf Johansson: Obtaining Accurate and Compre-hensible Data Mining Models - An Evolutionary Approach, 2007, ISBN 978-91-85715-34-3.

No 1089 Traian Pop: Analysis and Optimisation of Distributed Embedded Systems with Heterogeneous Scheduling Policies, 2007, ISBN 978-91-85715-27-5.

No 1091 Gustav Nordh: Complexity Dichotomies for CSP-related Problems, 2007, ISBN 978-91-85715-20-6.

No 1106 Per Ola Kristensson: Discrete and Continuous Shape Writing for Text Entry and Control, 2007, ISBN 978-9185831-77-7.

No 1110 He Tan: Aligning Biomedical Ontologies, 2007, ISBN 978-91-85831-56-2.

No 1112 Jessica Lindblom: Minding the body - Interacting socially through embodied action, 2007, ISBN 978-9185831-48-7.
No 1113 Pontus Wärnestål: Dialogue Behavior Management in Conversational Recommender Systems, 2007, ISBN 97891-85831-47-0.

No 1120 Thomas Gustafsson: Management of Real-Time Data Consistency and Transient Overloads in Embedded Systems, 2007, ISBN 978-91-85831-33-3.

No 1127 Alexandru Andrei: Energy Efficient and Predictable Design of Real-time Embedded Systems, 2007, ISBN 97891-85831-06-7.

No 1139 Per Wikberg: Eliciting Knowledge from Experts in Modeling of Complex Systems: Managing Variation and Interactions, 2007, ISBN 978-91-85895-66-3.

No 1143 Mehdi Amirijoo: QoS Control of Real-Time Data Services under Uncertain Workload, 2007, ISBN 978-9185895-49-6.

No 1150 Sanny Syberfeldt: Optimistic Replication with Forward Conflict Resolution in Distributed Real-Time Databases, 2007, ISBN 978-91-85895-27-4.

No 1155 Beatrice Alenljung: Envisioning a Future Decision Support System for Requirements Engineering - A Holistic and Human-centred Perspective, 2008, ISBN 97891-85895-11-3.

No 1156 Artur Wilk: Types for XML with Application to Xcerpt, 2008, ISBN 978-91-85895-08-3.

No 1183 Adrian Pop: Integrated Model-Driven Development Environments for Equation-Based Object-Oriented Languages, 2008, ISBN 978-91-7393-895-2.

No 1185 Jörgen Skågeby: Gifting Technologies - Ethnographic Studies of End-users and Social Media Sharing, 2008, ISBN 978-91-7393-892-1.

No 1187 Imad-Eldin Ali Abugessaisa: Analytical tools and information-sharing methods supporting road safety organizations, 2008, ISBN 978-91-7393-887-7.

No 1204 H. Joe Steinhauer: A Representation Scheme for Description and Reconstruction of Object Configurations Based on Qualitative Relations, 2008, ISBN 978-91-7393823-5.

No 1222 Anders Larsson: Test Optimization for Core-based System-on-Chip, 2008, ISBN 978-91-7393-768-9.

No 1238 Andreas Borg: Processes and Models for Capacity Requirements in Telecommunication Systems, 2009, ISBN 978-91-7393-700-9.

No 1240 Fredrik Heintz: DyKnow: A Stream-Based Know-ledge Processing Middleware Framework, 2009, ISBN 978-917393-696-5.

No 1241 Birgitta Lindström: Testability of Dynamic Real-Time Systems, 2009, ISBN 978-91-7393-695-8.

No 1244 Eva Blomqvist: Semi-automatic Ontology Construction based on Patterns, 2009, ISBN 978-91-7393-683-5.

No 1249 Rogier Woltjer: Functional Modeling of Constraint Management in Aviation Safety and Command and Control, 2009, ISBN 978-91-7393-659-0.

No 1260 Gianpaolo Conte: Vision-Based Localization and Guidance for Unmanned Aerial Vehicles, 2009, ISBN 978-91-7393-603-3.

No 1262 AnnMarie Ericsson: Enabling Tool Support for Formal Analysis of ECA Rules, 2009, ISBN 978-91-7393-598-2.

No 1266 Jiri Trnka: Exploring Tactical Command and Control: A Role-Playing Simulation Approach, 2009, ISBN 978-917393-571-5.

No 1268 Bahlol Rahimi: Supporting Collaborative Work through ICT - How End-users Think of and Adopt Integrated Health Information Systems, 2009, ISBN 978-91-7393550-0.

No 1274 Fredrik Kuivinen: Algorithms and Hardness Results for Some Valued CSPs, 2009, ISBN 978-91-7393-525-8.

No 1281 Gunnar Mathiason: Virtual Full Replication for Scalable Distributed Real-Time Databases, 2009, ISBN 978-917393-503-6. 
No 1290 Viacheslav Izosimov: Scheduling and Optimization of Fault-Tolerant Distributed Embedded Systems, 2009, ISBN 978-91-7393-482-4.

No 1294 Johan Thapper: Aspects of a Constraint Optimisation Problem, 2010, ISBN 978-91-7393-464-0.

No 1306 Susanna Nilsson: Augmentation in the Wild: User Centered Development and Evaluation of Augmented Reality Applications, 2010, ISBN 978-91-7393-416-9.

No 1313 Christer Thörn: On the Quality of Feature Models, 2010, ISBN 978-91-7393-394-0.

No 1321 Zhiyuan He: Temperature Aware and Defect-Probability Driven Test Scheduling for System-on-Chip, 2010, ISBN 978-91-7393-378-0.

No 1333 David Broman: Meta-Languages and Semantics for Equation-Based Modeling and Simulation, 2010, ISBN 978-91-7393-335-3.

No 1337 Alexander Siemers: Contributions to Modelling and Visualisation of Multibody Systems Simulations with Detailed Contact Analysis, 2010, ISBN 978-91-7393-3179.

No 1354 Mikael Asplund: Disconnected Discoveries: Availability Studies in Partitioned Networks, 2010, ISBN 978-917393-278-3.

No 1359 Jana Rambusch: Mind Games Extended: Understanding Gameplay as Situated Activity, 2010, ISBN 978-91-7393252-3.

No 1373 Sonia Sangari: Head Movement Correlates to Focus Assignment in Swedish,2011,ISBN 978-91-7393-154-0.

No 1374 Jan-Erik Källhammer: Using False Alarms when Developing Automotive Active Safety Systems, 2011, ISBN 978-91-7393-153-3.

No 1375 Mattias Eriksson: Integrated Code Generation, 2011, ISBN 978-91-7393-147-2.

No 1381 Ola Leifler: Affordances and Constraints of Intelligent Decision Support for Military Command and Control Three Case Studies of Support Systems, 2011, ISBN 97891-7393-133-5.

No 1386 Soheil Samii: Quality-Driven Synthesis and Optimization of Embedded Control Systems, 2011, ISBN 978-91-7393102-1.

No 1419 Erik Kuiper: Geographic Routing in Intermittentlyconnected Mobile Ad Hoc Networks: Algorithms and Performance Models, 2012, ISBN 978-91-7519-981-8.

No 1451 Sara Stymne: Text Harmonization Strategies for PhraseBased Statistical Machine Translation, 2012, ISBN 97891-7519-887-3.

No 1455 Alberto Montebelli: Modeling the Role of Energy Management in Embodied Cognition, 2012, ISBN 978-917519-882-8.

No 1465 Mohammad Saifullah: Biologically-Based Interactive Neural Network Models for Visual Attention and Object Recognition, 2012, ISBN 978-91-7519-838-5.

No 1490 Tomas Bengtsson: Testing and Logic Optimization Techniques for Systems on Chip, 2012, ISBN 978-917519-742-5.

No 1481 David Byers: Improving Software Security by Preventing Known Vulnerabilities, 2012, ISBN 978-91-7519-784-5.

No 1496 Tommy Färnqvist: Exploiting Structure in CSP-related Problems, 2013, ISBN 978-91-7519-711-1.

No 1503 John Wilander: Contributions to Specification, Implementation, and Execution of Secure Software, 2013, ISBN 978-91-7519-681-7.

No 1506 Magnus Ingmarsson: Creating and Enabling the Useful Service Discovery Experience, 2013, ISBN 978-91-7519662-6.

No 1547 Wladimir Schamai: Model-Based Verification of Dynamic System Behavior against Requirements: Method, Language, and Tool, 2013, ISBN 978-91-7519-505-6.

No 1551 Henrik Svensson: Simulations, 2013, ISBN 978-91-7519491-2.
No 1559 Sergiu Rafiliu: Stability of Adaptive Distributed RealTime Systems with Dynamic Resource Management, 2013, ISBN 978-91-7519-471-4.

No 1581 Usman Dastgeer: Performance-aware Component Composition for GPU-based Systems, 2014, ISBN 97891-7519-383-0.

No 1602 Cai Li: Reinforcement Learning of Locomotion based on Central Pattern Generators, 2014, ISBN 978-91-7519-3137.

No 1652 Roland Samlaus: An Integrated Development Environment with Enhanced Domain-Specific Interactive Model Validation, 2015, ISBN 978-91-7519-090-7.

No 1663 Hannes Uppman: On Some Combinatorial Optimization Problems: Algorithms and Complexity, 2015, ISBN 97891-7519-072-3.

No 1664 Martin Sjölund: Tools and Methods for Analysis, Debugging, and Performance Improvement of EquationBased Models, 2015, ISBN 978-91-7519-071-6.

No 1666 Kristian Stavåker: Contributions to Simulation of Modelica Models on Data-Parallel Multi-Core Architectures, 2015, ISBN 978-91-7519-068-6.

No 1680 Adrian Lifa: Hardware/Software Codesign of Embedded Systems with Reconfigurable and Heterogeneous Platforms, 2015, ISBN 978-91-7519-040-2.

No 1685 Bogdan Tanasa: Timing Analysis of Distributed Embedded Systems with Stochastic Workload and Reliability Constraints, 2015, ISBN 978-91-7519-022-8.

No 1691 Håkan Warnquist: Troubleshooting Trucks - Automated Planning and Diagnosis, 2015, ISBN 978-91-7685-993-3.

No 1702 Nima Aghaee: Thermal Issues in Testing of Advanced Systems on Chip, 2015, ISBN 978-91-7685-949-0.

No 1715 Maria Vasilevskaya: Security in Embedded Systems: A Model-Based Approach with Risk Metrics, 2015, ISBN 978-91-7685-917-9.

No 1733 Victor Lagerkvist: Strong Partial Clones and the Complexity of Constraint Satisfaction Problems: Limitations and Applications, 2016, ISBN 978-91-7685856-1.

\section{Linköping Studies in Arts and Science}

No 504 Ing-Marie Jonsson: Social and Emotional Characteristics of Speech-based In-Vehicle Information Systems: Impact on Attitude and Driving Behaviour, 2009, ISBN 978-917393-478-7.

No 586 Fabian Segelström: Stakeholder Engagement for Service Design: How service designers identify and communicate insights, 2013, ISBN 978-91-7519-554-4.

No 618 Johan Blomkvist: Representing Future Situations of Service: Prototyping in Service Design, 2014, ISBN 97891-7519-343-4.

No 620 Marcus Mast: Human-Robot Interaction for SemiAutonomous Assistive Robots, 2014, ISBN 978-91-7519319-9.

\section{Linköping Studies in Statistics}

No 9 Davood Shahsavani: Computer Experiments Designed to Explore and Approximate Complex Deterministic Models, 2008, ISBN 978-91-7393-976-8.

No 10 Karl Wahlin: Roadmap for Trend Detection and Assessment of Data Quality, 2008, ISBN 978-91-7393-7924.

No 11 Oleg Sysoev: Monotonic regression for large multivariate datasets, 2010, ISBN 978-91-7393-412-1.

No 13 Agné Burauskaite-Harju: Characterizing Temporal Change and Inter-Site Correlations in Daily and Sub-daily Precipitation Extremes, 2011, ISBN 978-91-7393-110-6. 
No 1 Karin Axelsson: Metodisk systemstrukturering- att skapa samstämmighet mellan informationssystem-arkitektur och verksamhet, 1998. ISBN-9172-19-296-8.

No 2 Stefan Cronholm: Metodverktyg och användbarhet - en studie av datorstödd metodbaserad systemutveckling, 1998, ISBN-9172-19-299-2.

No 3 Anders Avdic: Användare och utvecklare - om anveckling med kalkylprogram, 1999. ISBN-91-7219-6068.

No 4 Owen Eriksson: Kommunikationskvalitet hos informationssystem och affärsprocesser, 2000, ISBN 91-7219811-7.

No 5 Mikael Lind: Från system till process - kriterier för processbestämning vid verksamhetsanalys, 2001, ISBN 91-7373-067-X.

No 6 Ulf Melin: Koordination och informationssystem i företag och nätverk, 2002, ISBN 91-7373-278-8.

No 7 Pär J. Ågerfalk: Information Systems Actability - Understanding Information Technology as a Tool for Business Action and Communication, 2003, ISBN 917373-628-7.

No 8 Ulf Seigerroth: Att förstå och förändra systemutvecklingsverksamheter - en taxonomi för metautveckling, 2003, ISBN91-7373-736-4.

No 9 Karin Hedström: Spår av datoriseringens värden Effekter av IT i äldreomsorg, 2004, ISBN 91-7373-963-4.

No 10 Ewa Braf: Knowledge Demanded for Action - Studies on Knowledge Mediation in Organisations, 2004, ISBN 9185295-47-7.

No 11 Fredrik Karlsson: Method Configuration method and computerized tool support, 2005, ISBN 91-85297-48-8.

No 12 Malin Nordström: Styrbar systemförvaltning - Att organisera systemförvaltningsverksamhet med hjälp av effektiva förvaltningsobjekt, 2005, ISBN 91-85297-60-7.

No 13 Stefan Holgersson: Yrke: POLIS - Yrkeskunskap, motivation, IT-system och andra förutsättningar för polisarbete, 2005, ISBN 91-85299-43-X.

No 14 Benneth Christiansson, Marie-Therese Christiansson: Mötet mellan process och komponent - mot ett ramverk för en verksamhetsnära kravspecifikation vid anskaffning av komponent-baserade informationssystem, 2006, ISBN 91-85643-22-X. 